\title{
FILTRATION OF RADIOACTIVE AEROSOLS BY GLASS FIBERS
}

\author{
PART TWO. APPENDICES
}

BY

A. G. BLASEWITZ, R. V. CARLISLE, B. F. JUDSON, M. F. KATZER,

E. F. KURTZ, W. C. SCHMIDT AND B. WEIDENBAUM

TECHNICAL DIVISIONS

APRIL 16, 1951

HANF́ORD WORKS

RICHLAND, WASHINGTON

GENERAL GLECTRIC 


\section{DISCLAIMER}

This report was prepared as an account of work sponsored by an agency of the United States Government. Neither the United States Government nor any agency Thereof, nor any of their employees, makes any warranty, express or implied, or assumes any legal liability or responsibility for the accuracy, completeness, or usefulness of any information, apparatus, product, or process disclosed, or represents that its use would not infringe privately owned rights. Reference herein to any specific commercial product, process, or service by trade name, trademark, manufacturer, or otherwise does not necessarily constitute or imply its endorsement, recommendation, or favoring by the United States Government or any agency thereof. The views and opinions of authors expressed herein do not necessarily state or reflect those of the United States Government or any agency thereof. 


\section{DISCLAIMER}

Portions of this document may be illegible in electronic image products. Images are produced from the best available original document. 
This document consists of 120 pages. Copy No. of 249 copies. Series A

\section{FILTRATION OF RADIOACTIVE AEROSOLS BY GLASS FIBERS}

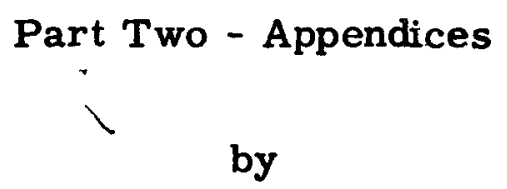

A. G. Blasewitz, R。V. Carlisle, B。F. Judson, M. F. Katzer, E. F. Kurtz, W. C. Schmidt and B. Weidenbaum Process Section Separations Technology Division

April 16, 1951

HANFORD WORKS

RICHLAND, WASHINGTON

Operated for the Atomic Energy Commission

by the

General Electric Company

under

Contract \# W-31-109-eng-52

\begin{tabular}{|c|c|c|c|c|c|}
\hline Route To & Read By & Date & Route To & Read By & Date \\
\hline & & & & & \\
\hline & & & & & \\
\hline & & & & & \\
\hline & & & & & \\
\hline & & & & & \\
\hline
\end{tabular}


1

2

3

4

5

6

7

8

9

10

11

12

13

14

15

16

17

18

19

20

21

22

23

24

25

$26-35$
A. B. Greninger - O. H. Greager

R. H. Beaton

H. M. Parker

C. C. Gamertsfelder - F. E. Adley

R. B. Richards

J. B. Work

J. E. Maider - R. S. Bell

V. R. Chapman - R. C. Grant

J. S. Parker - J. M. Frame

V. W. Wood - E. M. Johnston

F. W. Woodfield

O. F. Hill

V. R. Cooper

B. Weidenbaum

A. G. Blasewitz

R. V. Carlisle

B. F. Judson

M. F. Katzer

E. F. Kurtz

W. C. Schmidt

$E$ and $C$ File

700 File

300 File

Pink Copy

Yellow Copy

Extra Copies 
Project Distribution

\section{EXTERNAL DISTRIBUTION}

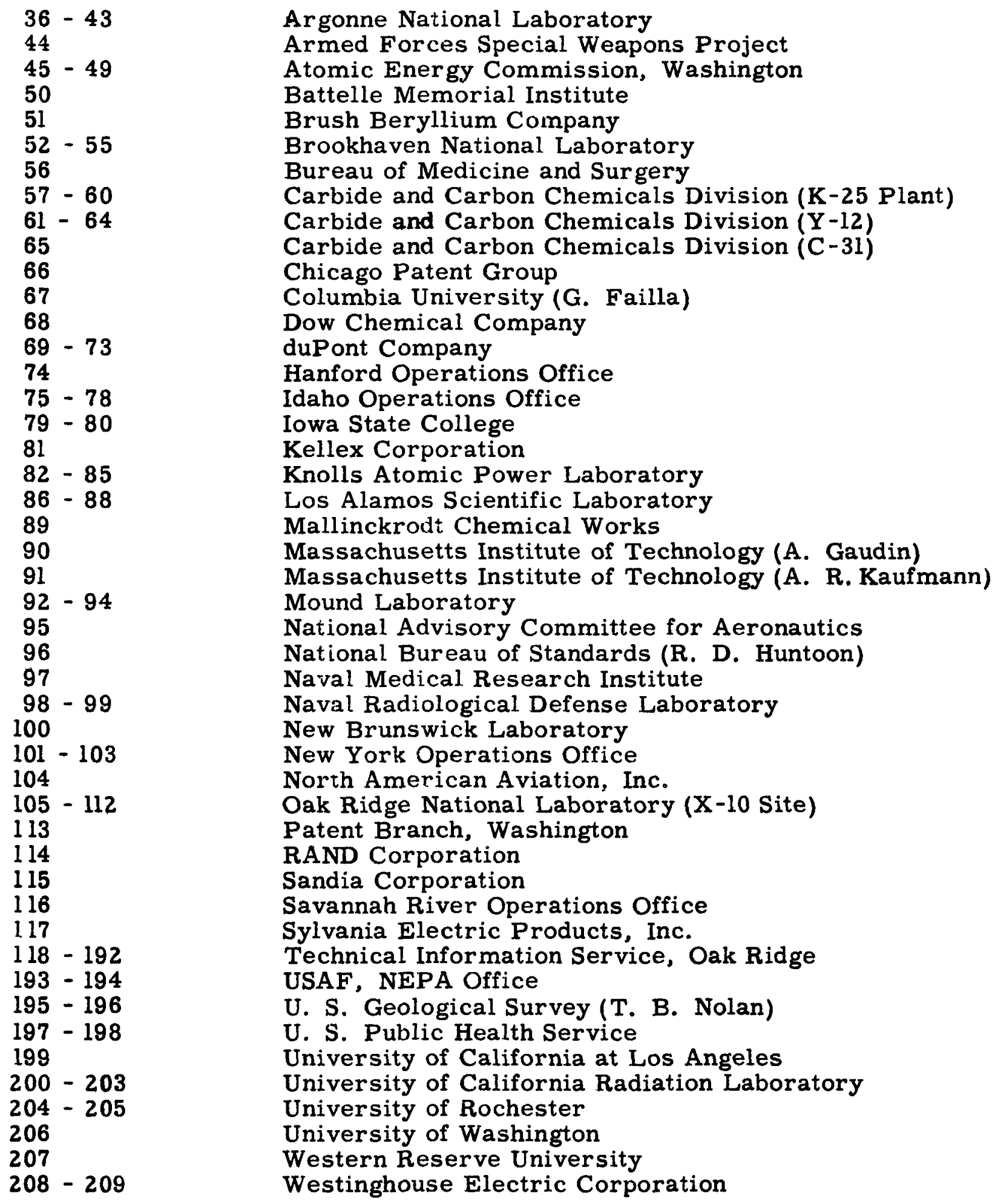

Argonne National Laboratory

Armed Forces Special Weapons Project

Atomic Energy Commission, Washington

Battelle Memorial Institute

Brush Beryllium Company

Brookhaven National Laboratory

Bureau of Medicine and Surgery

Carbide and Carbon Chemicals Division (K-25 Plant)

Carbide and Carbon Chemicals Division ( $\mathrm{Y}-12)$

Carbide and Carbon Chemicals Division (C-3I)

Chicago Patent Group

Columbia University (G. Failla)

Dow Chemical Company

duPont Company

Hanford Operations Office

Idaho Operations Office

Iowa State College

Kellex Corporation

Knolls Atomic Power Laboratory

Los Alamos Scientific Laboratory

Mallinckrodt Chemical Works

Massachusetts Institute of Technology (A. Gaudin)

Massachusetts Institute of Technology (A. R. Kaufmann)

Mound Laboratory

National Advisory Committee for Aeronautics

National Bureau of Standards (R. D. Huntoon)

Naval Medical Research Institute

Naval Radiological Defense Laboratory

New Brunswick Laboratory

New York Operations Office

North American Aviation, Inc.

Oak Ridge National Laboratory (X-10 Site)

Patent Branch, Washington

RAND Corporation

Sandia Corporation

Savannah River Operations Office

Sylvania Electric Products, Inc.

Technical Information Service, Oak Ridge

USAF, NEPA Office

U. S. Geological Survey (T. B. Nolan)

U. S. Public Health Service

University of California at Los Angeles

University of California Radiation Laboratory

University of Rochester

University of Washington

Western Reserve University

Westinghouse Electric Corporation 
Supplementary Distribution

COPY NUMBER

$210-213$
214
215
216
217
218
$219-220$
221
222
223
224
$225-226$
$227-236$
237
238
$239-243$
244

245

$246-248$

249

\section{EXTERNAL DISTRIBUTION}

Atomic Energy Project, Chalk River

California Institute of Technology Attn: R. F. Bacher

Chief of Naval Research

H. K. Ferguson Company

Harshaw Chemical Corporation

Isotopes Division (Mr. McCormick)

Library of Congress, Acquisition Department (J. W. Cormn)

National Bureau of Standards (Library)

National Research Council, Ottawa

Naval Research Laboratory

Nucleonics

Oak Ridge Institute of Nuclear Studies

United Kingdom Scientific Mission (M. Greenhill)

USAF, Eglin Air Force.Base (Technical Library)

USAF, Wright-Patterson Air Force Base (Rodney Nudenberg)

USAF, Wright-Patterson Air Force Base (CADO)

U. S. Army, Army Medical Service Graduate School (Col. W. S. Stone)

U. S. Army, Atomic Energy Branch (Lt. Col. A. W. Betts)

U. S. Army, Technical Command ( Col。 J. H. Rothschild, Attn: Technical Library)

UT-AEC Agricultural Research Program (Charles S. Hobbs) 


\section{TABLE OF CONTENTS}

Page No.

INTRODUCTION.

6

APPENDIX A - Modified Cascade Impactor.............. $17-27$

APPENDIX B - Permeability Tests................. $28-48$

APPENDIX C - Collection Efficiency Tests............. $49-73$

APPENDIX D - Nomographs.................... $74-79$

APPENDIX E - Life Expectancy Tests................ $80-120$ 


\section{INTRODUCTION}

For purposes of convenience, the report entitled "The Filtration of Radioactive Aerosols By Glass Fibers" has been published in two volumes. Part one (HW-20847, 4-16-51) contains the text of the report and describes the development program which led to the design of highly efficient, glass fiber filters. Part two (HW-20847, 4-16-51) is composed of five appendices each of which pertains to a separate phase of the program. Four of the appendices contain experimental data presented in graphical form. The remaining appendix consists of a series of nomographs summarizing the permeability and efficiency characteristics of three types of glass fibers. 


\section{TABLE OF ILLUSTRATIONS}

\section{APPENDIX A}

Figure No.

Page No.

A-1

Sample Calculation of the Particle Size Distribution Determined by the Modified Cascade Impactor.......

A-2 - A-7 Logarithmic-Probability Graphs of Cumulative Per Cent of Activity Less than the Stage Median Value vs. the Particle Diameter for the Modified-CascadeImpactor Tests Performed on the Following Dates.

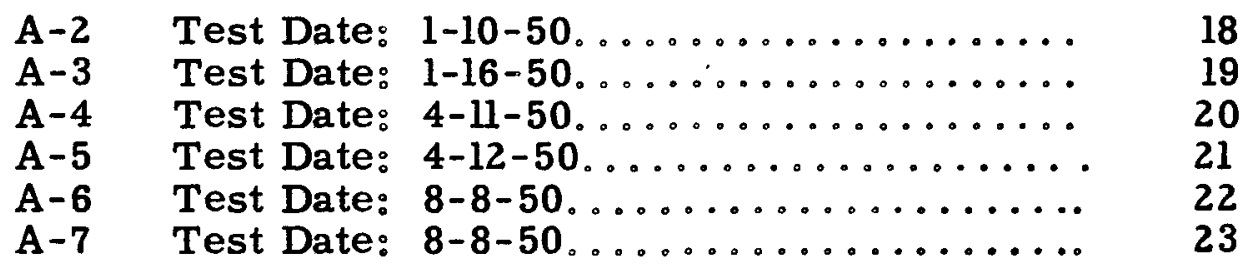

A-9 - A-10 Logarithmic-Probability Graphs of Cumulative Per Cent of Methylene Blue Less than the Stage Median Value vs. the Particle Diameter for the ModifiedCascade-Impactor Tests Performed on the Following Dates.

A-9 Test Date: $5-31-50 \ldots \ldots \ldots \ldots \ldots \ldots \ldots \ldots . \ldots . \ldots . \ldots . \ldots$

A-10 Test Date: 7-28-50.............. 26

A-11 An Arithmetic-Probability Graph of Figure A-9 ... 27 


\section{TABLE OF ILLUSTRATIONS}

\section{APPENDIX B}

Figure No.

Page No.

B-1 Graph of Pressure Drop vs. Superficial Velocity for No. 55 Fiberglas at 3.0 pcf.............. 28

B-2 Graph of Pressure Drop vs. Superficial Velocity for No. 55 Fiberglas at 6.0 pcf.............. 29

B-3 Graph of Pressure Drop vs. Superficial Velocity for No. 55 Fiberglas at 9.0 pcf...............

B-4 Cross-plot of Pressure Drop vs. Bed Depth for No. 55 Fiberglas. .....................

B-5 Cross-plot of Pressure Drop vs. Packing Density for No. 55 Fiberglas . . . . ................... 32

B-6 Graph of Pressure Drop vs. Superficial Velocity for No, 55P Fiberglas at 2.0 pcf. and 3.0 pcf....

B-7 Graph of Pressure Drop vs. Superficial Velocity

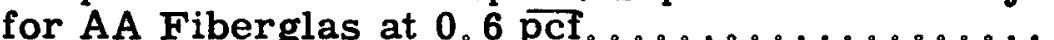

B-8 Graph of Pressure Drop vs. Superficial Velocity for AA Fiberglas at $1.2 \overline{p c f}_{0} \ldots \ldots \ldots \ldots \ldots$

B-9 Graph of Pressure Drop vs, Superficial Velocity for AA Fiberglas at 2,4 pcf. ..............

B-10 Cross-plot of Pressure Drop vs. Bed Depth

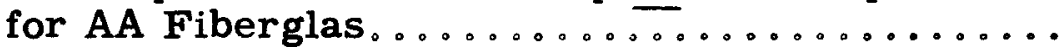

B-11 Cross-plot of Pressure Drop vs. Packing Density for AA Fiberglas . . . . . . . . . . . . . . . . . .

B-12 Graph of Pressure Drop vs. Superficial Velocity for B Fiberglas at 0.7 pcf and 1.4 pcf..........

B-13 Graph of Pressure Drop vs. Superficial Velocity for $B(L$ and $R$ ) Fiberglas at 3.5 pcf..........

B-14 Graph of Pressure Drop vs. Superficial Velocity for No. 450 Fiberglas at 5.7 pcf............ 


\section{APPENDIX B (Cont.)}

Figure No.

Page No.

B-15 Graph of Pressure Drop vs. Superficial Velocity for No. 600 Fiberglas at 5.1 pef.............. 42

B-16 Graph of Pressure Drop vs. Superficial Velocity for No. 800 Fiberglas at 6.6 pcf..............

B-17 Graph of Pressure Drop vs. Superficial Velocity

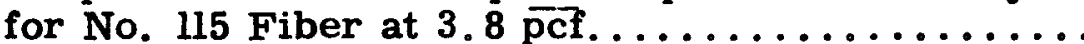

B-18 Graph of Pressure Drop vs. Superficial Velocity for No. $115 \mathrm{~K}$ Fiberglas at 1.5 pcf and 6.0 pcf.......

B-19 Graph of Pressure Drop vs. Superficial Velocity for No. 115K Fiberglas at 3.0 pcf. and 9.0 pcf......

B-20 Cross-plot of Pressure Drop vs. Bed Depth for

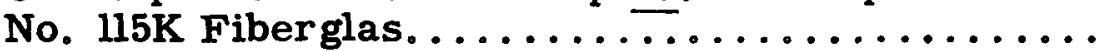

B-21 Cross-plot of Pressure Drop vs. Packing Density for No. ll5K Fiberglas. .................... 


\section{TABLE OF ILLUSTRATIONS}

\section{APPENDIX C}

Figure No.

Page No.

C-1 Graph of Collection Efficiency vs. Decontamina-

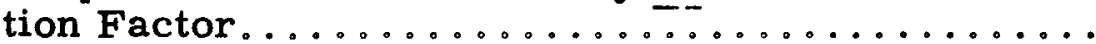

C-3 Graph of Decontamination Factor vs. Superficial Velocity for No. 55 Fiberglas at 4.5 pcf.........

C-4 Graph of Decontamination Factor vs. Superficial Velocity for No. 55 Fiberglas at 6.0 pcf.........

C-5 Graph of Decontamination Factor vs. Superficial Velocity for No. 55 Fiberglas at 9.0 pcf.........

C-6 Cross-plot of Decontamination Factor vs. Bed Depth for No. 55 Fiberglas...................

C-7 Cross-plot of Decontamination Factor vs. Packing Density for No. 55 Fiberglas...............

C-8 Graph of Decontamination Factor vs. Superficial Velocity for No. 55 P Fiberglas. .............

C-9 Graph of Decontamination Factor vs. Superficial Velocity for AA Fiberglas at 0.6 pcf..........

C-10 Graph of Decontamination Factor vs. Superficial Velocity for AA Fiberglas at 1.2 pcf. and $1.8 \mathrm{pcf} .$.

C-11 Cross-plot of Decontamination Factor vs. Bed Depth for AA Fiberglas. ................

C-12 Cross-plot of Decontamination Factor vs. Packing

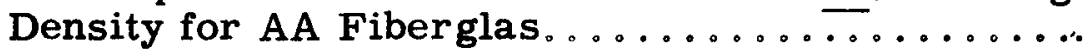

C-13 Graph of Decontamination Factor vs. Superficial Velocity for $B(L$ and $R$ ) Fiberglas........... 


\section{APPENDIX C (Cont.)}

Figure No.

Page No.

C-14 Graph of Decontamination Factor vs. Superficial

Velocity for B Fiberglas.................

C-15 Graph of Decontamination Factor vs. Superficial

Velocity for No. 450 Fiberglas.............

C-16 Graph of Decontamination Factor vs. Superficial

Velocity for No. 600 Fiberglas..............

C-17 Graph of Decontamination Factor vs. Superficial Velocity for No. 800 Fiberglas.............

C-18 Graph of Decontamination Factor vs. Superficial Velocity for No. 115 Fiber................

C-19 Cross-plot of Decontamination Factor vs. Bed Depth for No. 115 Fiber...................

C-20 Graph of Decontämination Factor vs. Superficial Velocity for No. 115K Fiberglas at 1.5 pcf........

C-21 Graph of Decontamination Factor vs. Superficial Velocity for No. 115K Fiberglas at 3.0 pcf.......

C-22 Graph of Decontamination Factor vs. Superficial Velocity for No. 115K Fiberglas at 6.0 pcf.......

C-23 Graph of Decontamination Factor vs. Superficial Velocity for No. 1l5K Fiberglas at 9.0 pcf.......

C-24 Cross-plot of Decontamination Factor vs. Bed Depth for No, $115 \mathrm{~K}$ Fiberglas................

C-25 Cross-plot of Decontamination Factor vs. Packing Density for No. $115 K$ Fiberglas. ............. 
APPENDIX D

Figure No.

Page No.

D-1 Nomograph of the Permeability Characteristics of No. 55 Fiberglas.................... 74

D-2 Nomograph of the Permeability Characteristics of AA Fiberglas...................... 75

D-3 Nomograph of the Permeability Characteristics of No. 115K Fiberglas.................. 76

D-4 Nomograph of the Efficiency Characteristics of No. 55 Fiberglas....................

D-5 Nomograph of the Efficiency Characteristics of AA Fiberglas......................

D-6 Nomograph of the Efficiency Characterisitcs of No. $115 K$ Fiberglas................... 
TABLE OF ILLUSTRATIONS

APPENDIX E

Figure No.

Page No.

E-1 - E-4 Graphs of Collection Efficiency and Pressure Drop vs. $\Sigma$ - Function for the Following Test Units Operated at 25 fpm.

E-1 Two Inches of No. 55 Fiberglas at

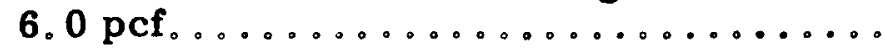

E-2 Four Inches of No. 55 Fiberglas at 3.0 pcf................... 81

E-3 Two Inches of No. 115K Fiberglas at 6.0 pcf.................... 82

E-4 Six Inches of No. 115K Fiberglas at

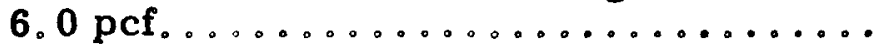

E-5 Graph of Pressure Drop vs. $\Sigma$ - Function for the First Sand Filter Test Unit ................

E-6 Graph of Pressure Drop vs. $\Sigma$ - Function for the Second Sand Filter Test Unit. .............

E-7 Graph of Pressure Drop vs $\quad \Sigma$ - Function for the Third Sand Filter Test Unit.............

E-8 Graph of the Radioactivity Distribution in a Plant

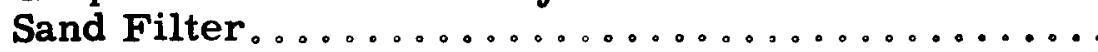

E-9 - E-14 Graphs of Pressure Drop vs. $\Sigma$ - Function for the Following Test Units Operated at $50 \mathrm{fpm}$.

E-9 Five Inches of No. 55 Fiberglas at 9.0 pcf. Protecting $1 / 4$-inch of AA Fiberglas at 1.2 pcf..............

E-10 Eleven Inches of No. 55 Fiberglas at 6.0 pcf. Protecting 1/4-inch of AA Fiberglas at 1.2 pcf $\ldots \ldots \ldots \ldots \ldots \ldots$

E-11 Five Inches of No. 55 Fiberglas at 3.0 pcf. Protecting 11 Inches of No. 55 Fiberglas at 6.0 pcf........... 


\section{APPENDIX E (Cont.)}

Figure No.

Page No.

E-12 Twelve Inches of No. 450 Fiberglas at 5.7 pcf. Protecting 5 Inches of No. 55 Fiberglas at 3.0 pcf.........

E-13 Twelve Inches of No。600 Fiberglas at 6.8 pcf. Protecting 12 Inches of No, 450 Fiberglas at 5.7 pcf . ......

E-14 Twelve Inches of No. 800 Fiberglas at 6.6 pcf. Protecting 12 Inches of No. 600 Fiberglas at 6.8 pcf........

E-15 - E-24 Graphs of Pressure Drop vs. $\Sigma$ - Function for the Following Test Units Operated at $25 \mathrm{fpm}$.

E-15 Eight Inches of No。 55 Fiberglas at 6. 0 pcf. Protecting $1 / 4$ Inch of AA Fiberglas at 1.2 pcf..............

E-16 Five Inches of No. 55 Fiberglas at 3.0 pcf. Protecting 8 Inches of

No. 55 Fiberglas at 6.0 pcf........

E-17 Five Inches of No. 115 Fiber at 3.8 pcf. Protecting 5 Inches of No. 55 Fiberglas at 3.0 pcf .........

E-18 Twelve Inches of No. 450 Fiberglas at 10 pcf. Protecting 5 Inches of No. 115 Fiber at 3.8 pcf. ............

E-19 Fourteen Inches of No. 55 Fiberglas at 1.5 pcf. Protecting 5 Inches of No. 55 Fiberglas at 3.0 pcf.........

E-20 Twelve Inches of No. 450 Fiberglas at 10 pcf. Protecting 14 Inches of No. 55 Fiberglas at 1.5 pcf.........

E-21 Twenty-four Inches of No. 450 Fiberglas at 5.0 pcf. Protecting 14 Inches of No.55 Fiberglas at 1.5 pcf...

E-22 Ten Inches of No. 55 Fiberglas at 1.5 pcf. Protecting 5 Inches of No. 55 Fiberglas at 3.0 pcf $\ldots \ldots \ldots \ldots \ldots \ldots$ 


\section{APPENDIX E (Cont.)}

Figure No.

Page No.

E-23 Twenty-four Inches of No. 450 Fiberglas at 2.0 pcf. Protecting 14 Inches of No. 55 Fiberglas at 1.5 pcf.........

E-24 Twenty-four Inches of No. 800 Fiberglas at 5.0 pcf. Protecting 14 Inches of No. 55 Fiberglas at 1.5 pcf.........

E-25 Graph of Pressure Drop vs. $\quad$ - Function for a Composite Test Unit Operated at $50 \mathrm{fpm} . . . \ldots \ldots$.

E-26A and Graphs of Pressure Drop vs. $\Sigma$ - Function for E-26B a Composite Test Unit Operated at $25 \mathrm{fpm}$

E-27 - E-29 Graphs of Pressure Drop vs. $\Sigma$ - Function for the Following Test Units Operated at $25 \mathrm{fpm}$.

E-27 Eight Inches of No. 55P Fiberglas at 6.0 pcf. Protecting $3 / 4$ Inch of AA Fiberglas at 1.2 pcf.

E-28 Six Inches of No. 55P Fiberglas at 3.0 pcf. Protecting 8 Inches of No. 55P Fiberglas at 6.0 pcf.

E-29 Eighteen Inches of No. 55P Fiberglas at 1.5 pcf. Protecting 8 Inches of No. 55P Fiberglas at 3.0 pcf.

E-30 - E-33 Graphs of Pressure Drop vs. $\Sigma$ - Function for the following Test Units Operated at $50 \mathrm{fpm}$.

E-30 Eight Inches of No. 115K Fiberglas at 9.0 pcf. Protecting $3 / 4$ Inch of AA Fiberglas at 1.2 pcf............. 110

E-31 Six Inches of No. 115K Fiberglas at 6.0 pcf. Protecting 8 Inches of No: $115 \mathrm{~K}$ Fiberglas at 9.0 pcf. . ..........

E-32 Six Inches of No. 115K Fiberglas at 3.0 pcf. Protecting 8 Inches of No. 115K Fiberglas at 6.0 pcf..........

E-33 Twelve Inches of No. 115K Fiberglas at 1.5 pcf. Protecting 6 Inches of No. 115K Fiberglas at 3.0 pcf......... 


\section{APPENDIX E (Cont.)}

Figure No.

Page No.

E-34 - E-40 Graphs of Pressure Drop vs. $\Sigma$ - Function for the Following Units Operated at $25 \mathrm{fpm}$.

(E-39; at $15 \mathrm{fpm}$ ).

E-34 Eight Inches of No. 115K Fiberglas at 9.0 pcf. Protecting $3 / 4$ Inch of AA Fiberglas at 1.2 pcf............. 114

E-35 Six Inches of No. $115 \mathrm{~K}$ Fiberglas at 6.0 pcf. Protecting 8 Inches of No. 115K Fiberglas at 9.0 pef.

E-36 Six Inches of No. 115K Fiberglas at

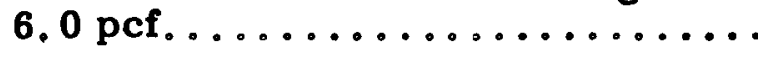

E-37 Six Inches of No. 115K Fiberglas at 3.0 pcf. Protecting 8 Inches of No. 1l5K Fiberglas at 6.0 pcf..........

E-38 Six Inches of No. 115K Fiberglas at 3.0 pcf. Protecting 8 Inches of No. 115K Fiberglas at 6.0 pcf. ............

E-39 Three Inches of No. 115K Fiberglas at 3.0 pcf. Protecting 6 Inches of No. $115 \mathrm{~K}$ Fiberglas at 6.0 pcf. (at 15 fpm.)

E-40 Twelve Inches of No. 115K Fiberglas at 1.5 pcf. Protecting 6 Inches of No. $115 \mathrm{~K}$ Fiberglas at 3.0 pcf. .......... 
Figure A-1

SAMPLE CALCULATION OF THE PARTICLE SIZE

DISTRIBUTION DETERMINED BY THE

MODIFIED CASCADE IMPACTOR

Test Date: 1-10-50

Sample Flow Rate: $0.5 \mathrm{cfm}$.

\begin{tabular}{|c|c|c|c|c|}
\hline Stage No. & $\begin{array}{c}\text { Stage Median } \\
\text { Diameter } \\
\text { Microns }\end{array}$ & $\begin{array}{c}\text { Radioactivity } \\
\text { Content } \\
\text { dpm. }\end{array}$ & $\begin{array}{c}\text { Stage } \\
\text { Median } \\
\text { Value } \\
\end{array}$ & $\begin{array}{c}\text { Cumulative } \\
\text { Per Cent of } \\
\text { Activity Less } \\
\text { Than the Stage } \\
\text { Median Value } \\
\end{array}$ \\
\hline I & 7.72 & 10,900 & 5,500 & 99.80 \\
\hline II & 3.24 & 30,700 & 15,400 & 99.02 \\
\hline III & 1.71 & 188,000 & 94,000 & 94.95 \\
\hline IV & 1.14 & 444,000 & 222,000 & 83.17 \\
\hline $\mathbf{v}$ & 0.68 & $2,010,000$ & $1,005,000$ & 37.45 \\
\hline
\end{tabular}




$$
18
$$

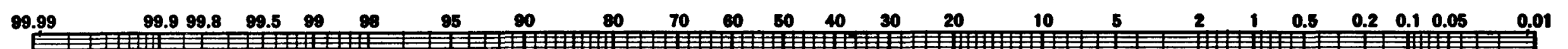

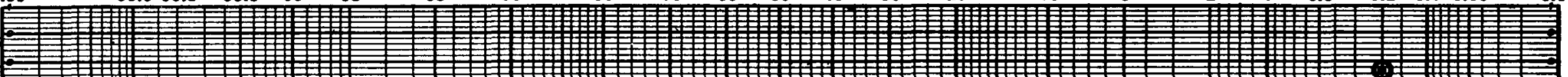
c

\section{Figure A-2}

PARTICLE SIZE DISTRIBUTION FOR.THE PROCESS-CELL AIR AS DETERMINED BY THE MODIFIED CASCADE MPACTOR

Test Date: $1-10-50$

Sample Flow Rate: $0.5 \mathrm{~cm}$. $+$ 进哣

IIC 靾 $\because+1$

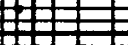
(1)

轧1 (4⿻肀二𠃋

$\stackrel{0}{0}$ 


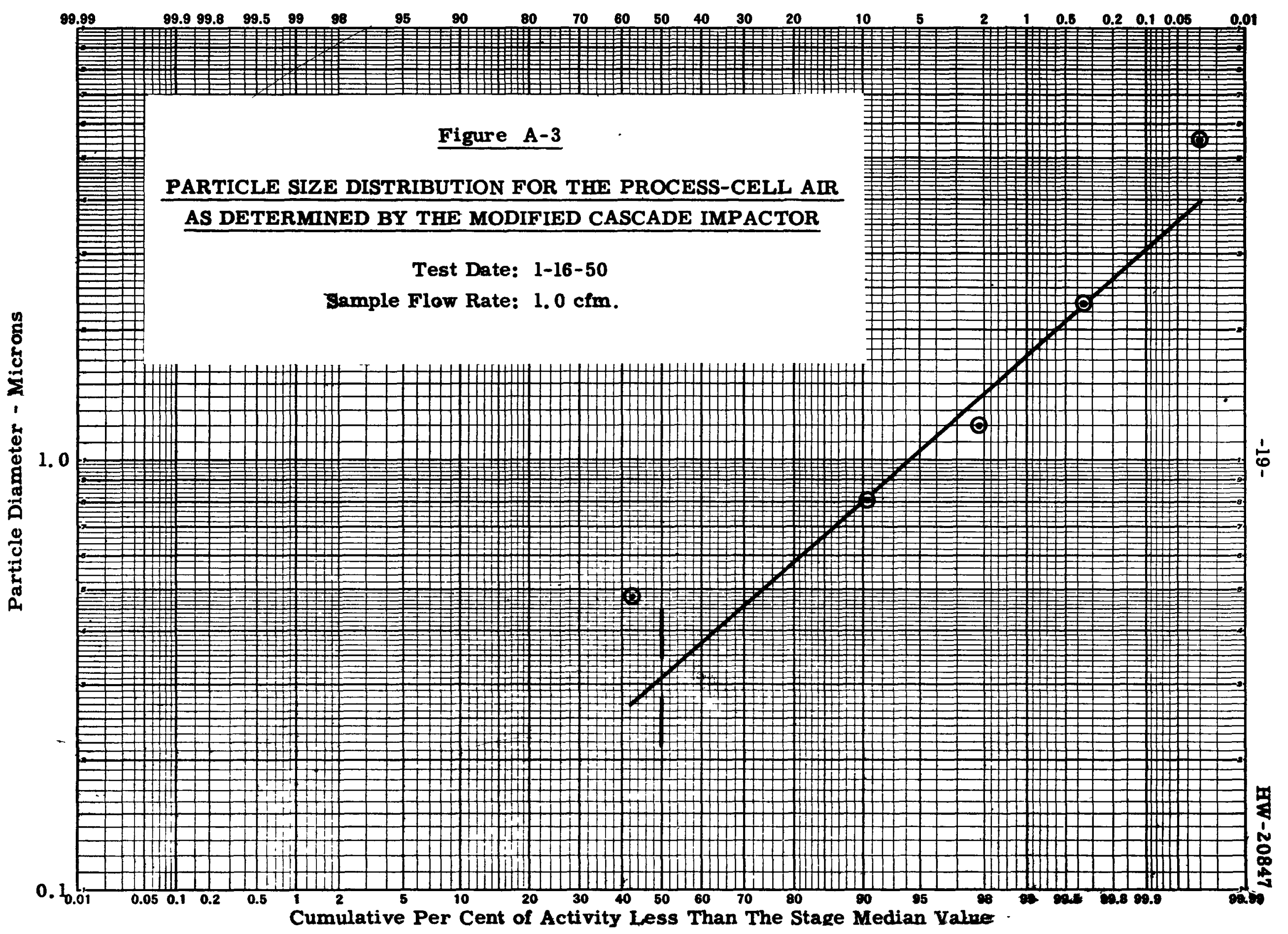


20

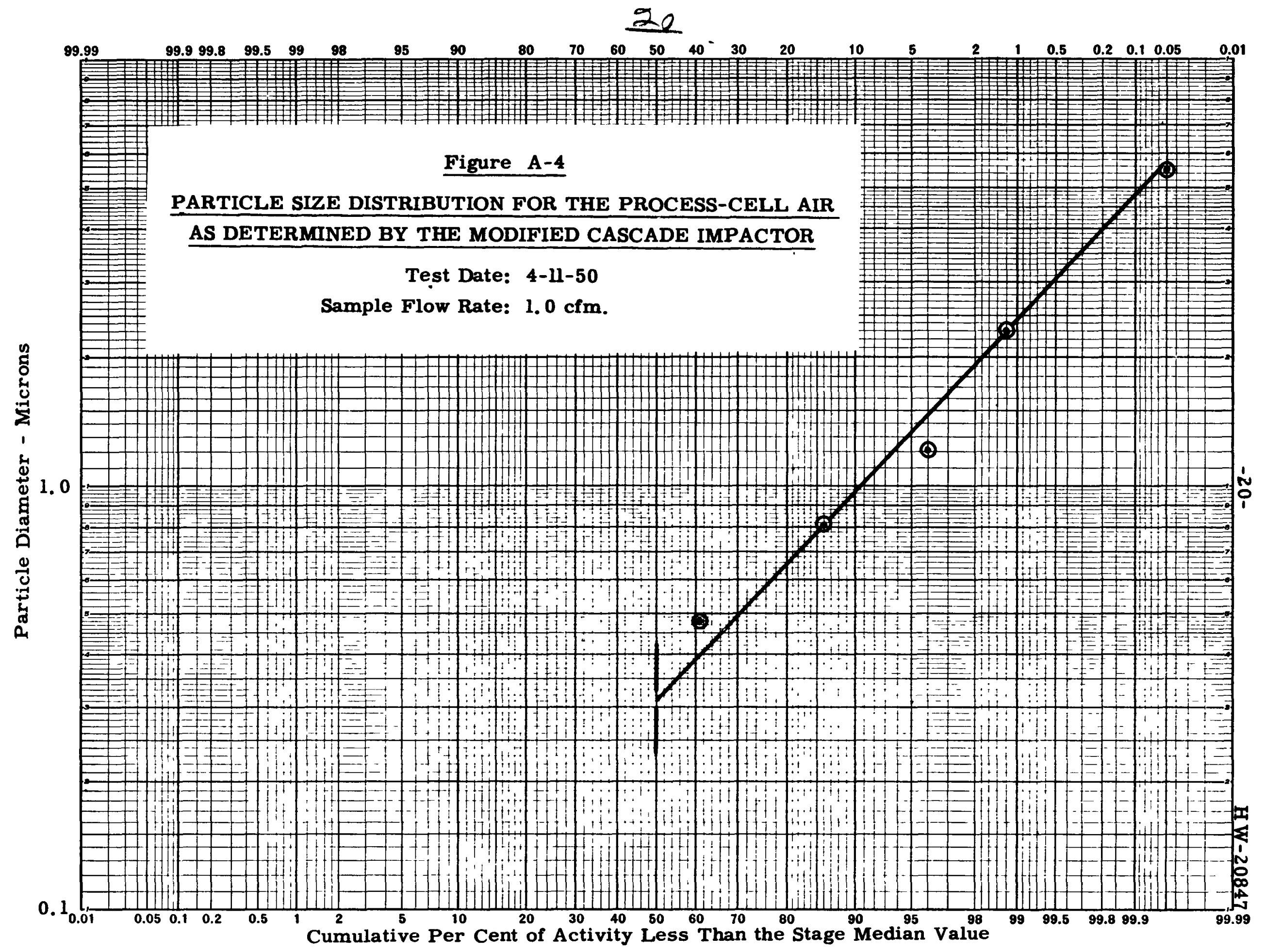




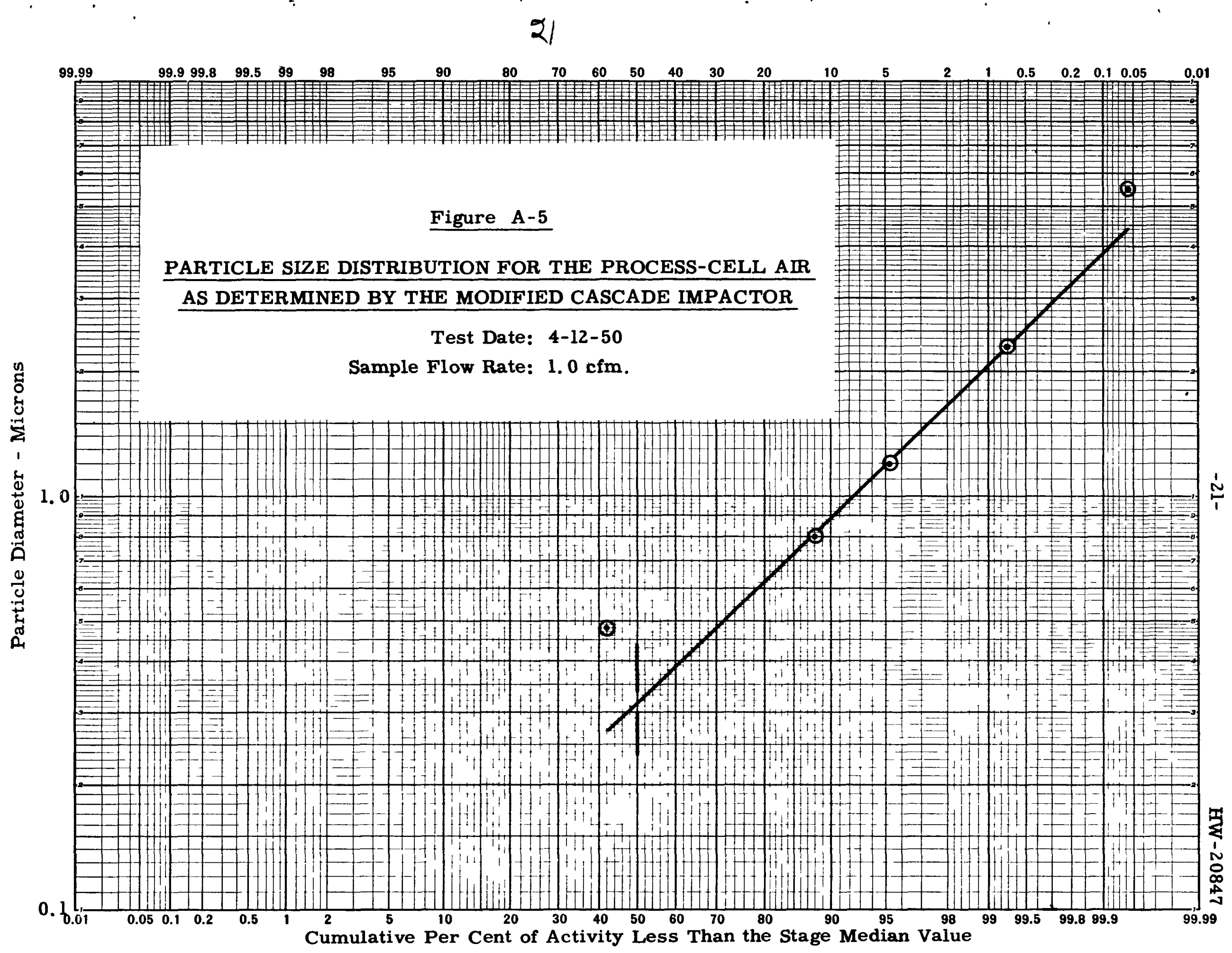


22

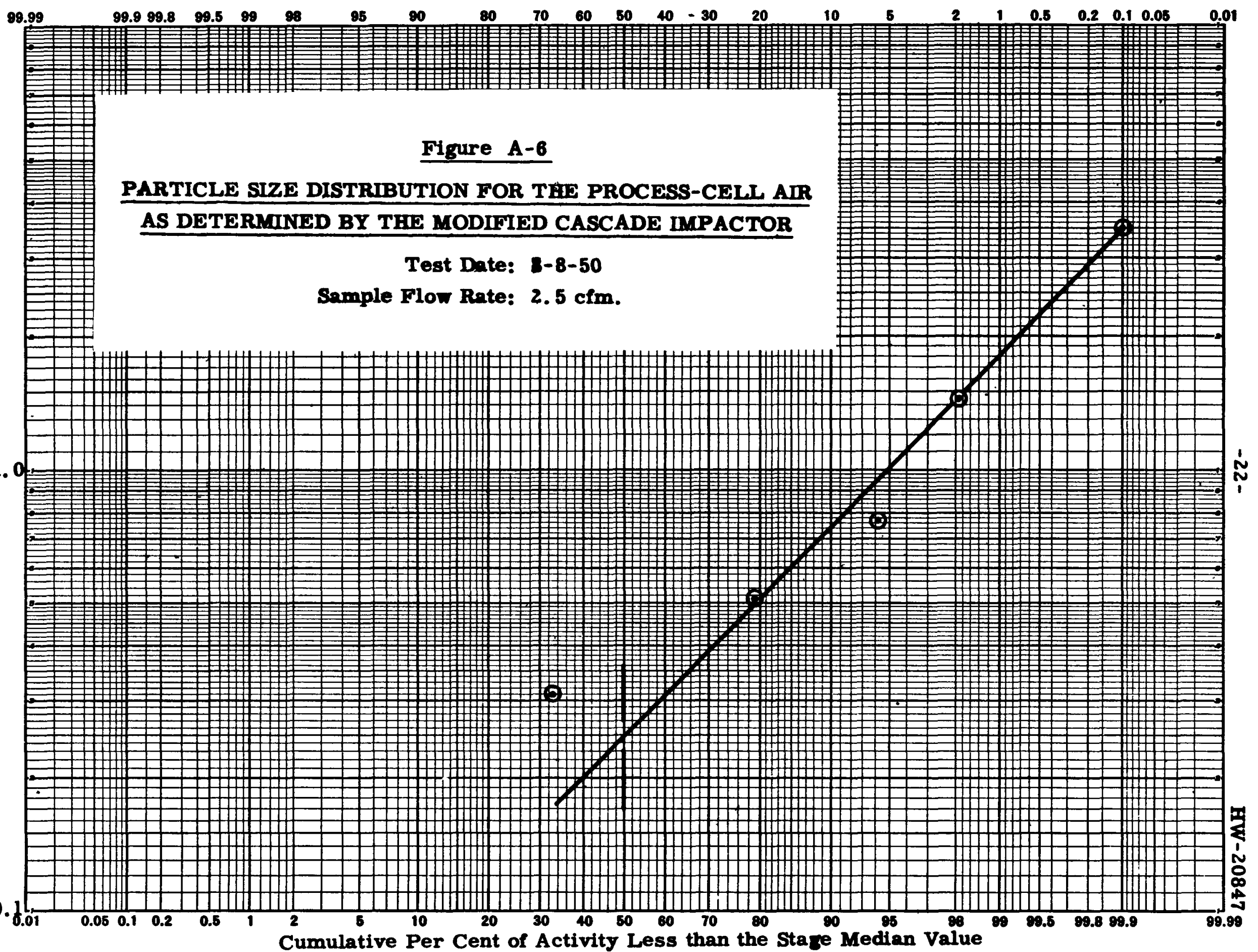


23

09

$\begin{array}{rrrrr}99.9 & 99.8 & 99.5 & 99 & 98\end{array}$

$95 \quad 90$

80

7060

$50 \quad 40 \quad 30$

20

10

5 2

$\begin{array}{llll}0.5 & 0.2 & 0.1 & 0.05\end{array}$ 0.01

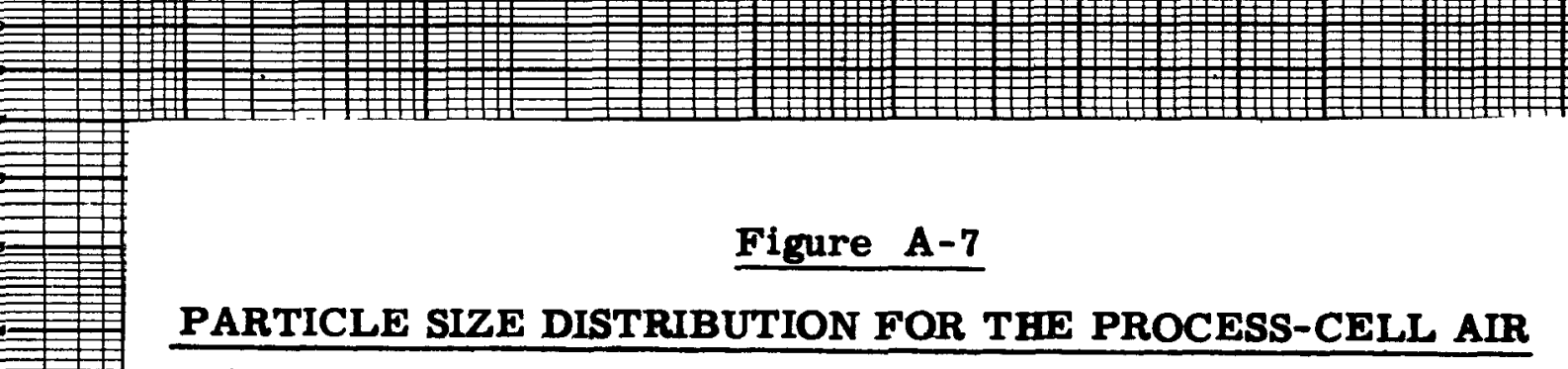
AS DETERMINED BY THE MODIFIED CASCADE IMPACTOR

Test Date: 8-8-50

Sample Flow Rate: $2.65 \mathrm{cfm}$.

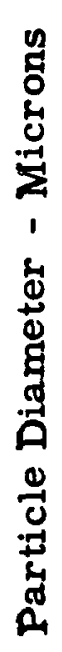

1.0

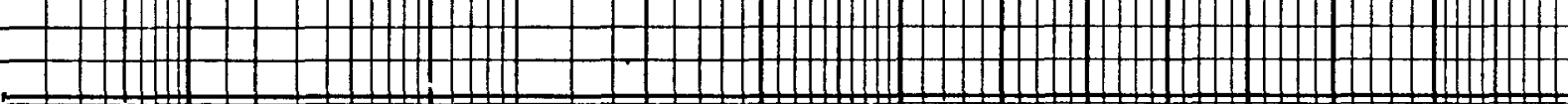

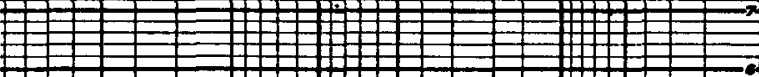

1 -

$12+2+210+2$

$\begin{array}{lll} & 1\end{array}$

\begin{tabular}{lll}
\hline 2 & 2
\end{tabular}

$12+2100$

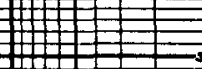

\begin{tabular}{l}
\hline 1213 \\
\hline
\end{tabular}

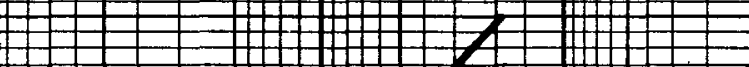
\#

\#

$1+$ 田

管

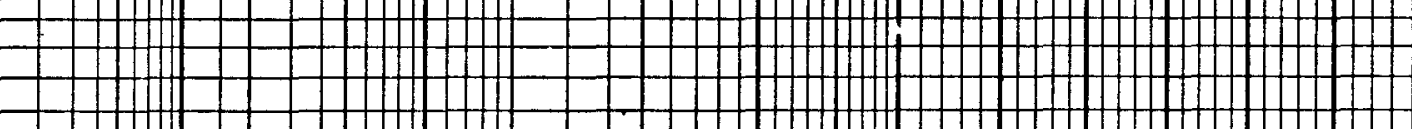

-1.

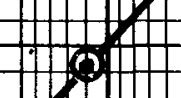

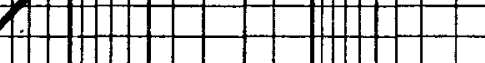

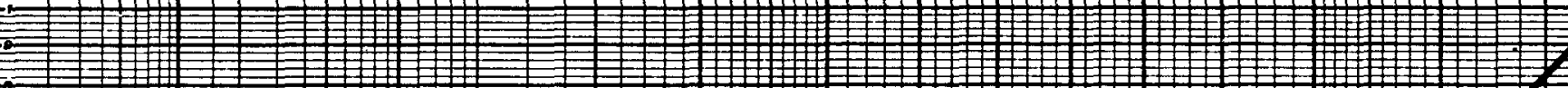

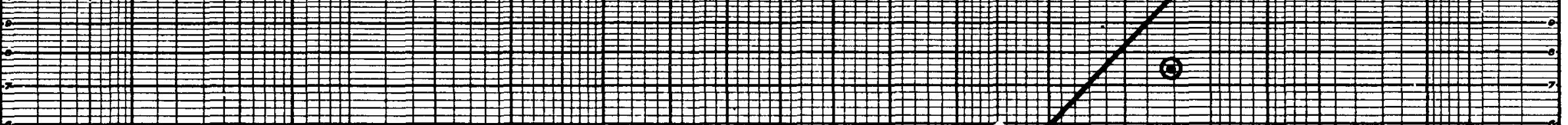

\section{: (2) +}

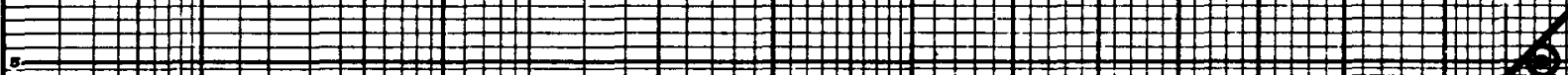

$1=12$ 2

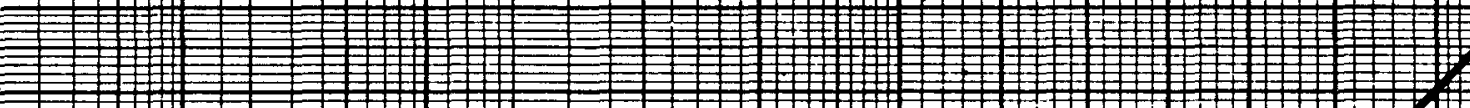

20 \%

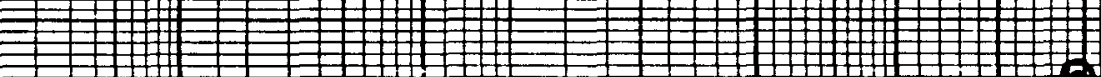

50 2

$0.1_{0.01}$

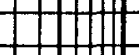

$10+100100$

$\begin{array}{lll}0.050 .1 & 0.2\end{array}$

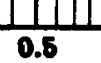

2

Cumulative Per Cent of Activity Less than the Stage Median Value 
$2 x$

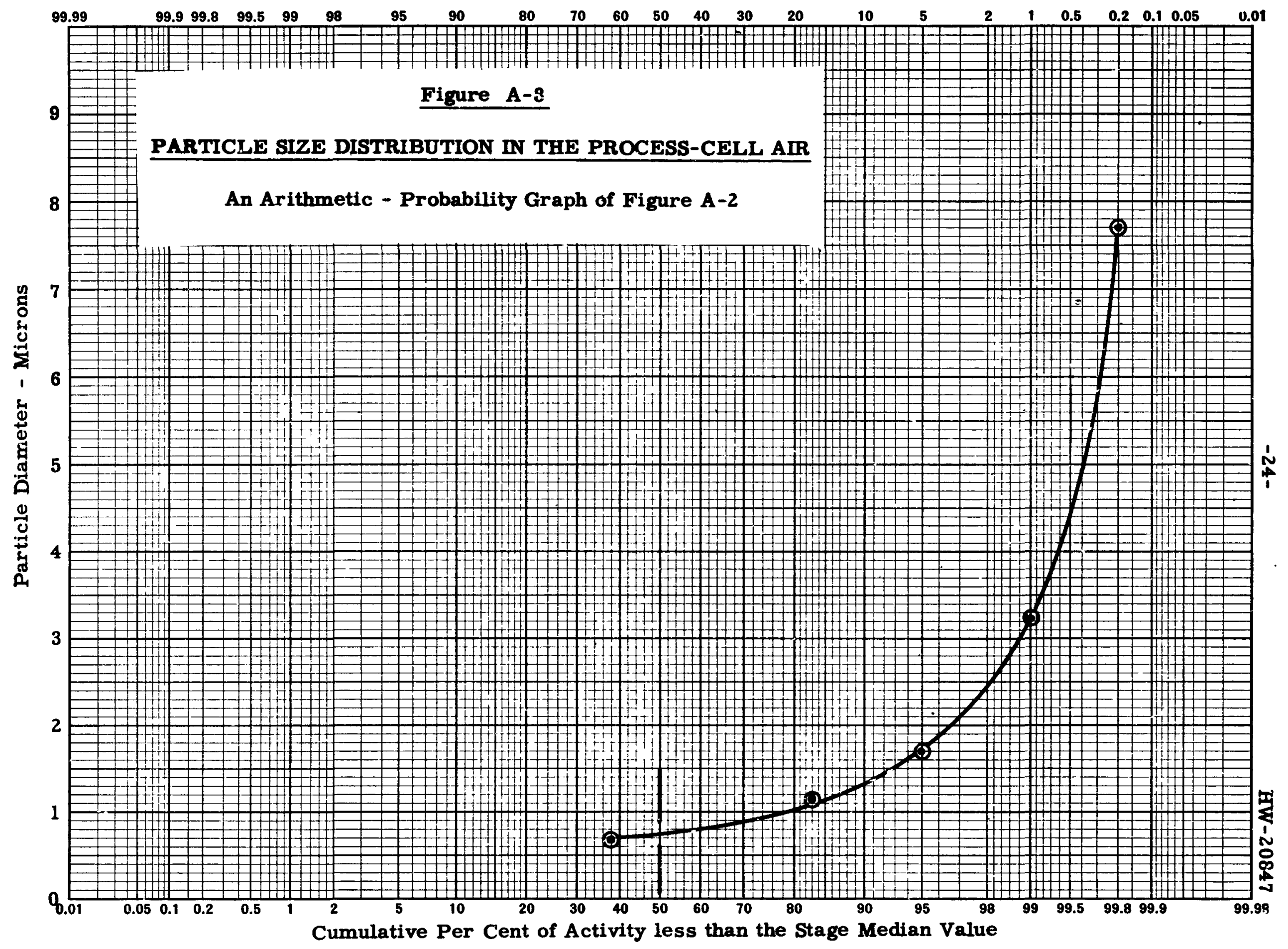




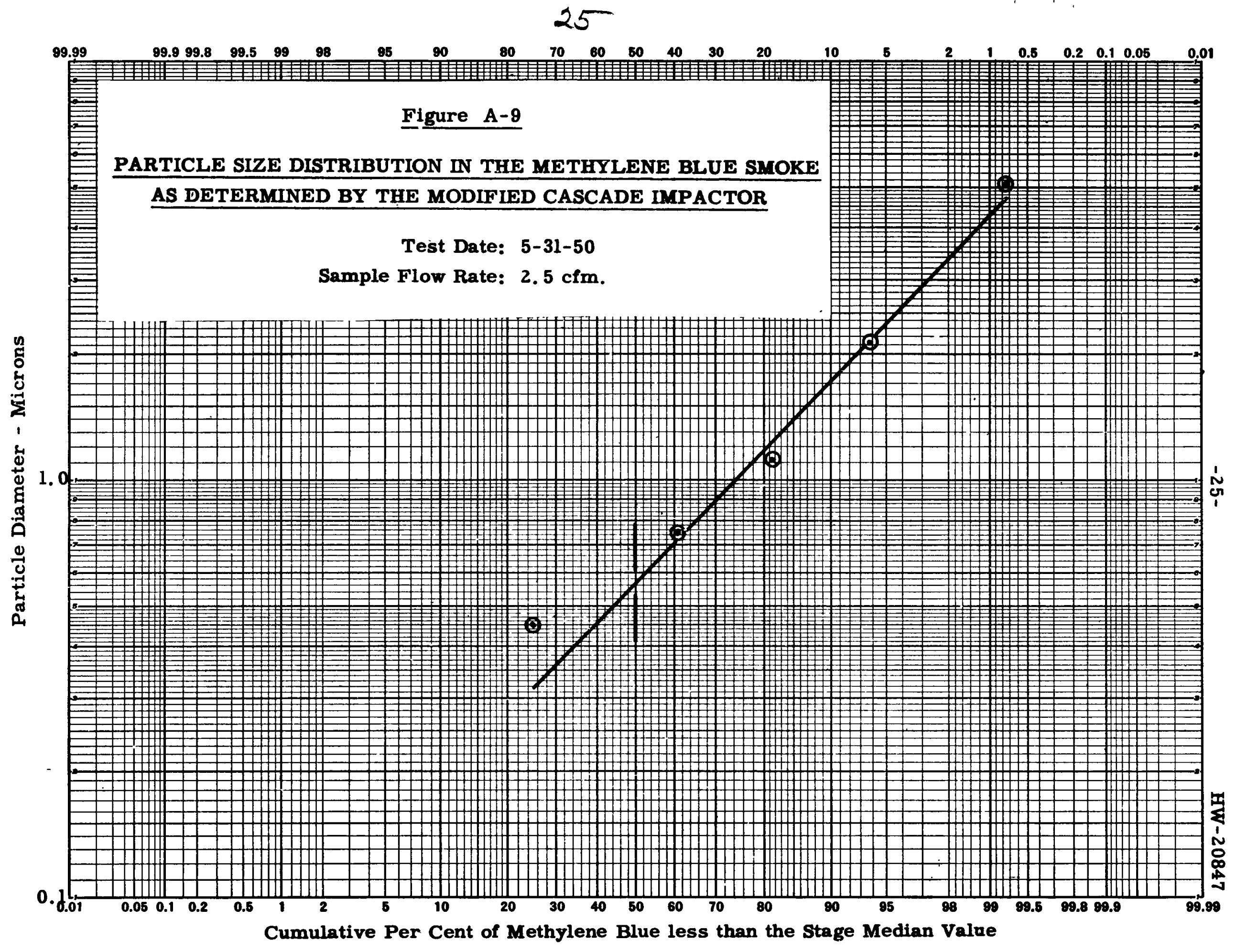


26

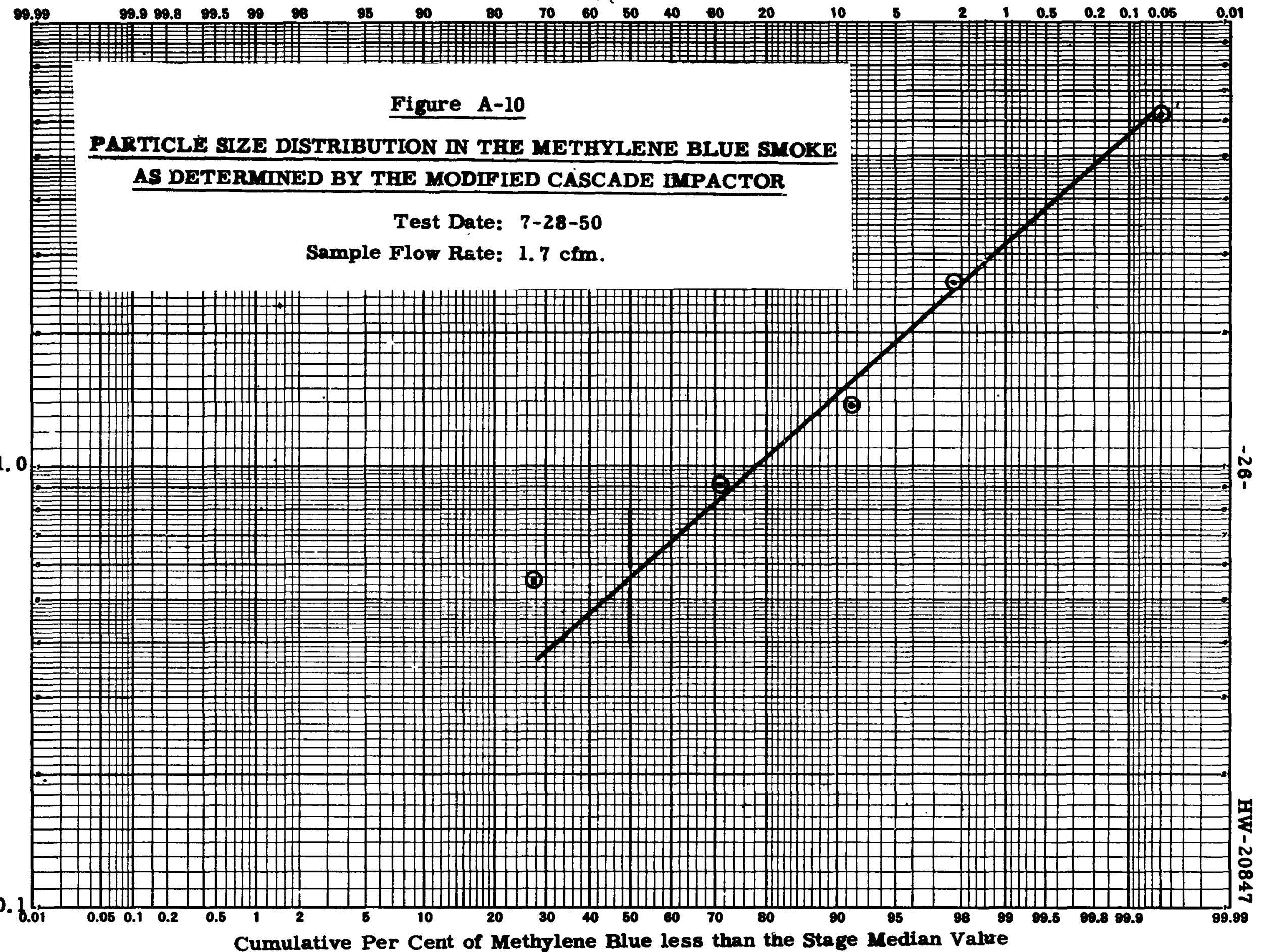

Cumulative Per Cent of Methylene Blue less than the Stage Median Value 
2)

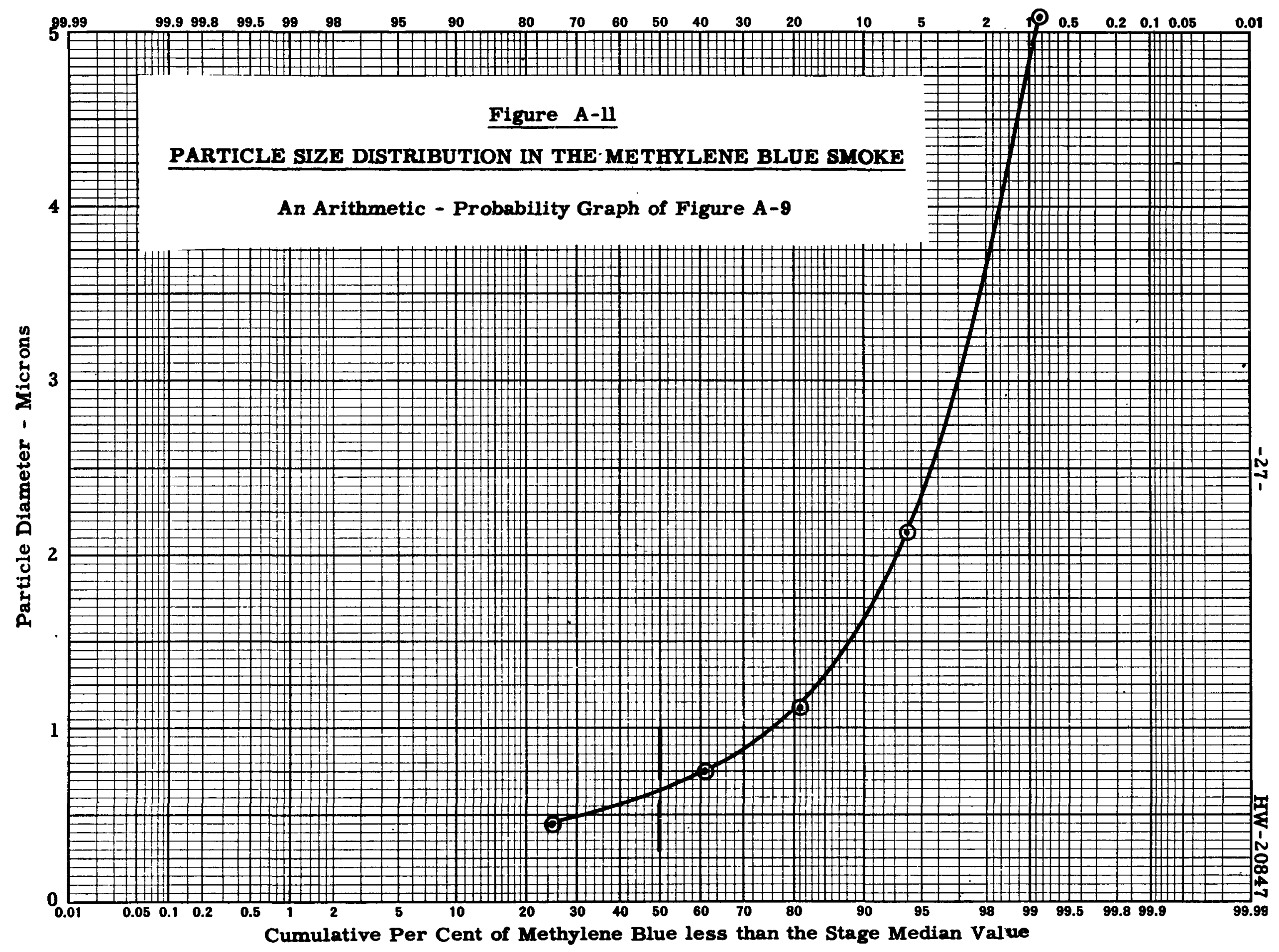


Figure B-1

GRAPH OF PRISSURE DBDP

VS. SUPERFICIAL VELOCITY

No. 55 Fiberglas, $p_{p}=3.0$ pcf.

$X-L=12$ inches

O - L -24 inches

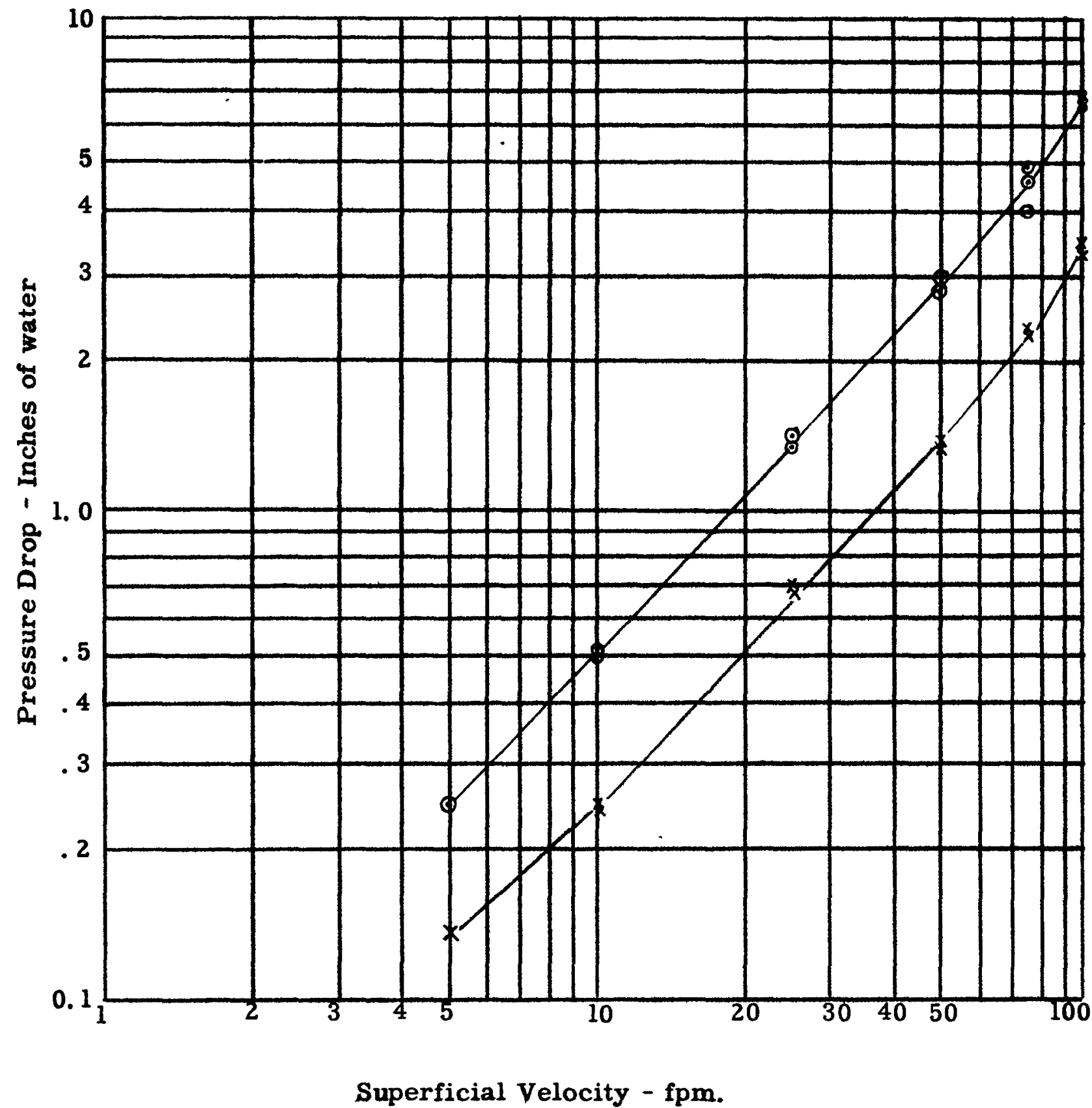


Figure B-2

GRAPH OF PRESSURE DROP

VS. SUPERFICIAL VELOCITY

No. 55 Fiberglas, $P_{P}=6.0 \mathrm{pcf}$ 。

$$
\begin{aligned}
& \square-L=5 \text { inches } \\
& X-L=6 \text { inches } \\
& \Delta-L=9 \text { inches } \\
& \odot-L=48 \text { inches }
\end{aligned}
$$

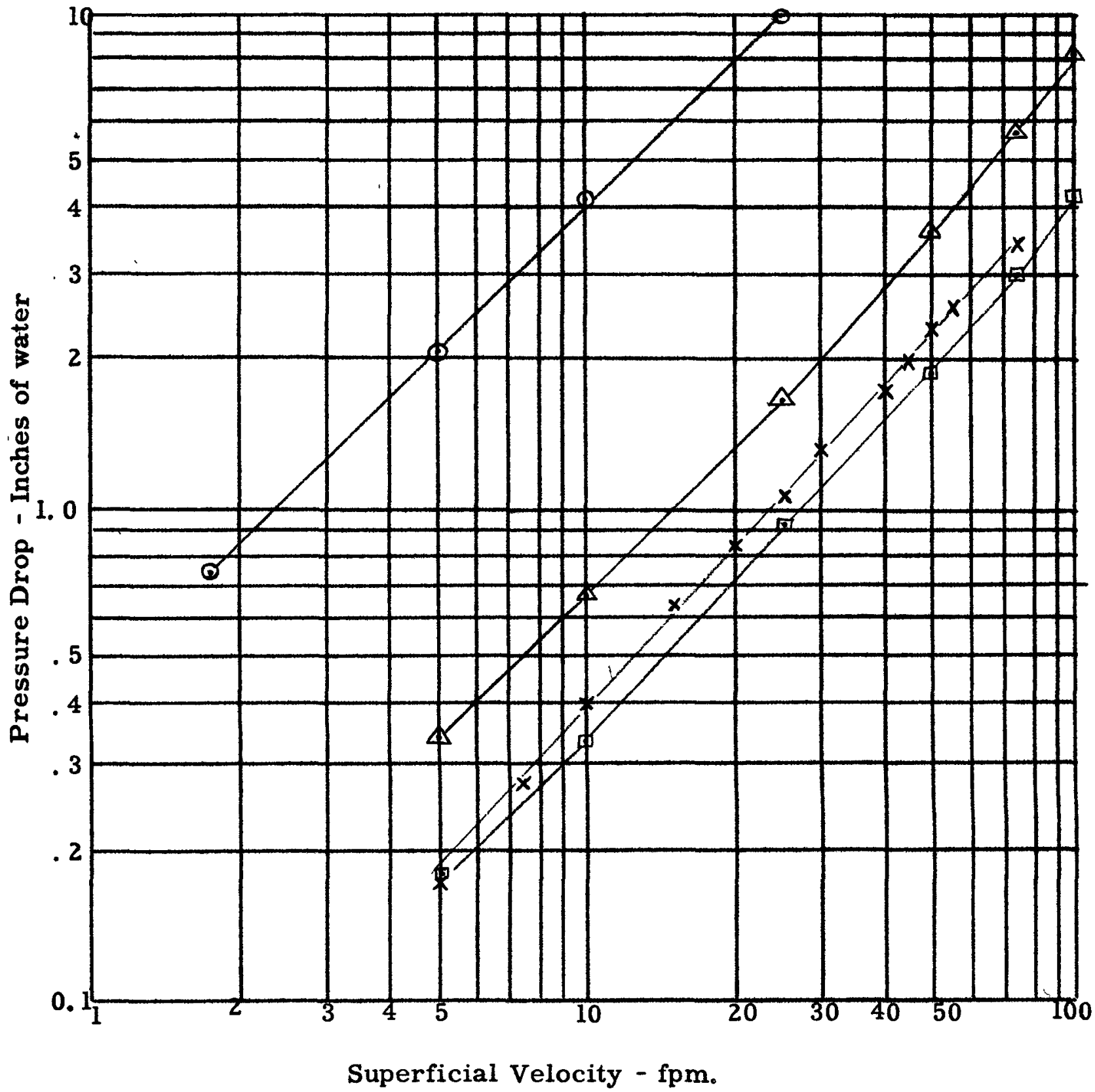


Figure B-3

GRAPH OF PRESSURE DROP

VS. SUPERFICIAL VELOCITY

No. 55 Fiberglas, $\rho_{\rho}=9.0$ pof。

$O-L=2$ inches
$\Delta-L=4$ inches
$\square-L=6$ inches

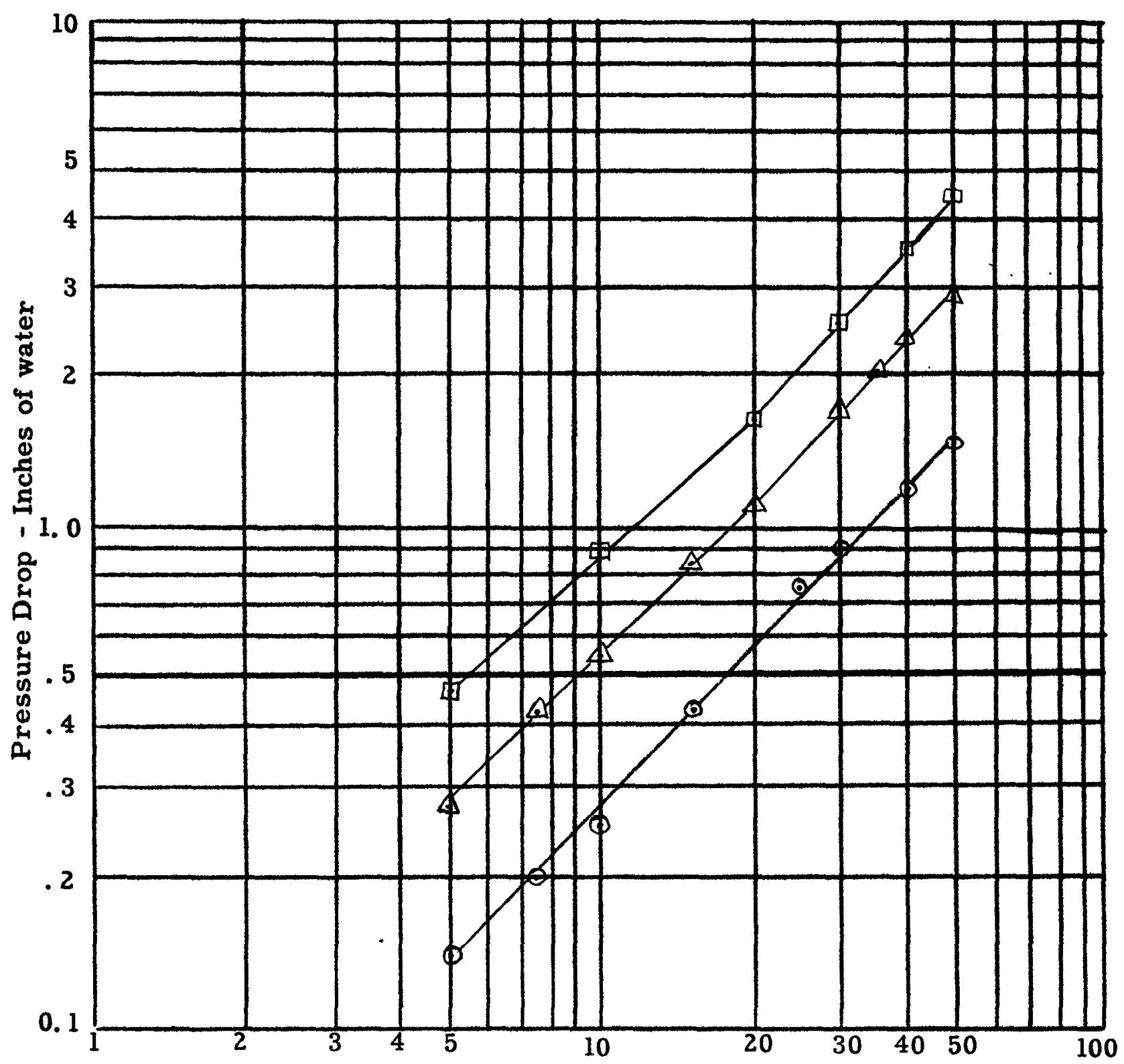

Superficial Velocity - fpm. 
Figure B-4

CROSS-PLOT OF PRESSURE DROP

VS。 BED DEPTH

No. 55 Fiberglas

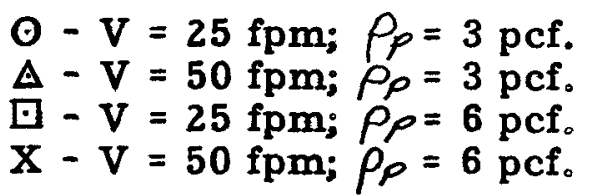

$\otimes-\mathrm{V}=10 \mathrm{fpm} ; \beta=9$ pcf.

$\nabla-V=30 \mathrm{fpm} ; \mathrm{fs}=9$ pcf.

$\Delta-V=50$ fpm; $\rho \rho=9$ pcf。

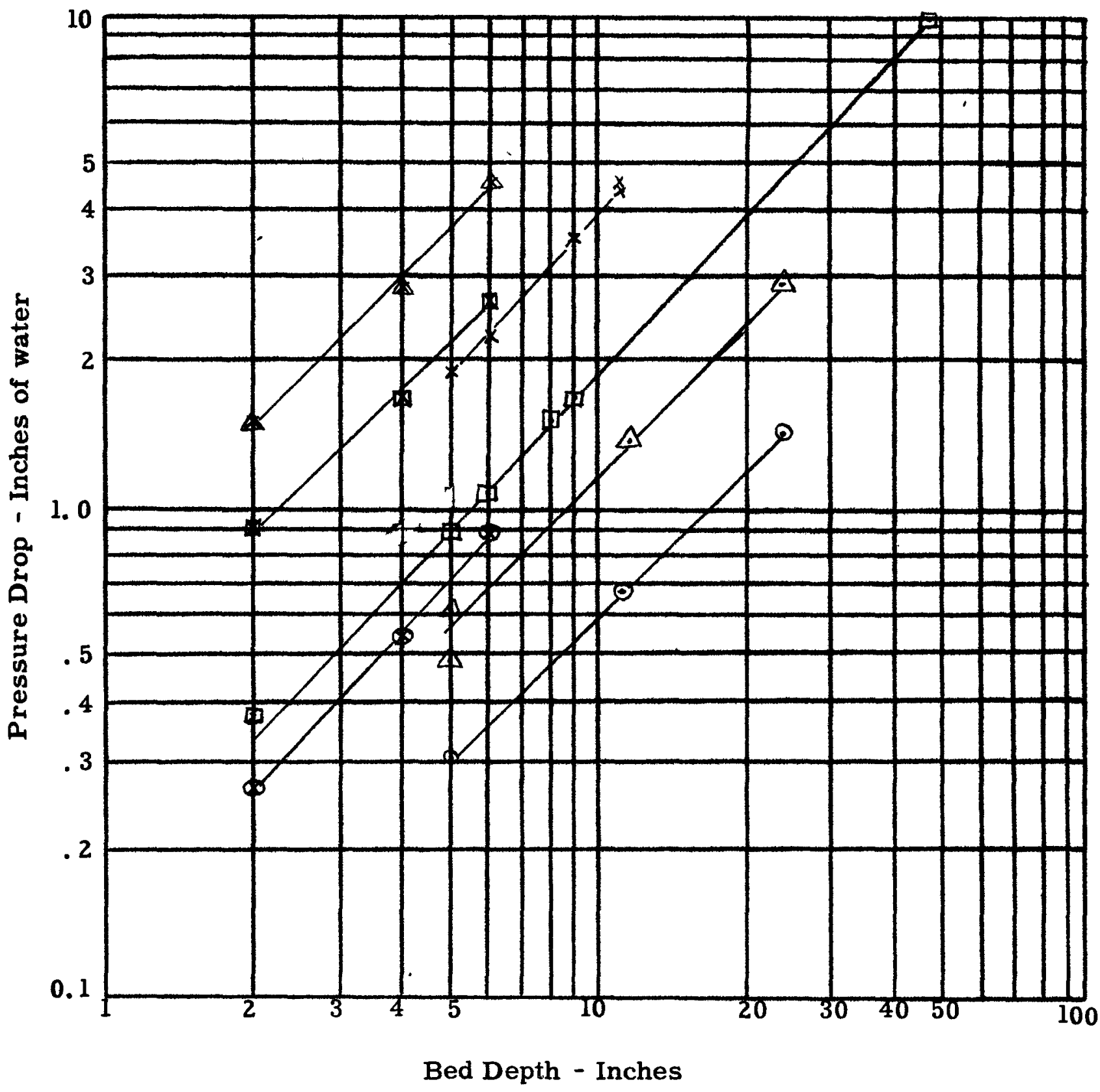


Figure B-5

CROSS-PLOT OF PRESSURE DROP

VS.PACKING DENSITY

No。 55 Fiberglas

$\odot-V=25 \mathrm{fpm}_{2} \mathrm{~L}=6$ inches

$\Delta-\mathrm{V}=50 \mathrm{fpm}_{\diamond} \mathrm{L}=5$ inches

$X-V=50 \mathrm{fpm}, L=10$ inches

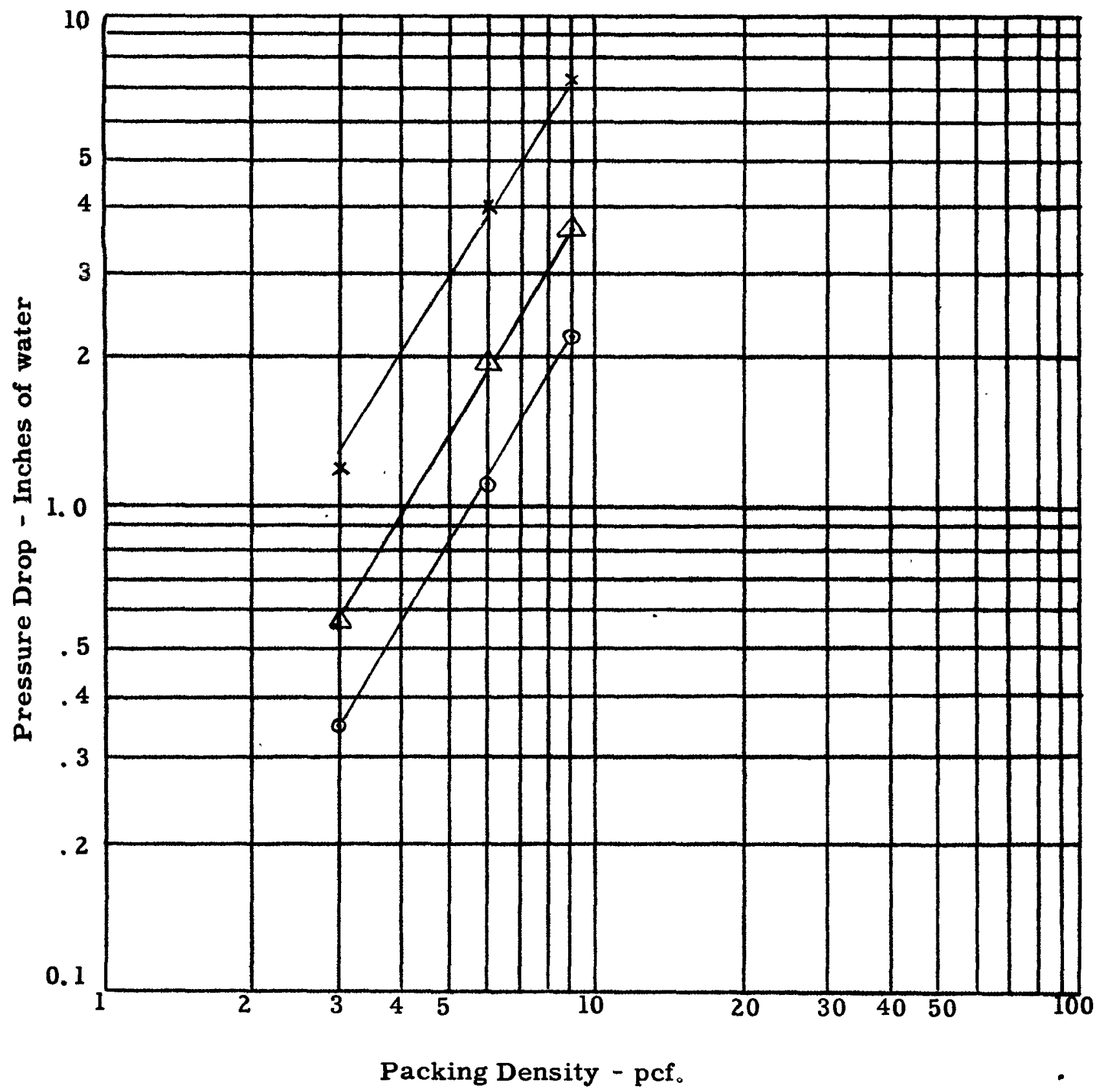


Figure B-6

GRAPH OF PRESSURE DROP

VS. SUPERFICIAL VELOCITY

No. 55 P Fiberglas

$\odot-L=6$ inches; $P_{P}=2.0$ pcf.

$X-L=6$ inches; $p_{p}=3.0$ pcf。

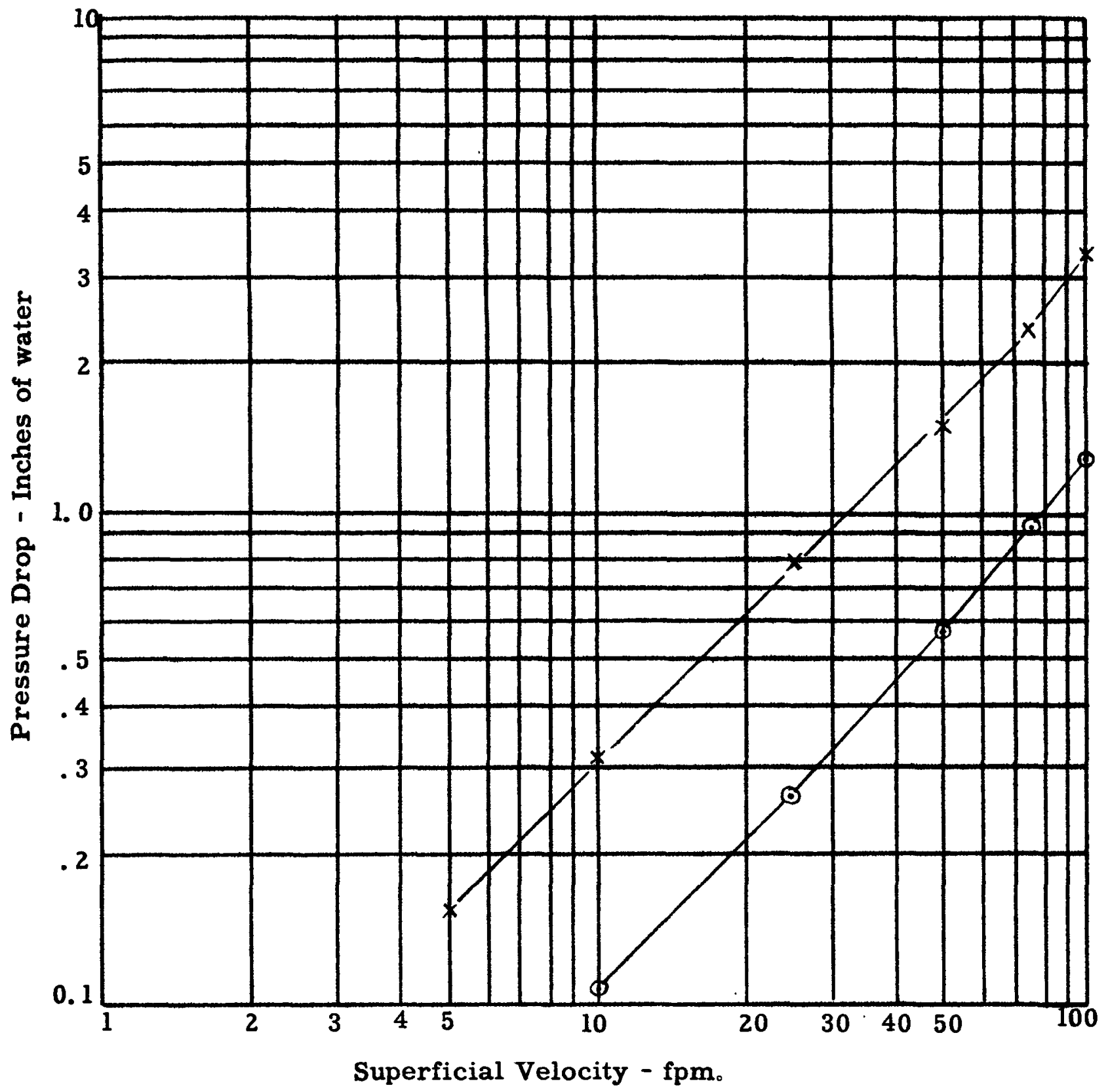


Figure B-7

GRAPH OF PRESSURE DROP

VS. SUPERFICIAL VELOCITY

$$
\begin{aligned}
& \text { AA Fiberglas } \rho \rho=0.6 \text { pcf. } \\
& O-L=1.5 \text { inches } \\
& X-L=1.0 \text { inch } \\
& \Delta-L=0.5 \text { inch }
\end{aligned}
$$

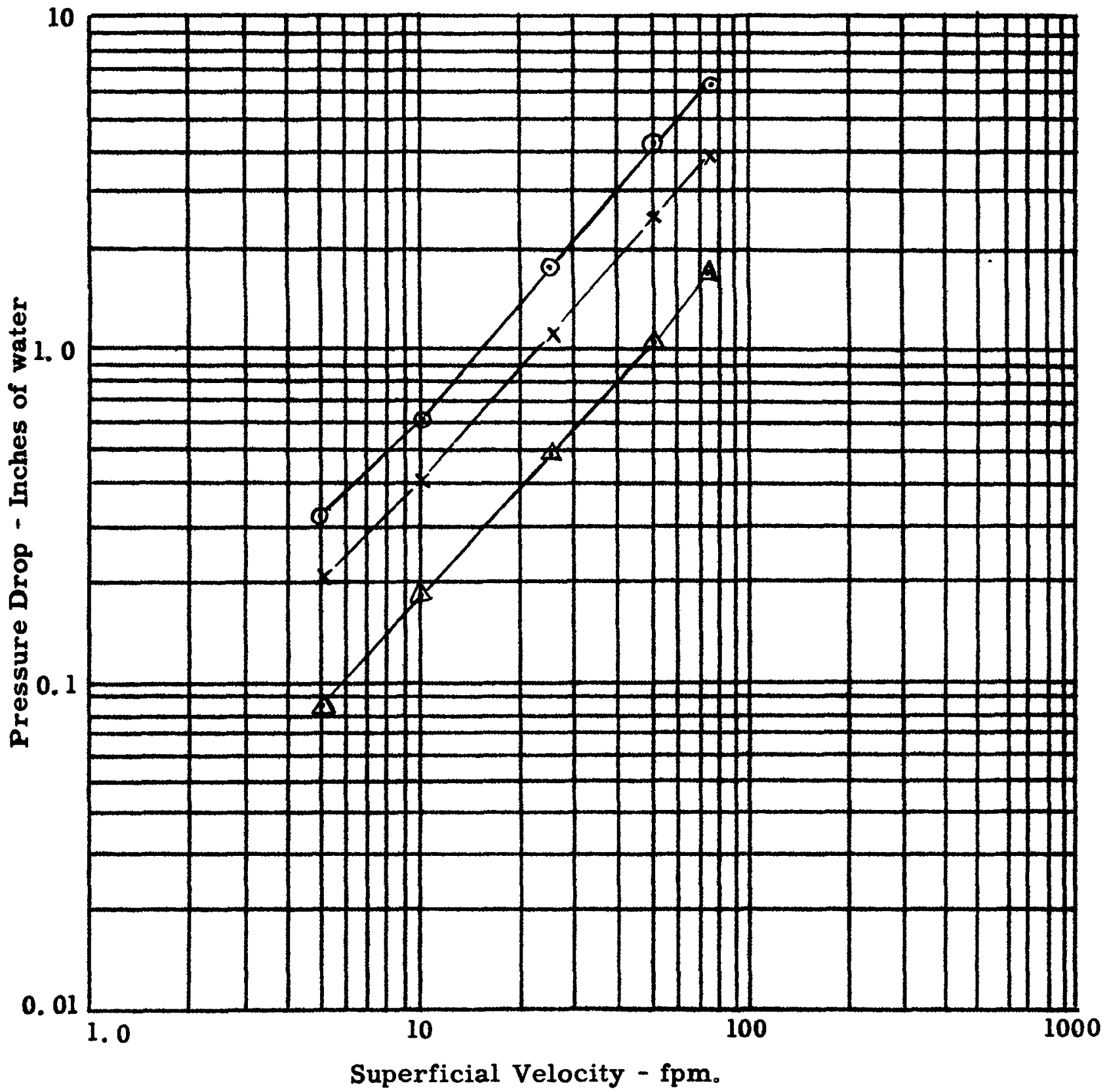


Figure B-8

GRAPH OF PRESSURE DROP

VS. SUPERFICIAL VELOCITY

AA Fiberglas, $\rho_{p}=1.2$ pcf.

O $-\mathrm{L}=1.00 \mathrm{inch}$

$X-L=0.75$ inch

$\boldsymbol{a}-\mathrm{L}=0,50$ inch

$\Delta-\mathrm{L}=025 \mathrm{inch}$

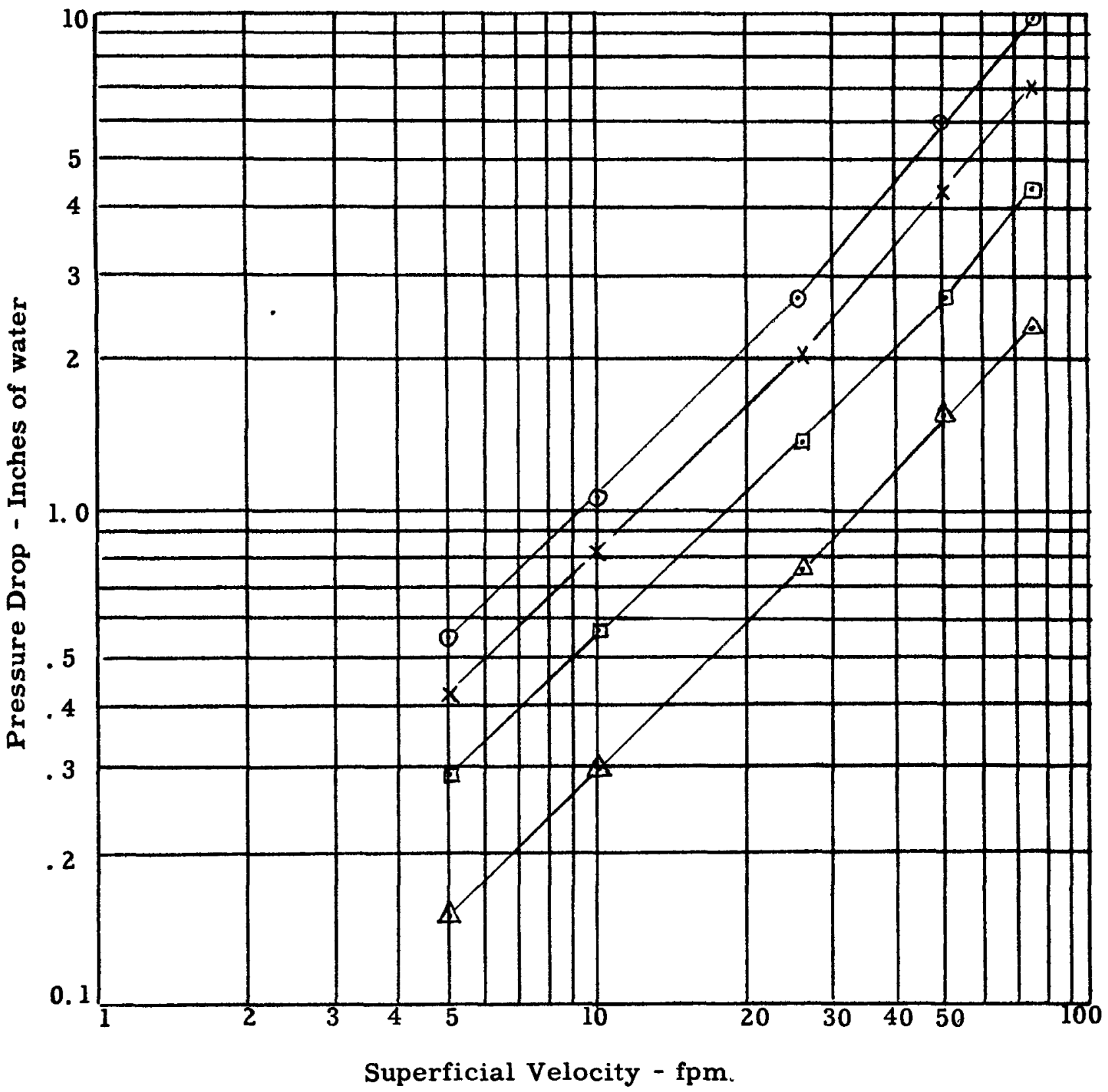


Figure B-9

GRAPH OF PRESSURE DROP

VS. SUPERFICIAL VELOCITY

AA Fiberglas, $P_{\rho}=2.4$ pcf.

$O-L=1.00$ inch

$X-L=0.75$ inch

$\square-\mathrm{L}=0.50$ inch

$\Delta-\mathrm{L}=0.25 \mathrm{inch}$

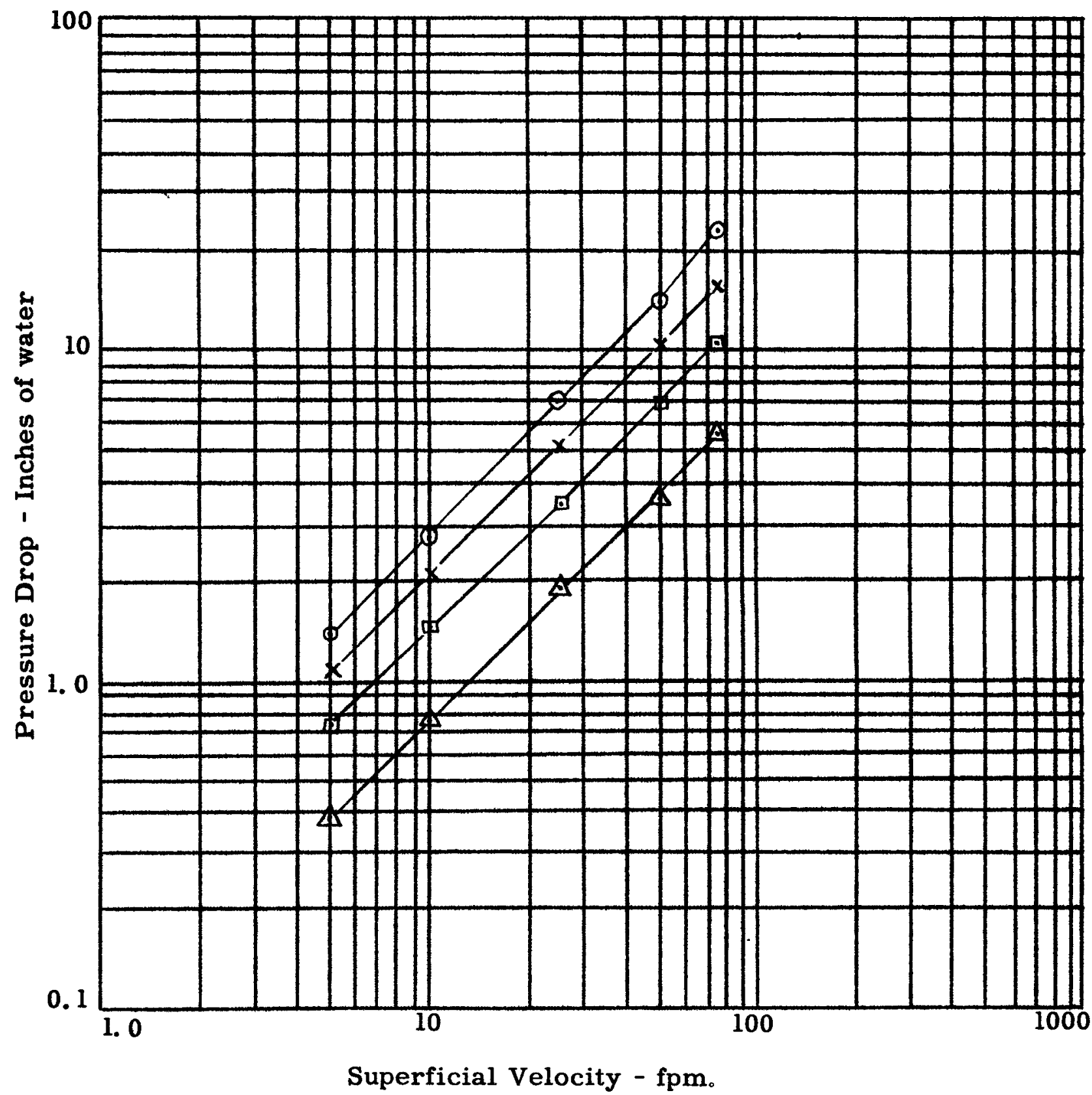


Figure B-10

CROSS-PLOT OF PRESSURE DROP

VS。 BED DEPTH

AA Fiberglas

$\odot-\mathrm{V}=50 \mathrm{fpm} ; \varphi_{p}=1.2 \mathrm{pcf}$.

$\Delta-V=10 \mathrm{fpm} ; \quad e_{P}=1.2 \mathrm{pcf}$

$\square-V=10 \mathrm{fpm} ; \rho_{p}=0.6$ pcf.

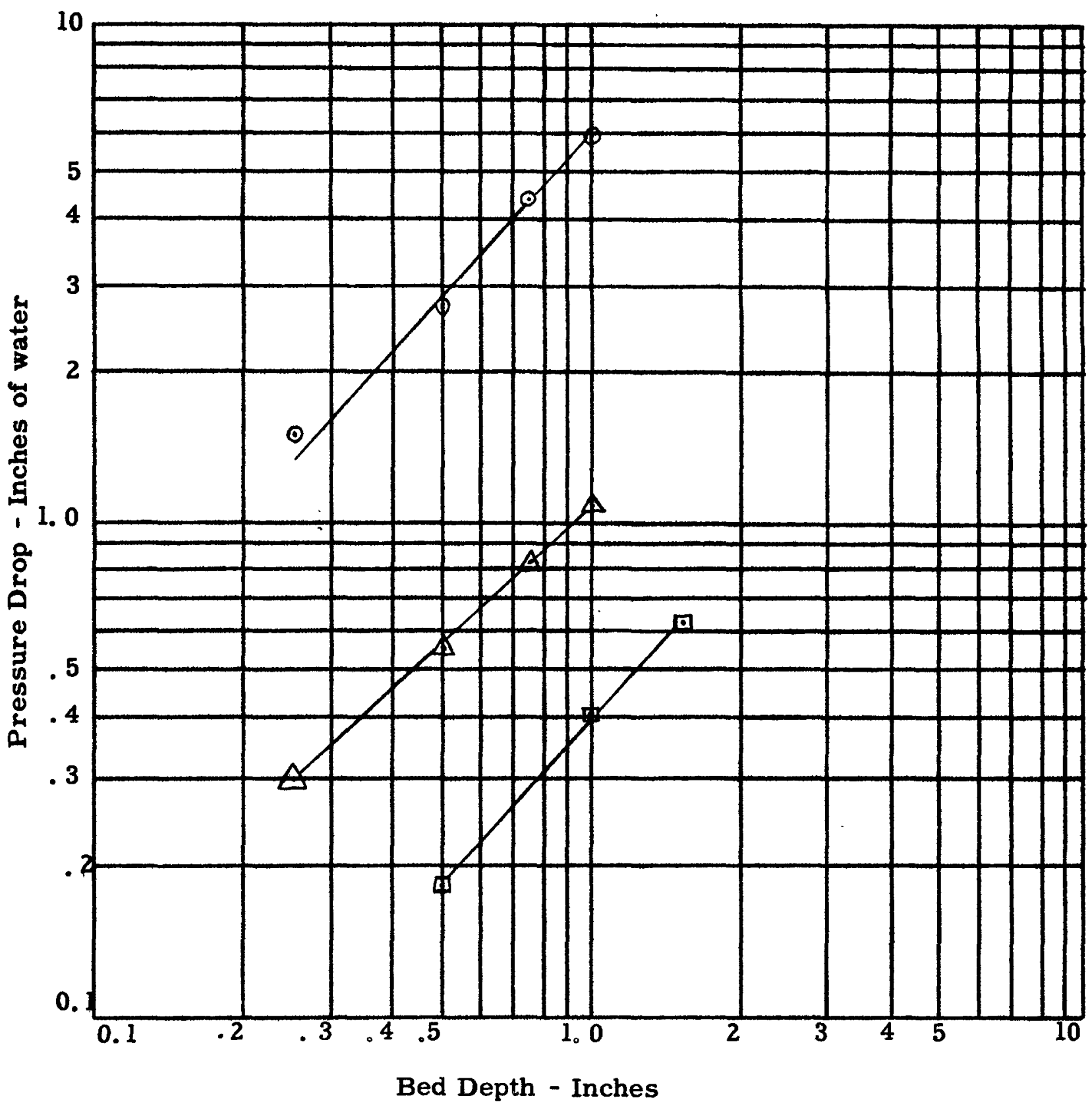


Figure B-11

CROSS-PLOT OF PRESSURE DROP

VS. PACKING DENSITY

AA Fiberglas

$O-L=0.25$ inch; $V-5$ fpm.
$\Delta-L=0.25$ inch; $V-10$ fpm.
$X-L=0.50$ inch; $V-10$ fpm.
$\square-L=0.50$ inch; $V-20$ fpm.

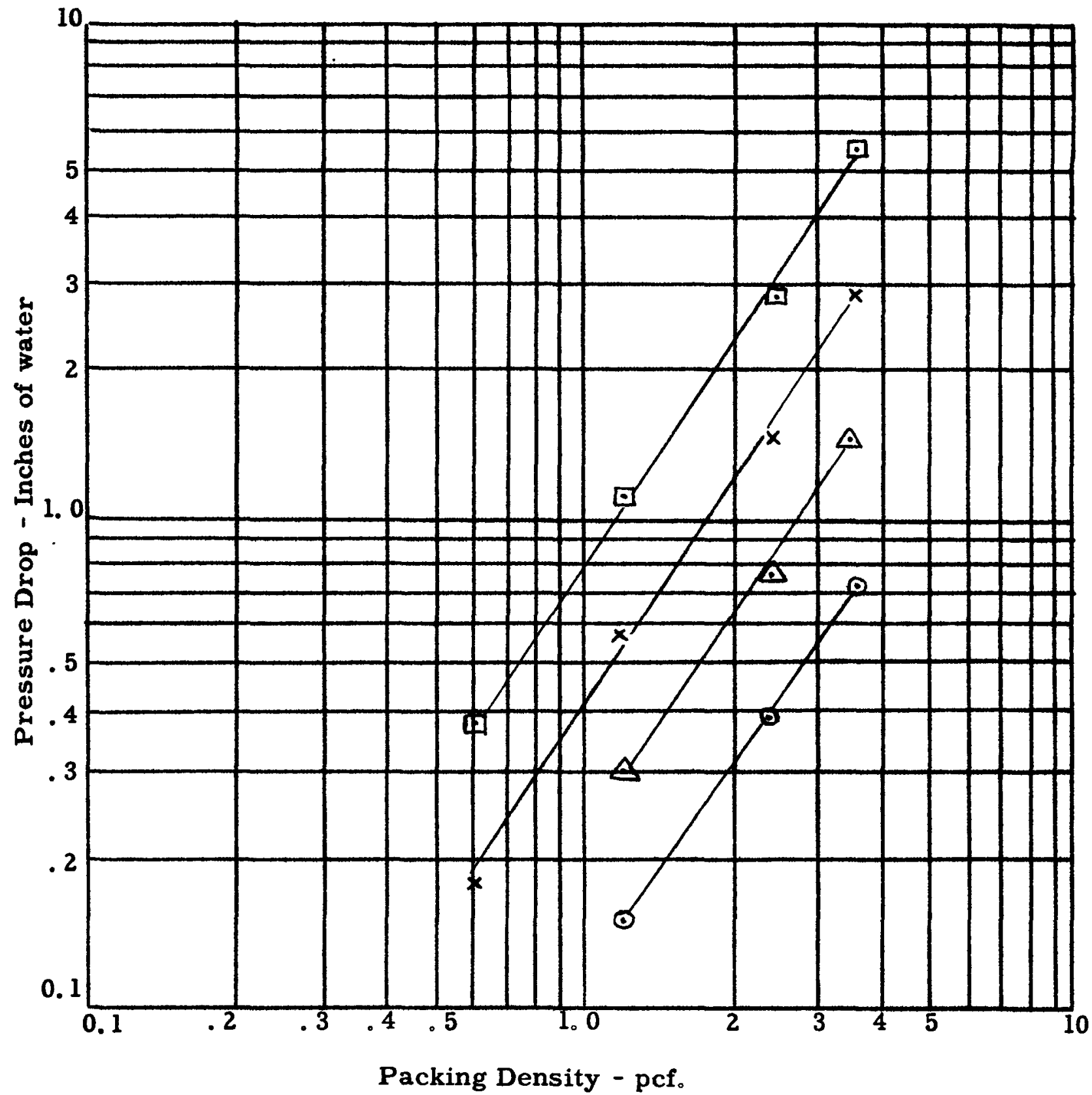


Figure B-12

GRAPH OF PRESSURE DROP

VS。SUPERFICIAL VELOCITY

B Fiberglas

$O-\mathrm{L}=1.0$ inch, $\rho_{\rho}=1.4 \mathrm{pcf}_{\text {。 }}$

$\Delta-L=1.0$ inch $P_{P}=0.7$ pcf。

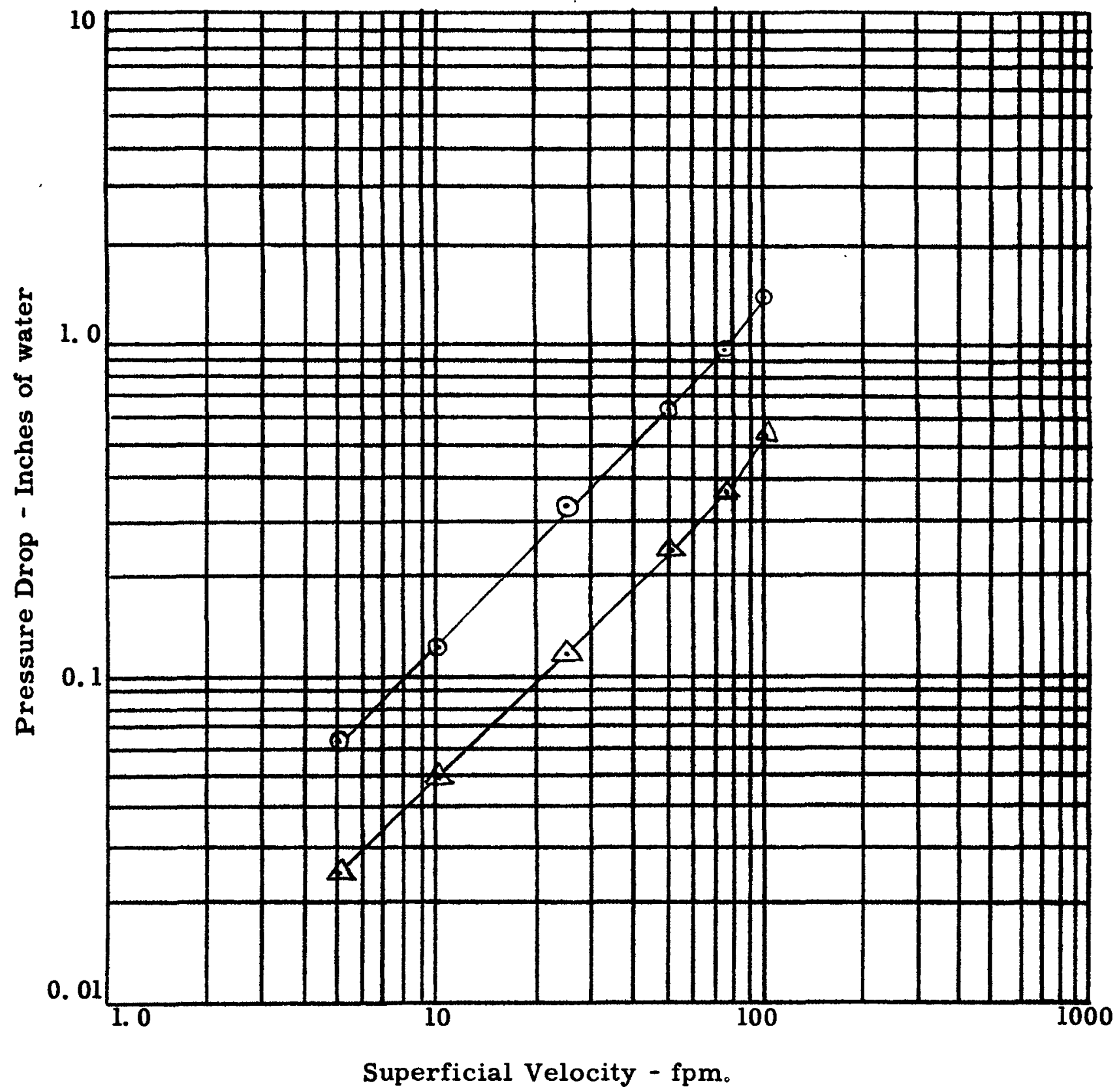


Figure B-13

GRAPH OF PRESSURE DROP

VS. SUPERFICIAL VELOCITY

$B$ ( $L$ and $R$ ) Fiberglas

$$
\begin{aligned}
& e_{P}=3.5 \text { pcf. } \\
& L=1.0 \text { inch }
\end{aligned}
$$

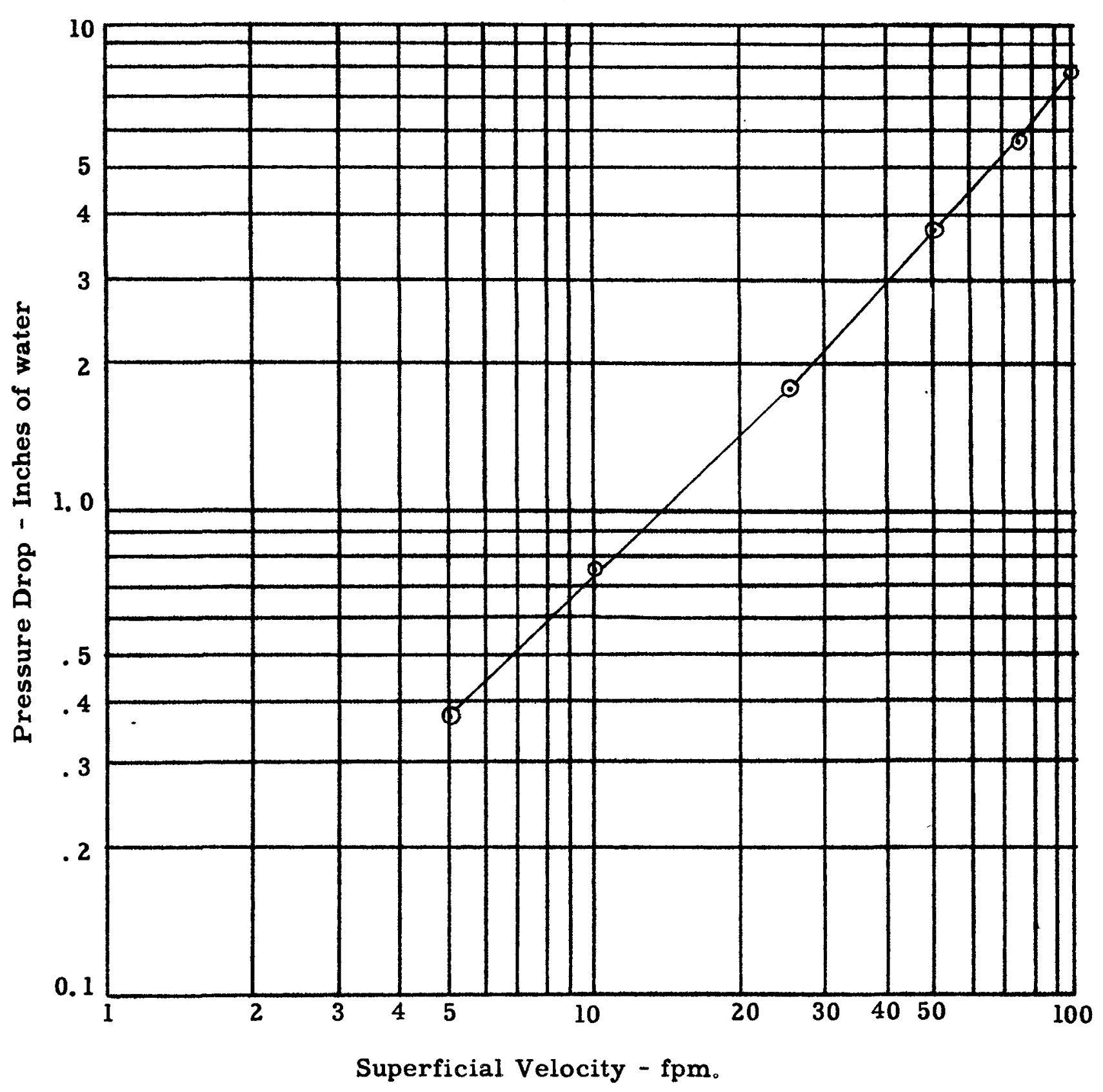


Figure B-14

GRAPH OF PRESSURE DROP

VS. SUPERFICIAL VELOCITY

No. 450 Fiberglas

$e_{P}=5.7$ pcf

$L=8$ inches

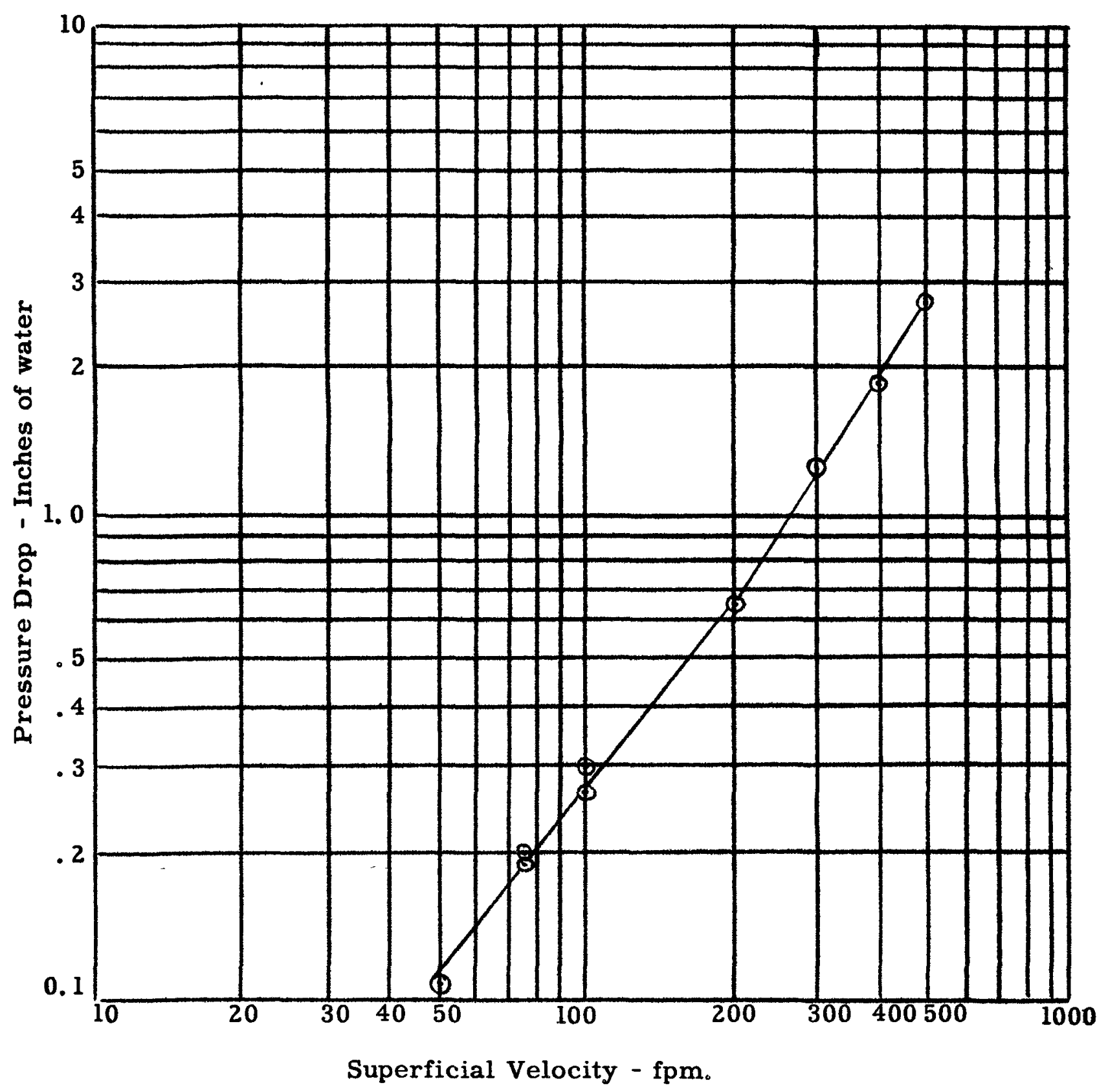


Figure B-15

GRAPH OF PRESSURE DROP

VS. SUPERFICIAL VELOCITY

No. 600 Fiberglas

$e_{P}=5$. 1 pcf。

$\mathrm{L}=7$ inches

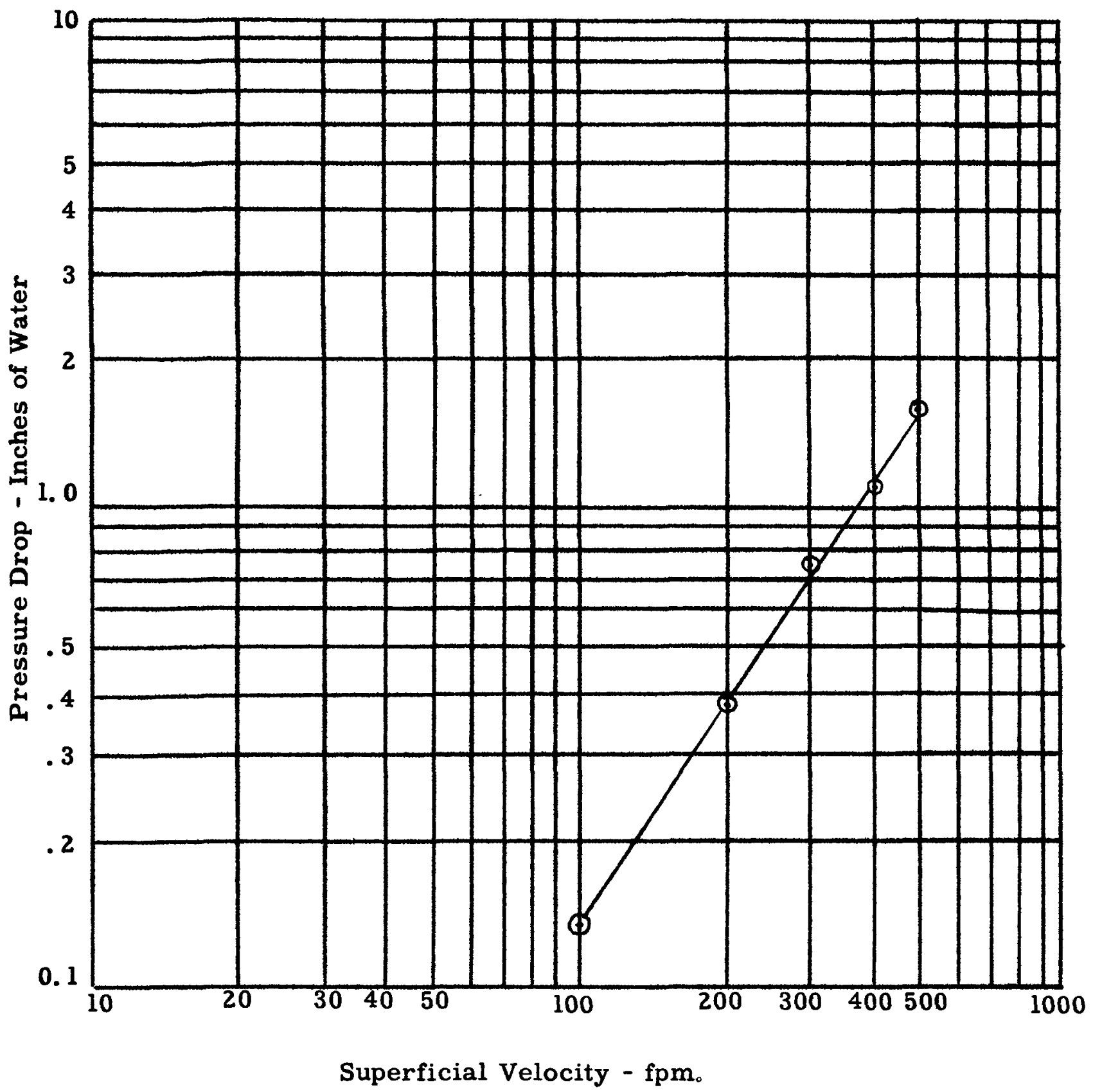


Figure B-16

GRAPH OF PRESSURE DROP

VS。SUPERFICIAL VELOCITY

No. 800 Fiberglas

$\varphi_{\rho}=6.6 \mathrm{pcf}_{\text {。 }}$

$L=8$ inches

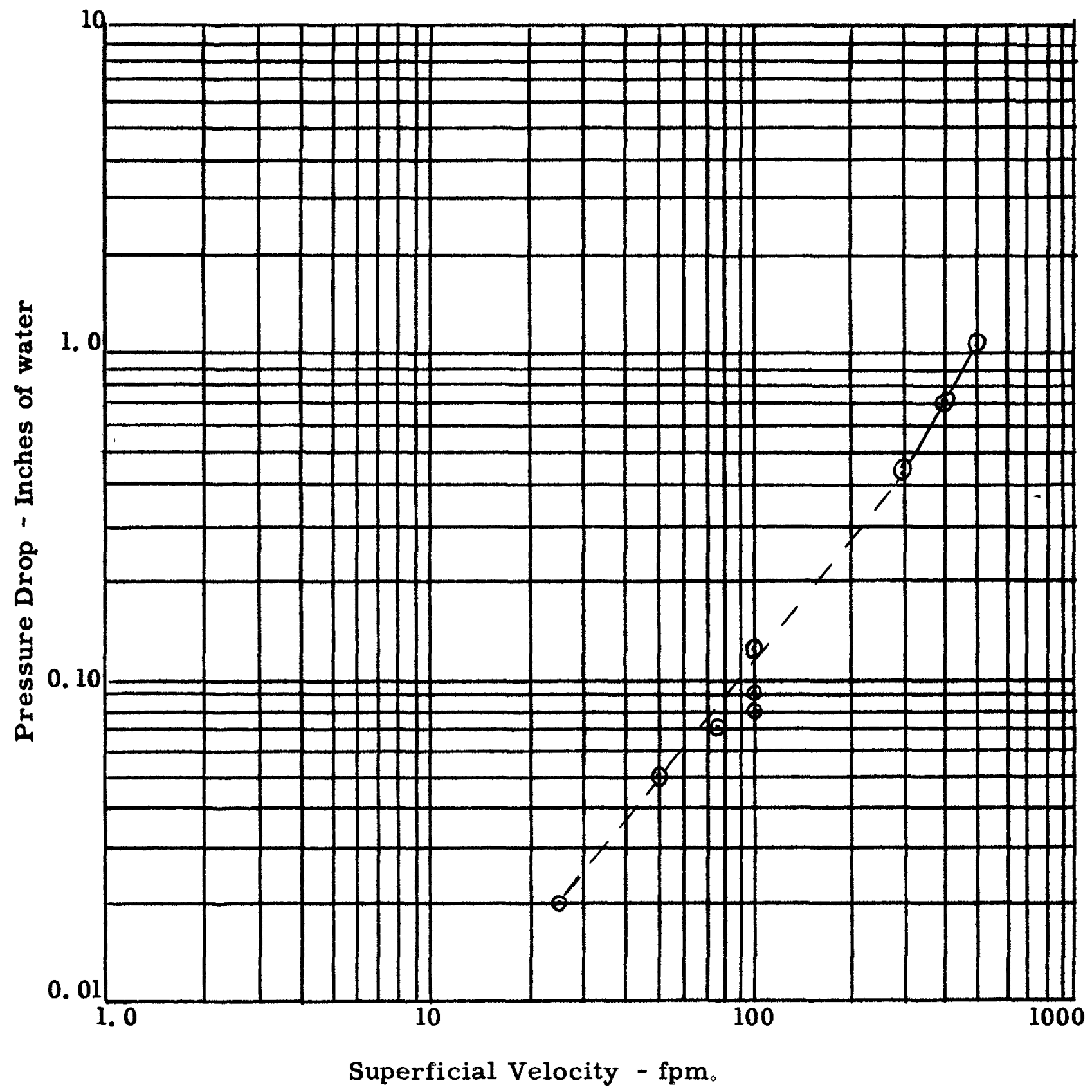


Figure B-17

GRAPH OF PRESSURE DROP

VS. SUPERFICIAL VELOCITY

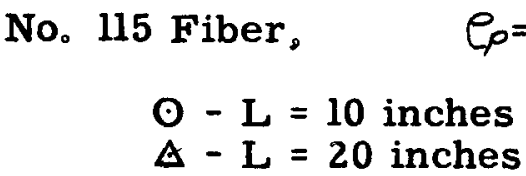

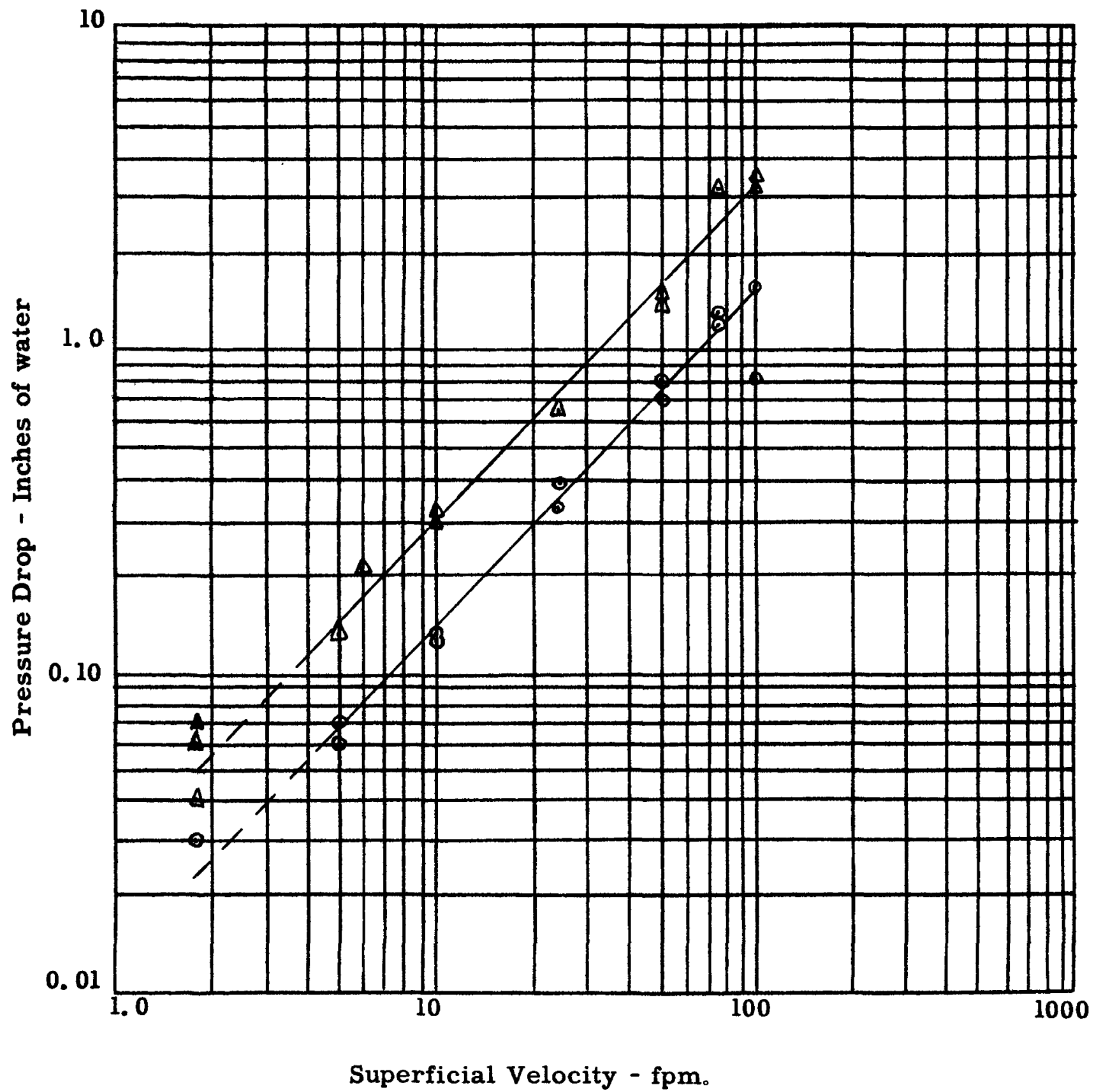


Figure B-18

GRAPH OF PRESSURE DROP

VS. SUPERFICIAL VELOCITY

No. $115 \mathrm{~K}$ Fiberglas

曰 $L=6$ inches, $\rho_{\rho}=6.0$ pcf

$\odot-L=12$ inches, $\rho_{\rho}=6.0$ pcf。

$\odot-L=6$ inches,

$\Delta-L=24$ inches, $\rho_{\rho}=6.0$ pcf。

$X-L=12$ inches, $\quad \rho_{\rho}=1.5$ pcf.

$\nabla-L=18$ inches, $e_{p}=1.5$ pcf.

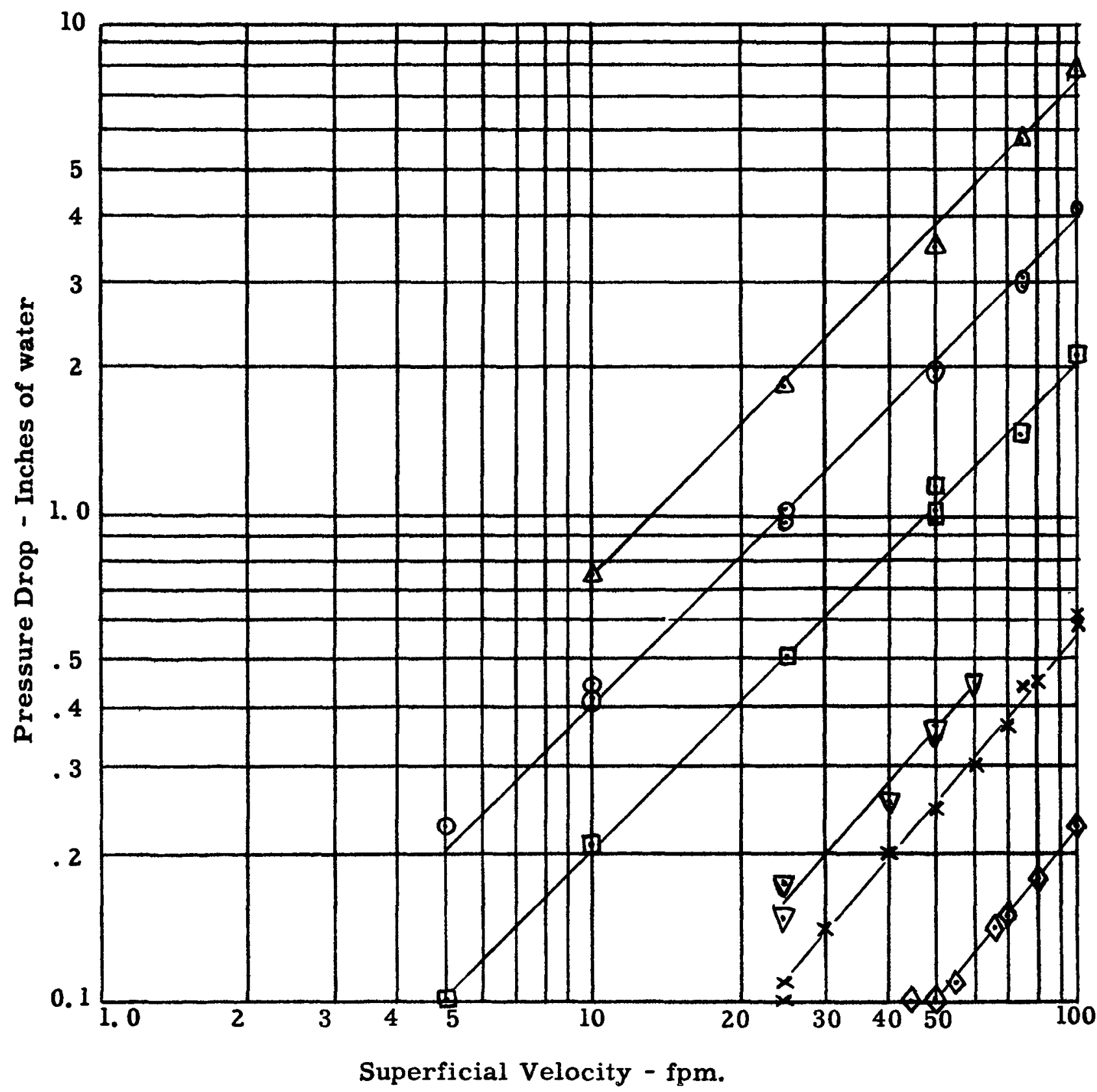


Figure B-19

GRAPH OF PRESSURE DROP VS。SUPERFICIAL VELOCITY

NO. 115K FIBERGLAS
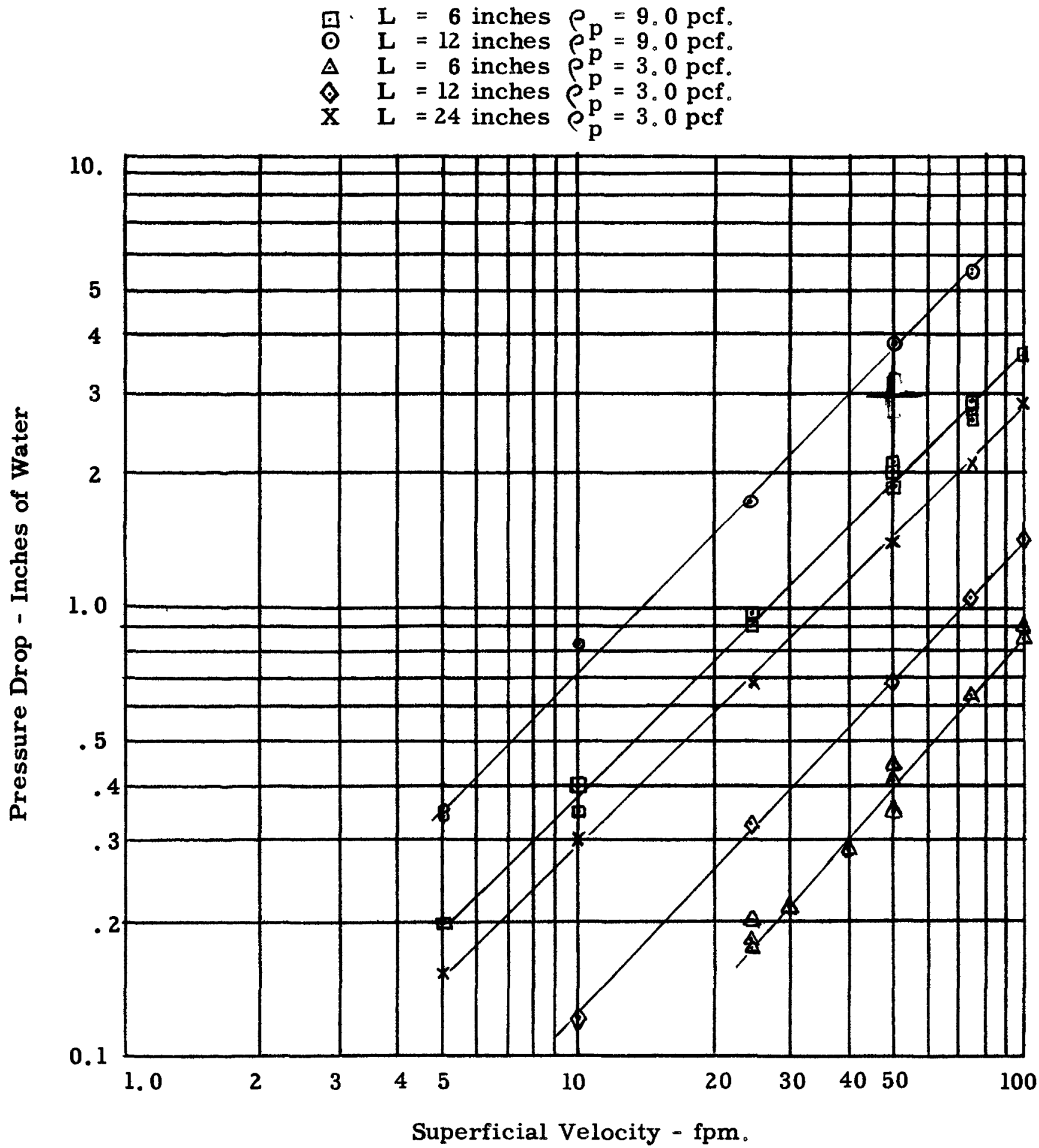
Figure B-20

GRAPH OF PRESSURE DROP VS. BED DEPTH

NO. 115K FIBERGLAS

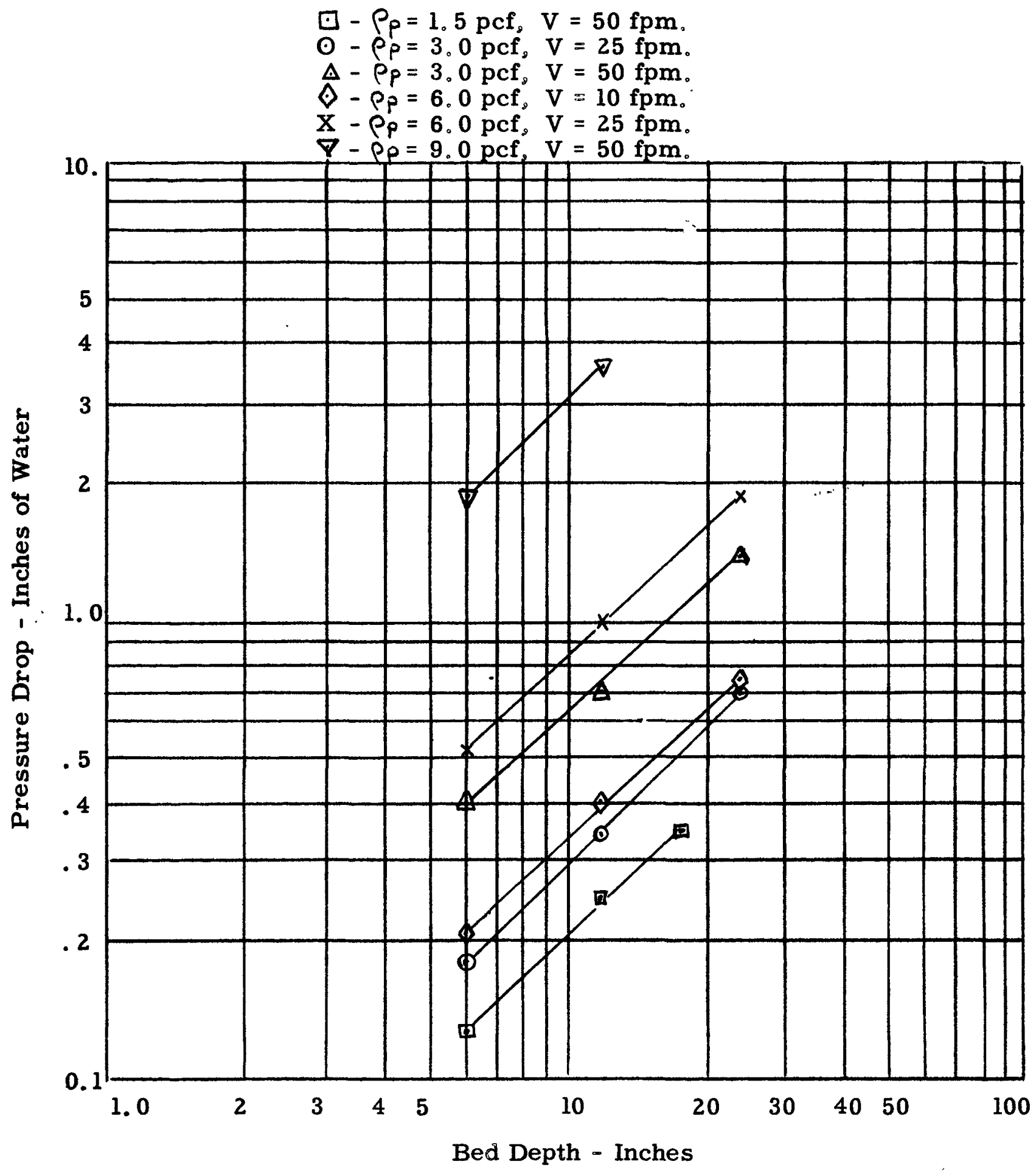


Figure B-21

GRAPH OF PRESSURE DROP VS。 PACKING DENSITY NO. 115K FIBERGLAS

$\square \mathrm{L}=6$ inches, $\mathrm{V}=25 \mathrm{fpm}$.

$\odot \mathrm{L}=12$ inches, $\mathrm{V}=10 \mathrm{fpm}$.

$\Delta \mathrm{L}=12$ inches, $V=25 \mathrm{fpm}$.

$\diamond \mathrm{L}=12$ inches, $\mathrm{V}=50 \mathrm{fpm}$.

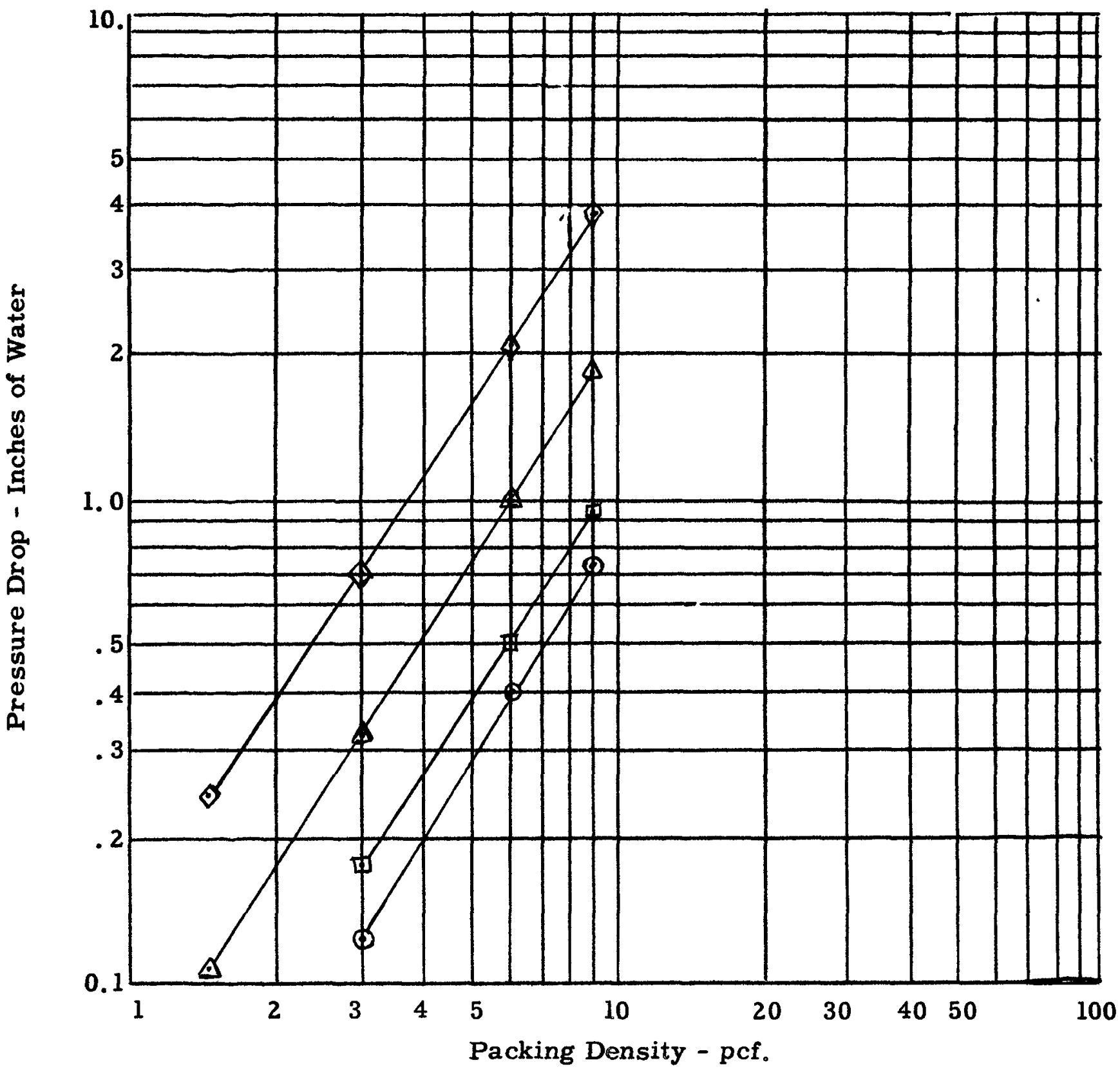


so

vS. DECONTAMINATION FACTOR

\section{Figure C-1}

RAPH OF COLLECTION EFFICIENCY

40

3.0

10.0

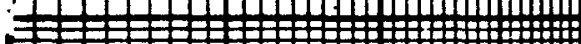

䏠

弹

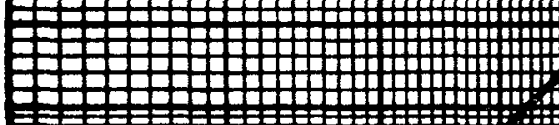

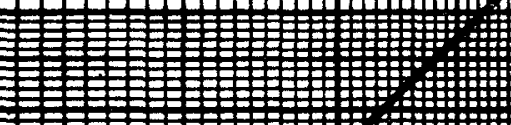

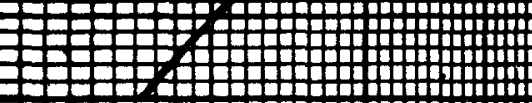

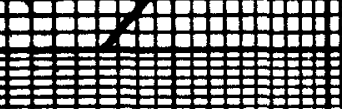
行

2.0 (I)

浑 沏

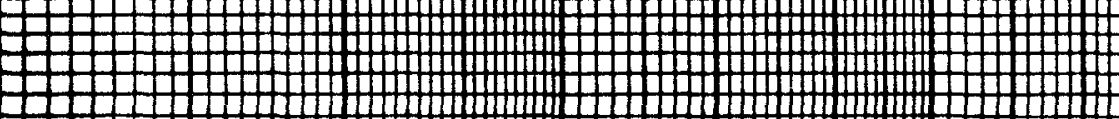
拈 1000.01 49

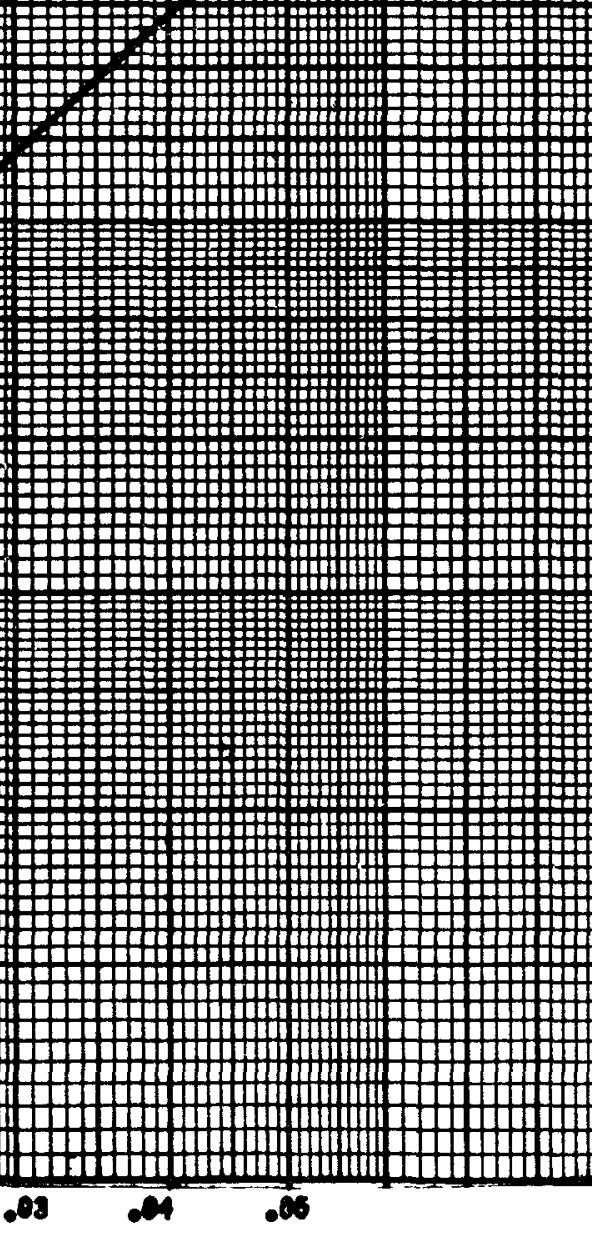

0.10

\section{1}

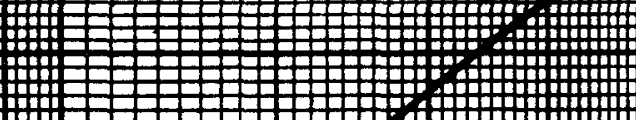

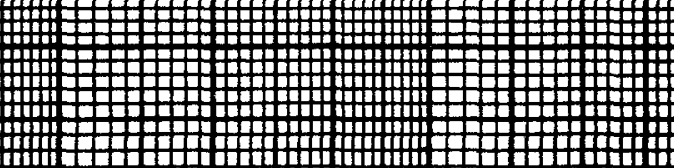
m (m) † 约

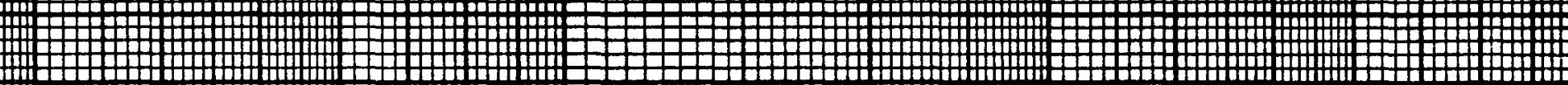
m m 
Figure C-2

GRAPH OF DECONTAMINATION FACTOR

VS. SUPERFICIAL VELOCITY

No. 55 Fiberglas, $\rho_{p}=3.0$ pcf.

$\odot-L=12$ inches

$X-L=24$ inches

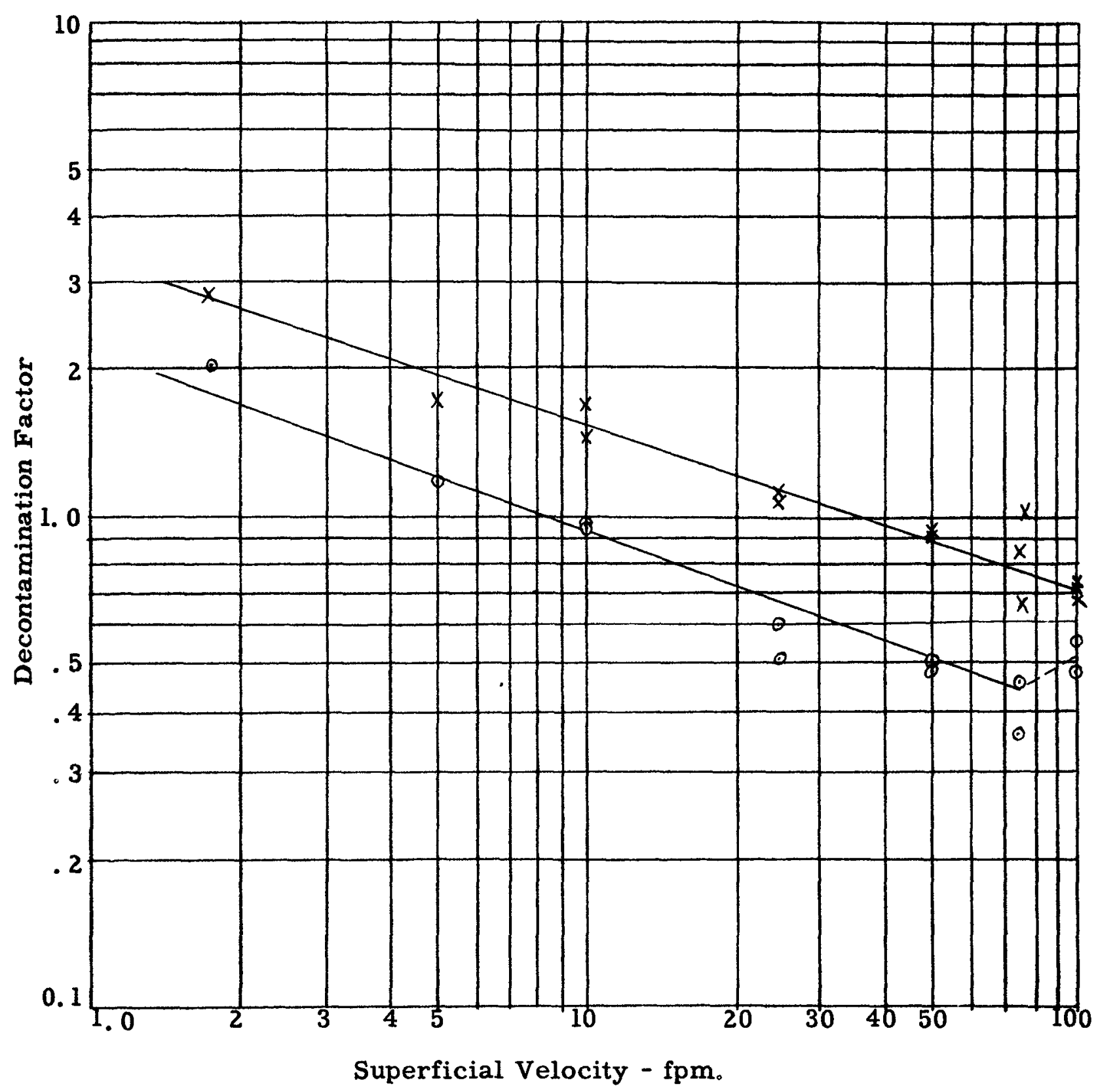




$$
-51-\quad 52
$$

Figure C-3

GRAPH OF DECONTAMINATION FACTOR

VS. SUPERFICIAL VELOCITY

No. 55 Fiberglas, $\rho_{\mathrm{p}}=4.5$ pcf.

$\mathrm{L}=6$ inches

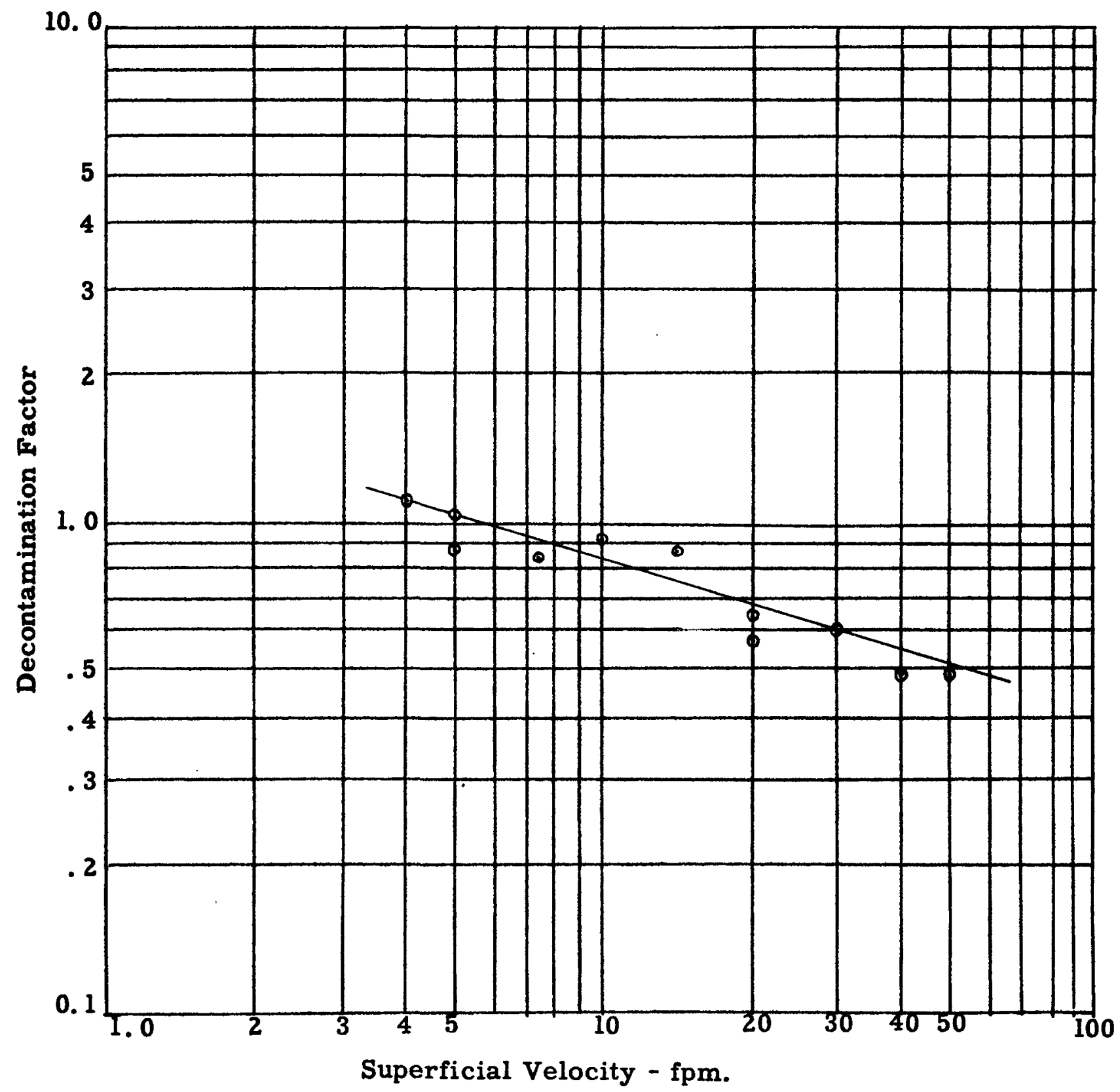


$-52-\quad 53$

Figure C-4

GRAPH OF DECONTAMINATION

FACTOR VS. SUPERFICIAL VELOCITY

No. 55 Fiberglas, $\rho_{p}=6.0$ pcf.

$$
\begin{aligned}
& O-L=5 \text { inches } \\
& X-L=9 \text { inches }
\end{aligned}
$$

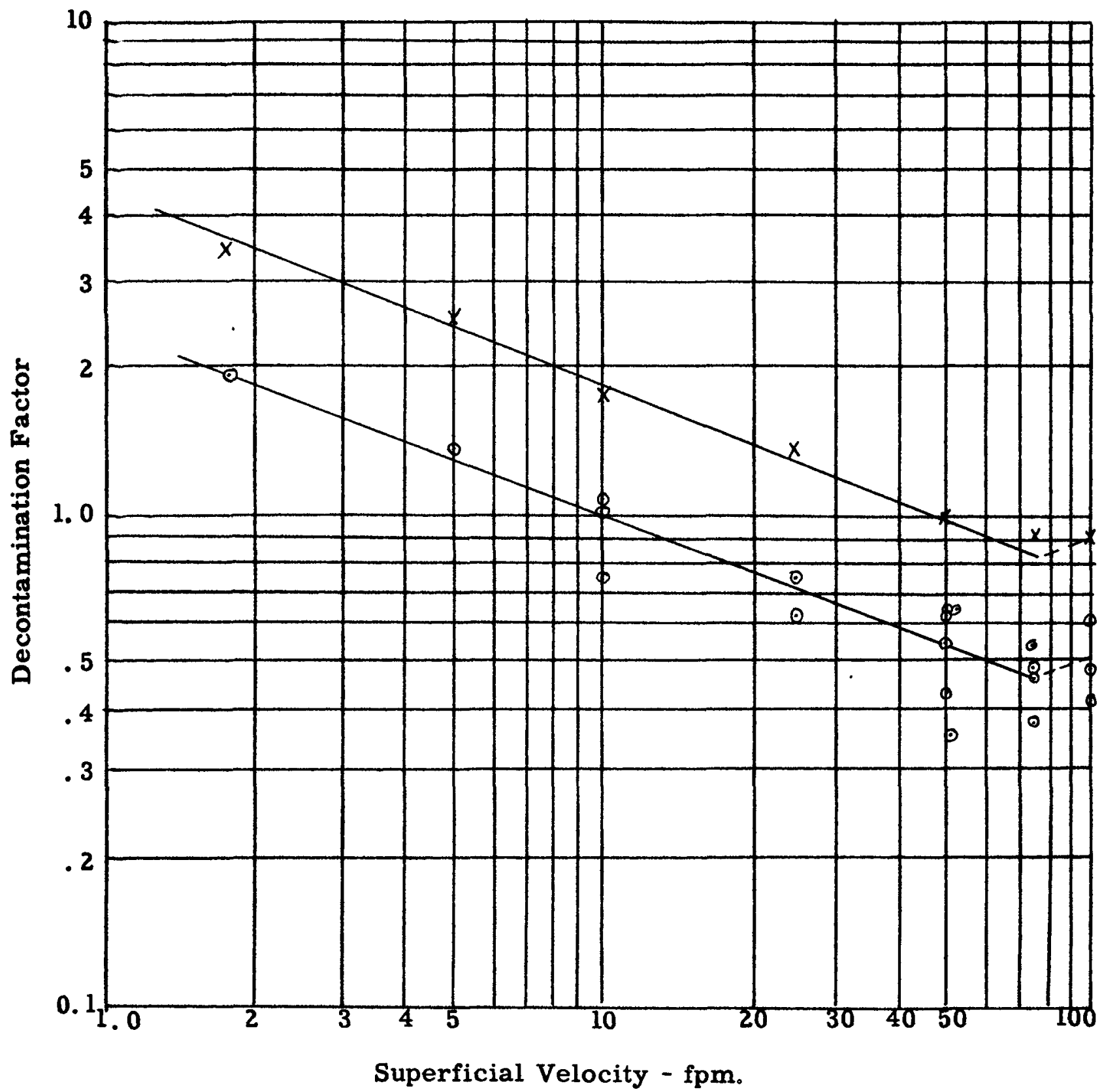


Figure C-5

GRAPH OF DECONTAMINATION FACTOR

VS. SUPERFICIAL VELOCITY

No. 55 Fiberglas, $\rho_{\mathrm{p}}=9.0$ pcf.

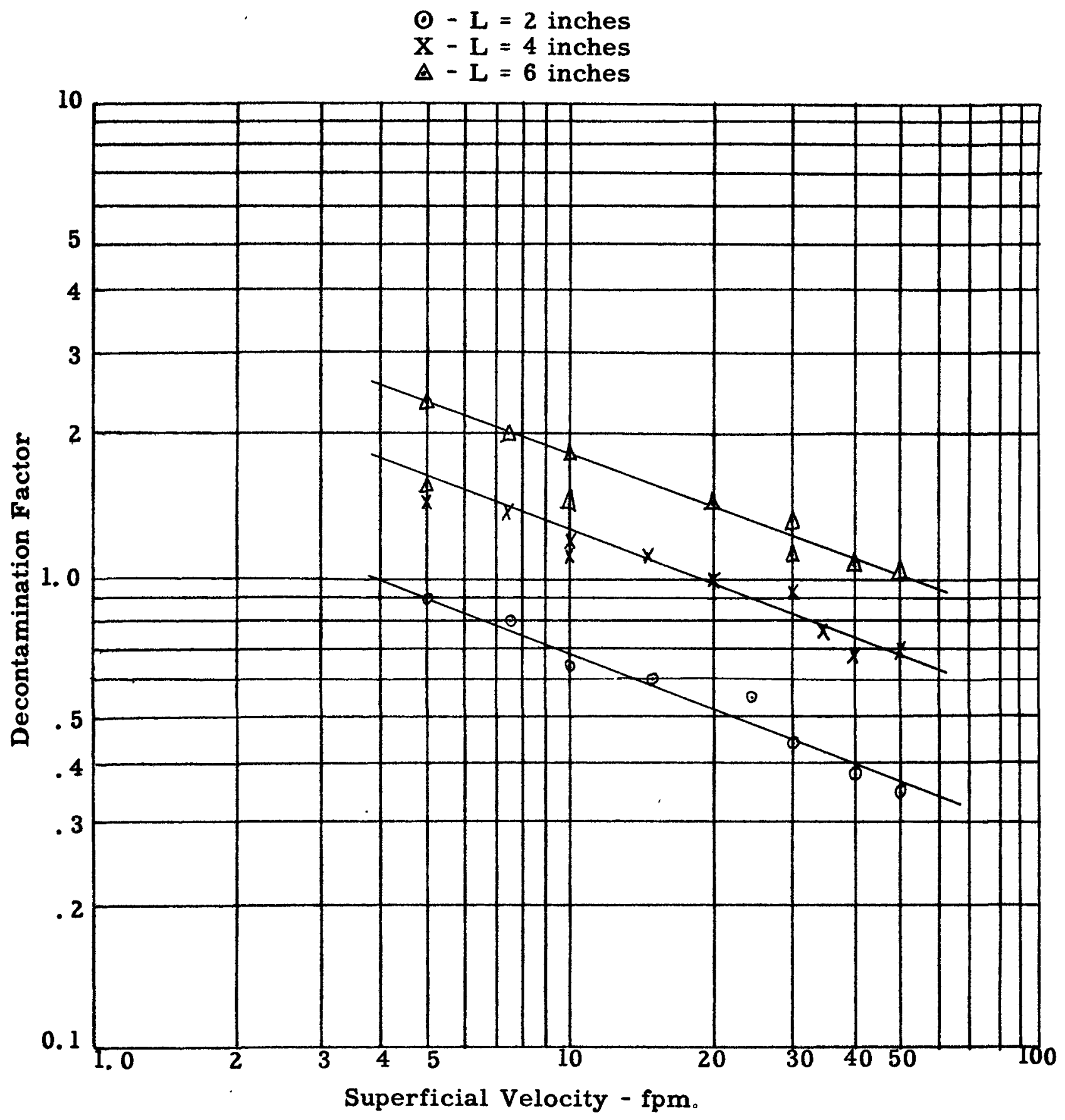


Figure C-6

CROSS-PLOT OF DECONTAMINATION FACTOR

VS. BED DEPTH

No. 55 Fiberglas

$\begin{array}{ll}\Delta-\rho_{\rho}=3.0 \text { pcf., } V=20 \mathrm{fpm} . & O-\rho_{p}=9.0 \text { pcf., } \mathrm{V}=5 \mathrm{fpm} . \\ \mathrm{G}-\rho_{\rho}=3.0 \mathrm{pcf}, \mathrm{V}=50 \mathrm{fpm} . & \nabla-\rho_{\rho}=9.0 \text { pcf., } \mathrm{V}=30 \mathrm{fpm} . \\ \mathrm{X}-\rho_{\rho}=6.0 \text { pcf., } \mathrm{V}=30 \mathrm{fpm} . & \end{array}$

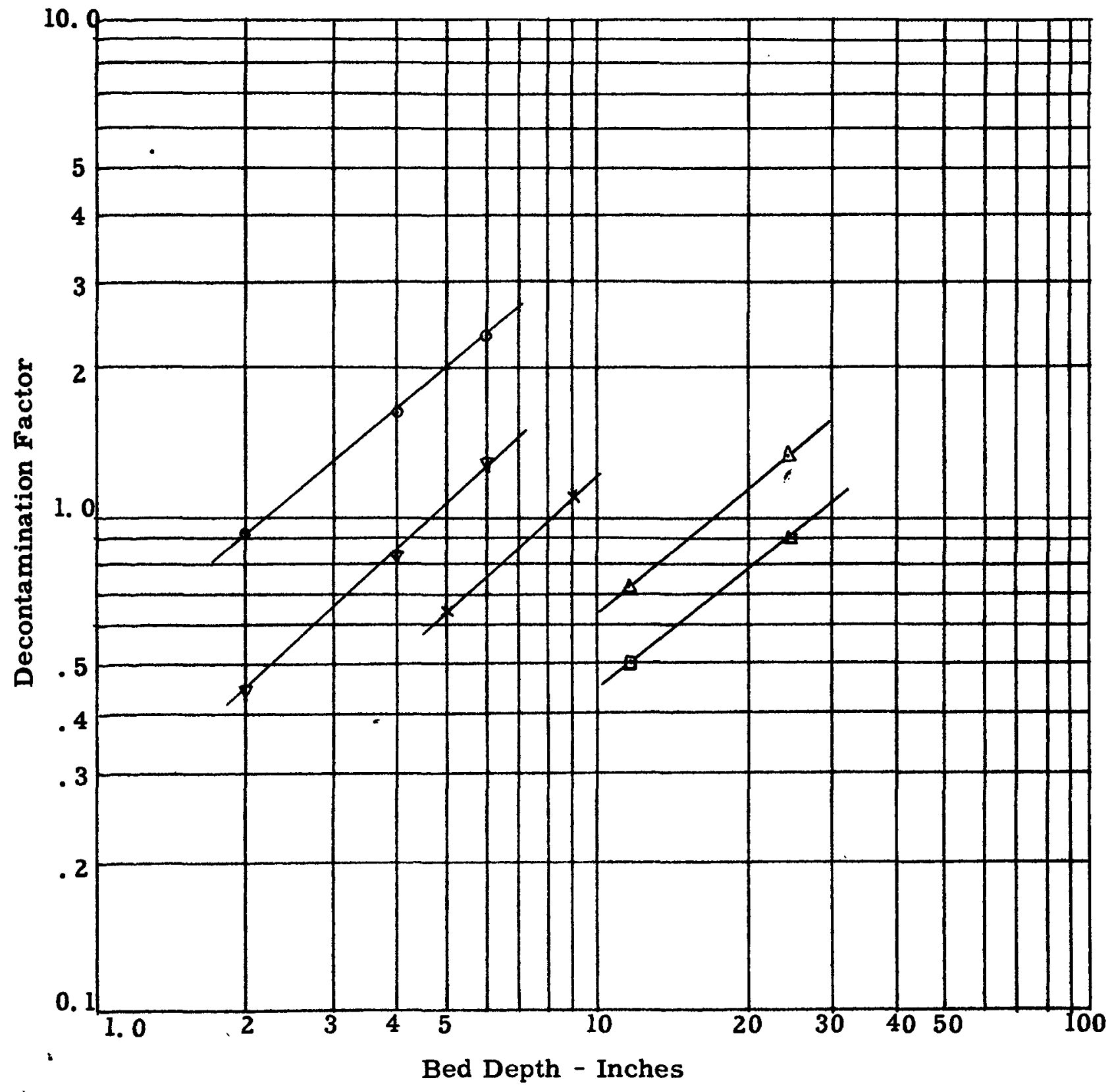




$$
-55-56
$$

Figure C-7

CROSS-PLOT OF DECONTAMINATION FACTOR VS. PACKING DENSITY

No. 55 Fiberglas

$$
\begin{aligned}
& 0-L=6 \text { inches, } V=10 \text { fpm. } \\
& X-L=6 \text { inches, } V=50 \mathrm{fpm} . \\
& \Delta-L=9 \text { inches, } V=10 \mathrm{fpm} . \\
& \square-L=9 \text { inches, } V=5 \mathrm{fpm} .
\end{aligned}
$$

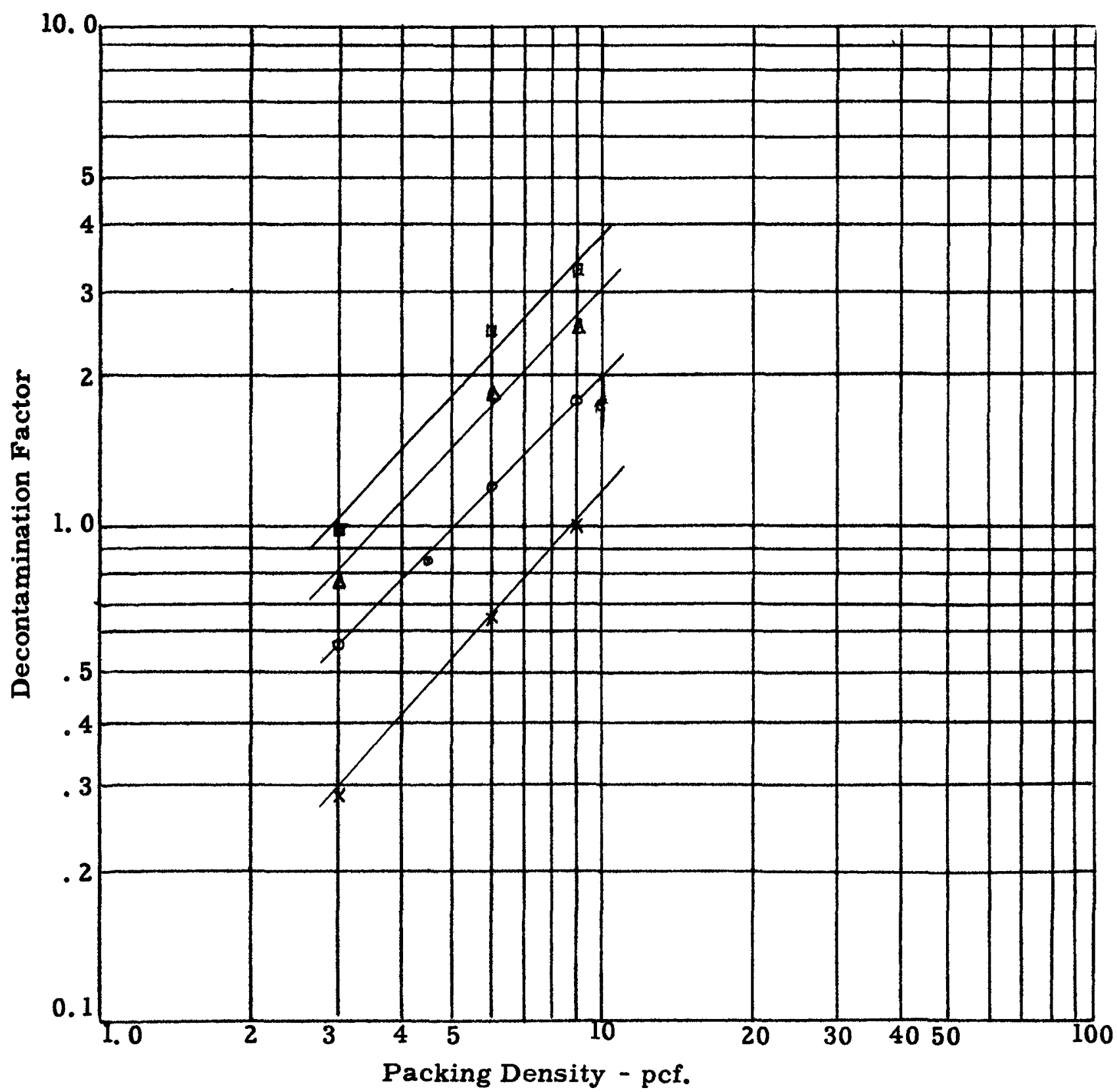


$-56-57$

HW- 20487

Figure C-8

GRAPH OF DECONTAMINATION FACTOR

VS. SUPERFICIAL VELOCITY

No. 55P Fiberglas, $\rho_{\mathrm{p}}=3.0$ pcf.

$0-L=6$ inches

$X-L=12$ inches

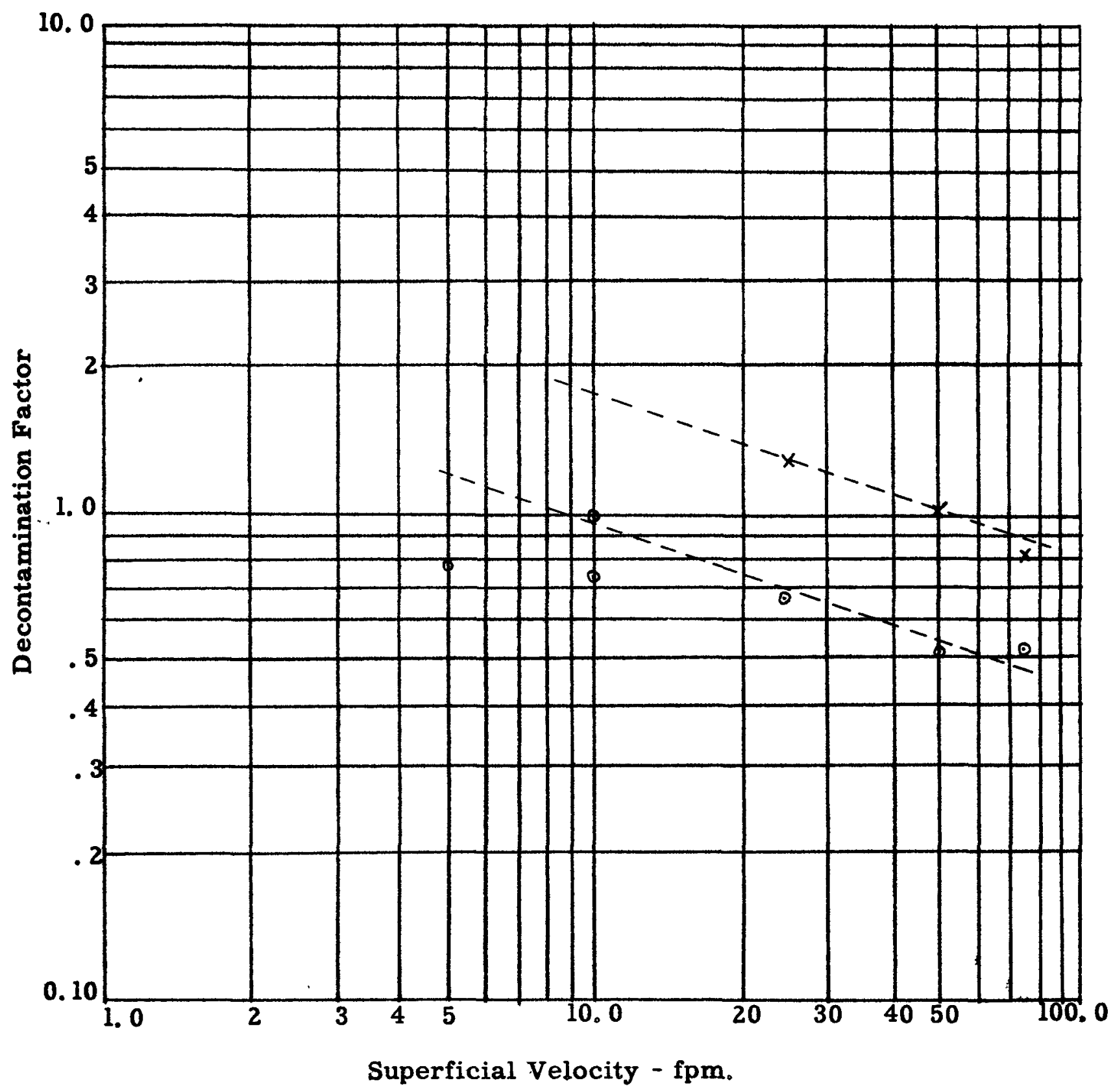


Figure C-9

GRAPH OF DECONTAMINATION FACTOR

VS. SUPERFICIAL VELOCITY

AA Fiberglas, $P_{p}=0.6$ pcf.

$$
\begin{aligned}
& \odot-L=0.5 \text { inch } \\
& X-L=1.0 \text { inch } \\
& \Delta-L=1.5 \text { inches }
\end{aligned}
$$

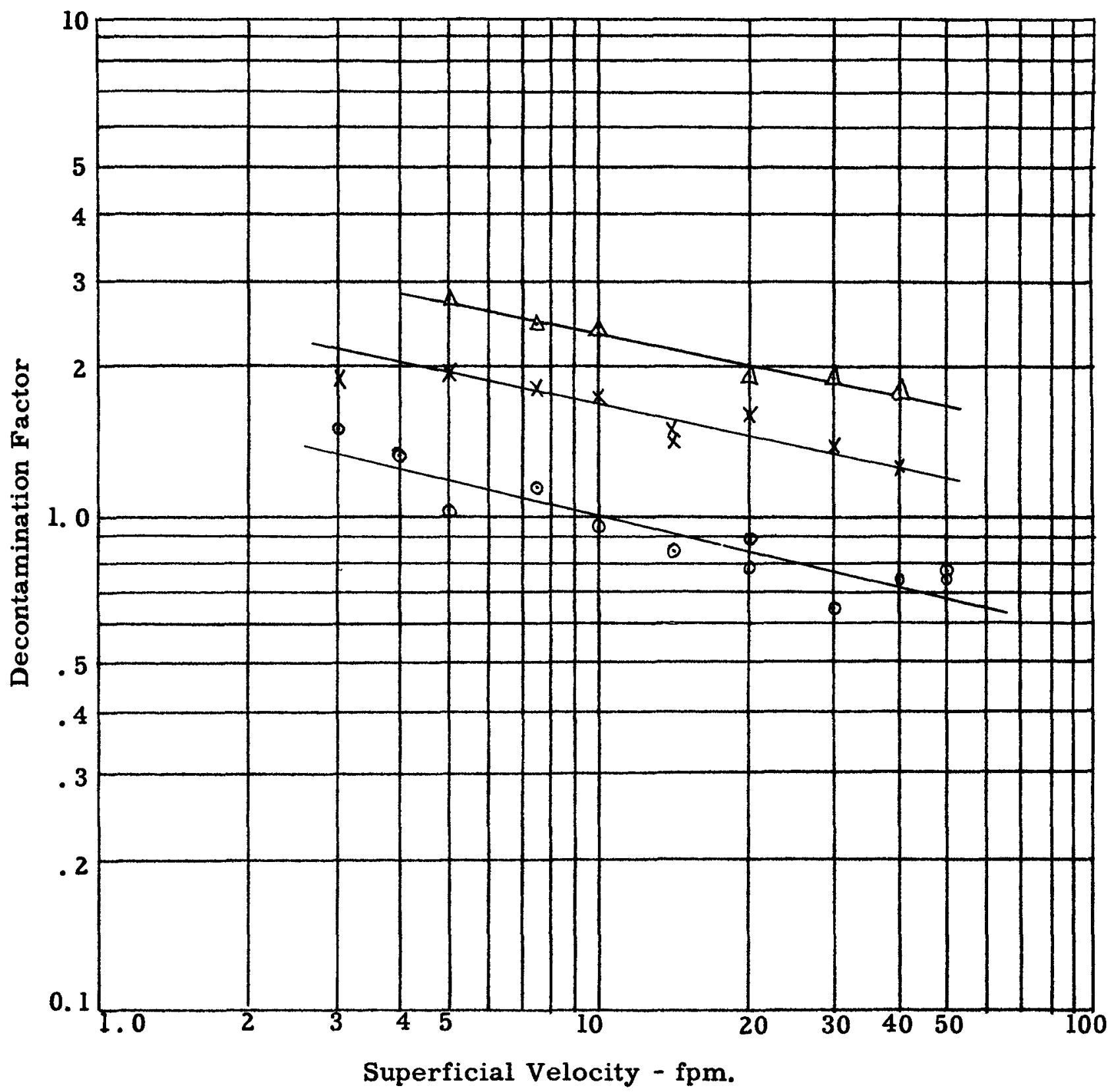




$$
-58-\quad 59
$$

Figure C-10

GRAPH OF DECONTAMINATION FACTOR

VS. SUPERFICIAL VELOCITY

AA FIBERGLAS

$$
\begin{aligned}
& \Delta-\rho_{p}=1.2 \text { pcf.; } L=0.25 \text { inch } \\
& X-\rho_{p}=1.2 \text { pcf.; } L=0.50 \text { inch } \\
& \circlearrowleft-\rho_{p}=1.8 \text { pcf.; } L=0.50 \text { inch }
\end{aligned}
$$

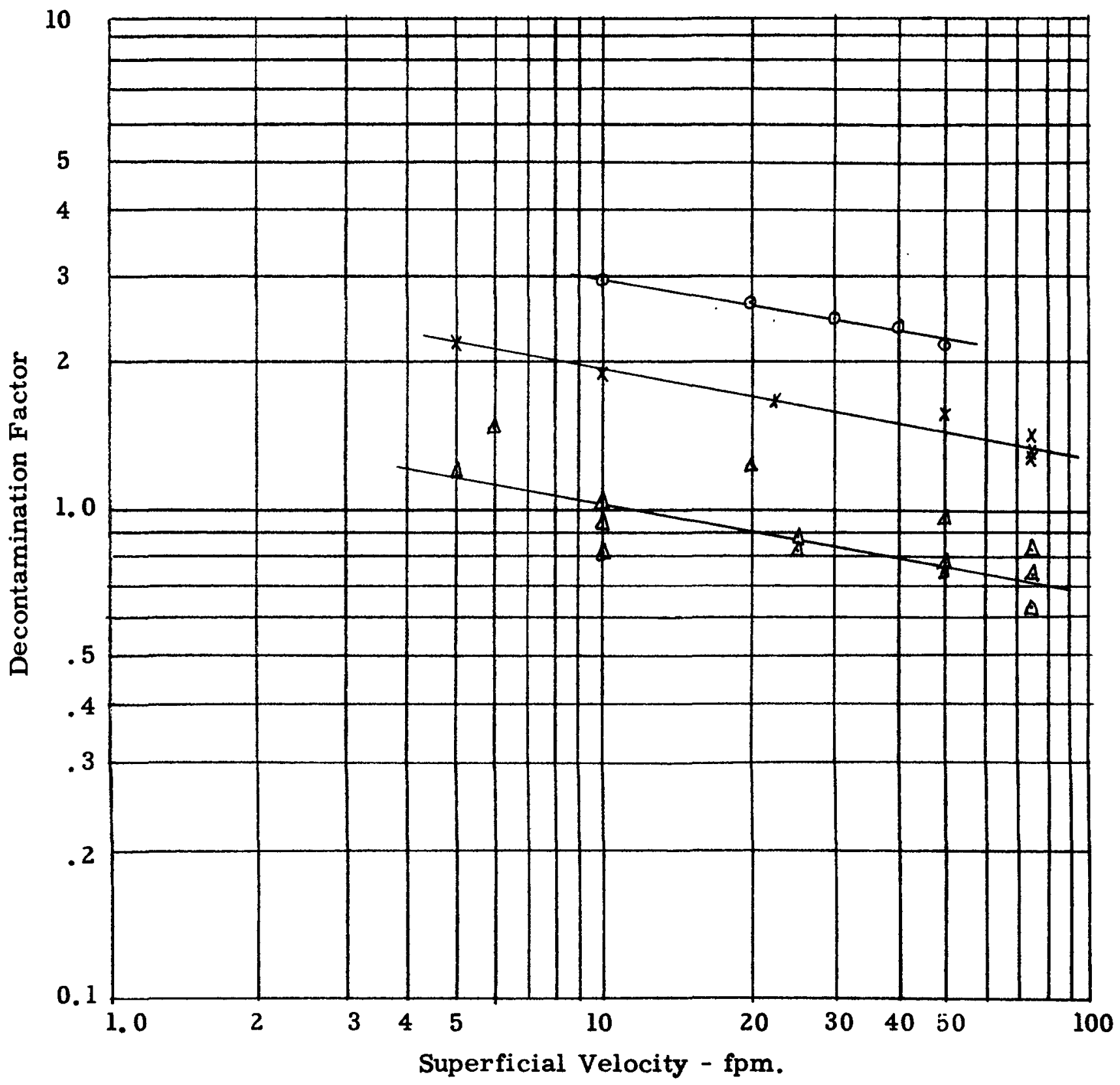




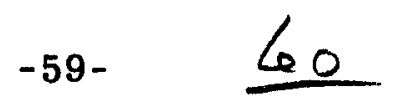

Figure C-11

CROSS-PLOT OF DECONTAMINATION FACTOR -

VS. BED DEPTH

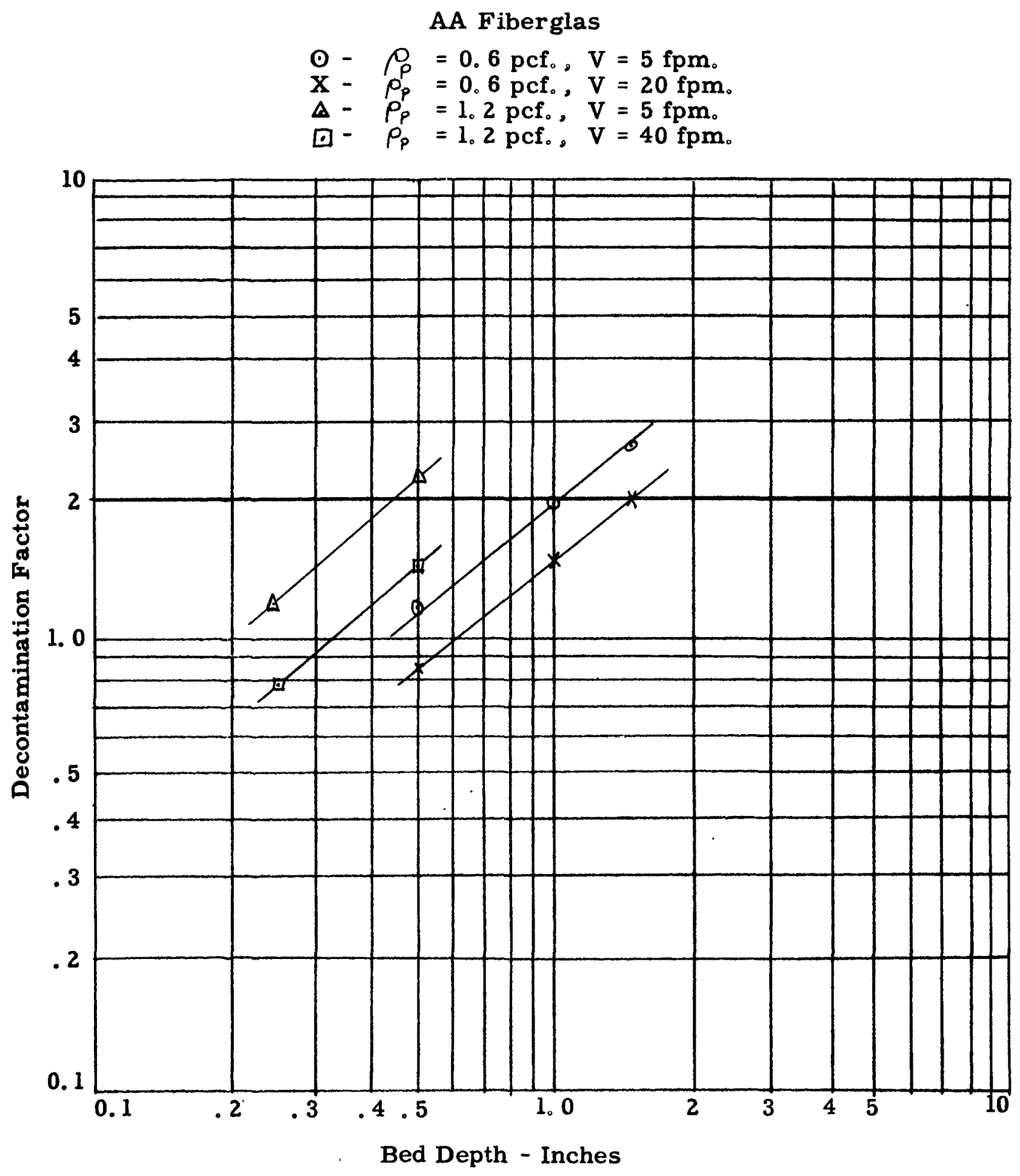




$$
-60-61
$$

Figure $\mathrm{C}-12$

CROSS-PLOT OF DECONTAMINATION

FACTOR VS。PACKING DENSITY

$$
\begin{aligned}
& \text { AA FIBERGLAS } \\
& \hline L=0.5 \text { inch } \\
& \odot-L=10 \mathrm{fpm} . \\
& X-L=20 \mathrm{fpm} . \\
& \Delta-L=40 \mathrm{fpm} .
\end{aligned}
$$

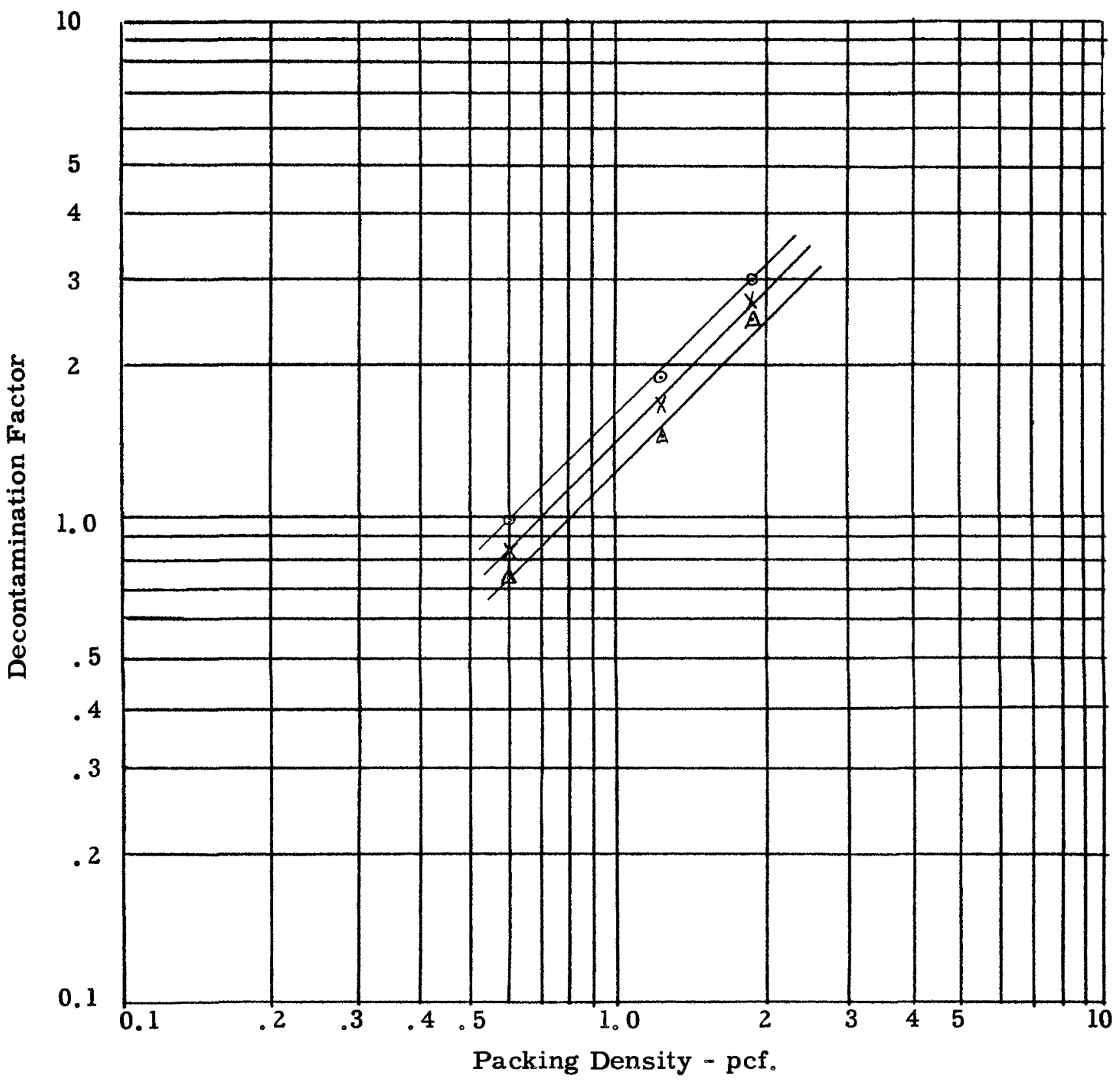




$$
-61-\quad 62
$$

Figure C-13

GRAPH OF DECONTAMINA TION FACTOR

VS. SUPERFICIAL VELOCITY

$B$ ( $L$ and $R$ ) Fiberglas, $\rho_{p}=4.0$ pcf.

$$
\begin{aligned}
& X-L=0.50 \text { inch } \\
& \mathbb{D}-L=0.25 \text { inch }
\end{aligned}
$$

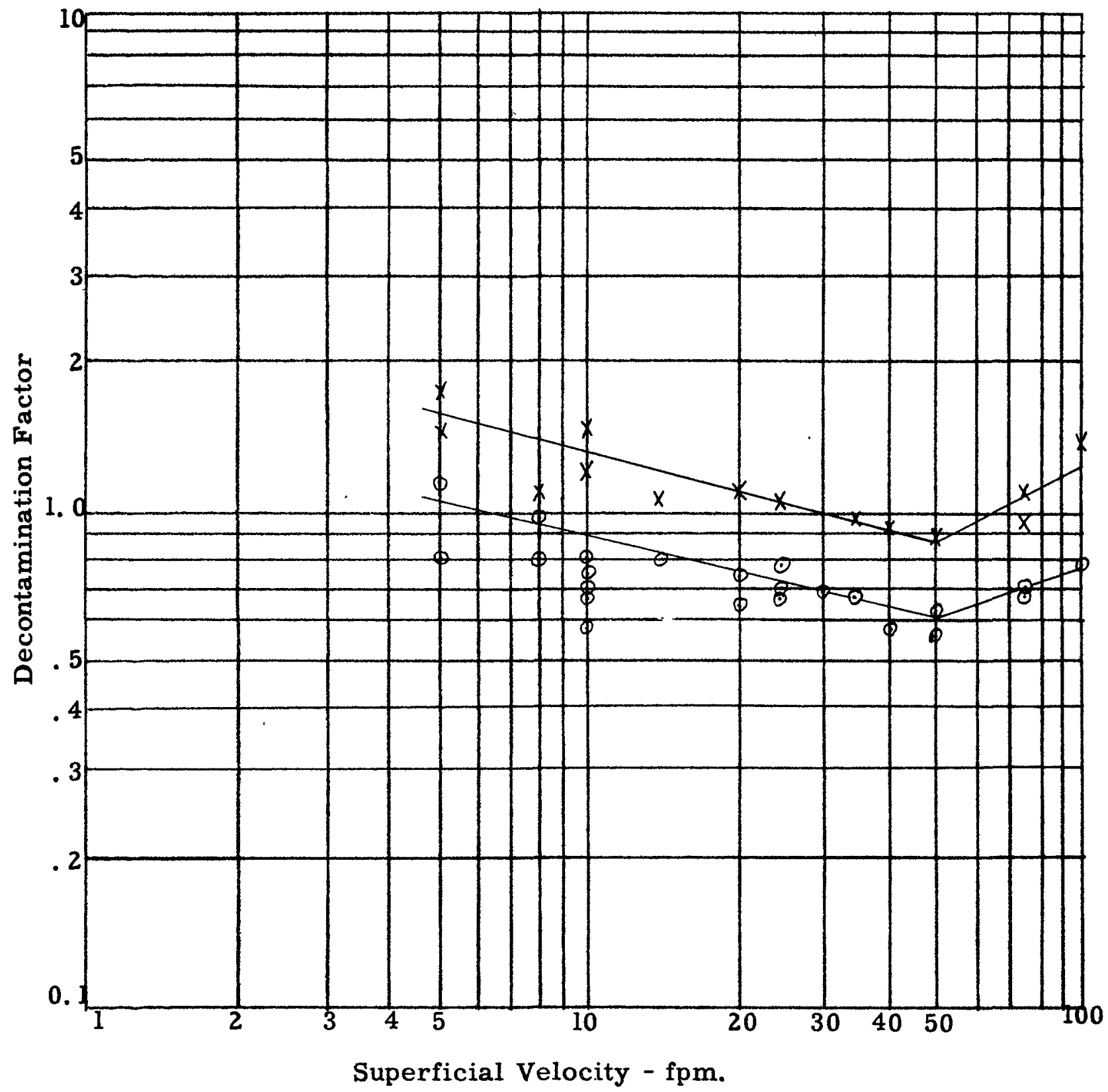


Figure C-14

GRAPH OF DECONTAMINATION FACTOR

VS. SUPERFICIAL VELOCITY

B Fiberglas, $\overbrace{p}=0.7$ pcf。

$L_{1}=1.0$ inch

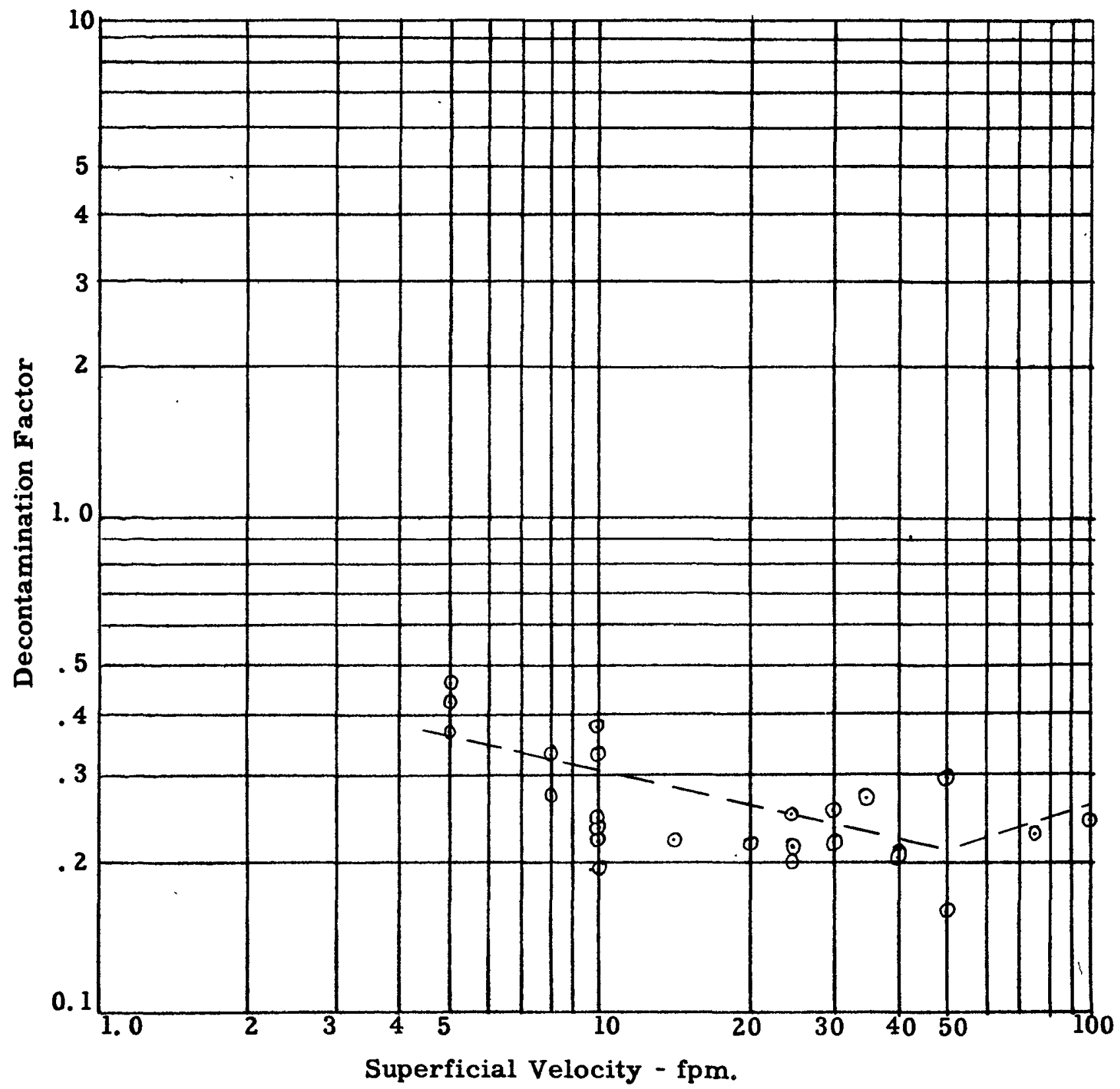


$-63-\quad 64$

Figure C-15

GRAPH OF DECONTAMINATION FACTOR

VS. SUPERFICIAL VELOCITY

No. 450 Fiberglas, $P_{p}=5.7$ pcf.

$\mathrm{L}=8$ inches

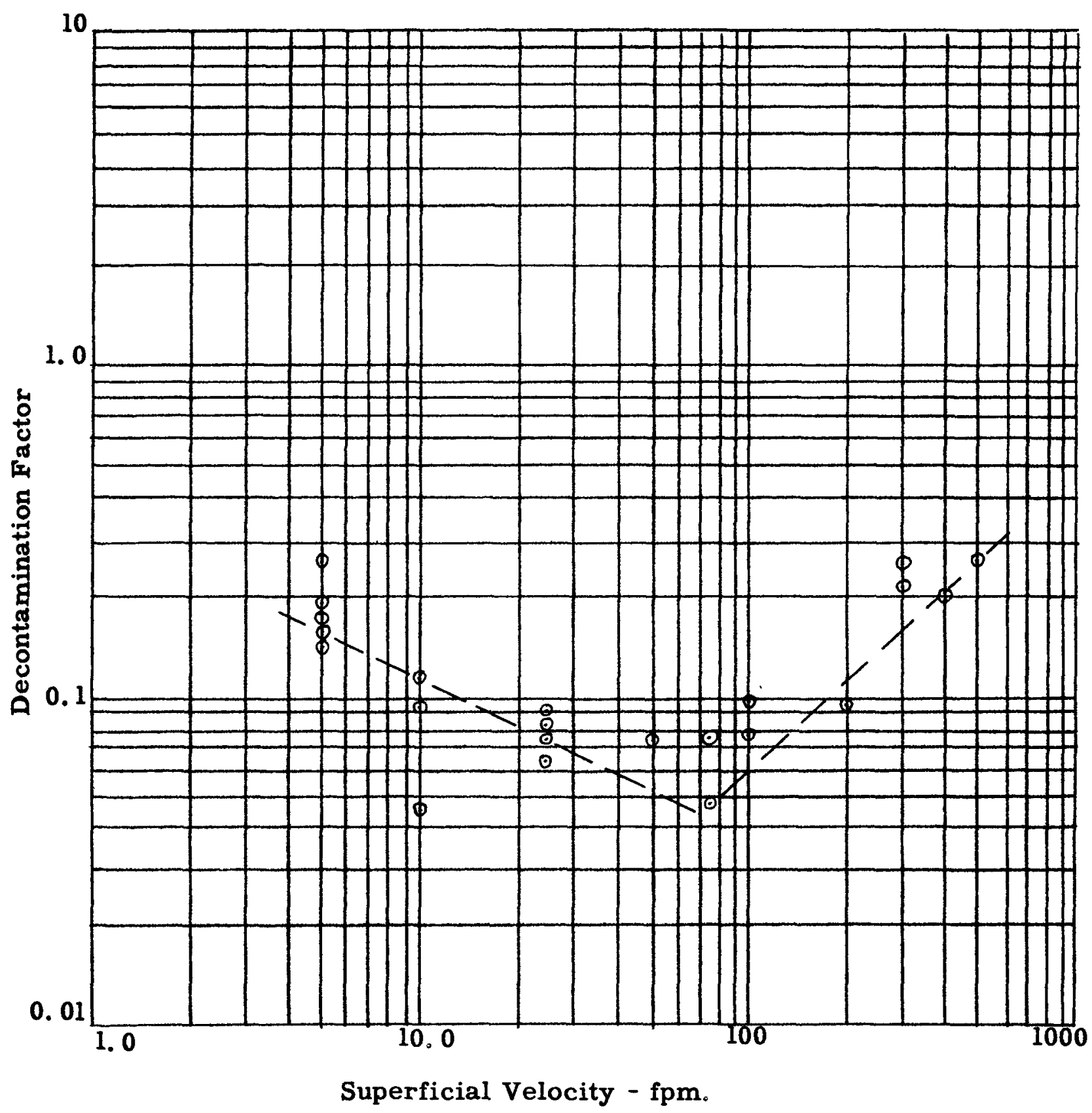


Figure C-16

GRAPH OF DECONTAMINA TION FACTOR

VS. SUPERFICIAL VELOCITY

No. 600 Fiberglas, $P_{p}=5.1$ pcf.

$L=7$ inches

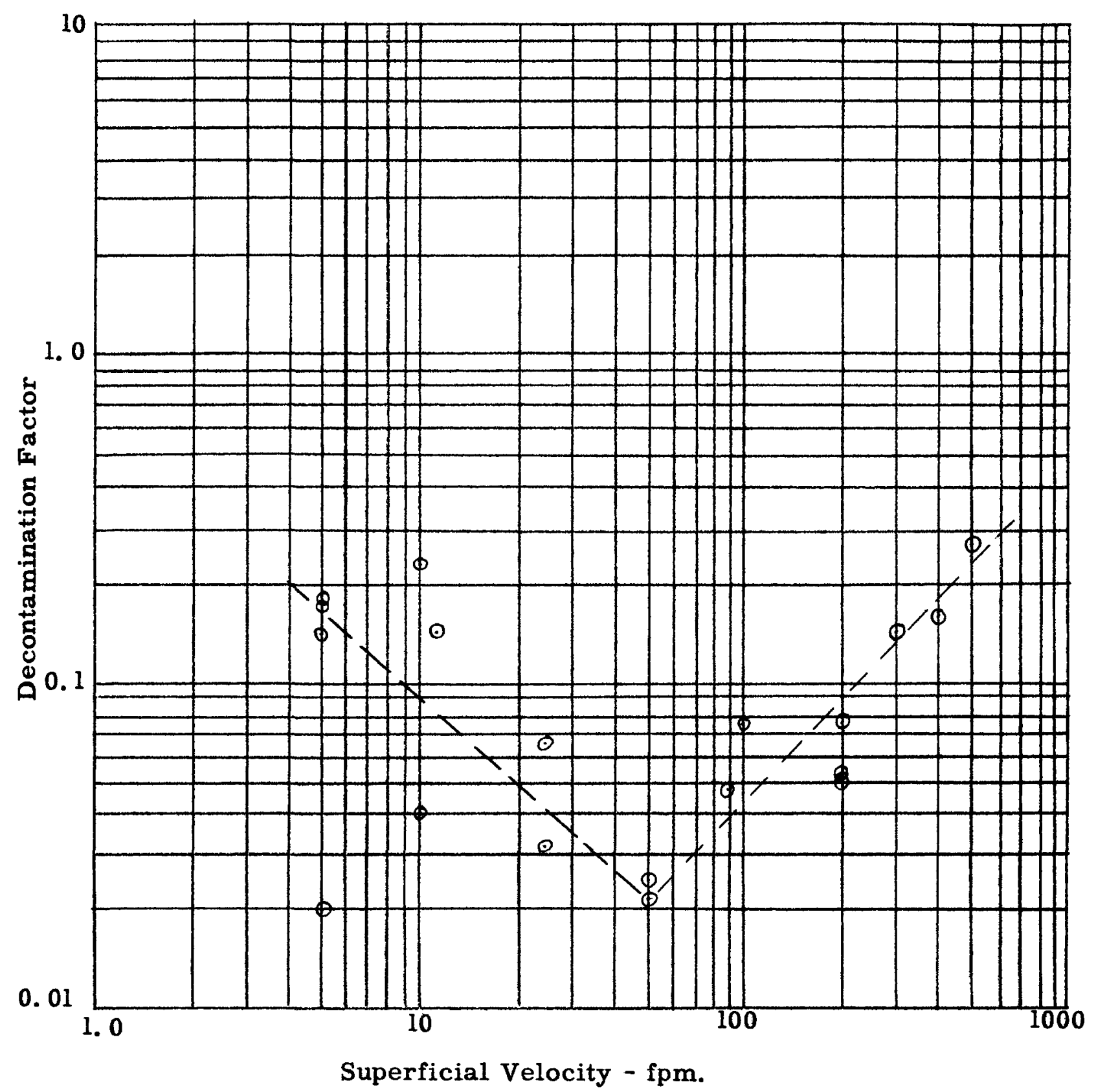


$-65-\quad 66$

Figure C-17

GRAPH OF DECONTAMINATION FACTOR

VS. SUPERFICIAL VELOCITY

No. 800 Fiberglas, $\rho_{p}=6.6$ pcf.

$\mathrm{L}=8$ inches

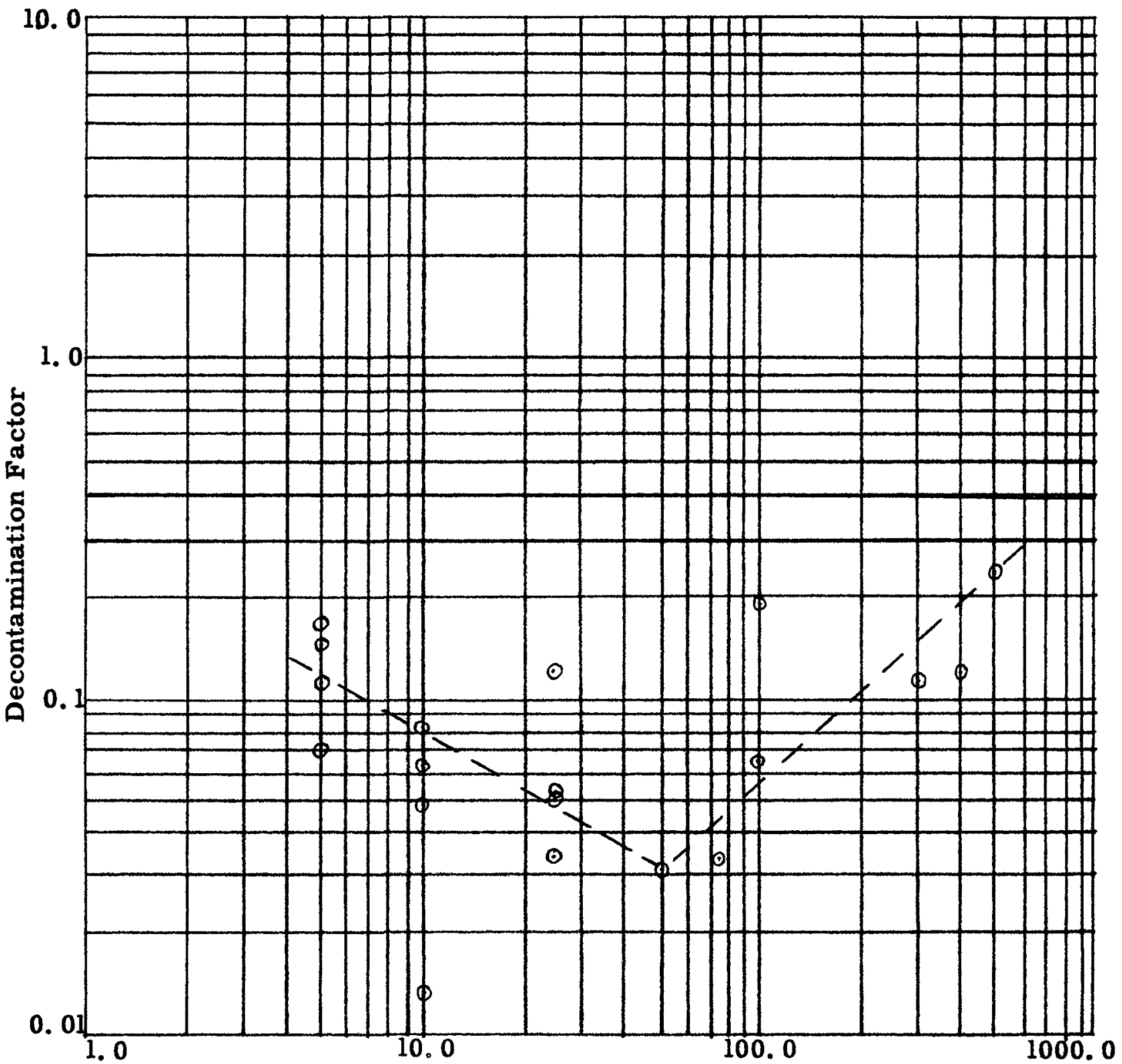

Superficial Velocity - fpm. 


$$
-66-\quad 67
$$

Figure C-18

GRAPH OF DECONTAMINATION FACTOR

VS. SUPERFICIAL VELOCITY

No. 115 Fiber, $\quad \rho_{p}=3.8$ pcf.

0 - $\mathrm{L}=10$ inches

$\Delta-L=20$ inches

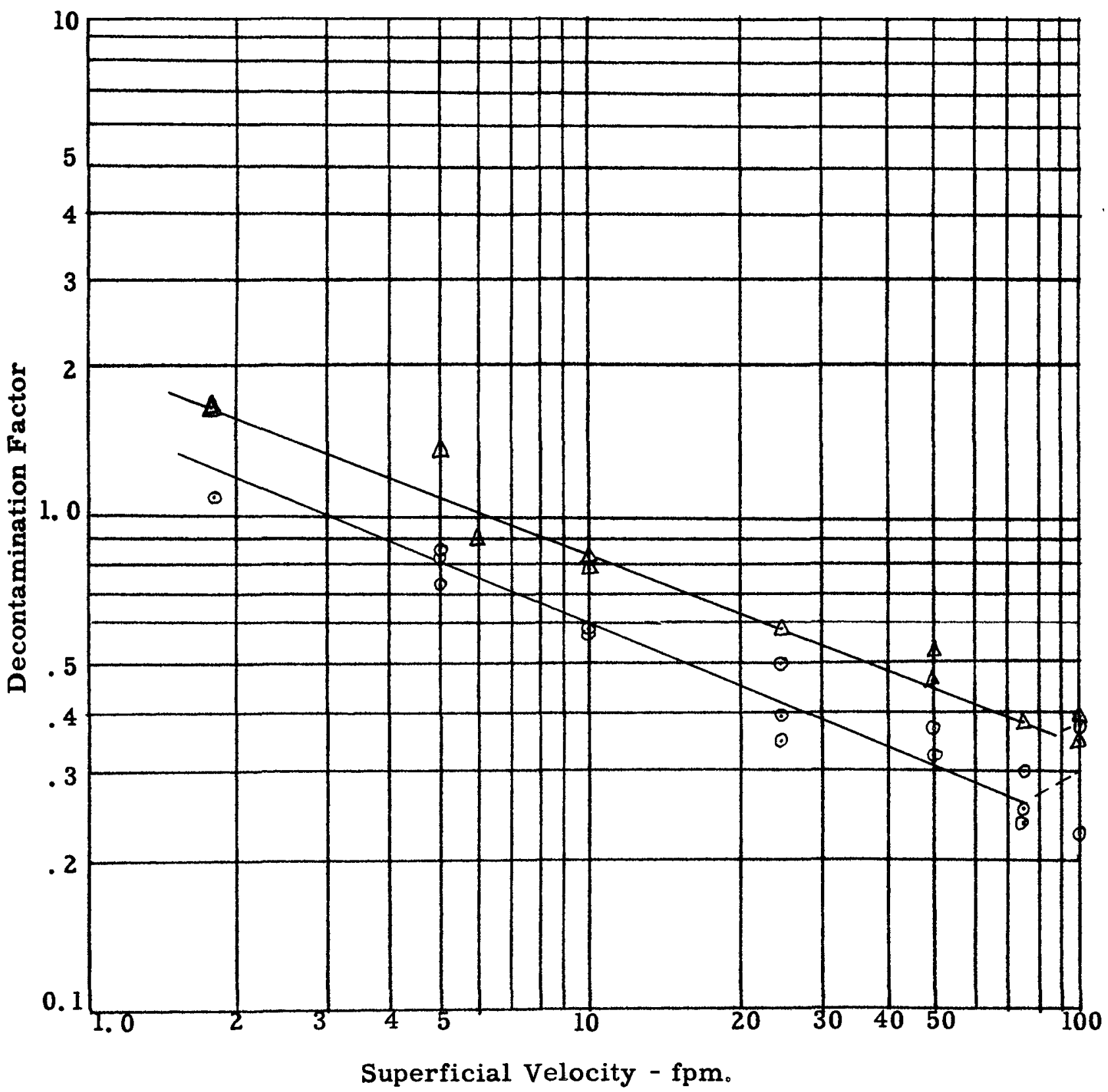


$-67-68$

Figure C-19

CROSS PLOT OF DECONTAMINATION FACTOR

VS. BED DEPTH

No. 115 Fiber $\quad \rho_{p}=3.8$ pcf.

$X-V=2 \mathrm{fpm}$.
$0-V=10 \mathrm{fpm}$.
$\Delta-V=25 \mathrm{fpm}$.
$\square-V=50 \mathrm{fpm}$.

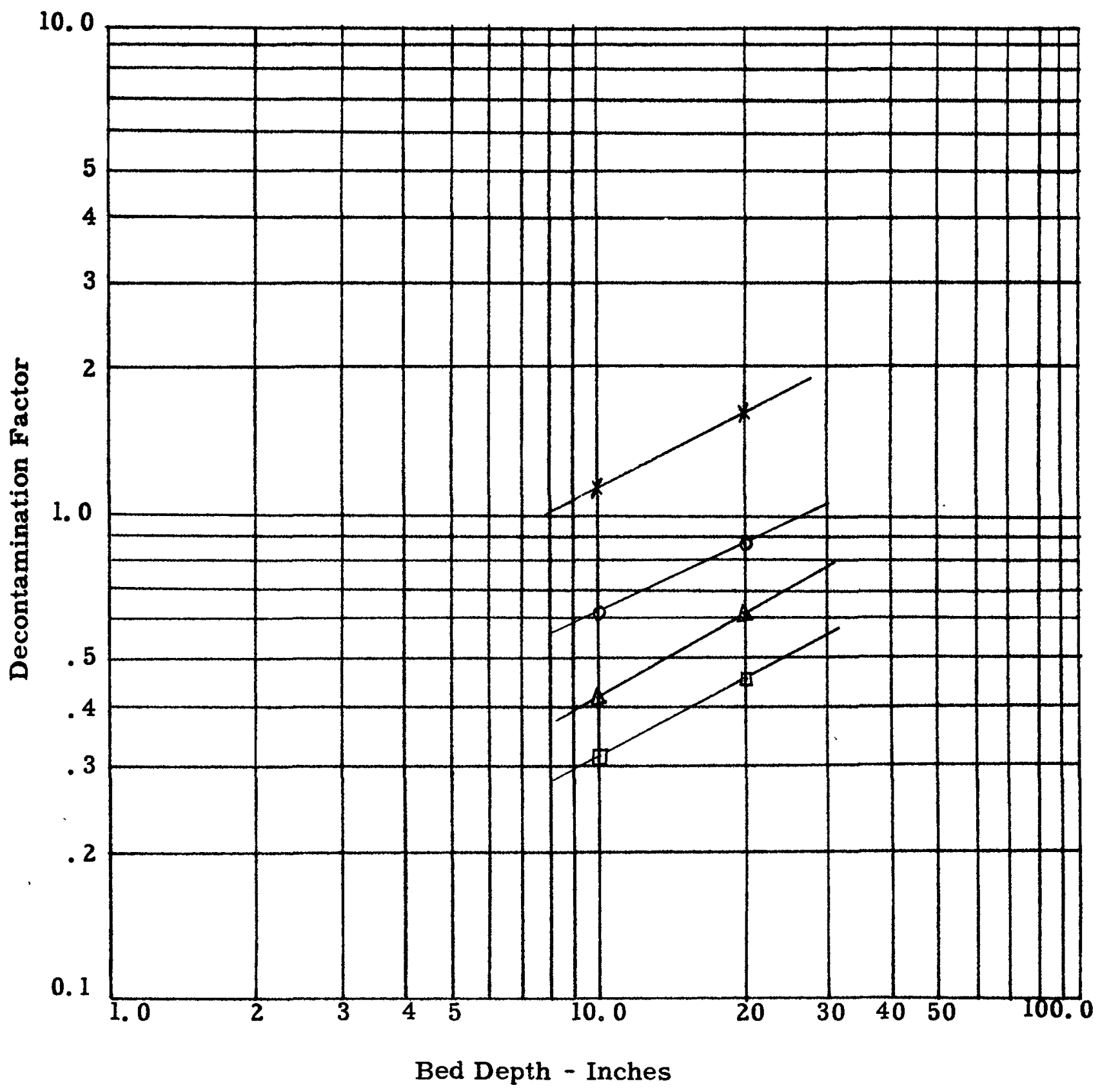


$-68-\quad 69$

Figure C-20

GRAPH OF DECONTAMINA TION FACTOR VS. SUPERFICIAL VELOCITY

No. $115 \mathrm{~K}$ Fiberglas, $P_{\mathrm{p}}=1.5$ pcf.

$0-\mathrm{L}=6$ inches

$X-L=12$ inches

$\Delta-\mathrm{L}=18$ inches

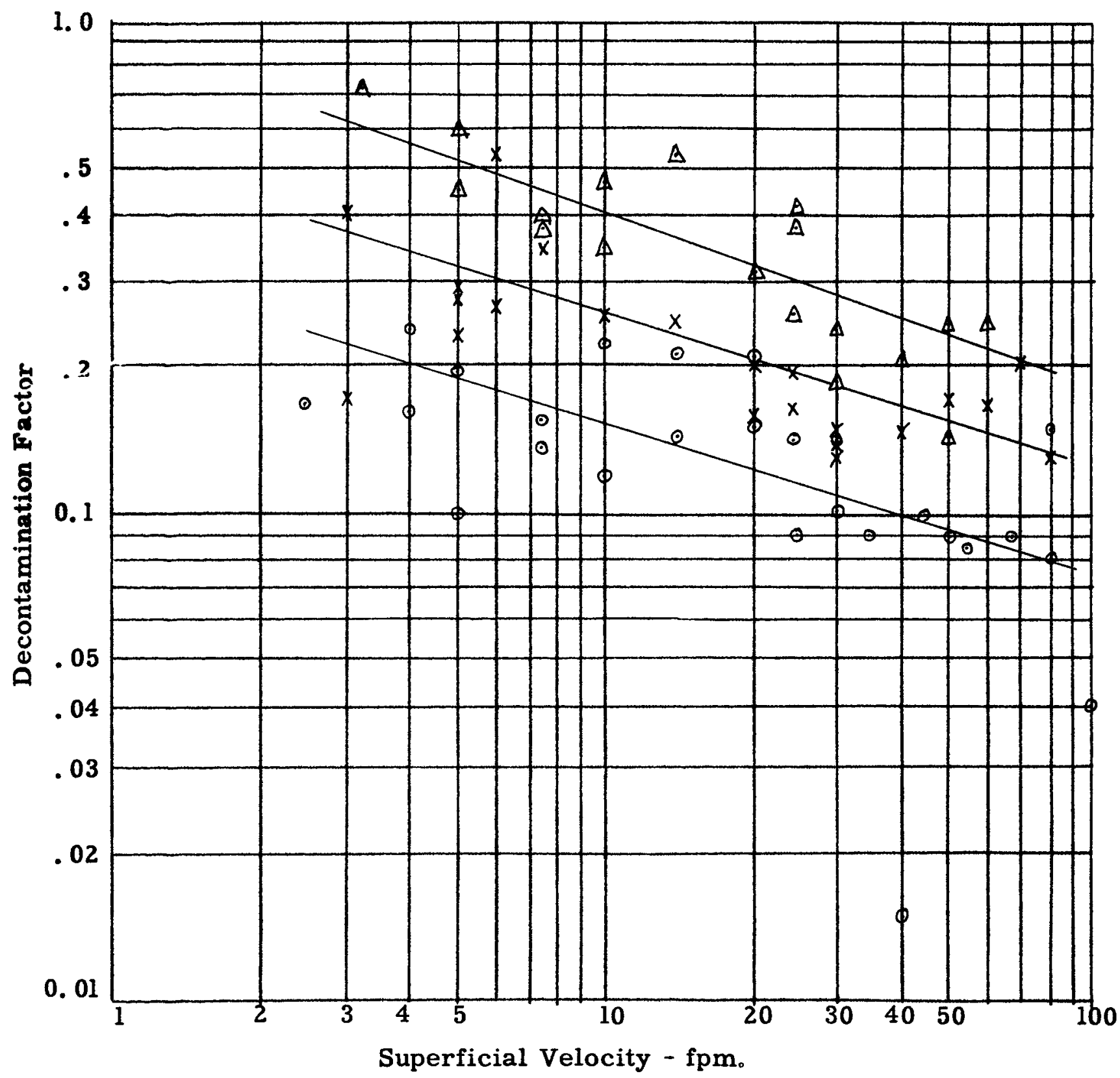




$$
-69-\quad 70
$$

Figure C-21

GRAPH OF DECONTAMINATION FACTOR

VS. SUPERFICIAL VELOCITY

No. $115 \mathrm{~K}$ Fiberglas, $\rho_{\mathrm{p}}=3.0$ pcf.

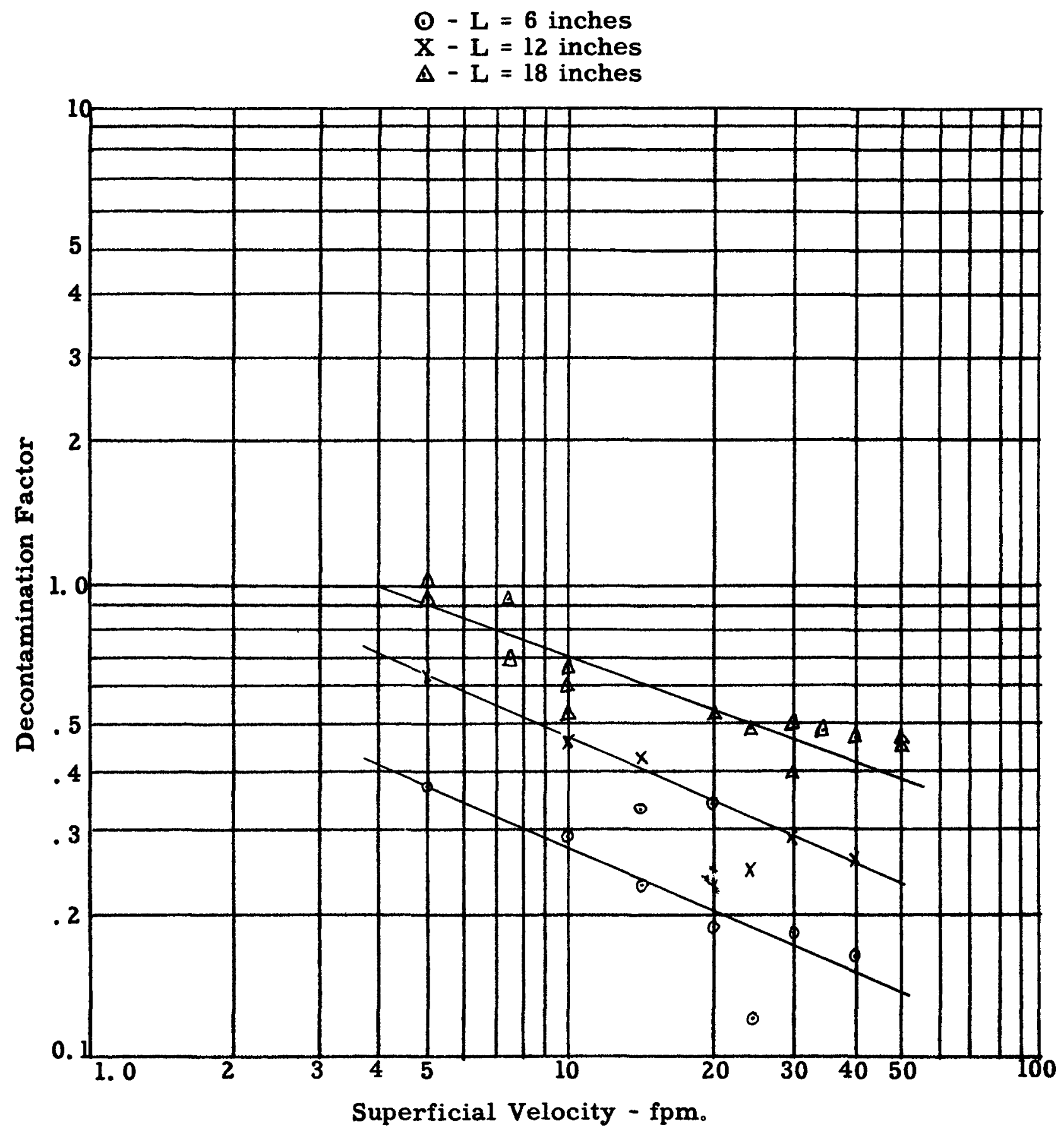




\section{Figure C-22}

GRAPH OF DECONTAMINATION FACTOR

VS. SUPERFICIAL VELOCITY

No. $115 \mathrm{~K}$ Fiberglas, $P_{\mathrm{p}}=6.0$ pcf.

$$
\begin{aligned}
& O-L=6 \text { inches } \\
& X-L=12 \text { inches } \\
& \Delta-L=18 \text { inches }
\end{aligned}
$$

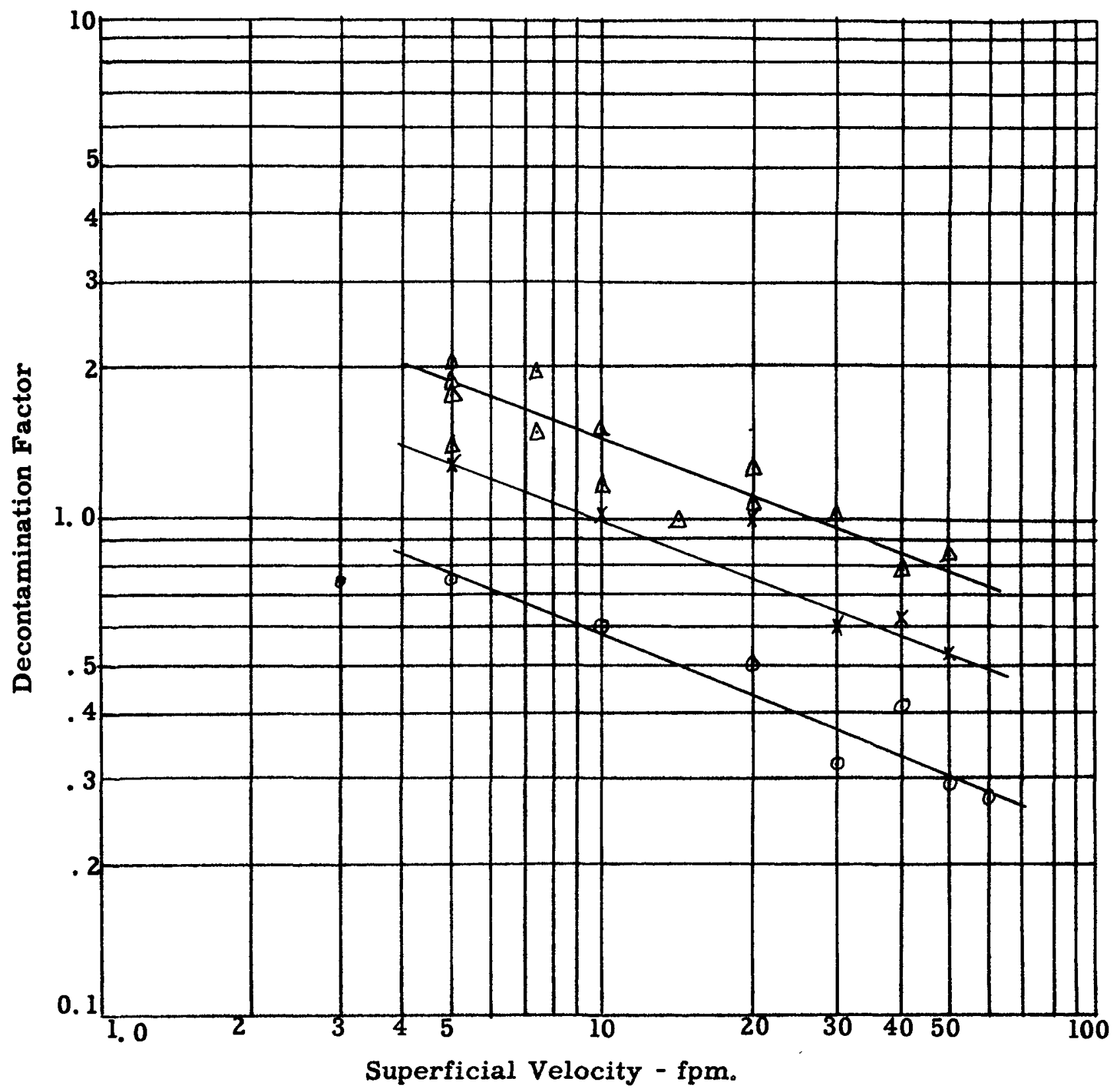




$$
-71-72
$$

Figure C-23

GRAPH OF DECONTAMINATION FACTOR

VS. SUPERFICIAL VELOCITY

No. 115K Fiberglas, $P_{\mathrm{p}}=9.0$ pcf.

$$
\begin{aligned}
& \text { Q }-\mathrm{L}=6 \text { inches } \\
& \mathrm{X}-\mathrm{L}=12 \text { inches }
\end{aligned}
$$

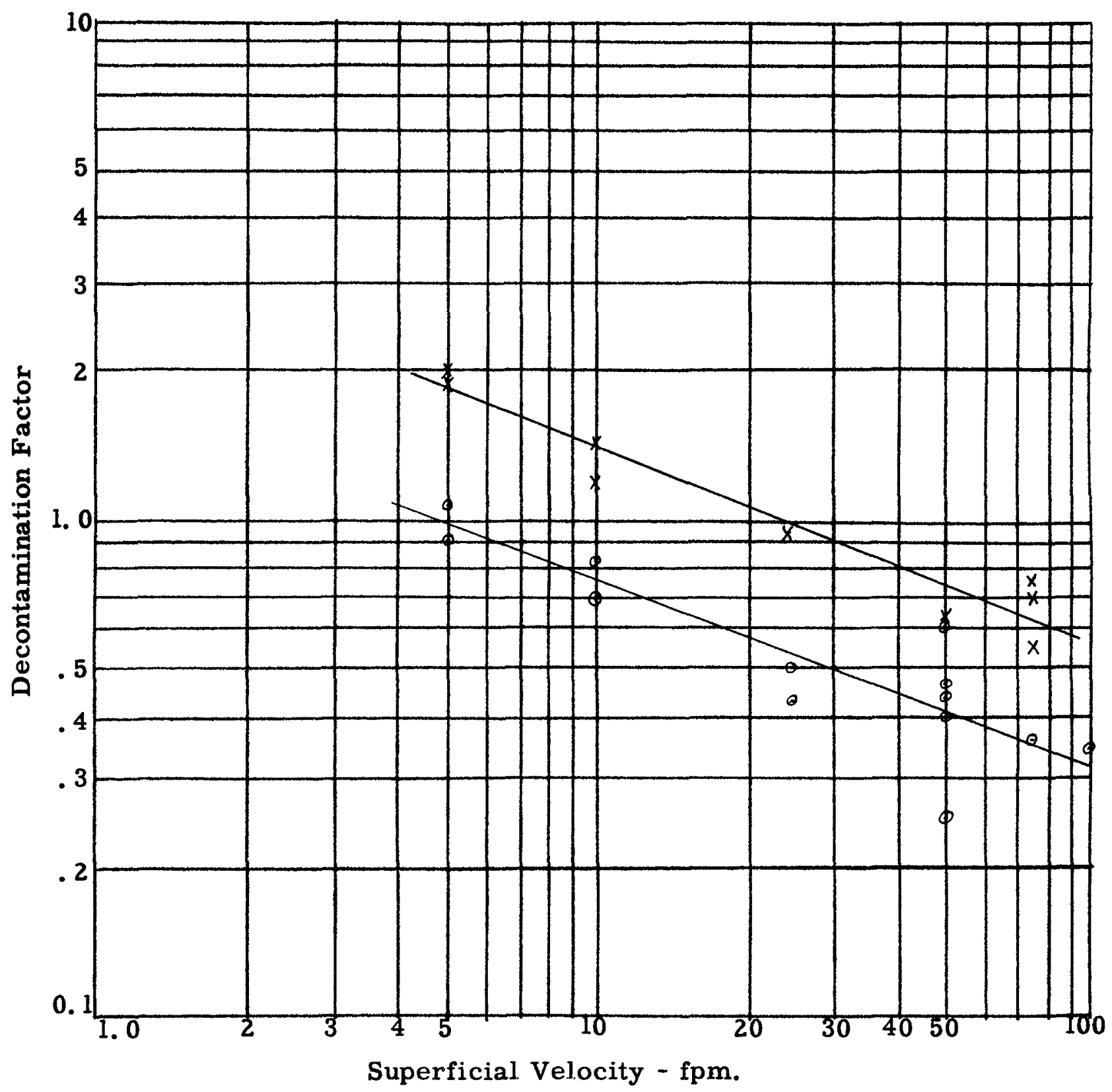




$$
-72-73
$$

Figure C-24

CROSS-PLOT OF DECONTAMINATION FACTOR

VS. BED DEPTH

No. 115K Fiberglas

$X-P_{p}=1.5$ pcf., $V=15$ fpm. $\square-\rho_{p}=6.0$ pcf.,$V=50 \mathrm{fpm}$.

$0-\rho_{p}=3.0$ pcf., $V=25$ fpm。 $\nabla-\rho_{P}=9.0$ pcf., $V=5$ fpm.

$\Delta-P_{p}=6.0$ pcf., $V=15$ fpm。

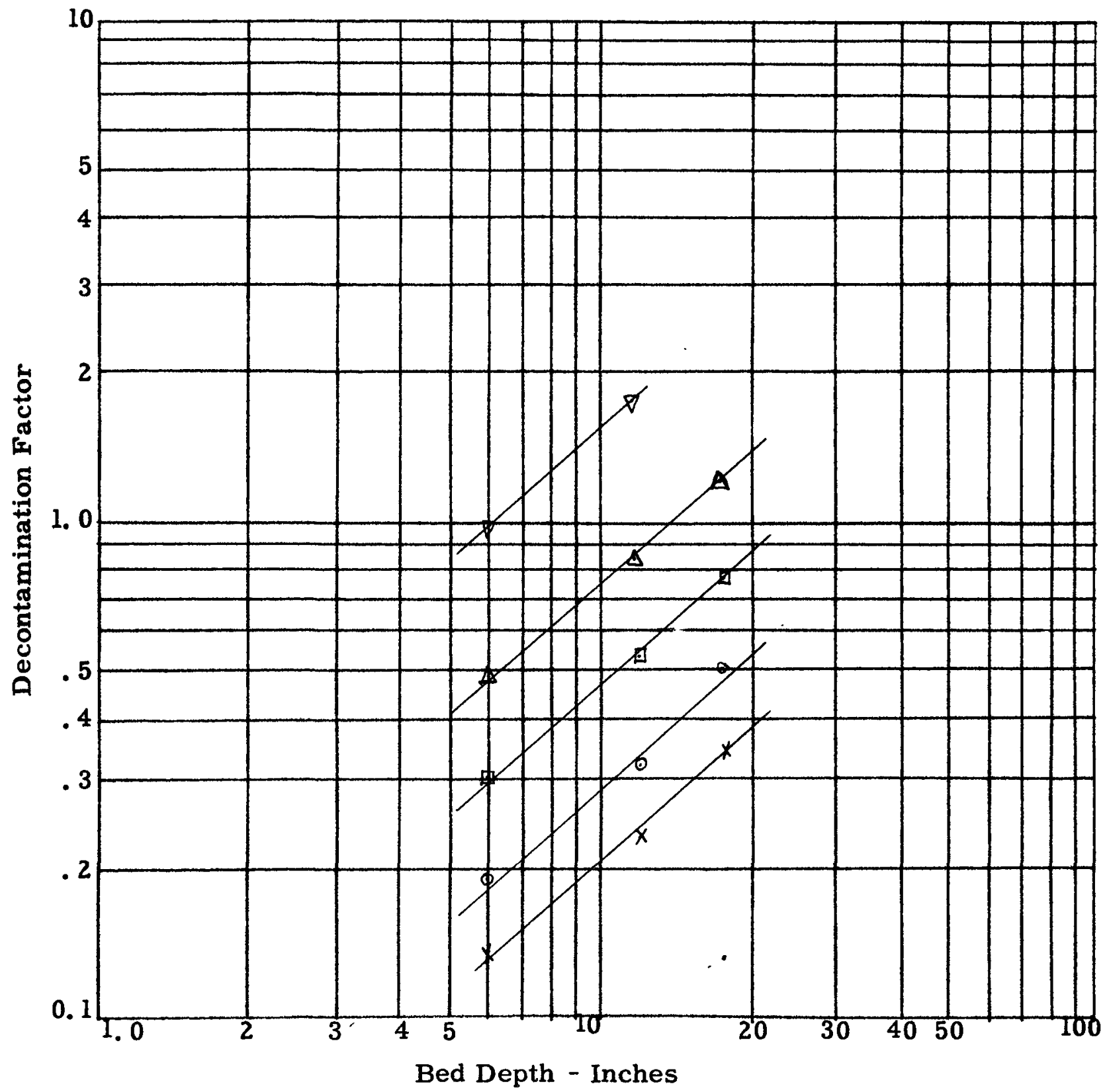




$$
-73->4
$$

Figure C-25

CROSS-PLOT OF DECONTAMINA TION FACTOR

VS. PACKING DENSITY

No. 115K Fiberglas
$X-L=6$ inches, $V=15$ fpm.
$\Delta-L=6$ inches, $V=30 \mathrm{fpm}$.
$\otimes-\mathrm{L}=12$ inches, $V=15 \mathrm{fpm}$.
G $-\mathrm{L}=12$ inches, $V=5 \mathrm{fpm}$.
$\odot-\mathrm{L}=12$ inches, $V=40 \mathrm{fpm}$.
$\nabla-\mathrm{L}=18$ inches, $V=5 \mathrm{fpm}$.

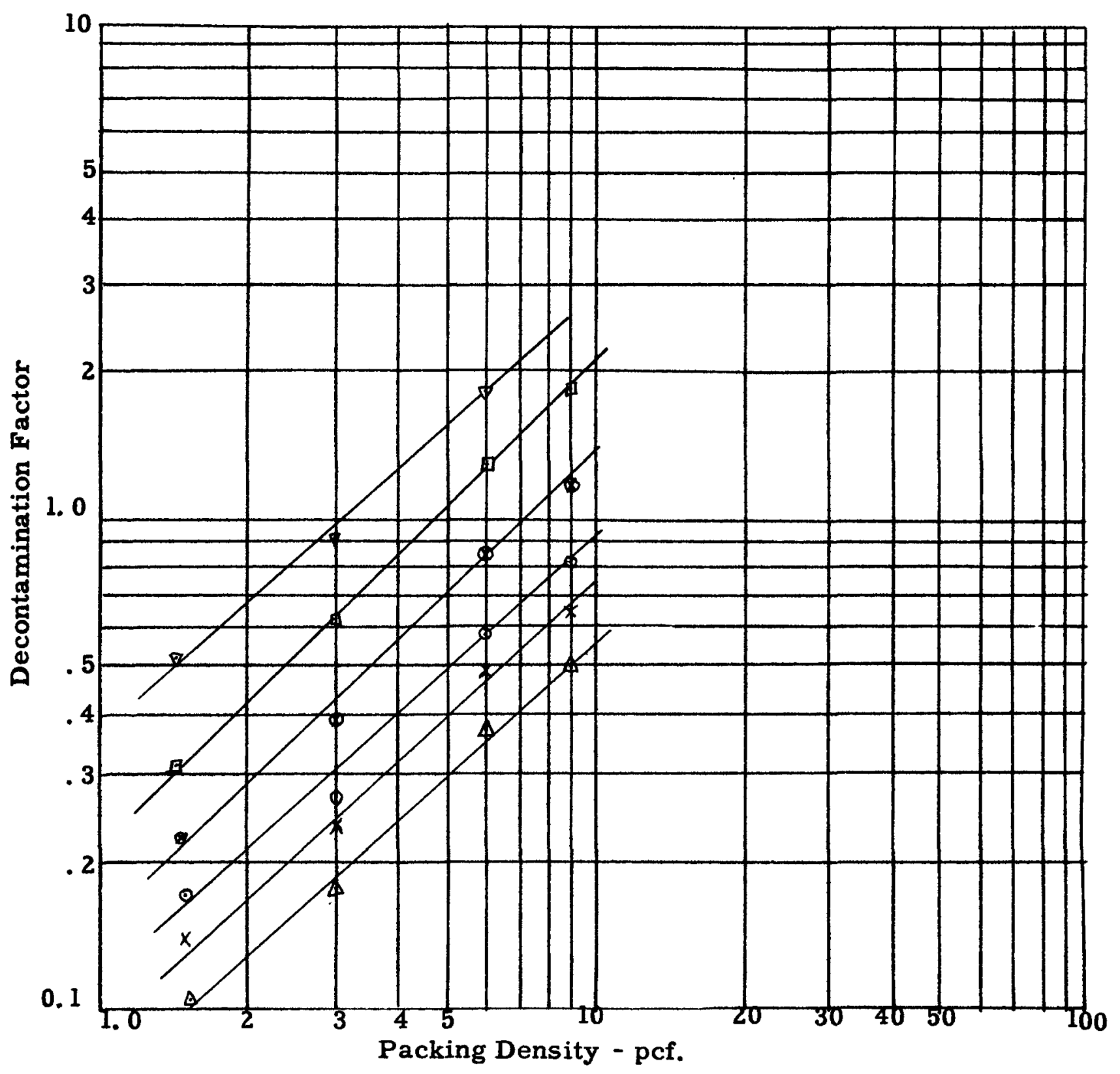



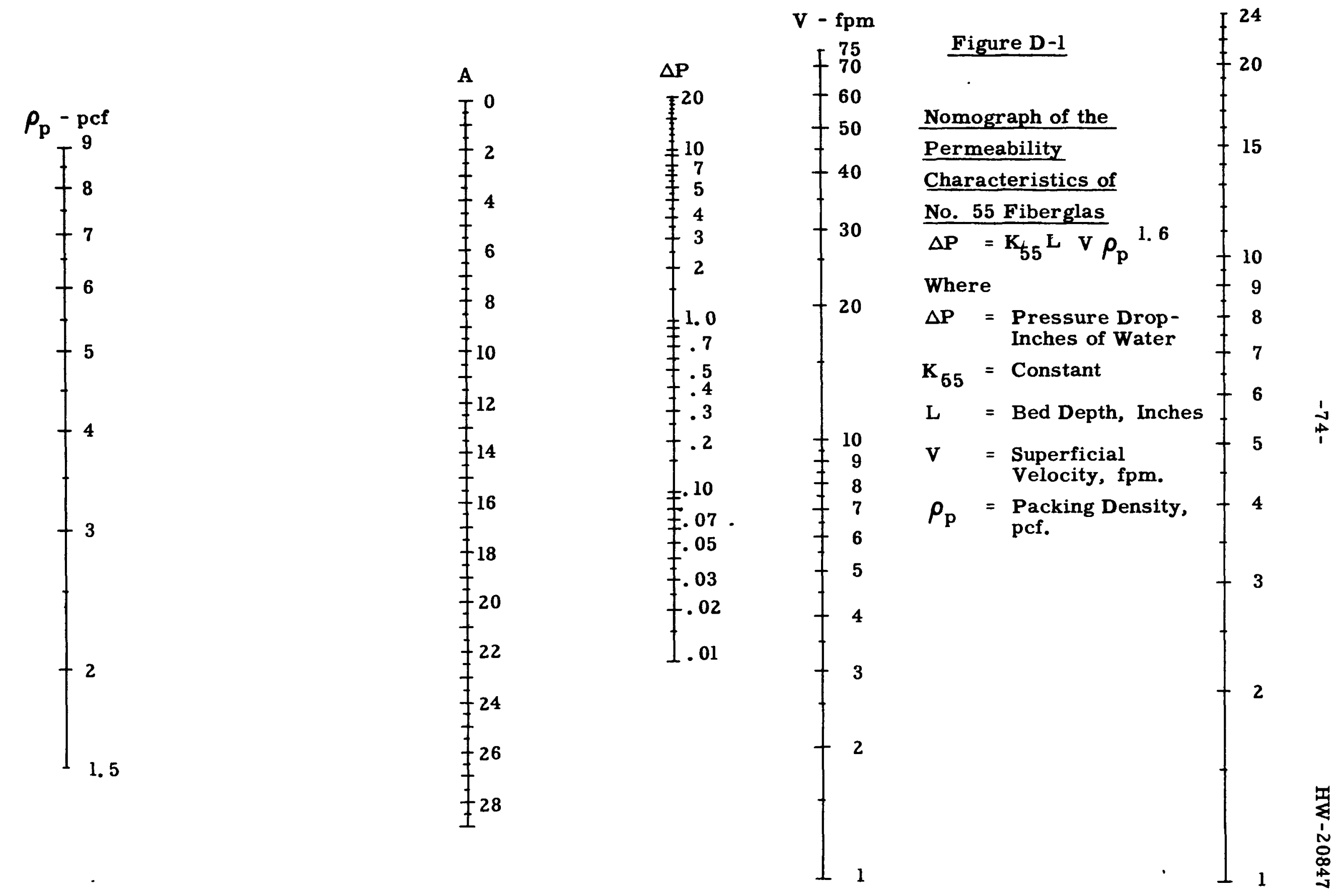


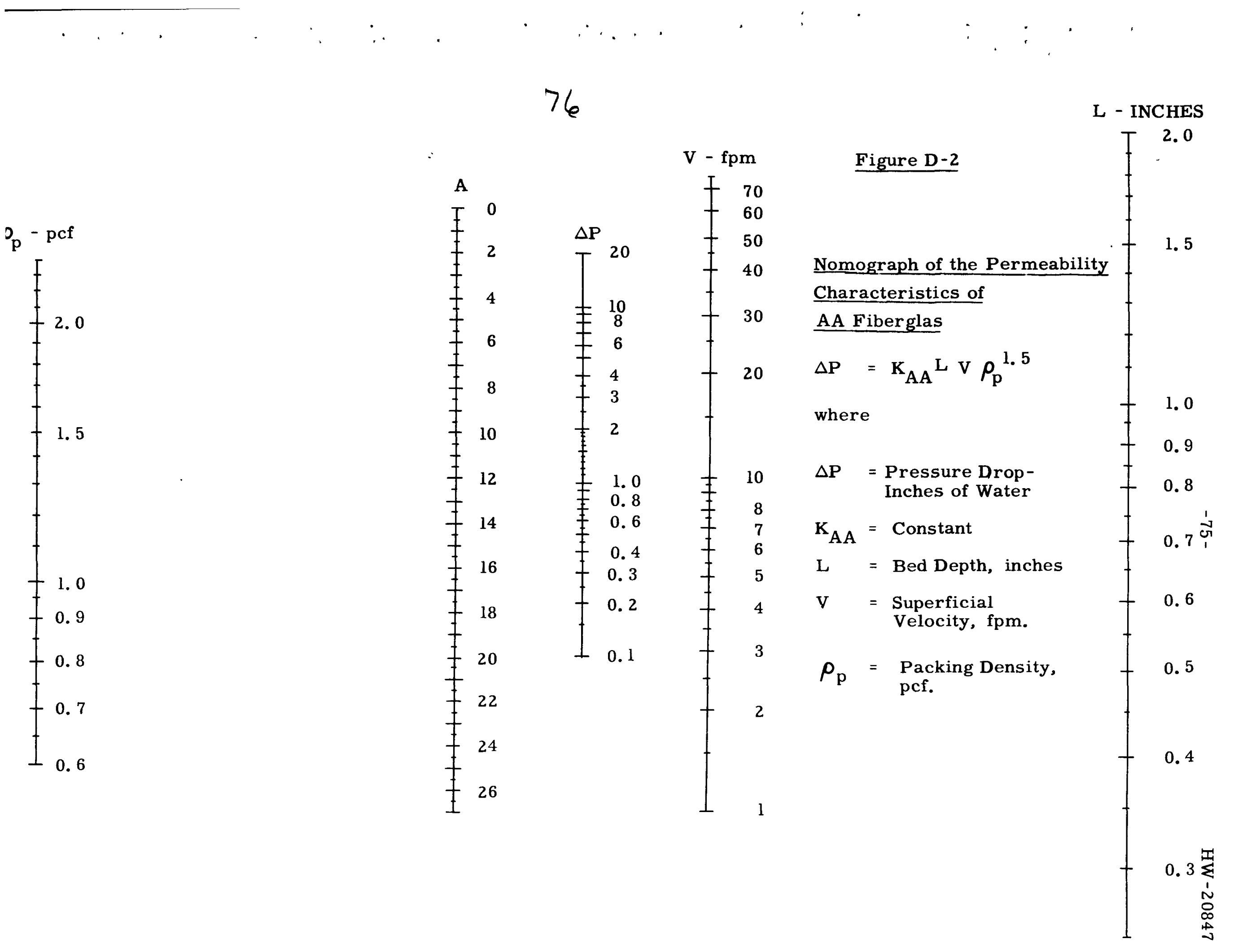




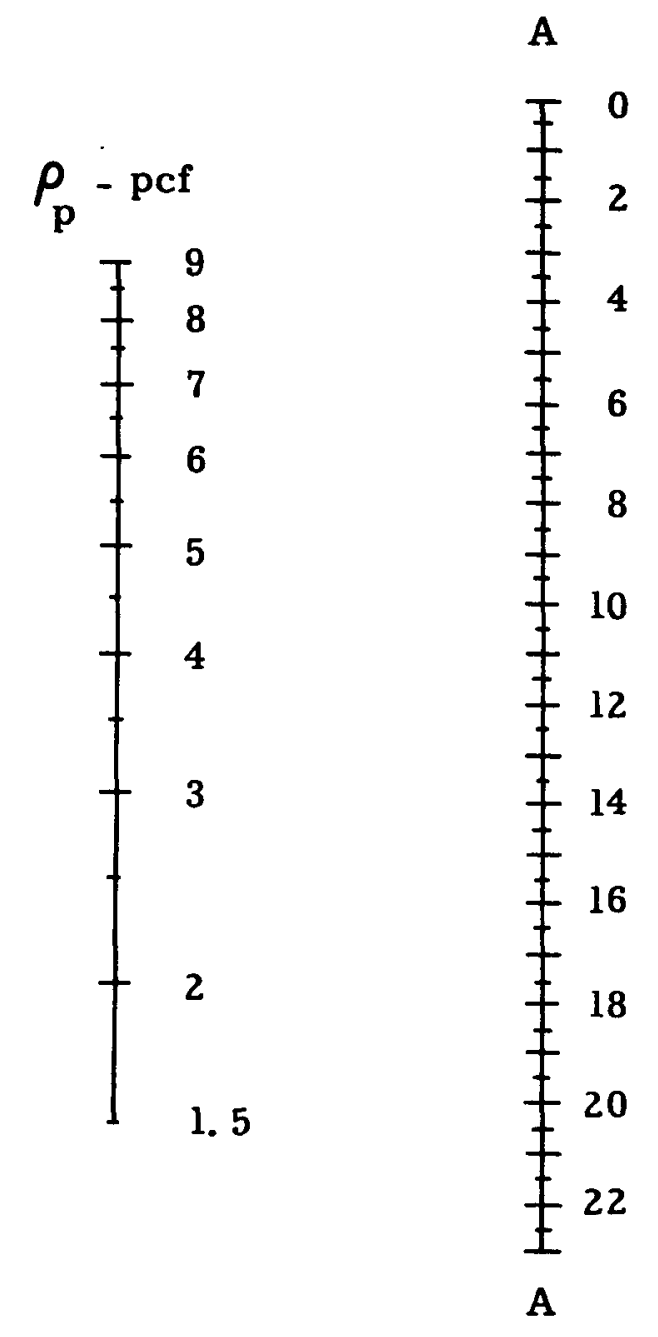

$$
\text { V - f pm }
$$
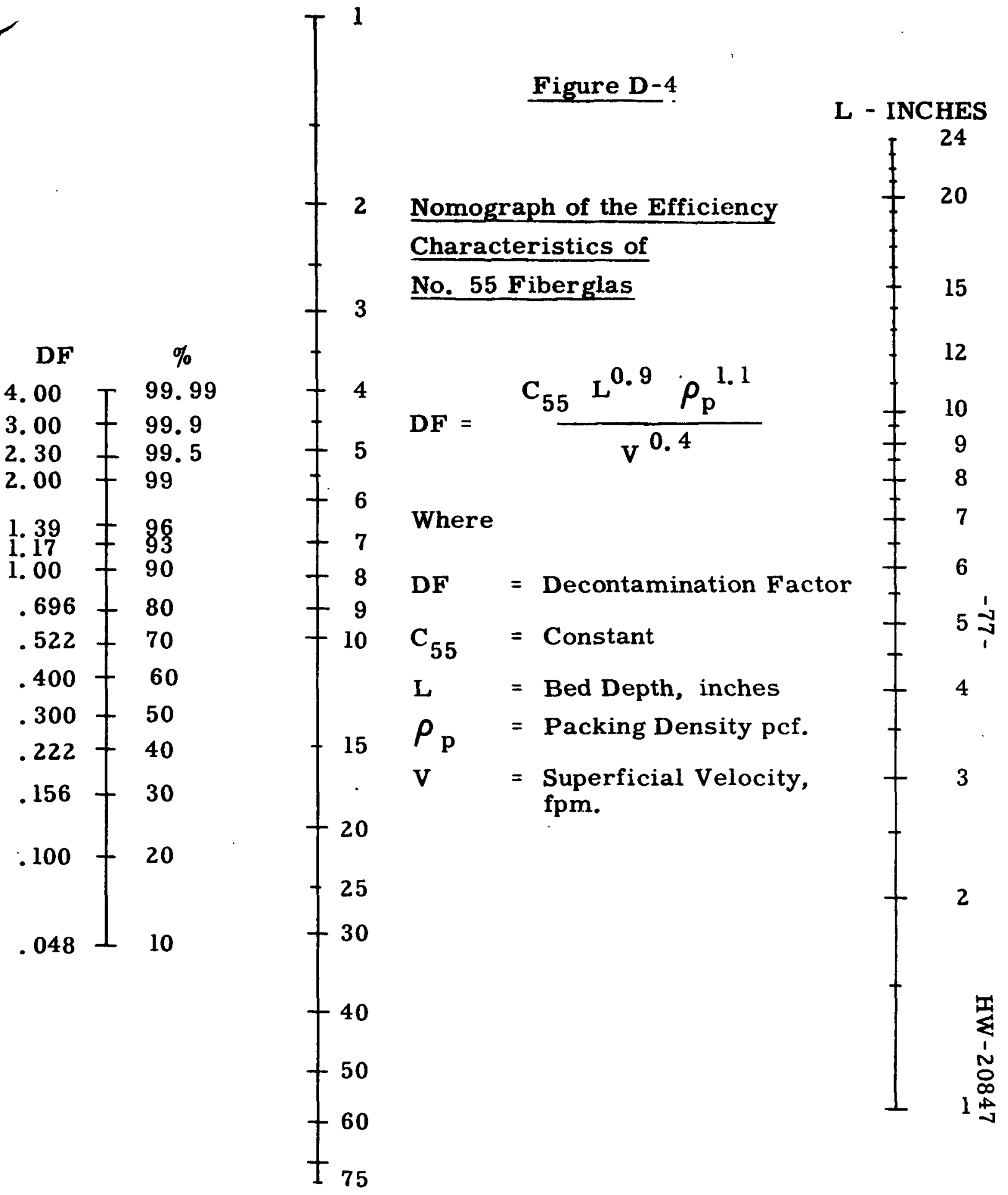
A

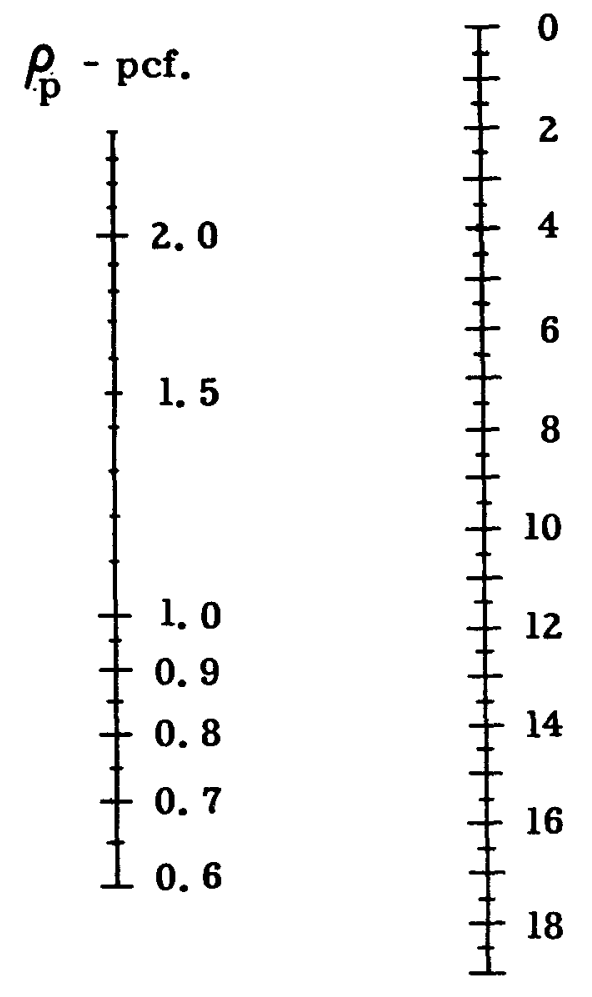

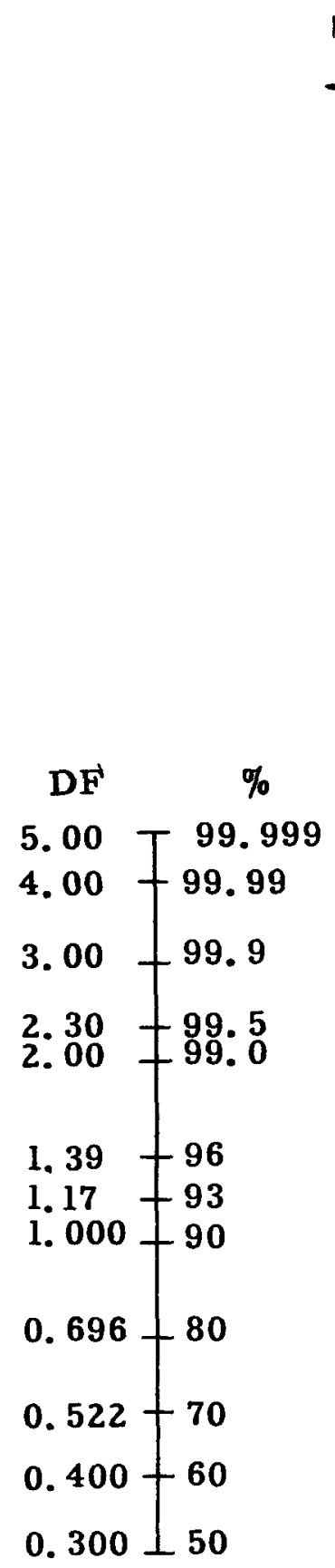

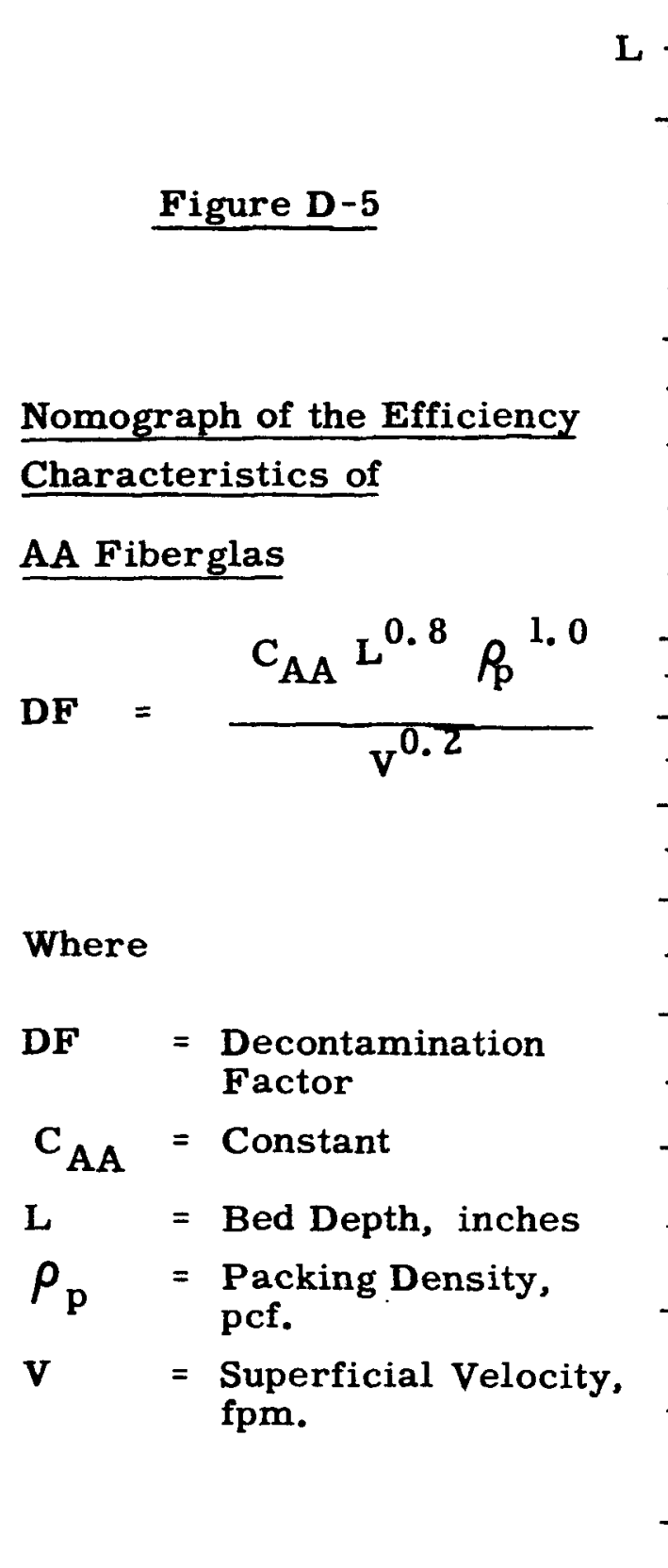

L - INCHES

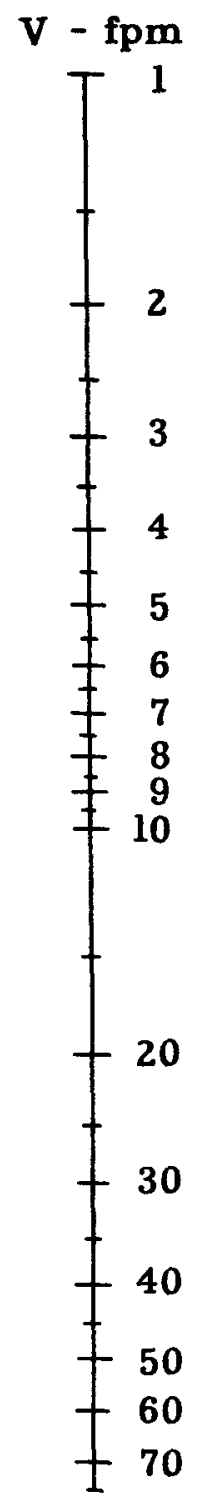

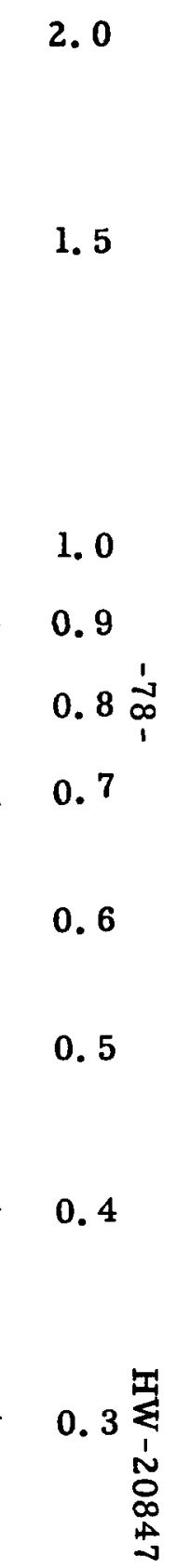



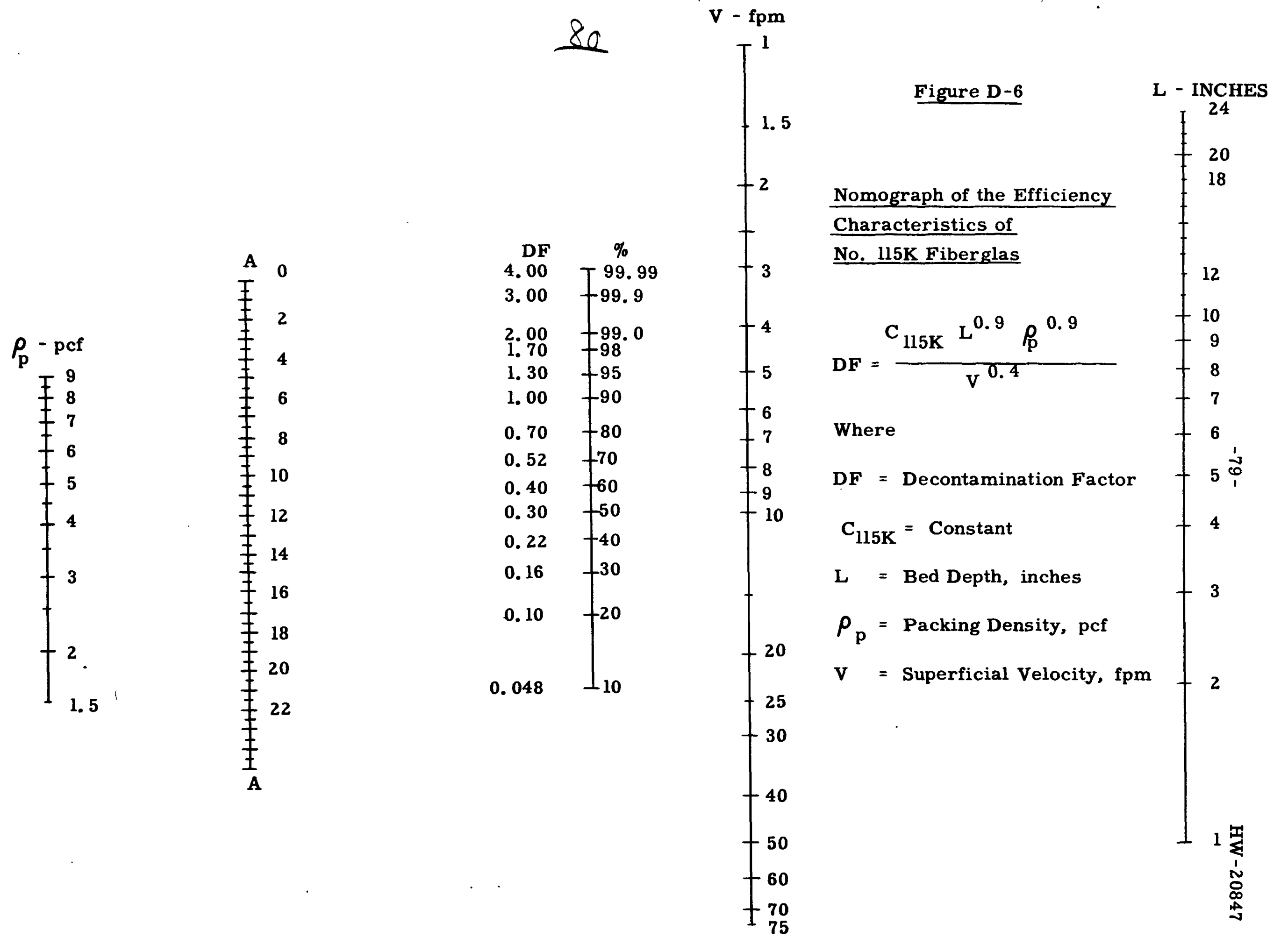
8)

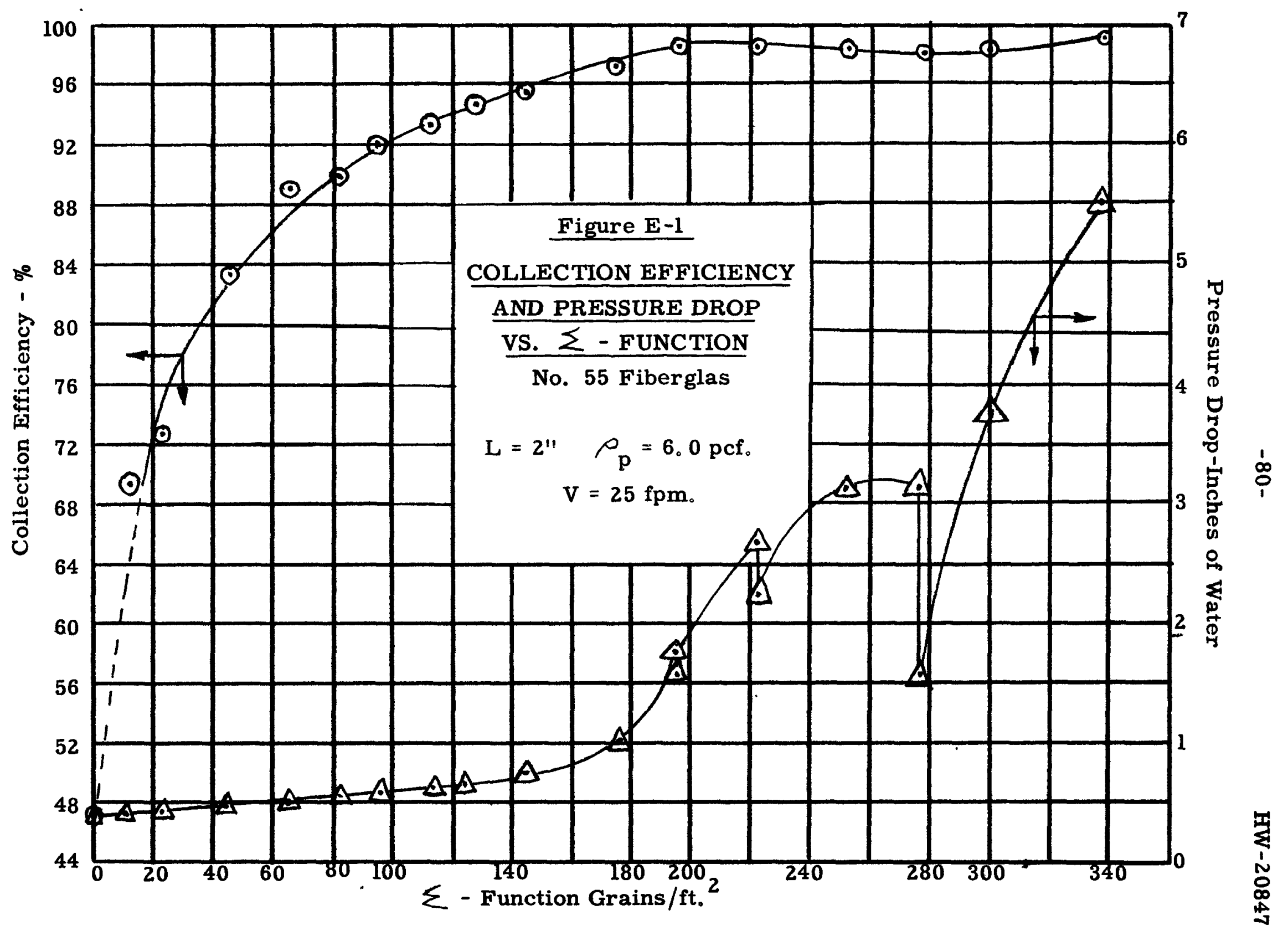




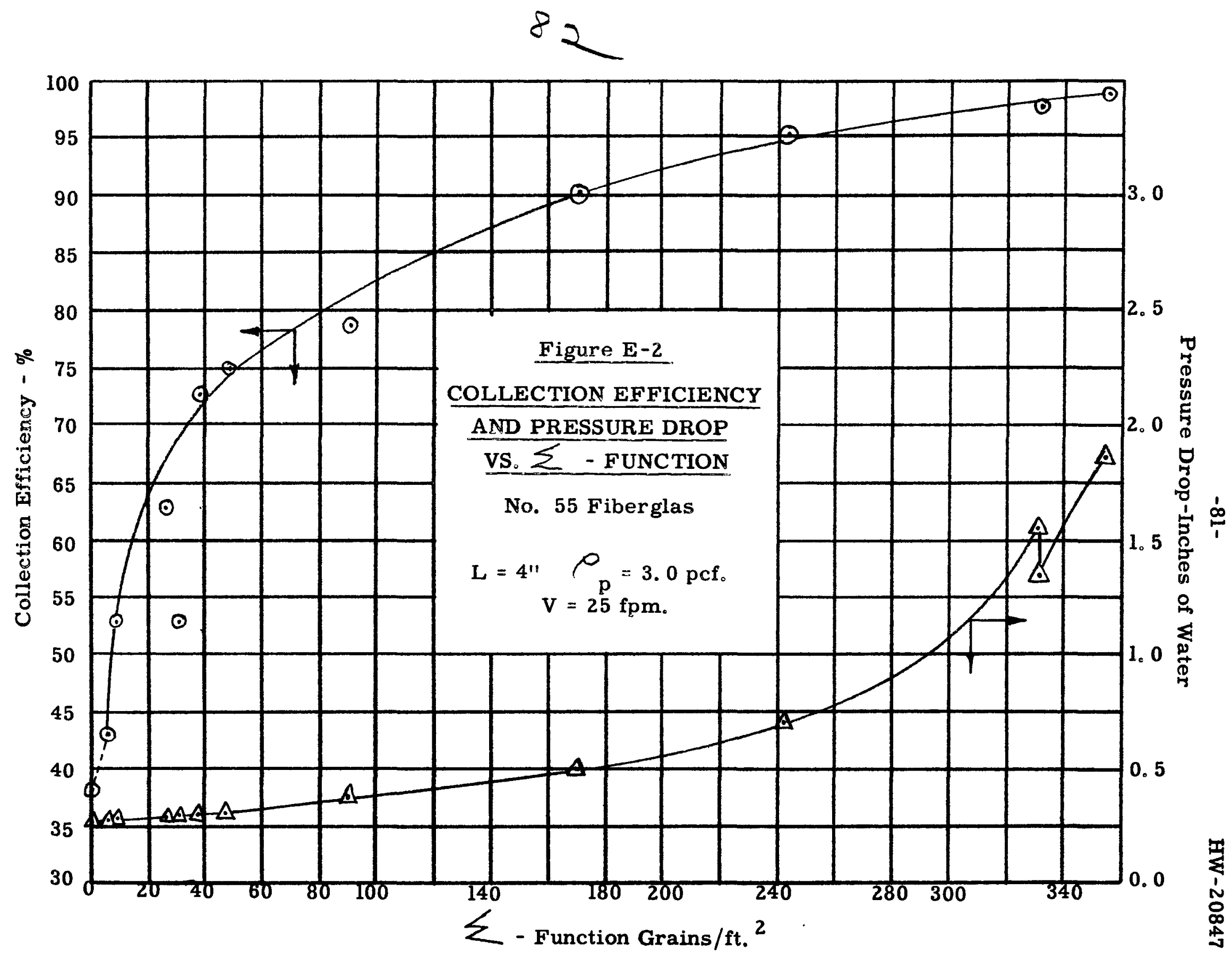




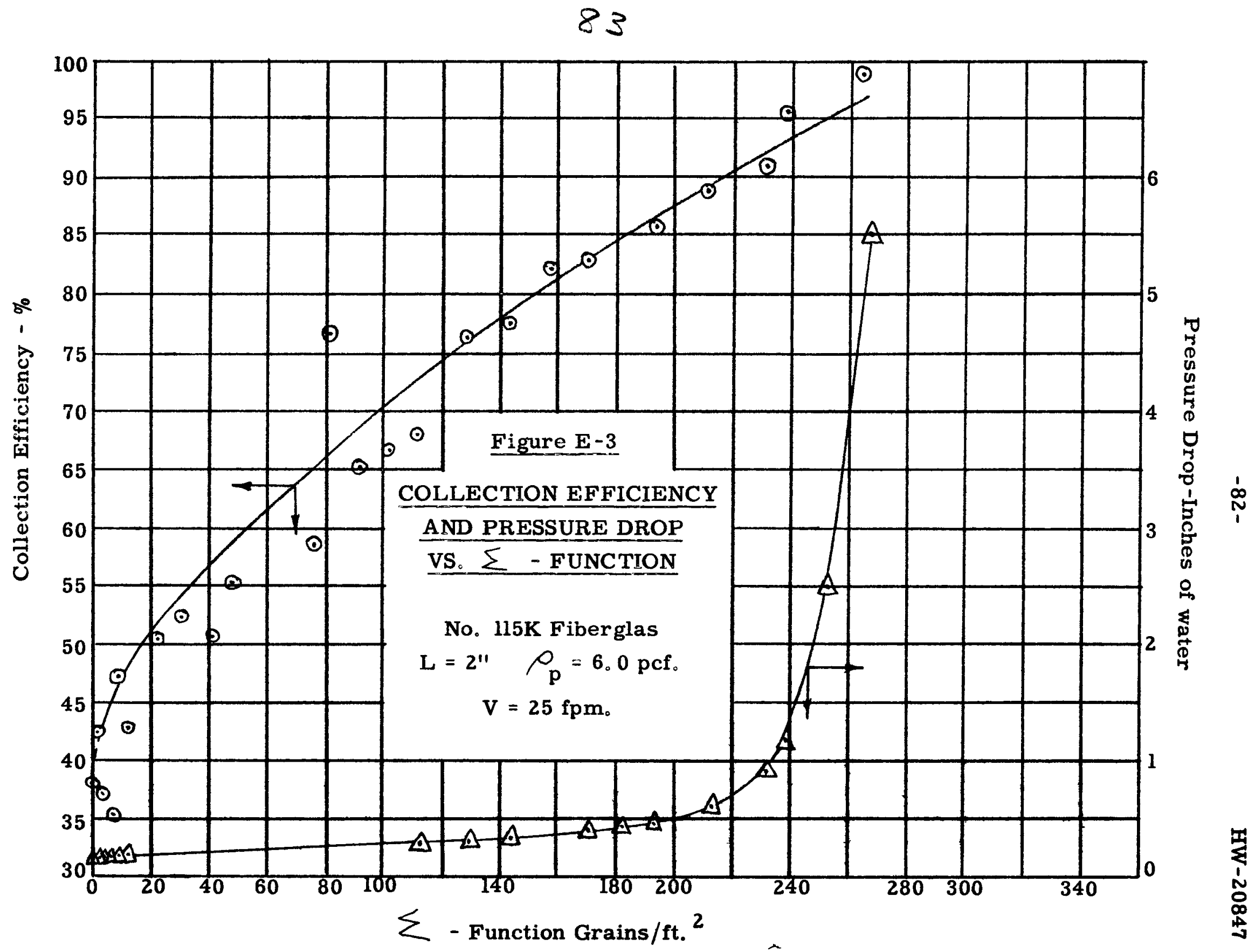


84

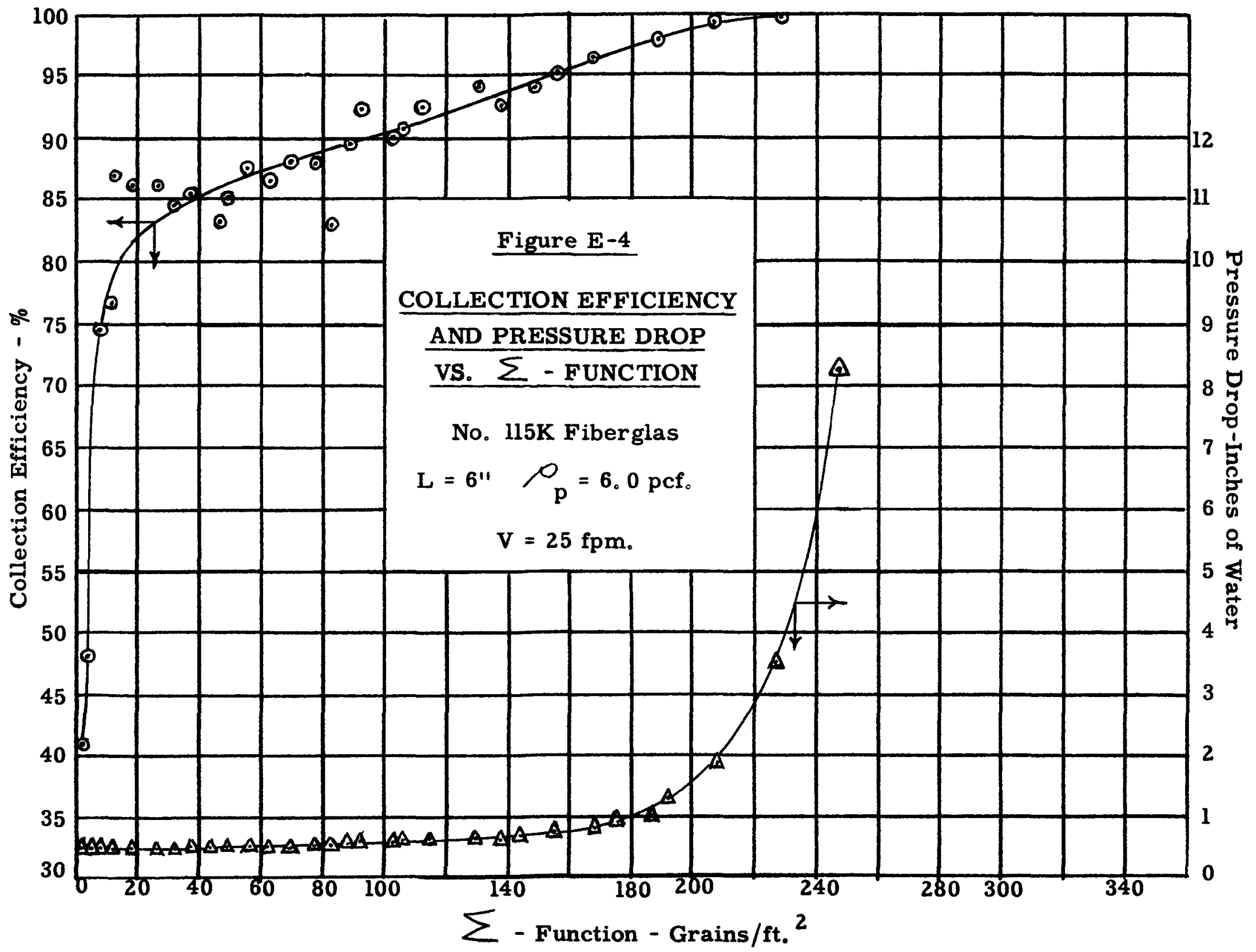




$$
-84-\quad 85
$$

Figure E-5

GRAPH OF PRESSURE DROP

VS. $\sum$ - FUNCTION FOR THE FIRST SAND FILTER TEST UNIT

0 - 2 inches of Type IV Sand

$\Delta-12$ inches of Type V Sand

$\square-3$ inches of Type VI Sand

$X$ - Entire filter unit

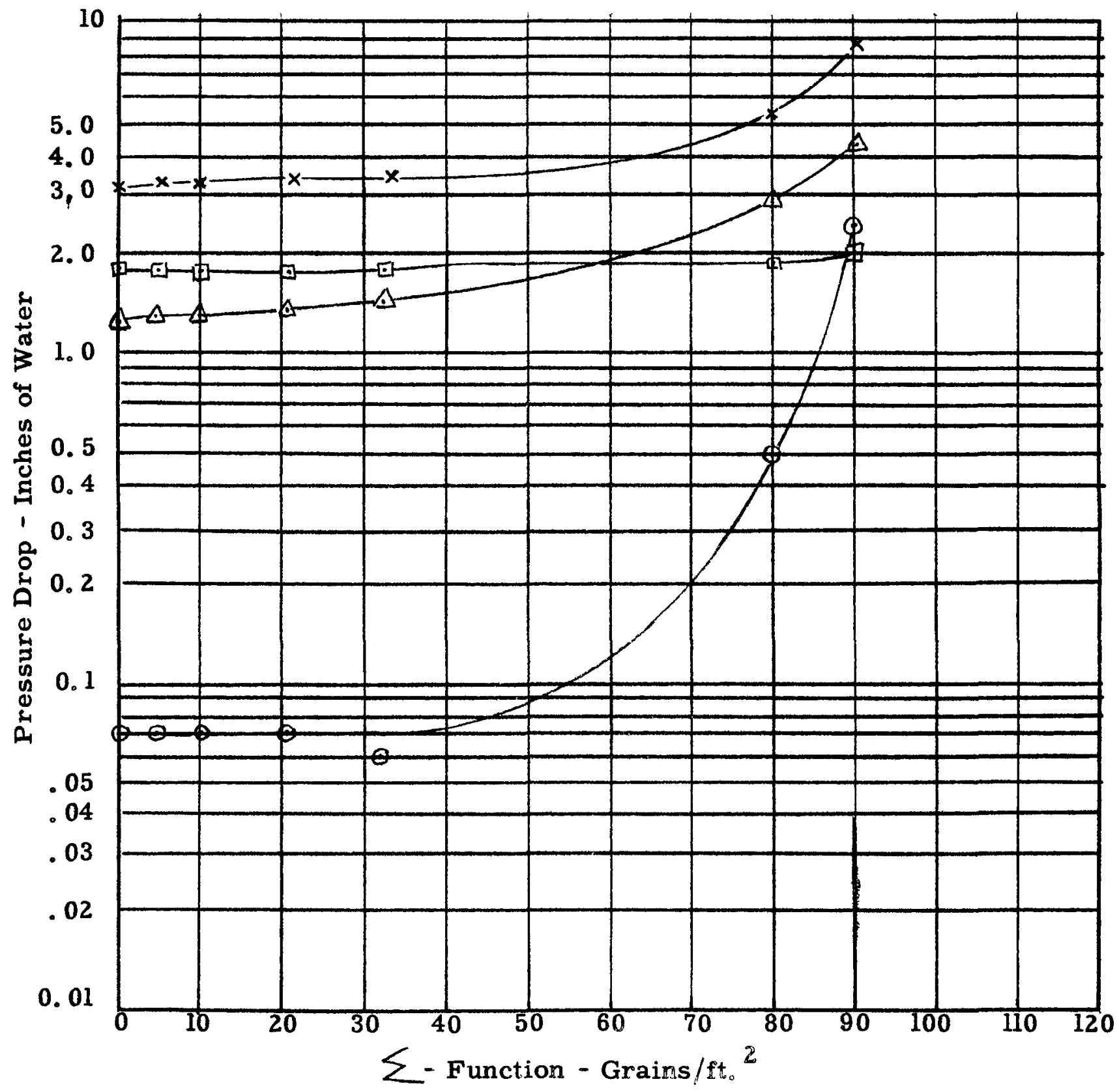




\section{6}

\section{7}

か

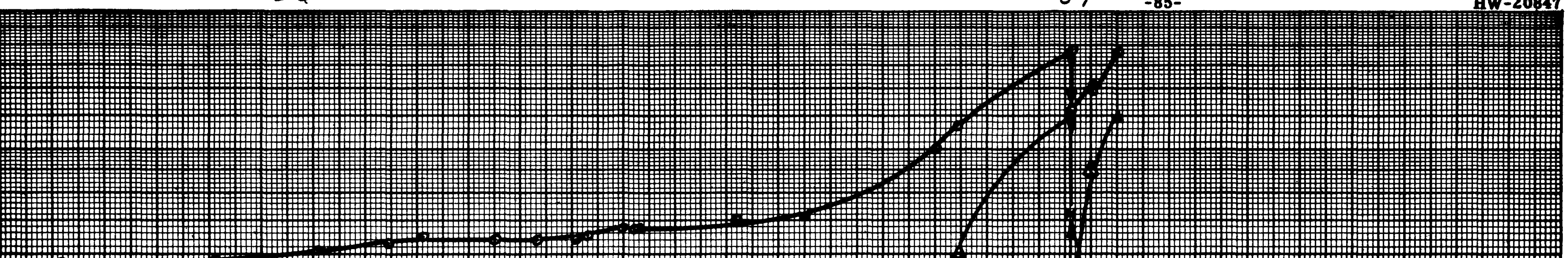

1.0 尚

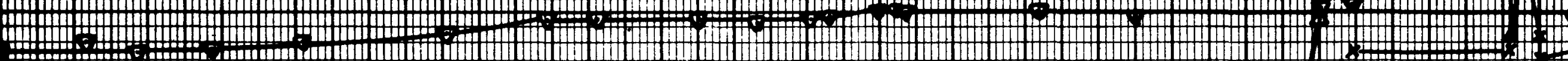

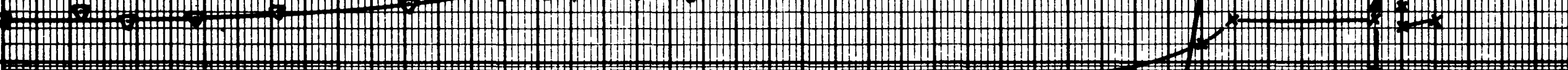

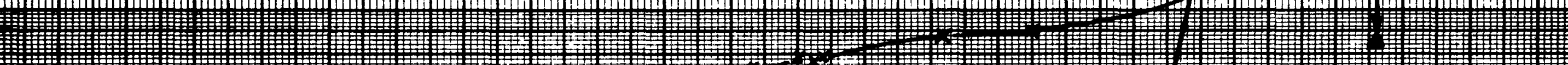

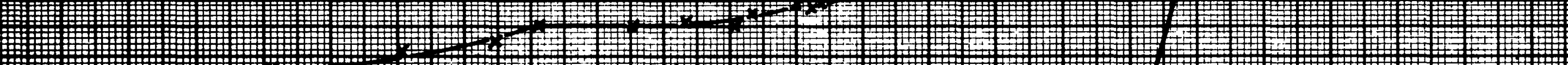

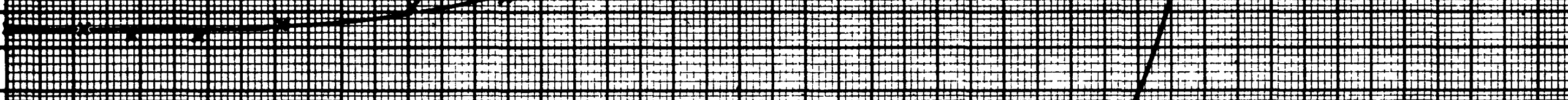

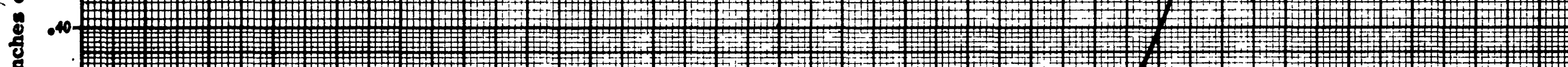

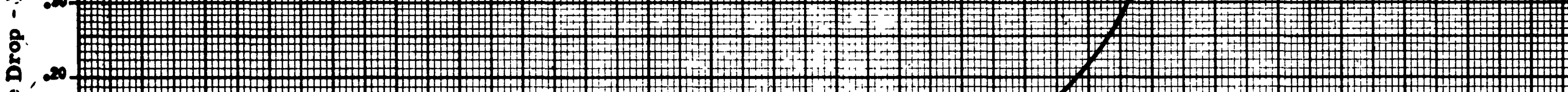
(m)

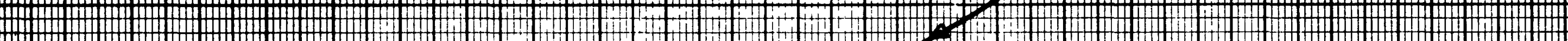
(1)

... \begin{tabular}{lll} 
& \\
\hline
\end{tabular}

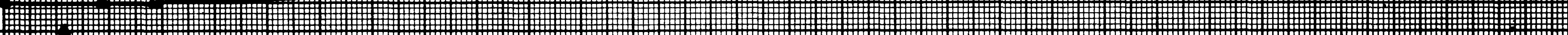

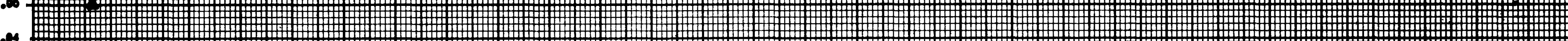

.03.

-

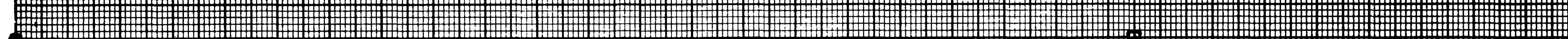

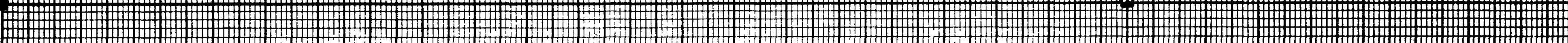

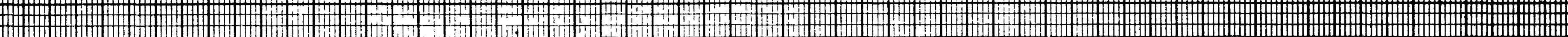

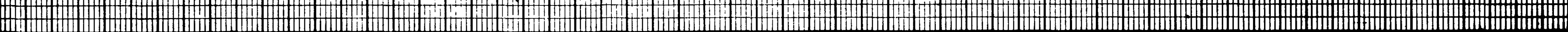

0.01 ${ }_{12}{ }_{10}{ }_{24}$ 

M
M M
M

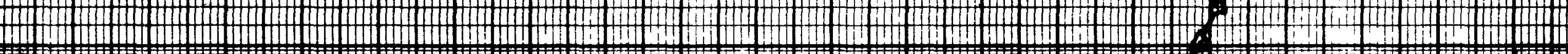
WV

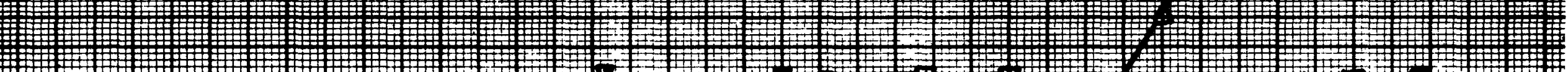
W W ${ }_{.0}^{.00}$ W W W ${ }^{x} 0$ W W W

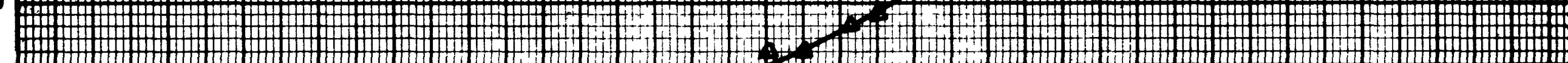
约 0.10 地,

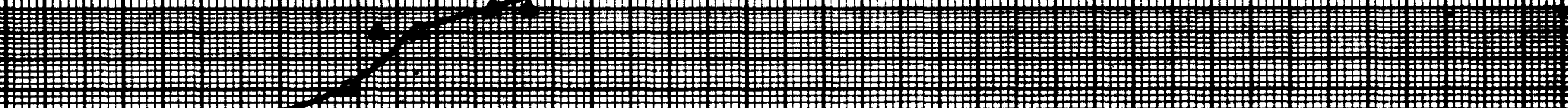

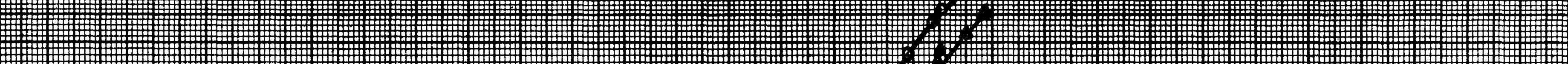
m 约

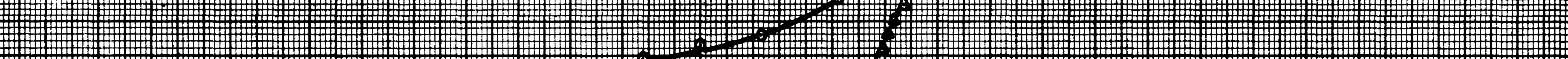
+m

GRAPH OF PRESSURE DROP

vS. $\Sigma$ - FUNCTION FOR THE THIRD SAND FILTER TEST UNIT

- II - Inches of Type II Sand

- Types II - III Interface $\odot-10$ Inches of Type III Sand ه - Types III - IV Interface (2) - 4 Inches of Type IV Sand $\Delta$ - Types IV - V Interface $\square-10$ Inches of Type $V$ Sand $X$ - Types V - VI Interface $\nabla-2$ Inches of Type VI Sand 0 - Entire Filter Unit

Superficial Velocity - 5 fpm.

Interfacial layers extended one inch above and one inch below the line of demarcation.

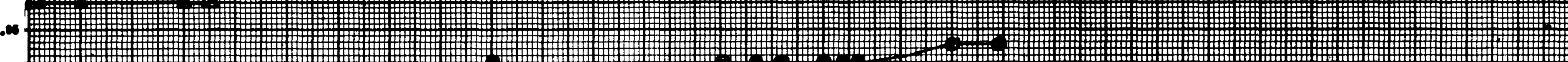

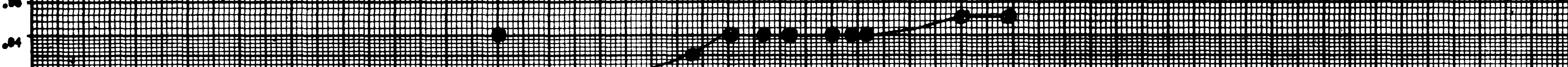

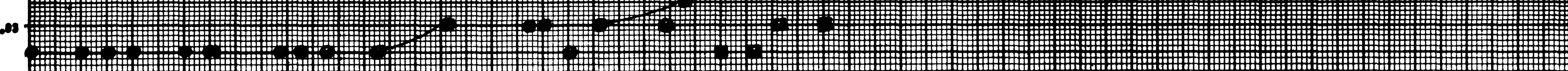

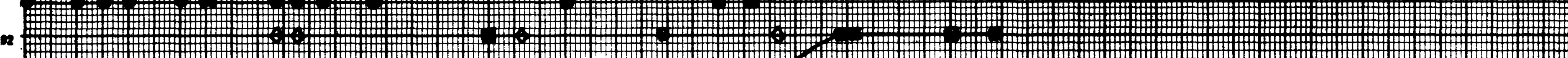

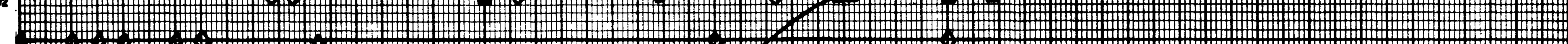

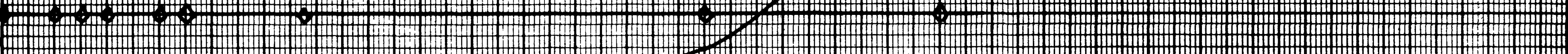

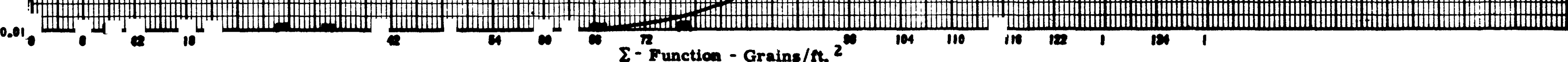




\section{0}

\section{9)}

$-87-$

GRAPH OF THE RADIOACTIVITY DISTRIBUTION

IN A PLANT SAND FILTER

O - Tube No. 1
$\mathrm{X}$ - Tube No. 2

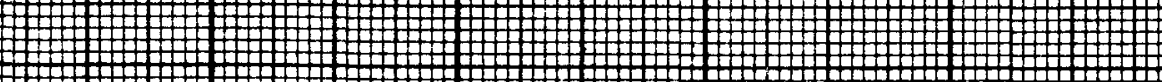

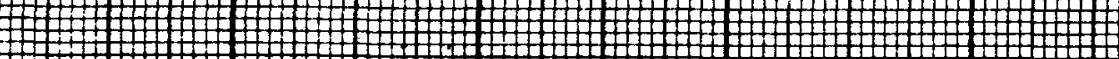

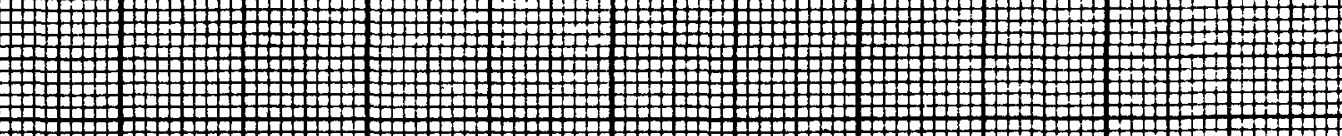

J
J

J

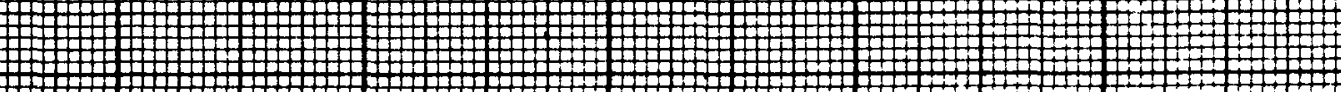

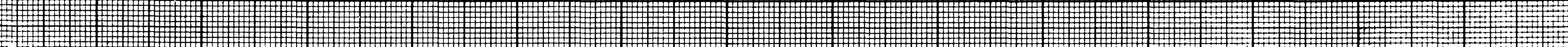

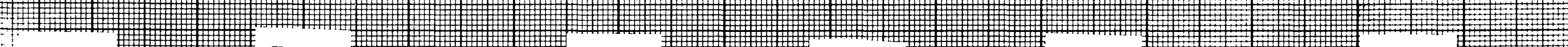
TypeI

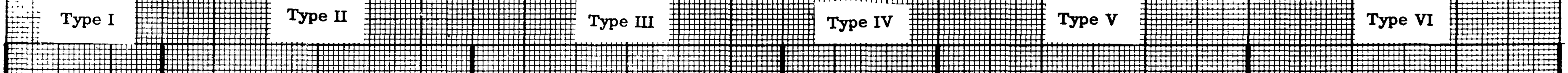
l $+1$

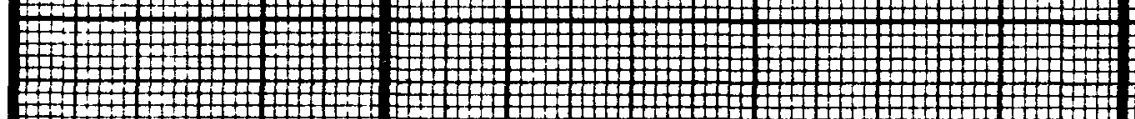

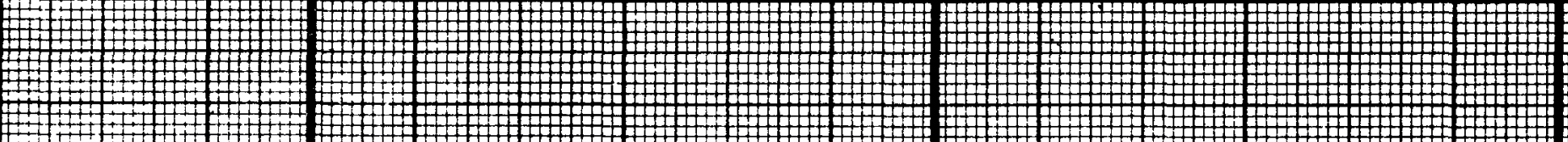
(J)

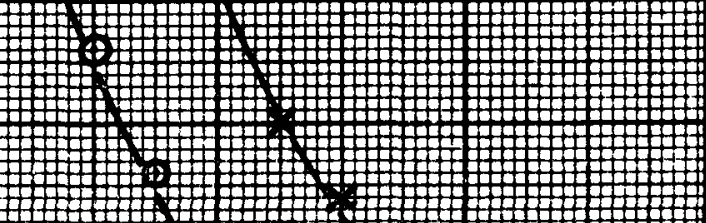


$-88-$

92

$\mathrm{HW}-20847$

Figure E-9

GRAPH OF PRESSURE DROP

VS. $\sum$ - FUNCTION

O - 5 inches of No. 55 Fiberglas at 9.0 pcf. Protecting

$\Delta-0.25$ inch of AA Fiberglas at 1.2 pcf.

Superficial Velocity - $50 \mathrm{fpm}$.

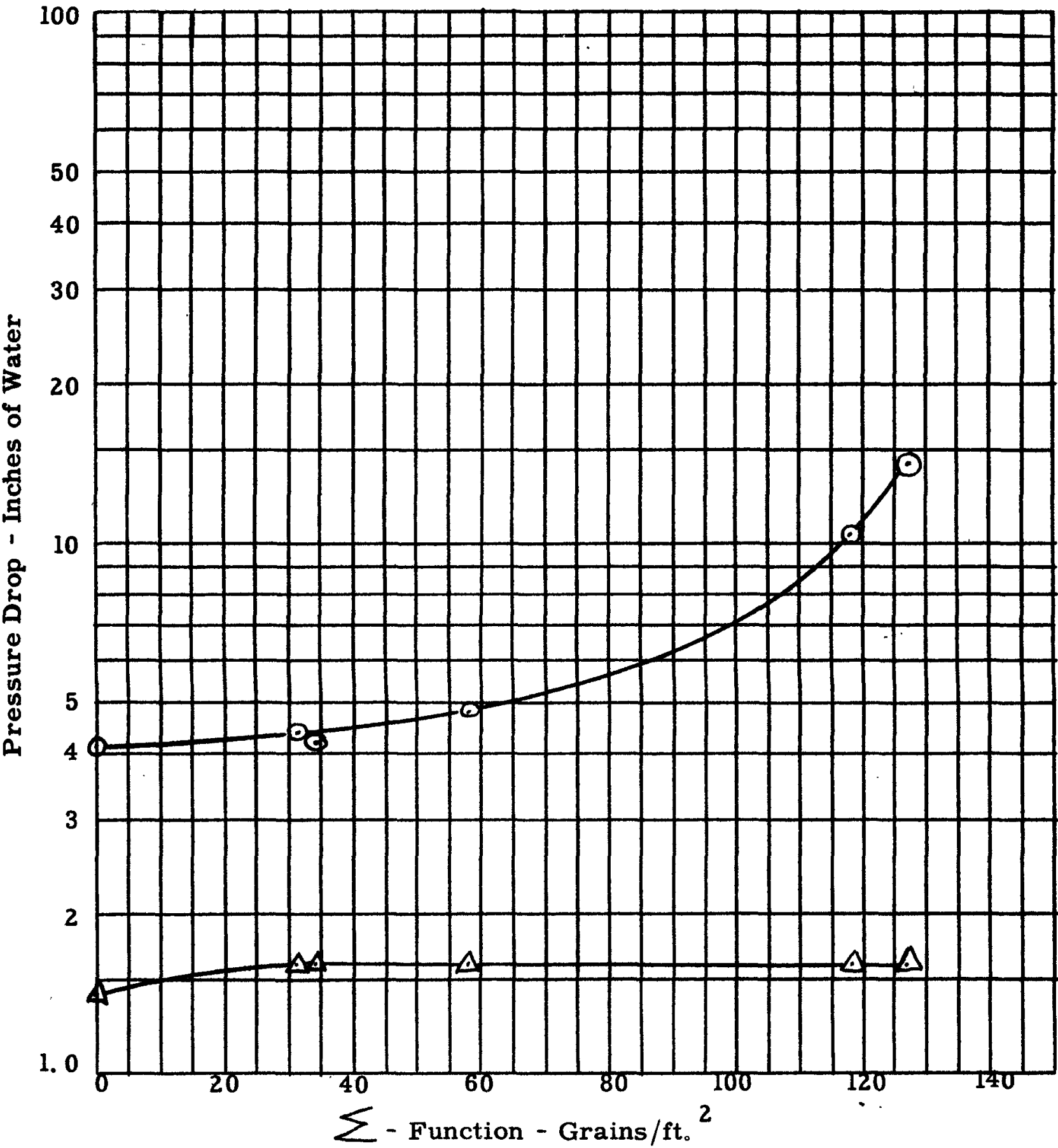


Figure E-10

GRAPH OF PRESSURE DROP

$$
\text { VS. } \sum \text { - FUNCTION }
$$

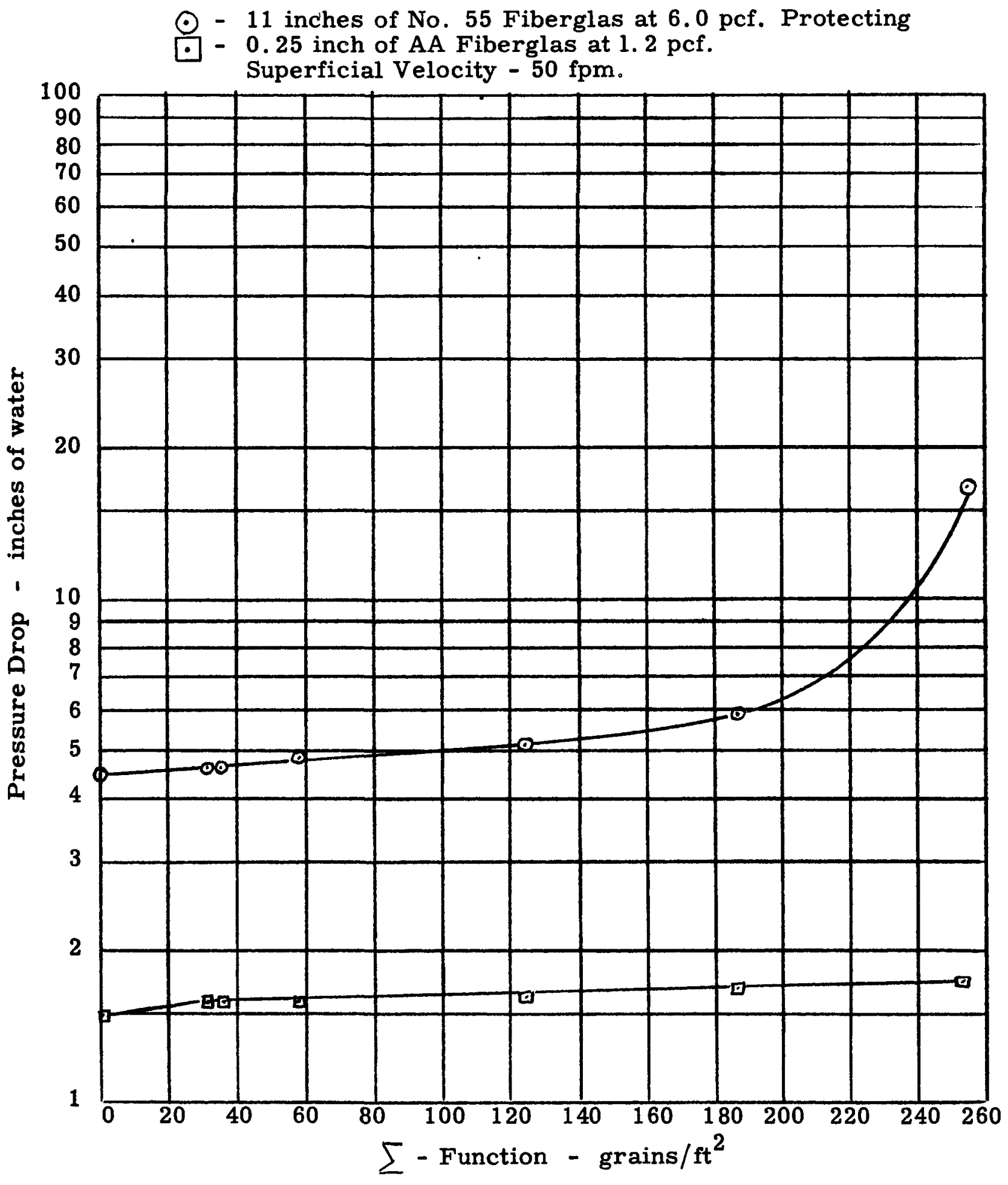




$$
-90-94
$$

Figure E-11

GRAPH OF PRESSURE DROP

$$
\text { VS。 } \Sigma \text { - FUNCTION }
$$

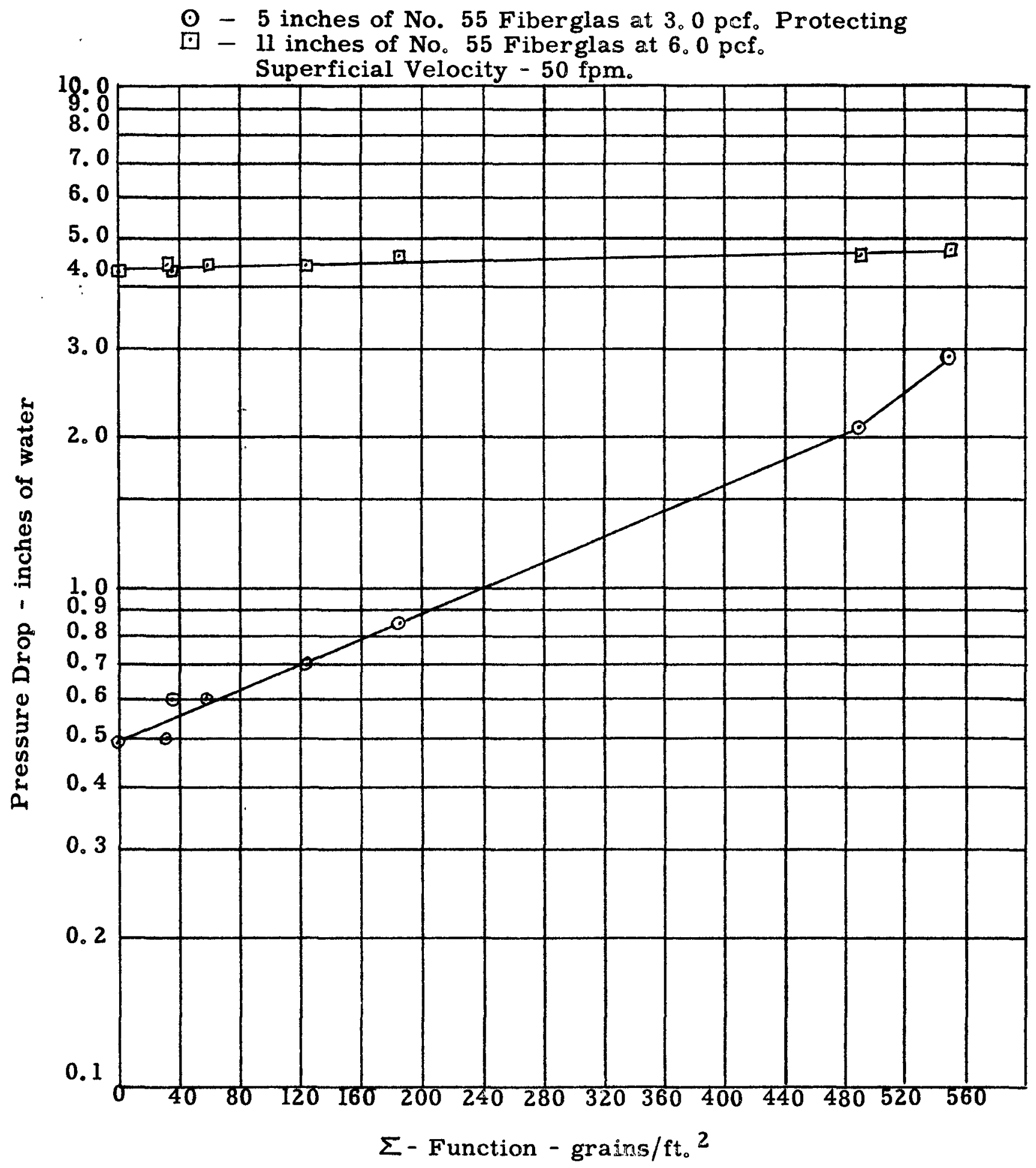




$$
-91-\quad 95
$$

Figure E-12

GRAPH OF PRESSURE DROP

VS. $\Sigma$ - FUNCTION

口-12 inches of No。 450 Fiberglas at 5.7 pcf. Protecting

$\odot-5$ inches of No. 55 Fiberglas at 3.0 pcf. Superficial Velocity - 50 fpm.

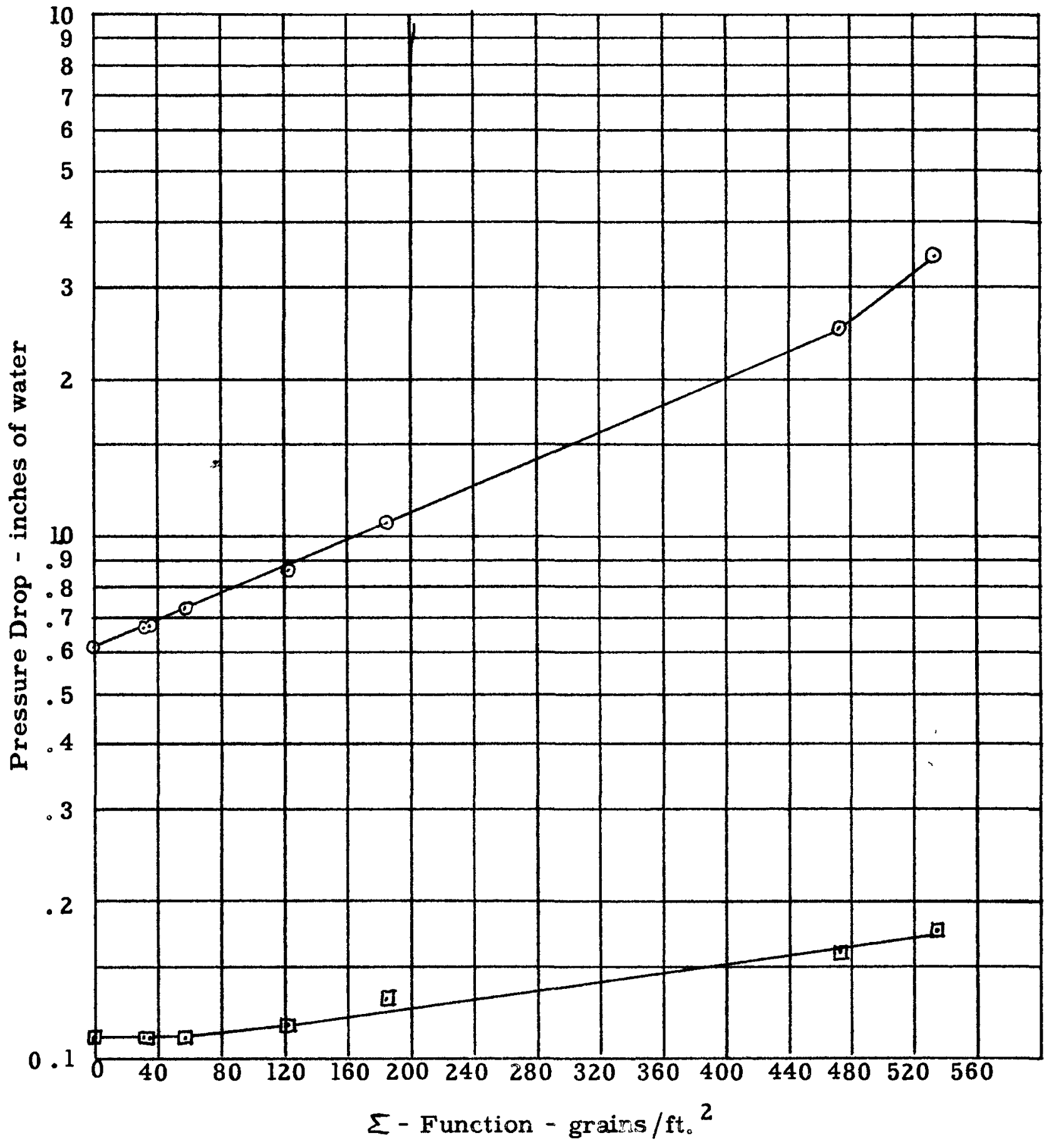




$$
-92-96
$$

Figure E-13

GRAPH OF PRESSURE DROP

VS. $\Sigma-$ FUNCTION

$\odot-12$ inches of No, 600 Fiberglas at 6.8 pcf. Protecting

๑ 12 inches of No. 450 Fiberglas at 5.7 pcf. Superficial Velocity - 50 fpm.

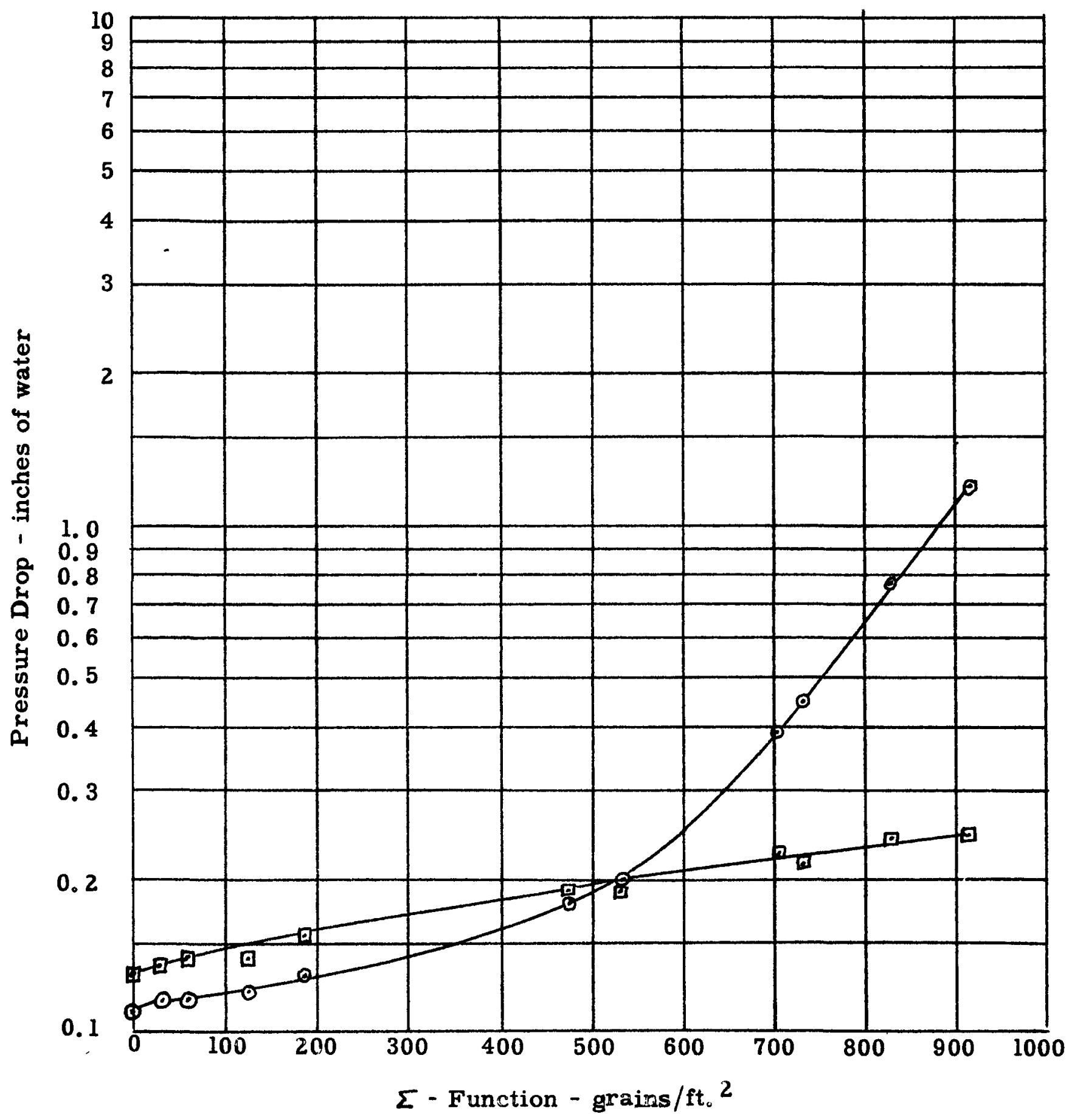




$$
-93-
$$

Figure E-14

GRAPH OF PRESSURE DROP

$$
\text { VS。 } \Sigma \text { - FUNCTION }
$$

๑ -12 inches of No. 800 Fiberglas at 6,6 pcf. Protecting

$\odot-12$ inches of No. 600 Fiberglas at 6.8 pcf. Superficial Velocity - 50 fpm.

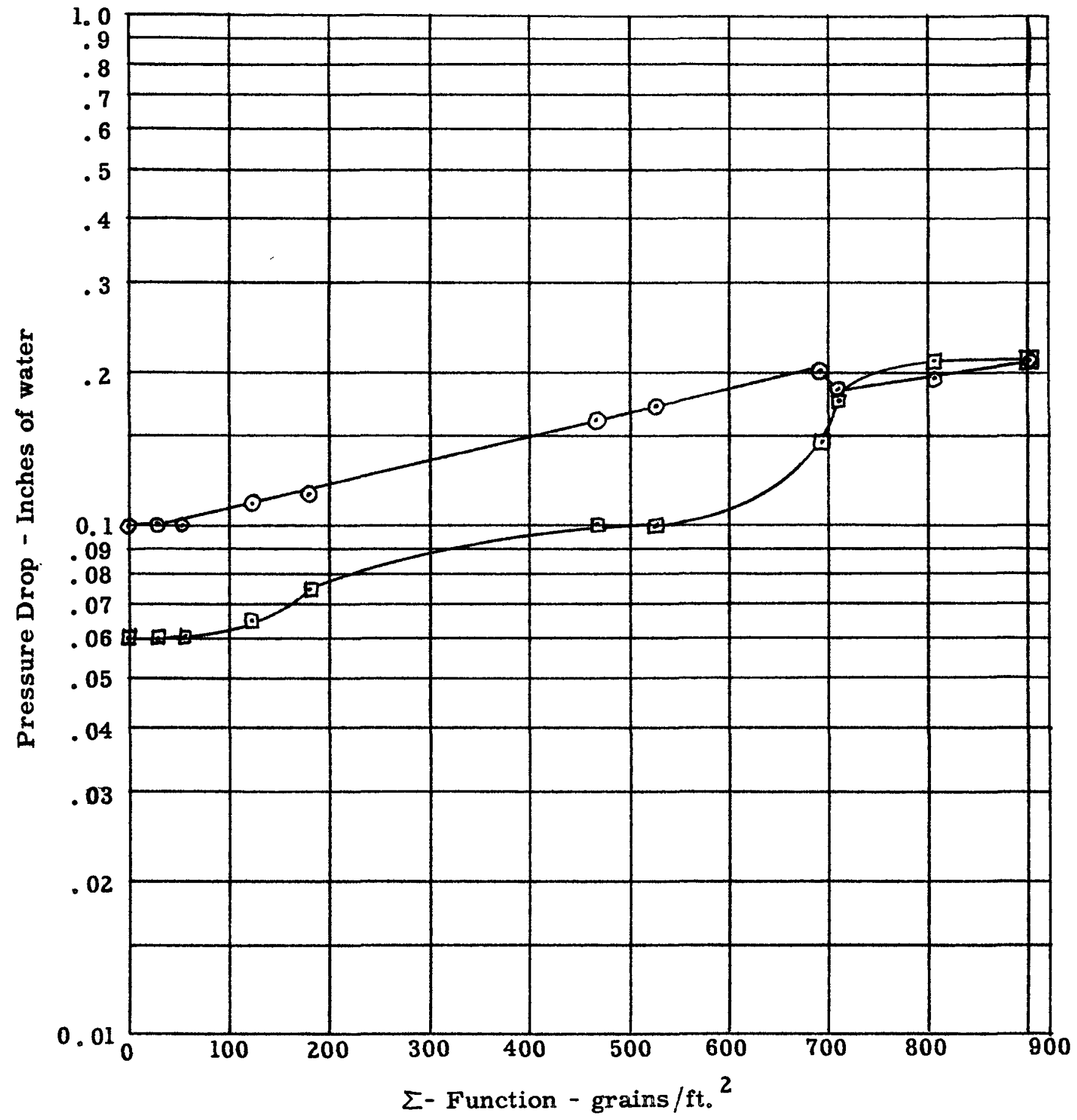


Figure E-15

GRAPH OF PRESSURE DROP

\section{VS. $\Sigma$ - FUNCTION}

口- 8 inches of No. 55 Fiberglas at 6.0 pcf. Protecting

$0-0.25$ inch of AA Fiberglas at $] .2$ pcf. Superficial Velocity - $25 \mathrm{fpm}$.

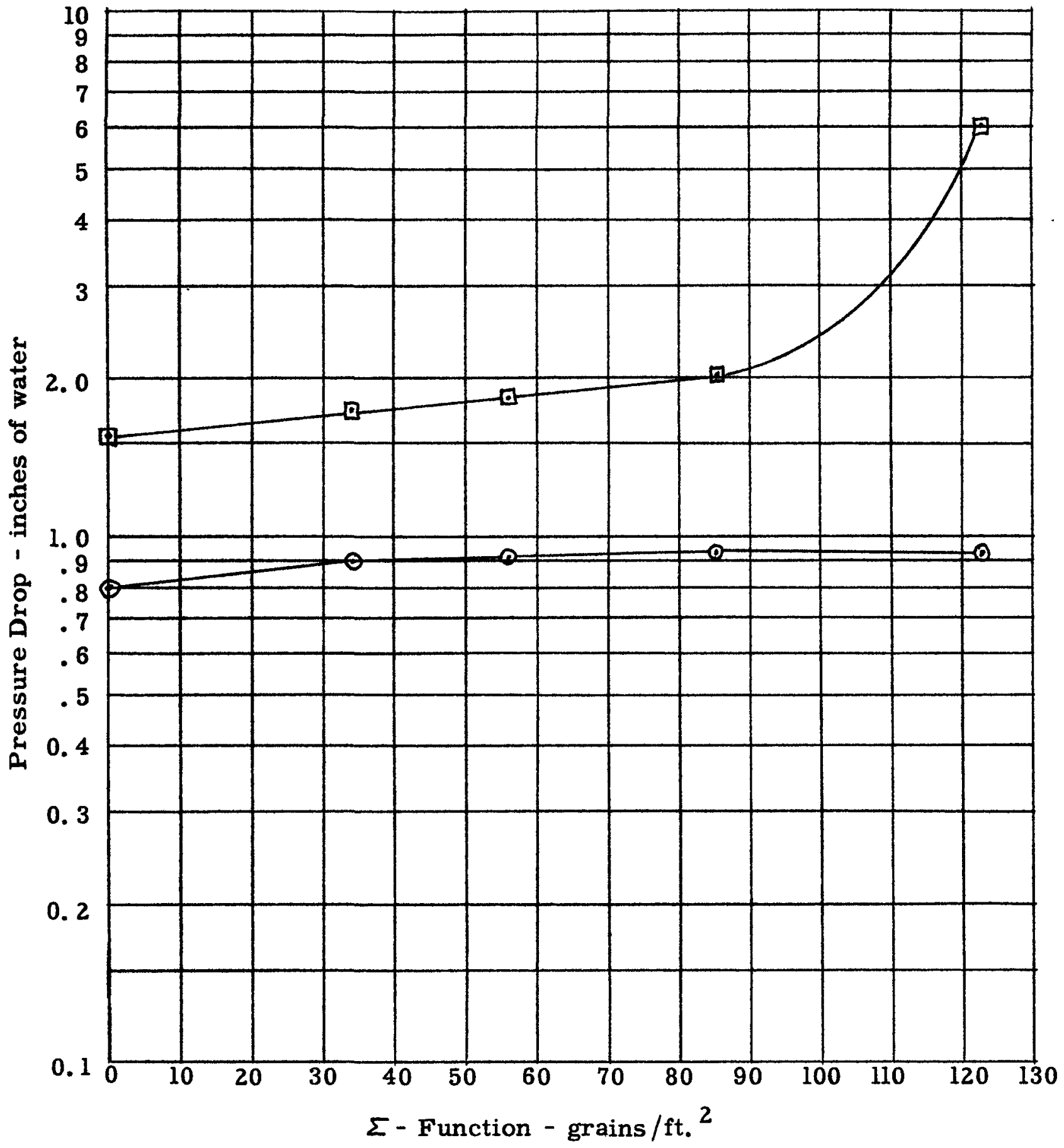




$$
-95-
$$

Figure E-16

GRAPH OF PRESSURE DROP VS. $\Sigma-$ FUNCTION

$\square-5$ inches of No. 55 Fiberglas at 3.0 pcf。Protecting

$\odot-8$ inches of No. 55 Fiberglas at 6.0 pcf。 Superficial Velocity - $25 \mathrm{fpm}$ 。

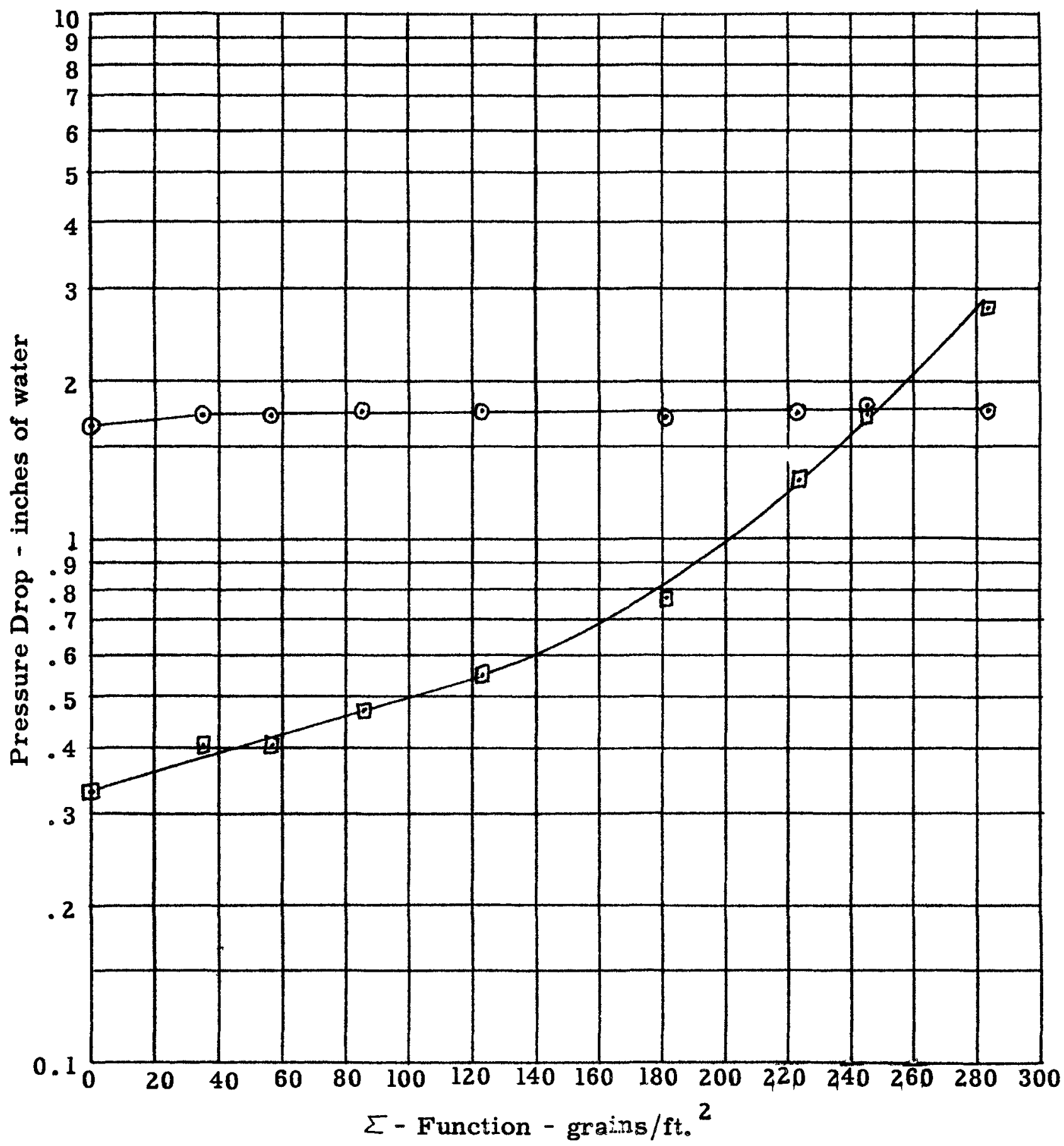




$$
-96-
$$$$
100
$$

Figure - $\mathbf{E}-17$

GRAPH OF PRESSURE DROP

\section{VS. $\Sigma$ - FUNCTION}

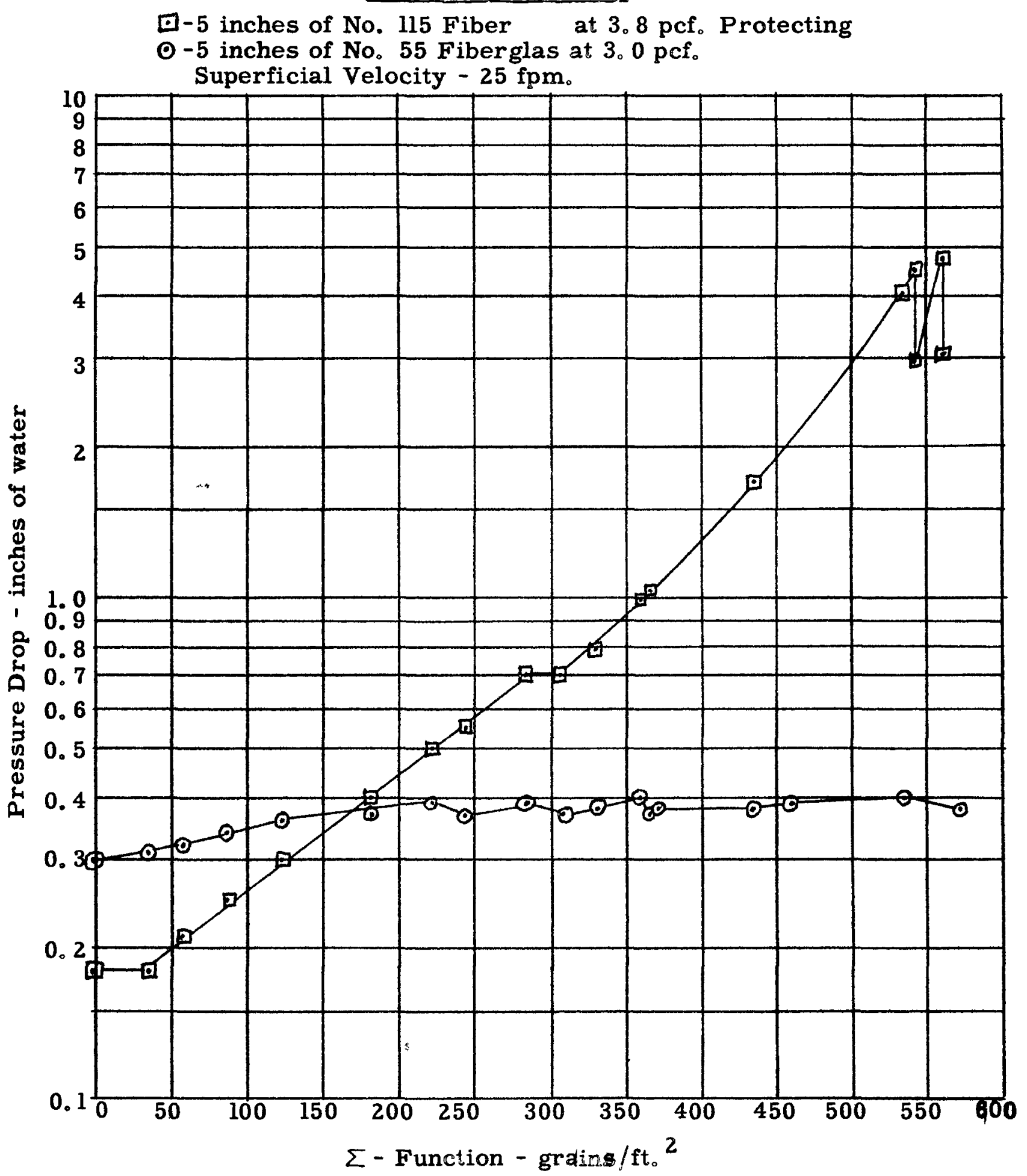


Figure E-18

GRAPH OF PRESSURE DROP

\section{VS. $\Sigma-$ FUNCTION}

$\odot-12$ inches of No. 450 Fiberglas at 10 pcf. Protecting

$\square-5$ inches of No。 115 Fiber at 3.8 pcf. Superficial Velocity - 25 fpm.

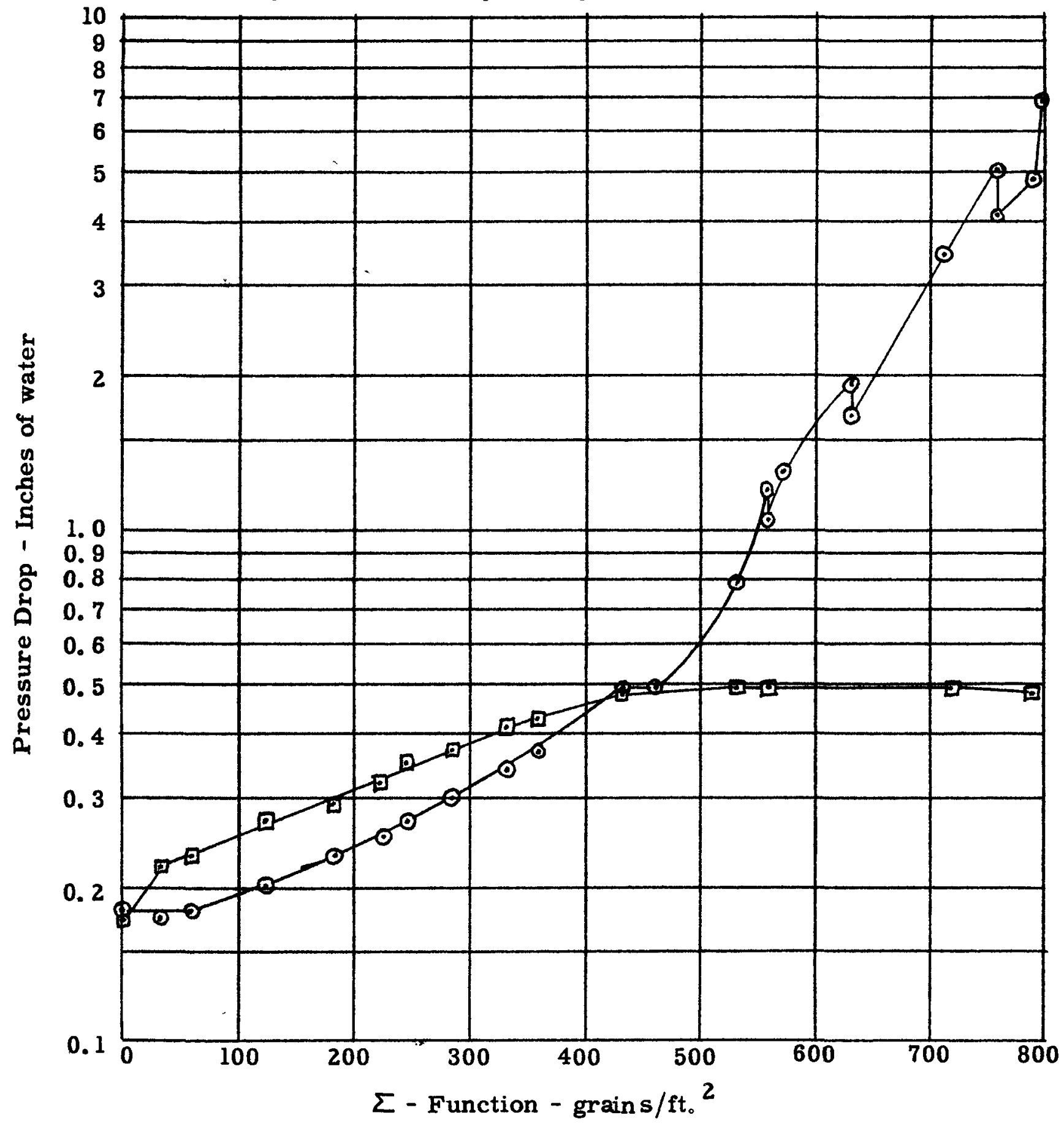




$$
-99-\quad .104
$$

Figure E-20

GRAPH OF PRESSURE DROP

VS. $\Sigma$ - FUNCTION

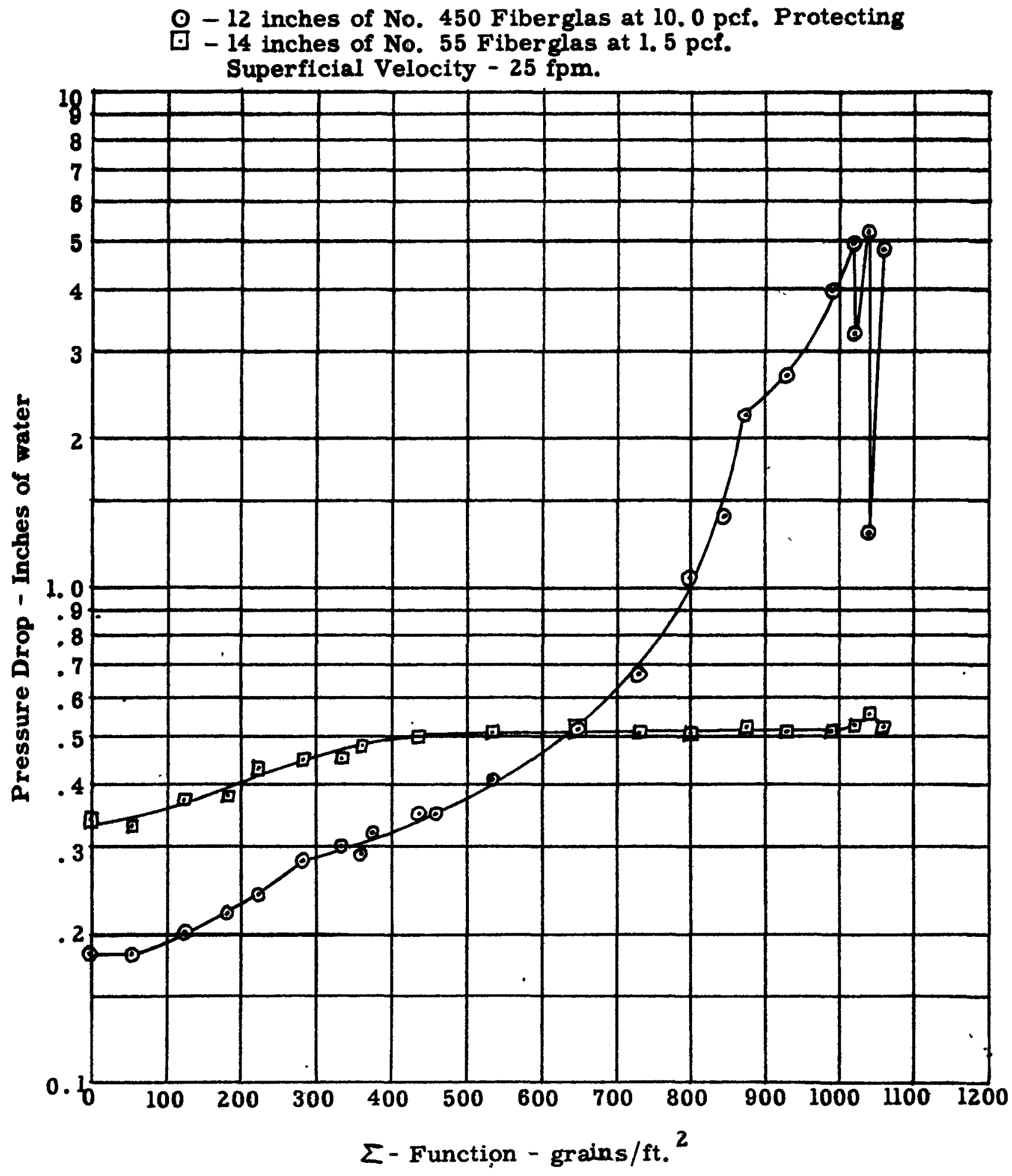


Figure E-21

GRAPH OF PRESSURE DROP

VS. $\Sigma$ - FUNCTION

$\odot-24$ inches No. 450 Fiberglas at 5. 0 pcf. Protecting Đ-14 inches No. 55 Fiberglas at 1.5 pcf.

Superficial Velocity $25 \mathrm{fpm}$.

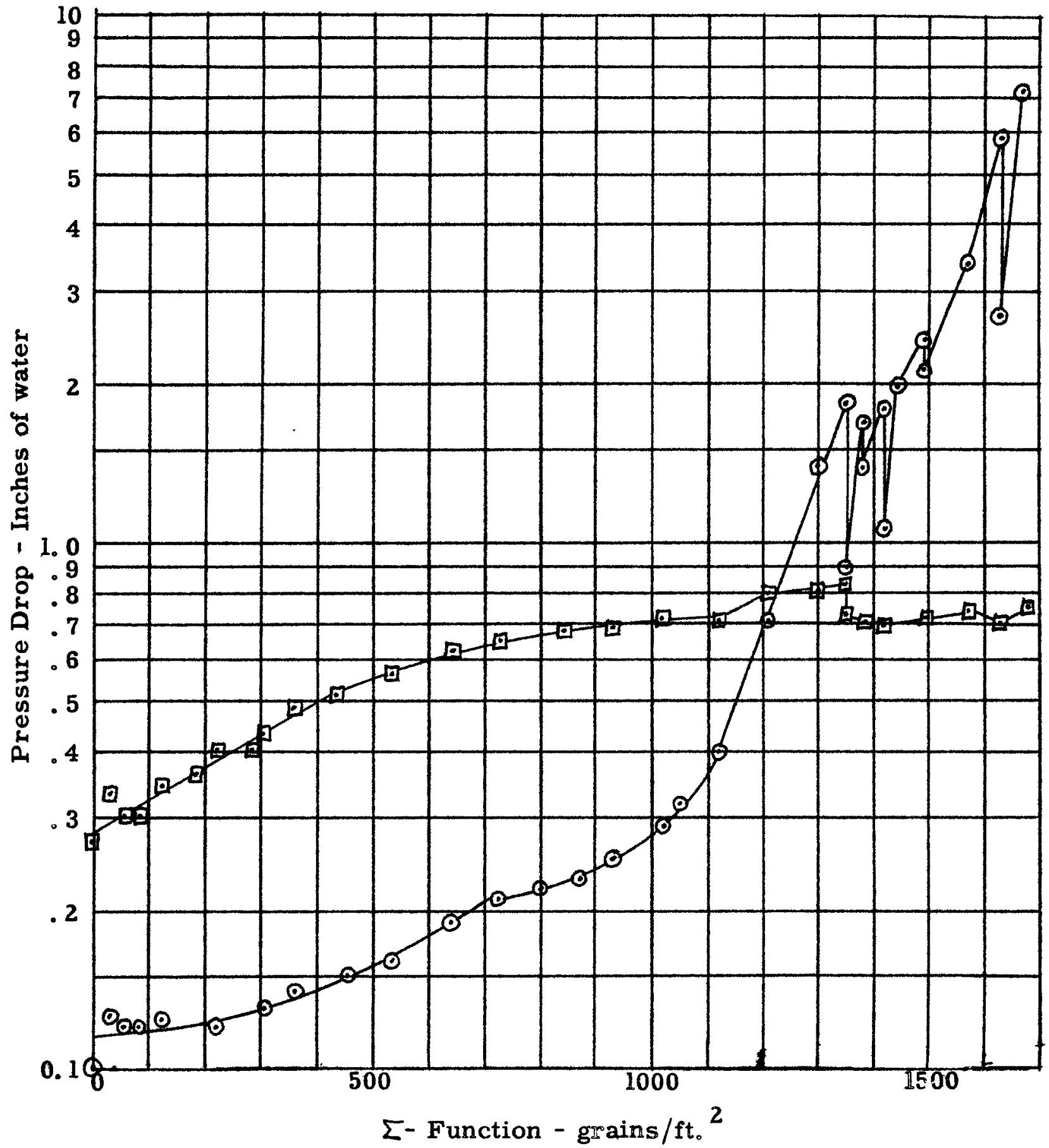




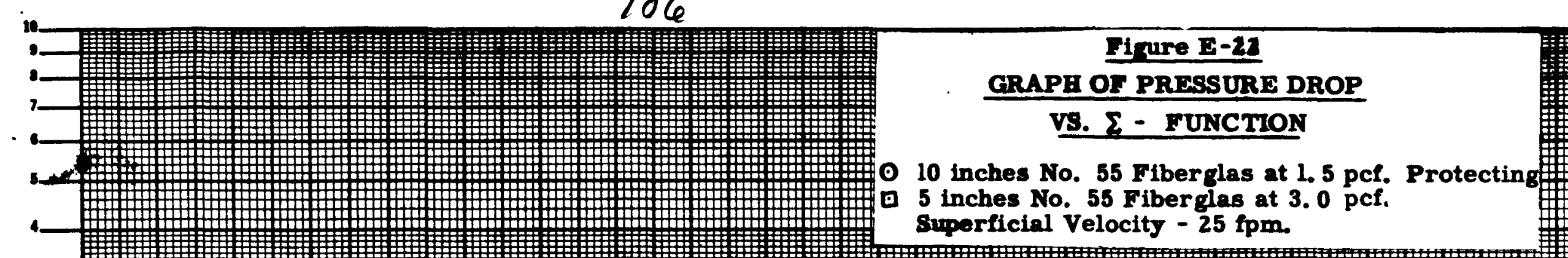

\section{GRAPH OF PRESSURE DROP \\ VS. $\Sigma$ - FUNCTION \\ 5 inches No. 55 Fiberglas at 3.0 pcf.}

姍

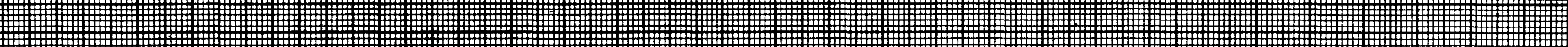

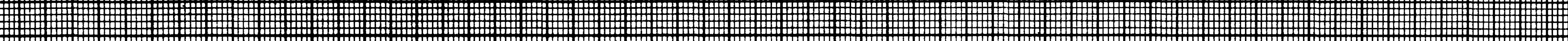

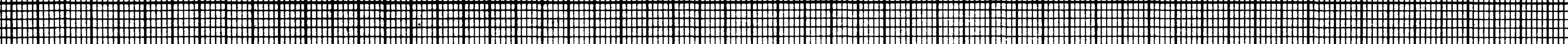

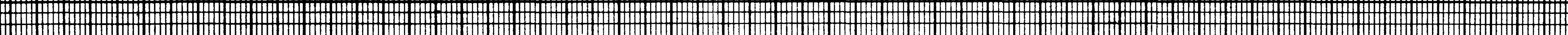

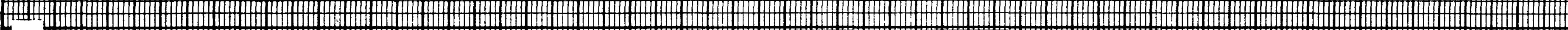
(1)

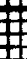

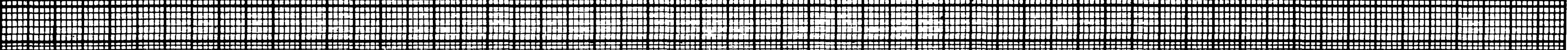

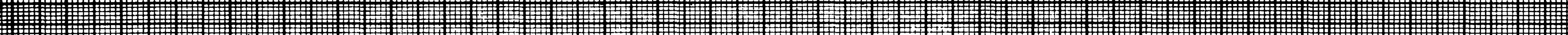

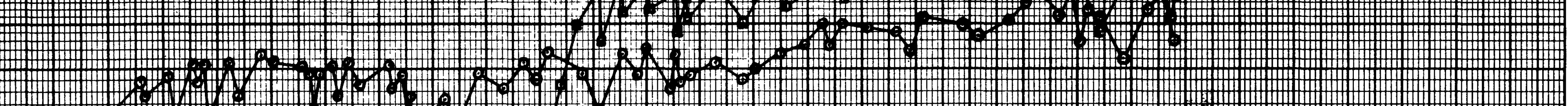

0

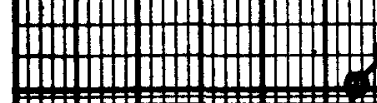

mis

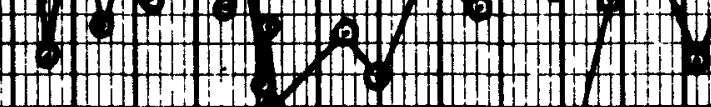

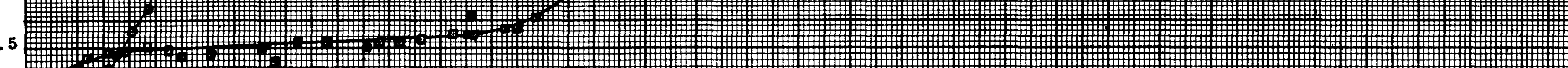

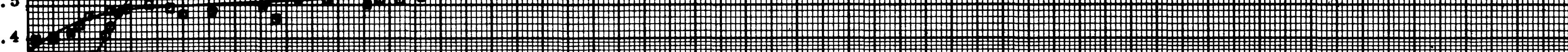

3.

|

2 鹿

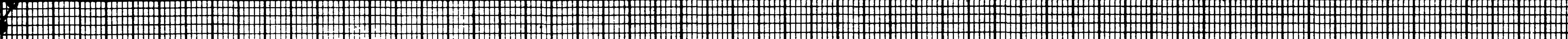

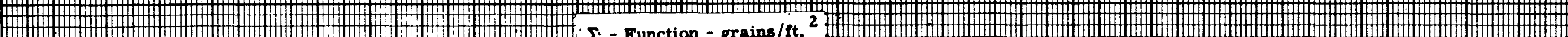

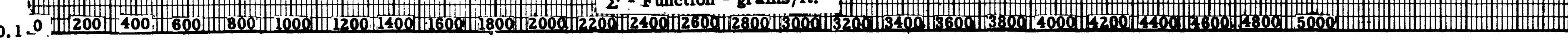


0 - 24 inches of No 450 Fiberglas at

2.0 pct. protecting.

- - 14. inches of No. 55 Fiberglas at 1.5 pef.

Superficial velocity $-25 \mathrm{fpm}$.

m
m M
ma

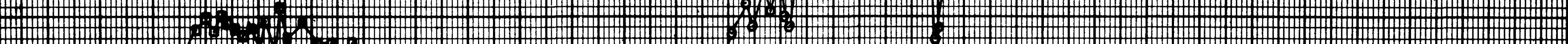

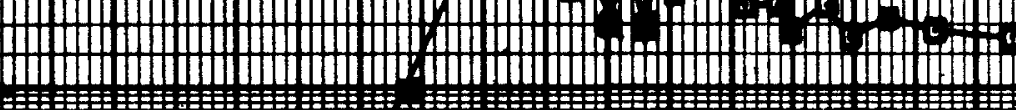

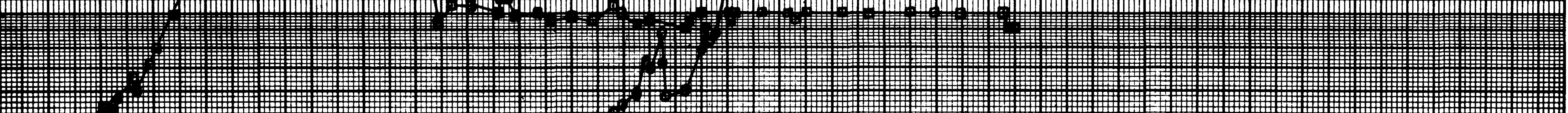

W
W (1)

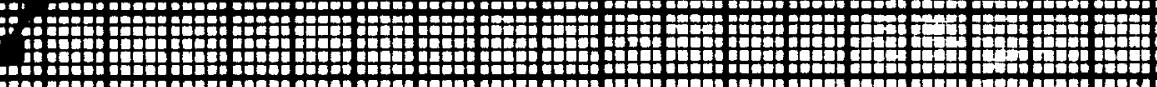

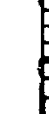

0.1 W
W I
I M
M a
na

.00

.00

.05

.04

.03 鹿耧

.02

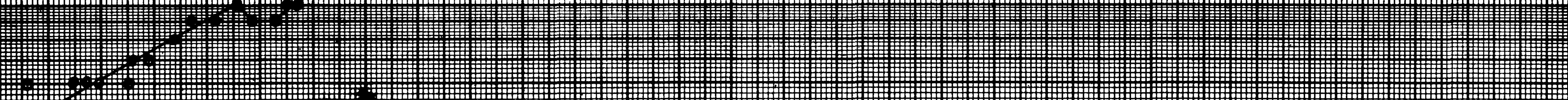

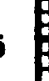

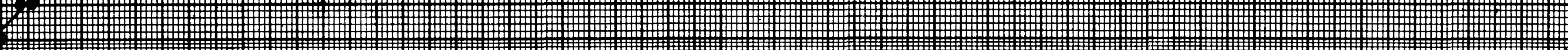

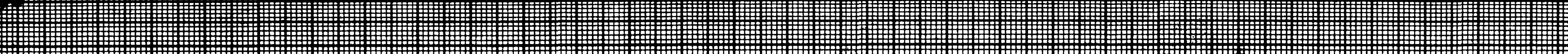
约 M
I 银

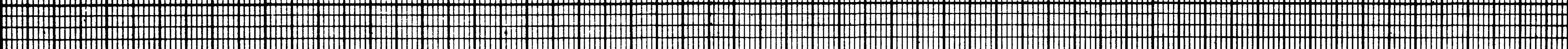

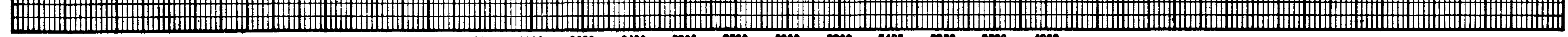


10

5

4

1.0

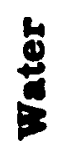

$\infty$

$\infty$

0.10

\section{Figure E-24}

$-103-$

GRAPH OF PRESSURE DROP VS. $\Sigma$ FUNCTION

- -24 inches of No. 800 Fiberglas at 5.0 pcf. Protecting

Q1- 14 inches of No. 55 Fiberglas at 1.5 pcf. Superficial Velocity - $25 \mathrm{fpm}$.

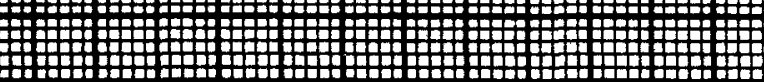

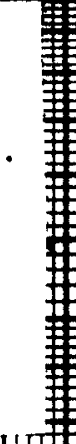

|

鹿 $\mid$
. | 约 (2) m
m

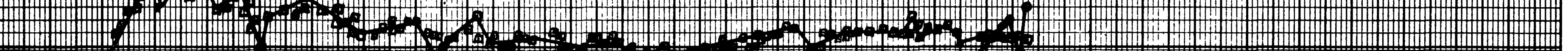

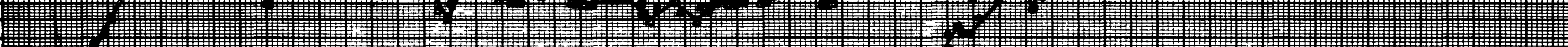
W W

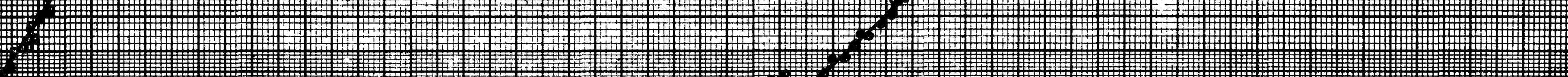

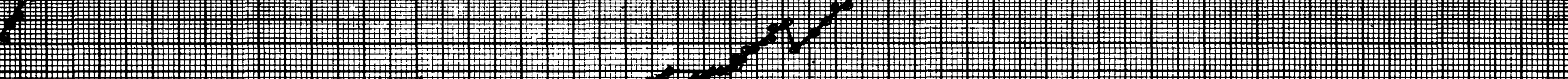
I
l 约 m
m

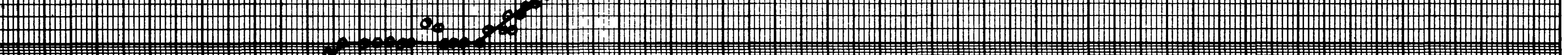

.04)

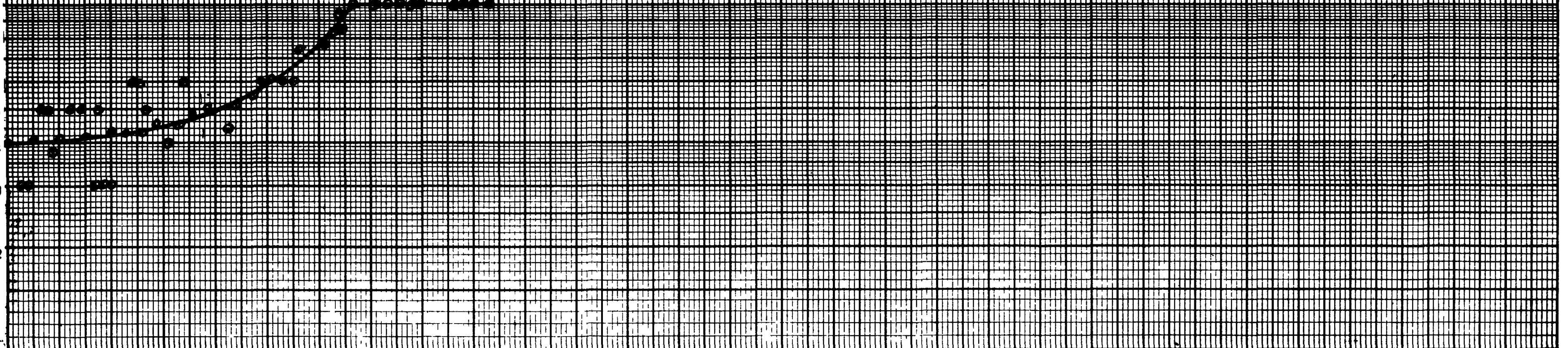

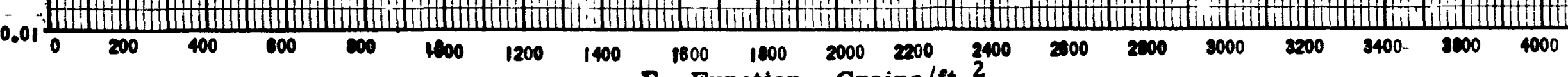
$\Sigma$-Function - Grains/ft. ${ }^{1000}$ 
100

c.

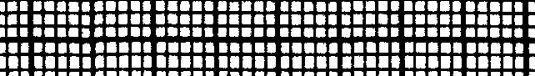

3
Figure E-25

GRAPH OF PRESSURE DROP VS. $\Sigma$ FUNCTION FOR A COMPOSITE UNIT OPERATED AT $50 \mathrm{fpm}$.

$1-16$ inches of No. 55 Fiberglas at 1.5 pcf. $1-16$ inches of No. 55 Fiberglas at 1.5 pcf.
$2-6$ inches of No. 55 Fiberglas at 3.0 pcf. $2-6$ inches of No. 55 Fiberglas at 3.0 pcf.
$3-8$ inches of No. 55 Fiberglas at 6.0 pcf. 4 - 1 inch of AA Fiberglas at 1.2 pcf. 5 - entire filter unit.
$1 / 3$

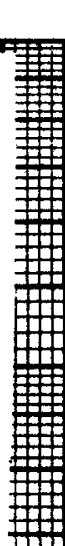

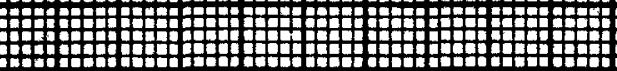
约

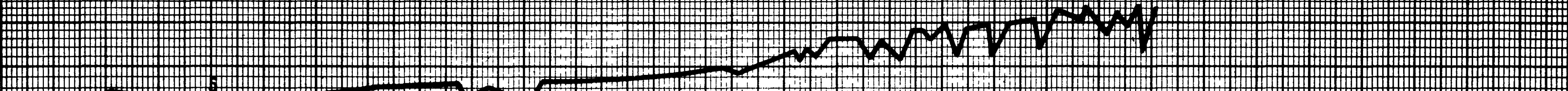

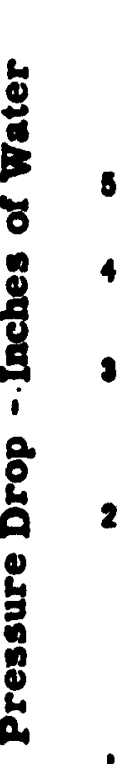

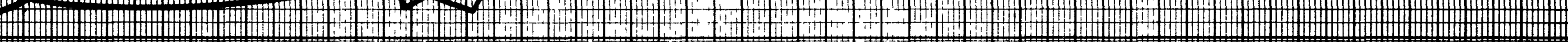

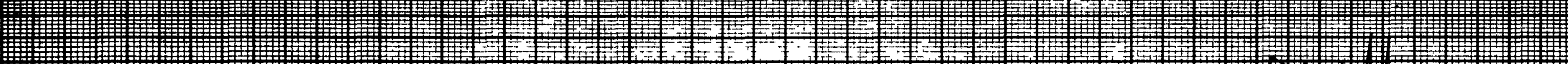

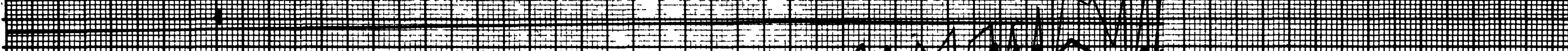

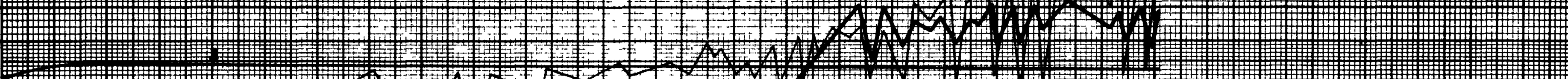

W

W W m m
m

1.0 (1)

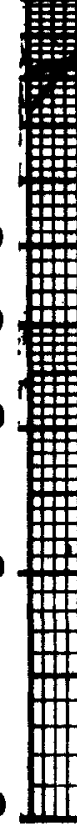

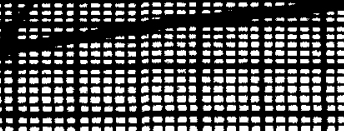

.000

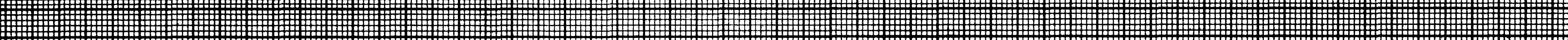

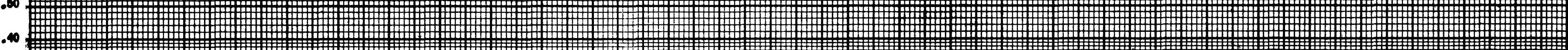

.20

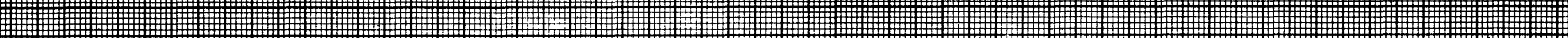
鹿 M
M

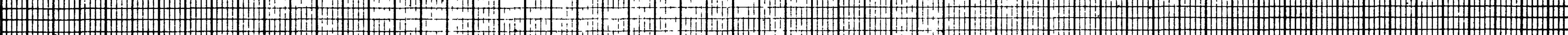

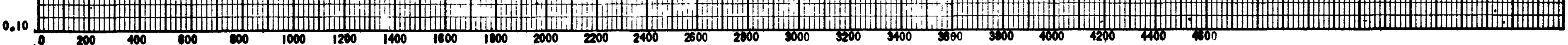
$\Sigma-$ Function-Grains/ft. 2 
10

.

3

2

1.0

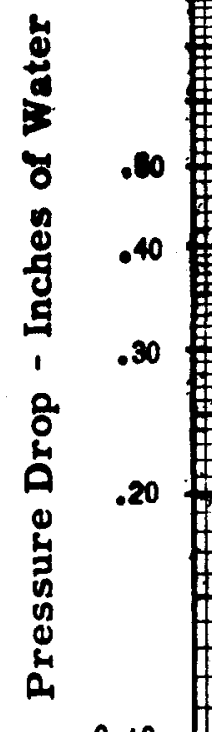

...

.04

.03

.02

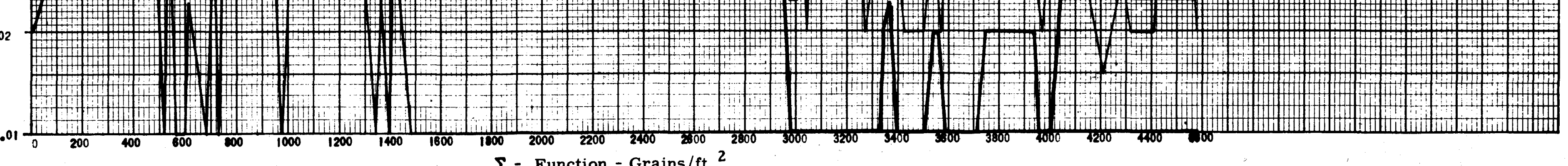

GRAPH OF PRESSURE DROP VS. $\Sigma$ FUNCTION FOR A COMPOSITE

TEST UNIT OPERATED AT $25 \mathrm{fpm}$.

1 - 1st. inch of No. 55 Fiberglas at 1.5 pcf.

3 - lst inch of No. 55 Fiberglas at 3.0 pcf.
Figure E-26A

2 - 2nd and 3rd inches of No. 55 Fiberglas at 1.5 pcf. 约 M

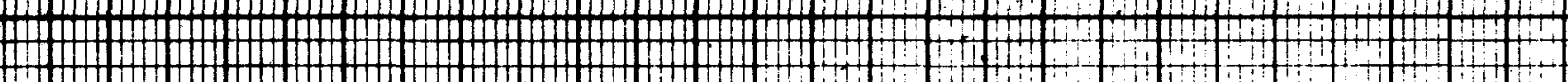
m

(1)

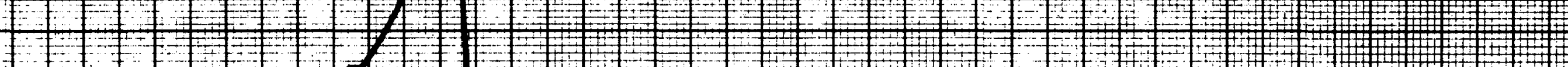
W

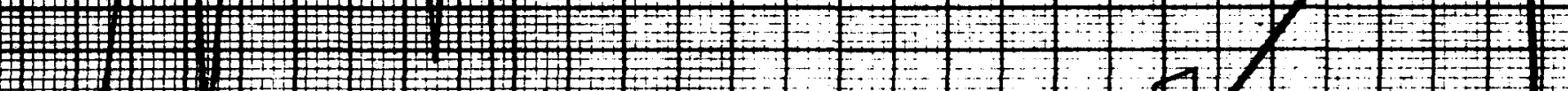

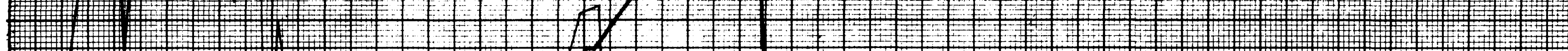

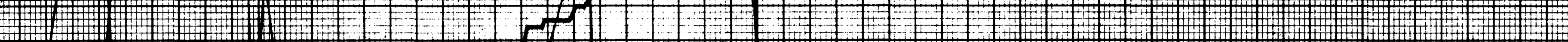

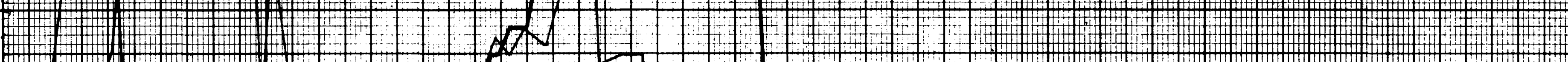
m m m m m m

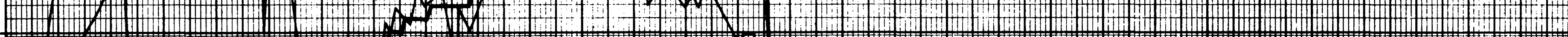

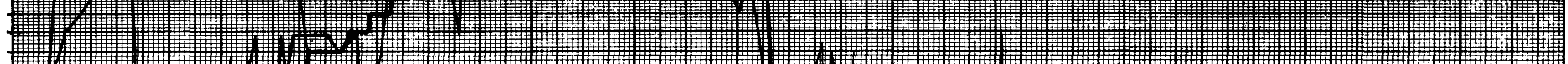
J
J J (W) J
J \# \# 
$1 / 6$

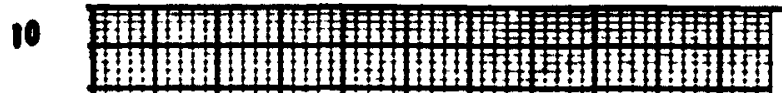

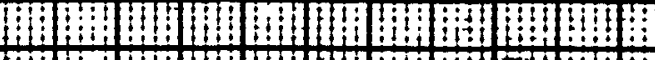

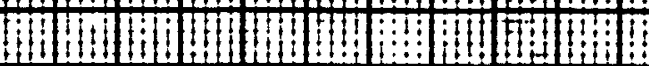

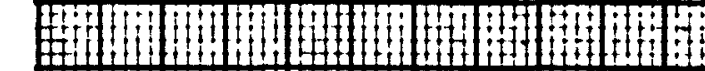

3

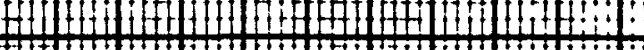

4

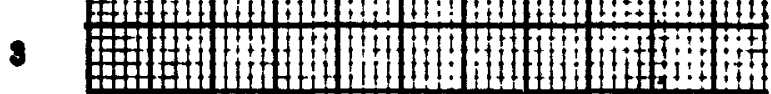
$(1)+(-1)$
Figure E-26 B

GRAPH OF PRESSURE DROP VS. $\Sigma$ FUNCTION FOR A COMPOSITE TEST UNIT OPERATED AT 25 fpm

$1-14$ inches of No. 55 Fiberglas at 1.5 pcf 2 - 6 inches of No. 55 Fiberglas at 3.0 pcf. 3 - 8 inches of No. 55 Fiberglas at 6.0 pct 5 - entire filter of $\mathrm{AA}$

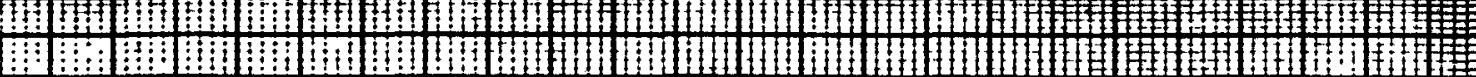
: 7.1. L. †十 1.7.7.

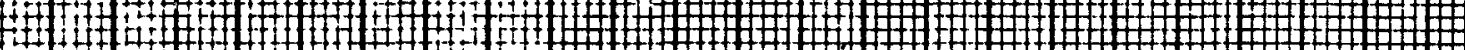

1.0

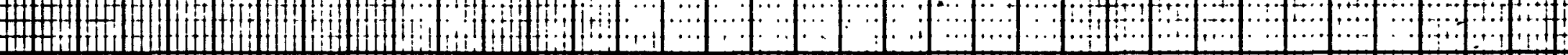
(1)

0.1

$\because$

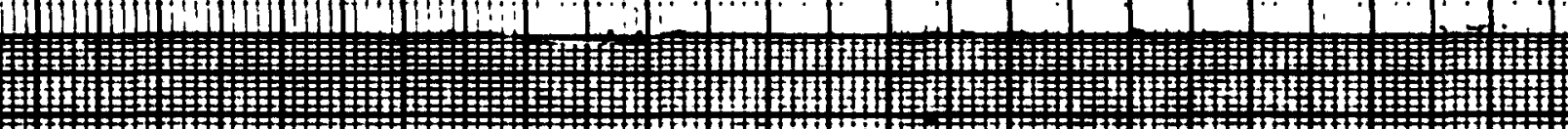

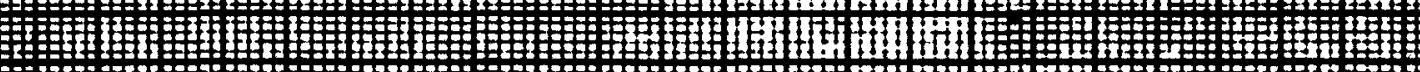

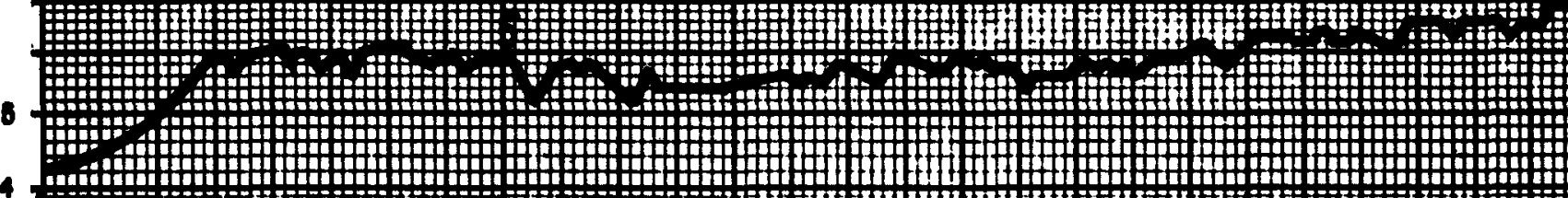

'.

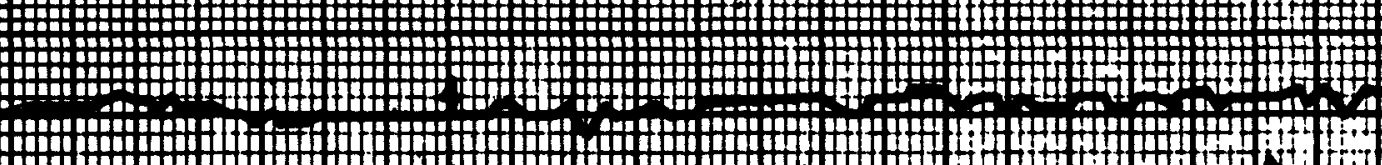

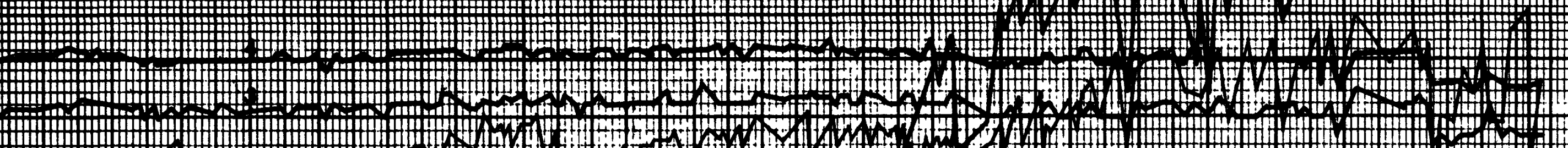

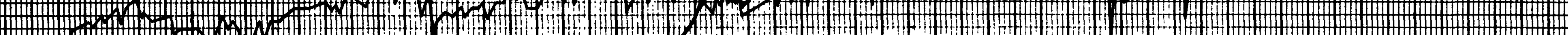

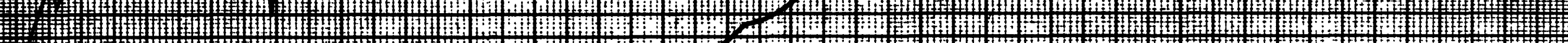
(2)

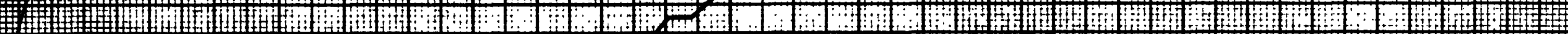

.05

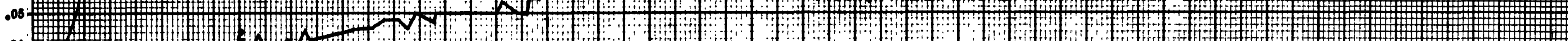

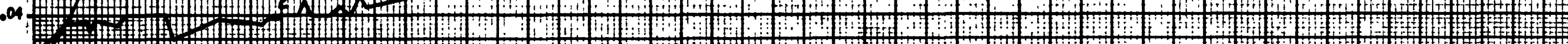

.00

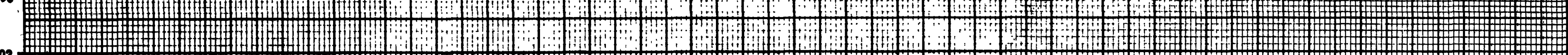

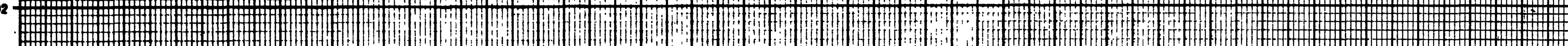

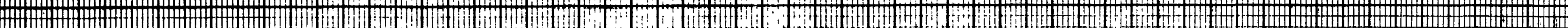

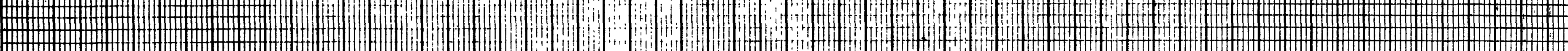

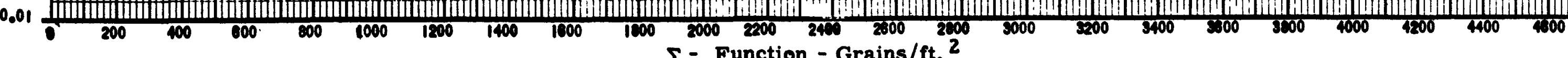


Figure E-27

GRAPH OF PRESSURE DROP VS. $\sum$ FUNCTION

๑-8 inches of No. 55P Fiberglas at 6.0 pcf protecting

D-0.75 inch of AA Fiberglas at 1.2 pcf.

Superficial Velocity - 25 fpm.

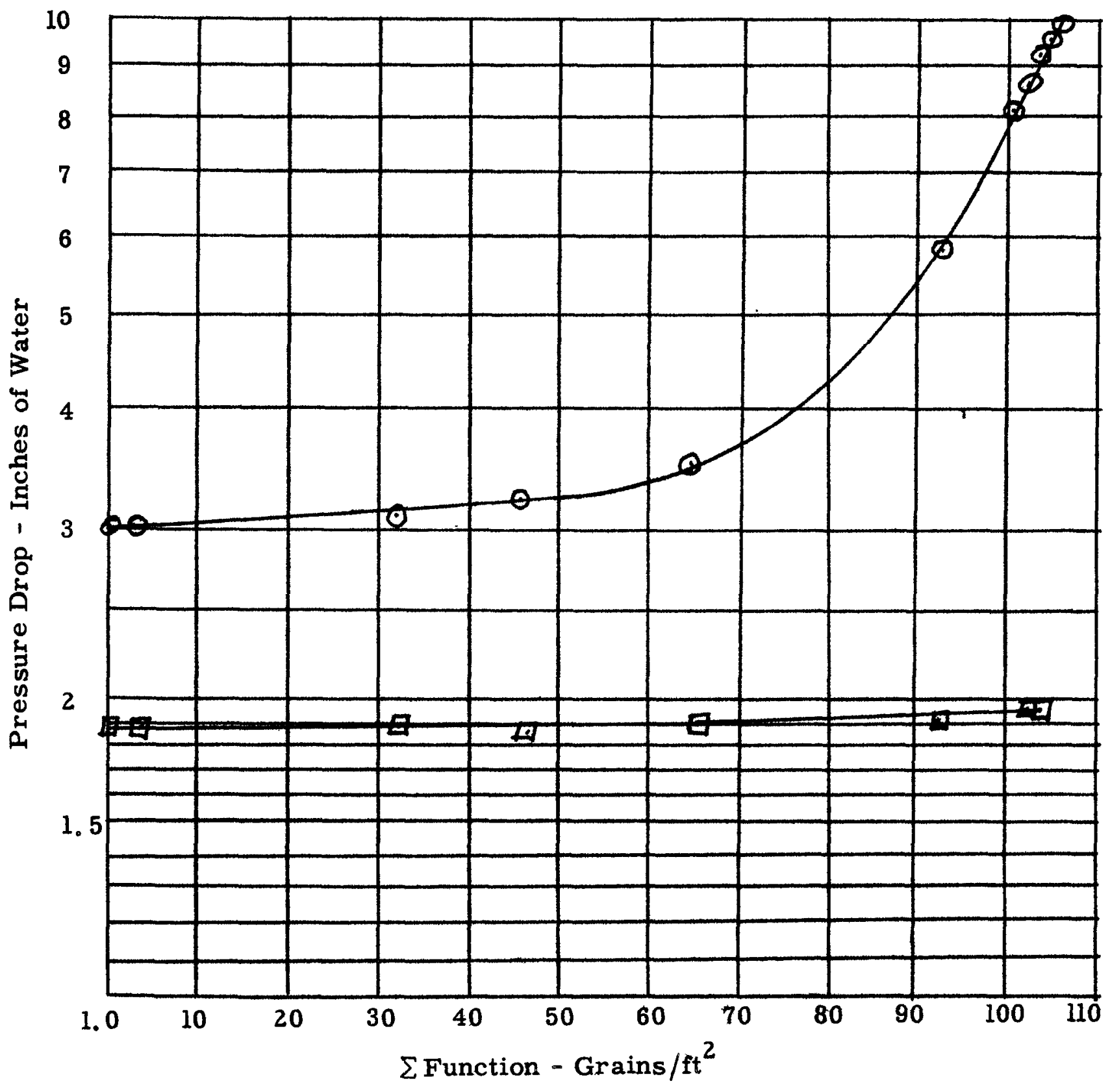


Figure E-28

GRAPH OF PRESSURE DROP VS. $\sum$ FUNCTION

0-6 inches No, 55P Fiberglas at 3.0 pcf. protecting

๑- 8 inches No, 55P Fiberglas at 6.0 pcf。

Superficial Velocity - $25 \mathrm{fpm}$.

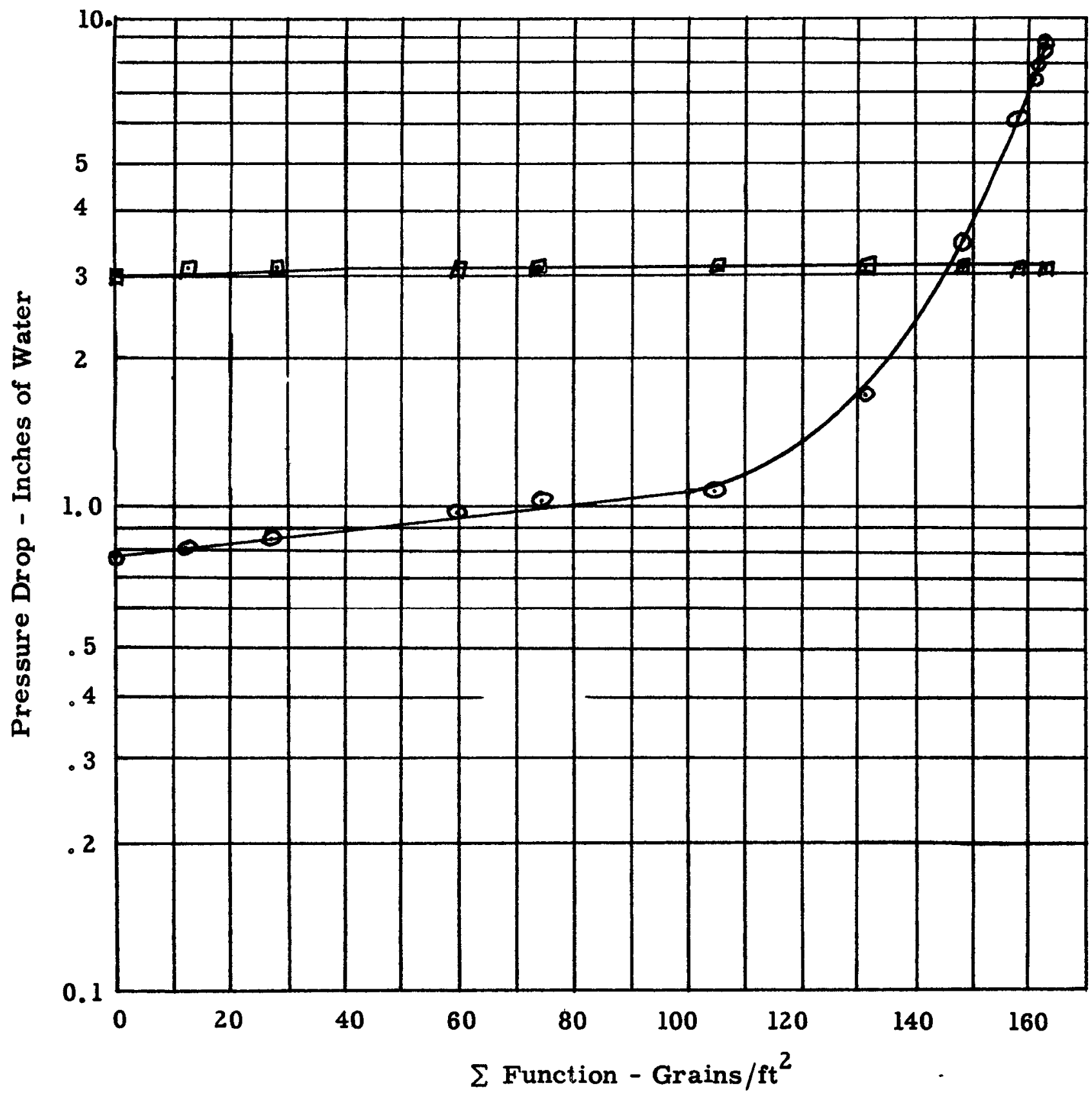




\section{Figure E-29 \\ GRAPH OF PRESSURE DROP VS. $\sum$ FUNCTION}

-18 inches of No. 55P Fiberglas at 1.5 pfc. protecting

[-8 inches of No. 55P Fiberglas at 3.0 pcf.

Superficial Velocity - 25 fpm.

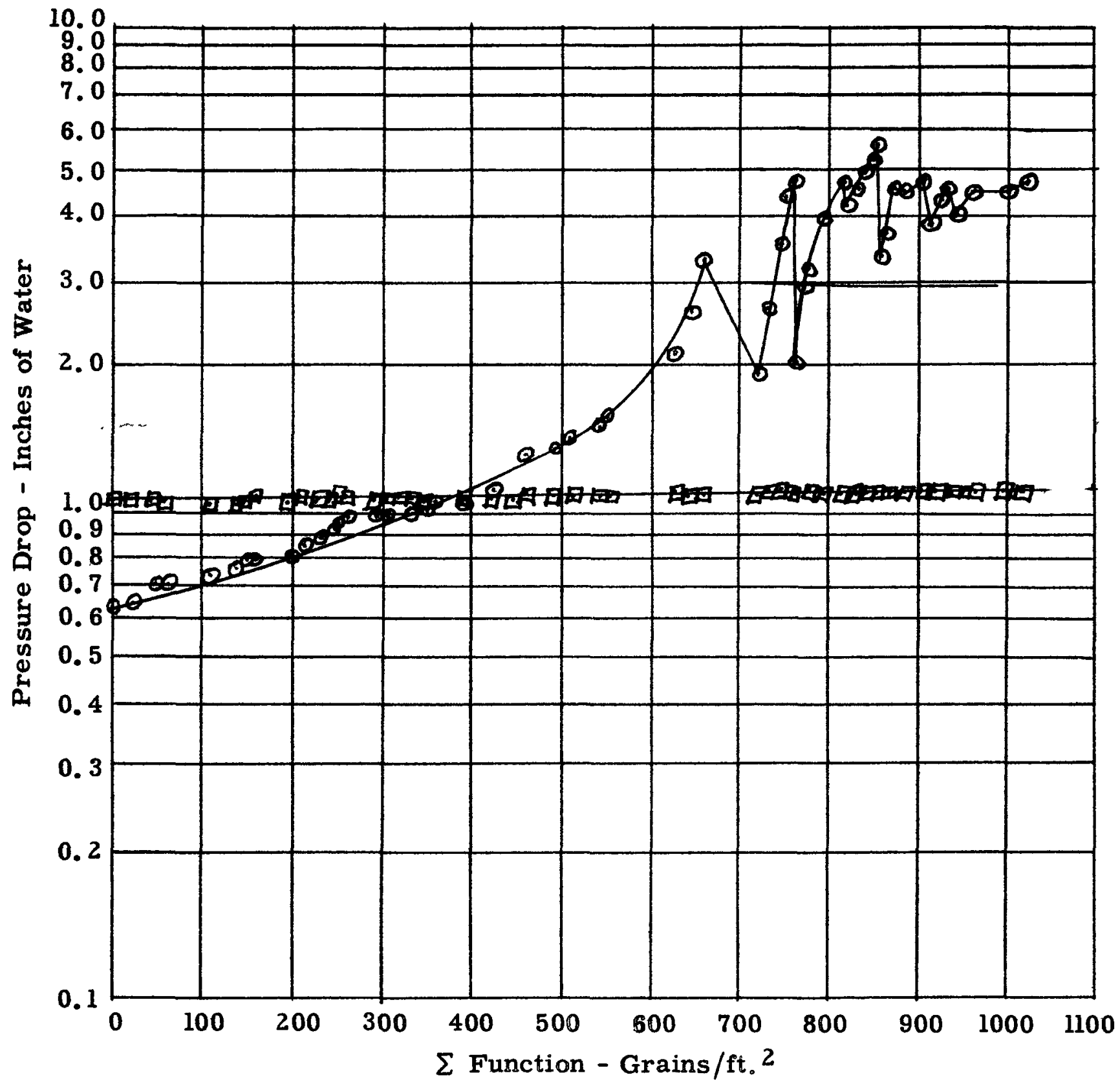


Figure E-30

\section{1}

GRAPH OF PRESSURE DROP VS. $\Sigma$ FUNCTION

0- 8 inches of No. 115K Fiberglas at 9.0 pcf. protecting.

口- 3/4 inch AA Fiberglas at 1.2 pcf. Superficial Velocity - 50 fpm.

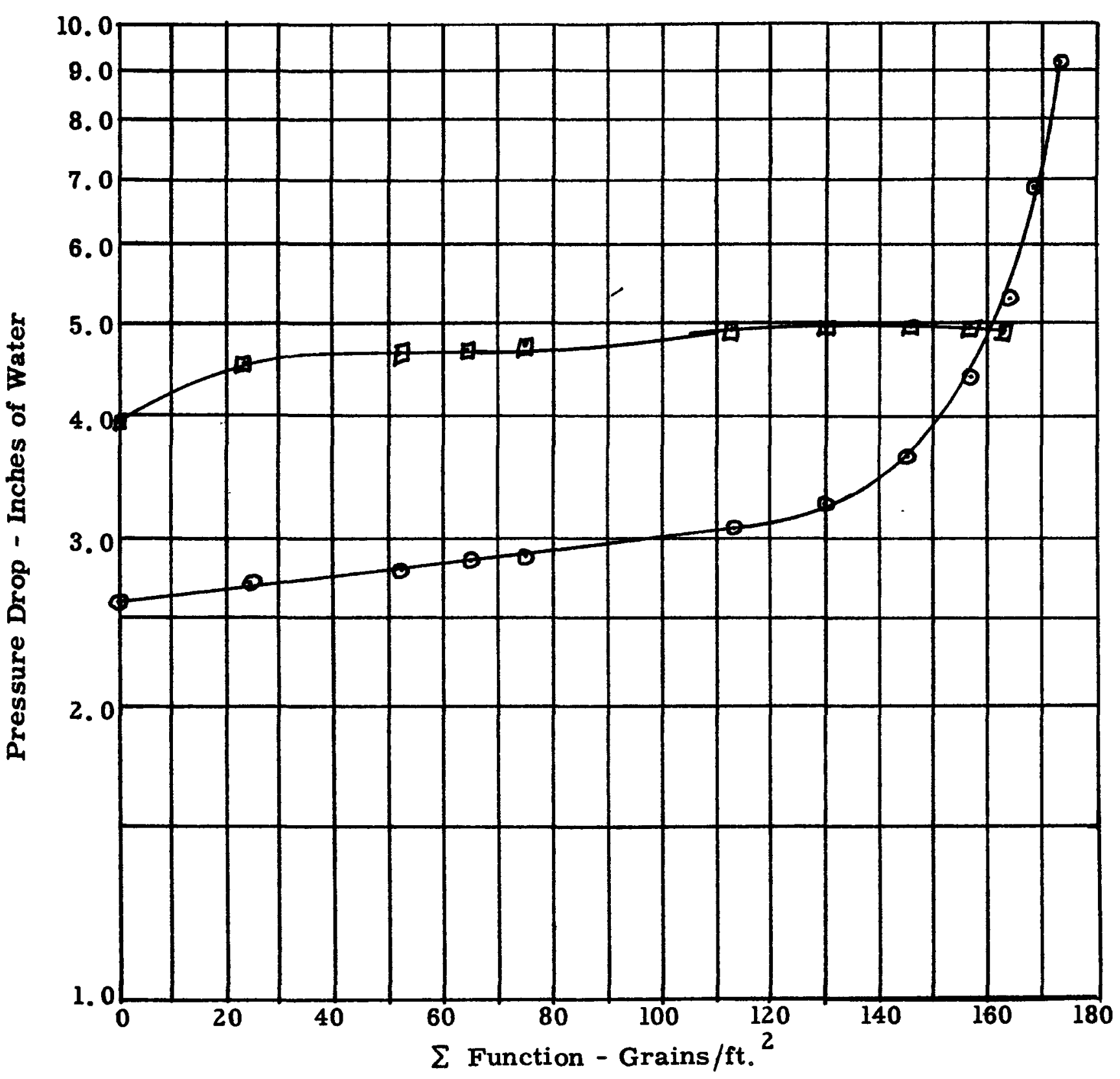


Figure $E-31$

\section{GRAPH OF PRESSURE DROP VS。 $\Sigma$ FUNCTION}

口- 6 inches of No. 115K Fiberglas at 6.0 pcf. protecting.

A- 8 inches of No. 115K Fiberglas at 9.0 pcf.

$\odot-1$ st inch of No. $115 \mathrm{~K}$ Fiberglas at 6.0 pcf.

Superficial Velocity - 50 fpm.

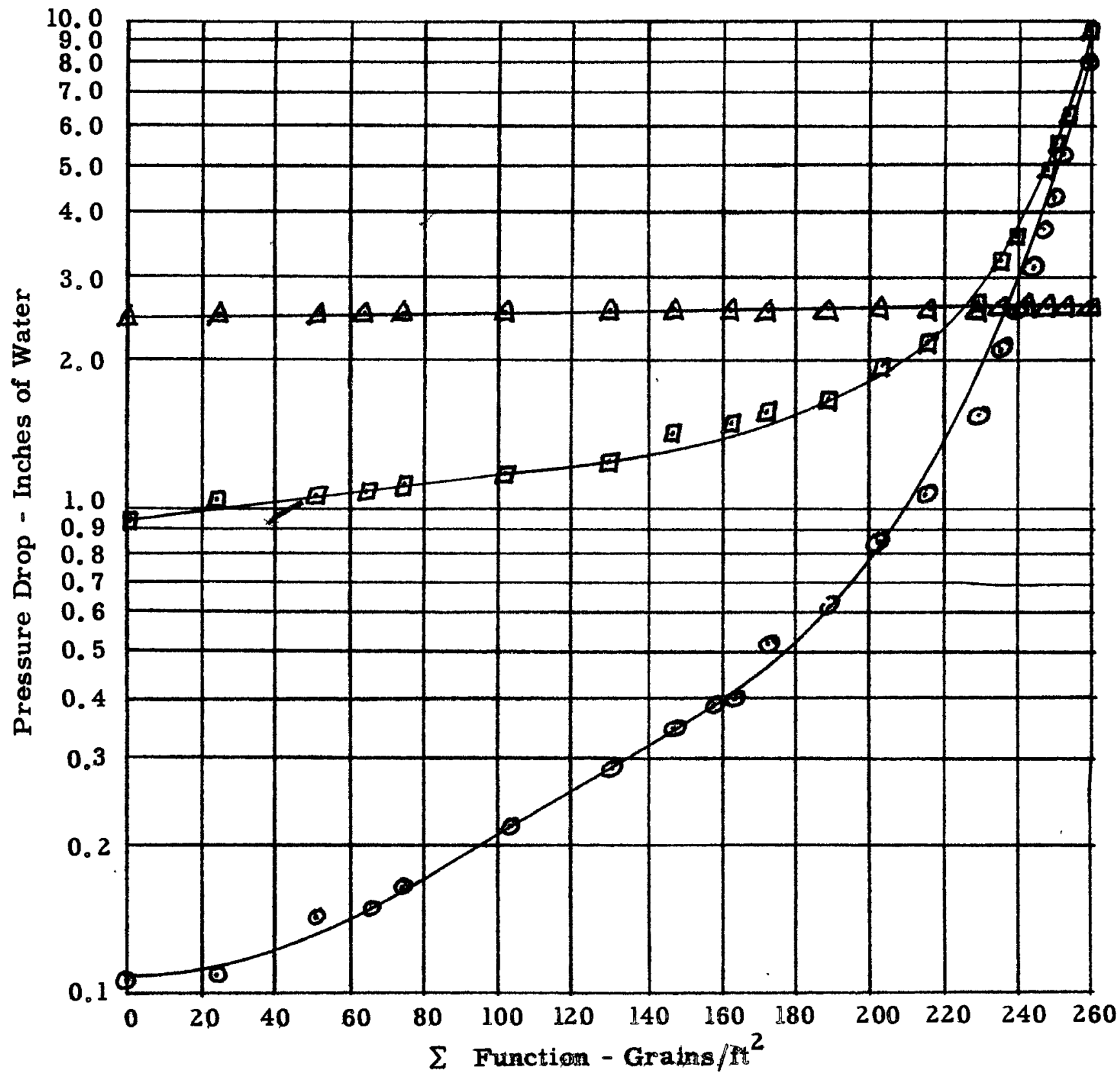




$$
-112-
$$

Figure E-32

GRAPH OF PRESSURE DROP VS. $\sum$ FUNCTION

0- 6 inches of No. 115K Fiberglas at 3.0 pcf. protecting.

曰- 8 inches of No. $115 \mathrm{~K}$ Fiberglas at 6.0 pcf. Superficial Velocity - 50 fpm.

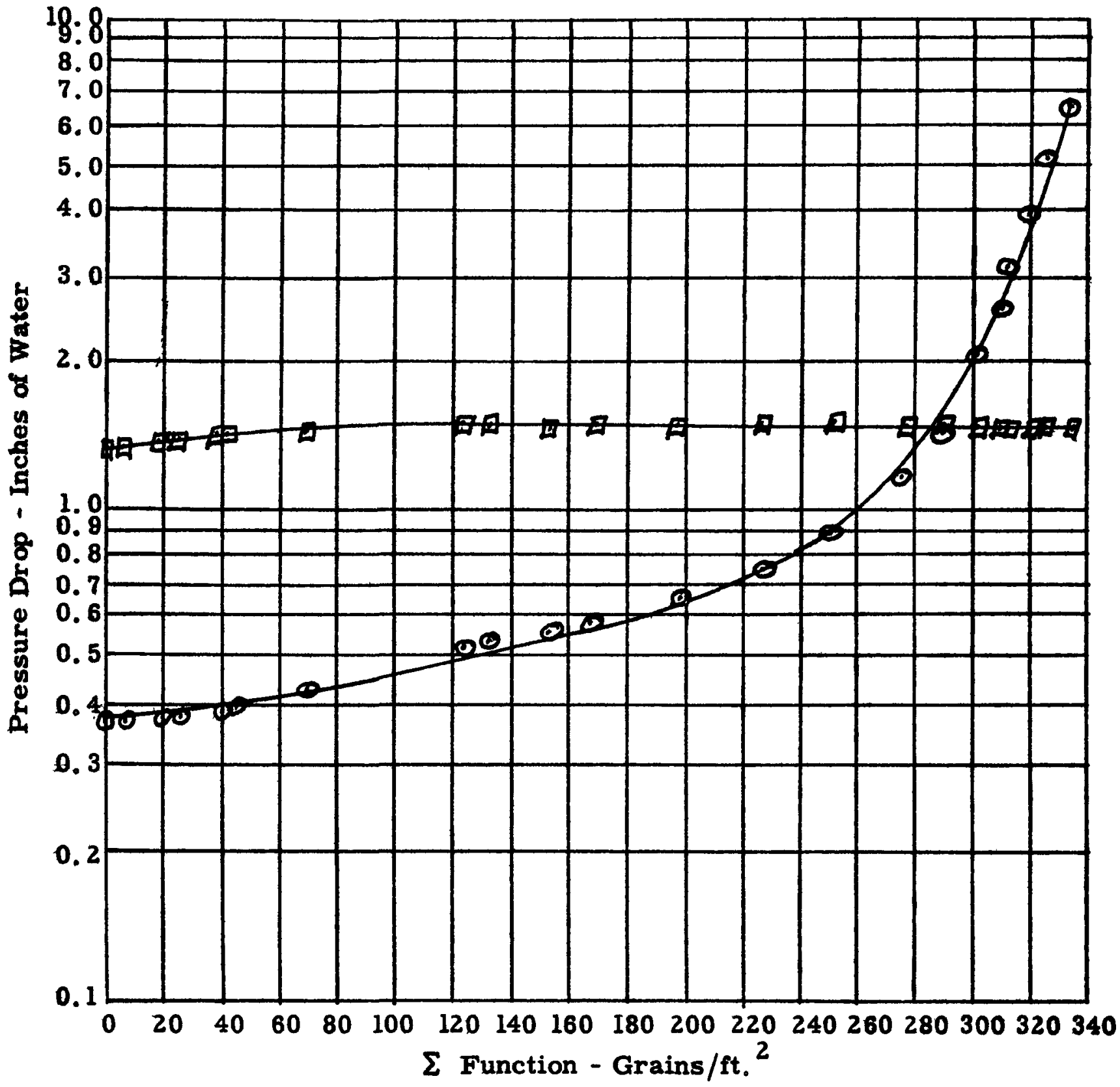




\section{Figure E-33 124}

GRAPH OF PRESSURE DROP VS. $\Sigma$ FUNCTION

$\odot-12$ inches No. 115K Fiberglas at 1.5 pcf. protecting.

曰- 6 inches No. 115K Fiberglas at 3.0 pcf.

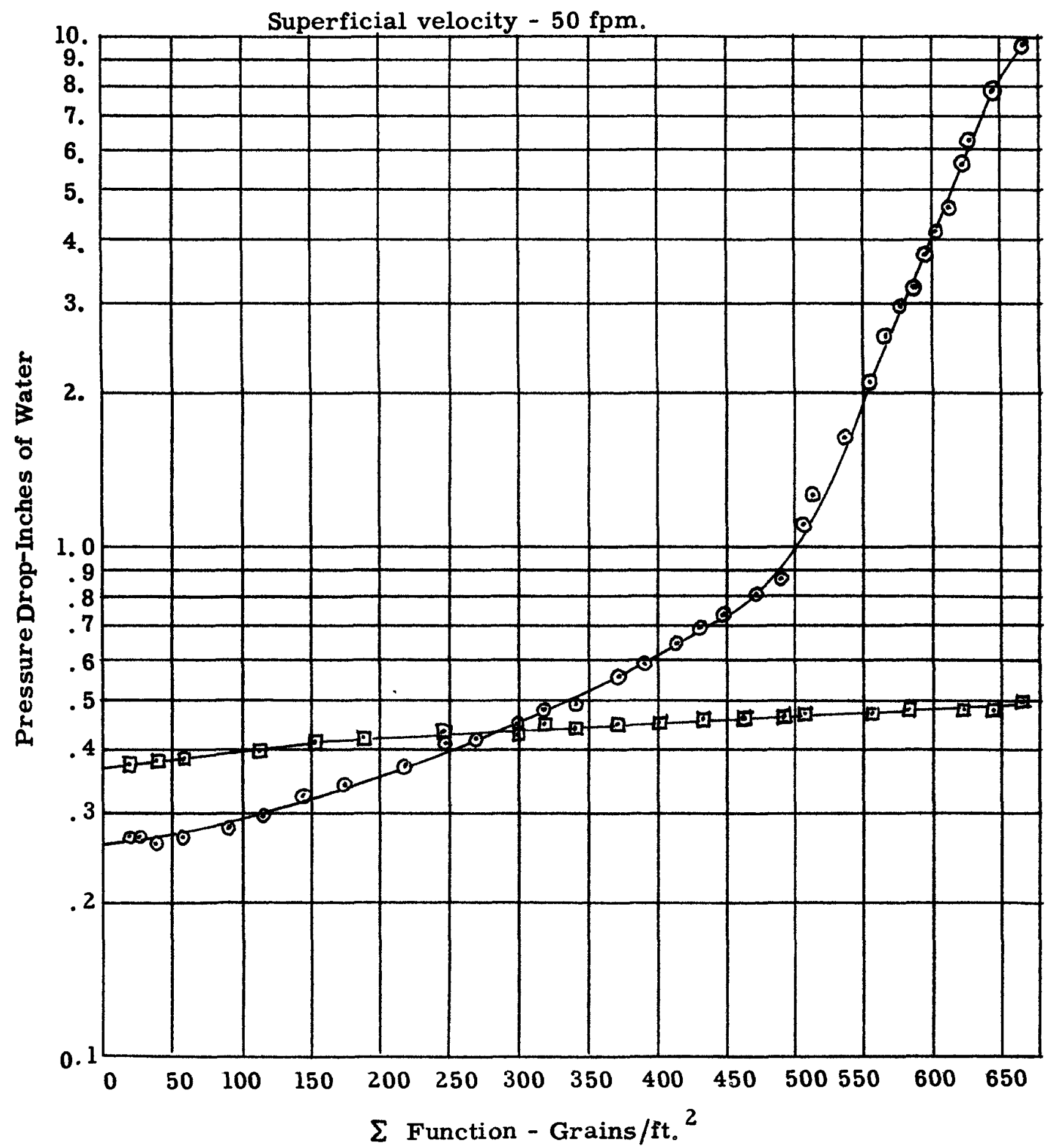




\section{Figure E-34 125}

\section{GRAPH OF PRESSURE DROP VS. $\Sigma$ FUNCTION}

○ - 8 inches No. 115K Fiberglas at 9.0 pcf. protecting.

घ-3/4 inch AA Fiberglas at 1.2 pcf. Superficial Velocity - 25 fpm.

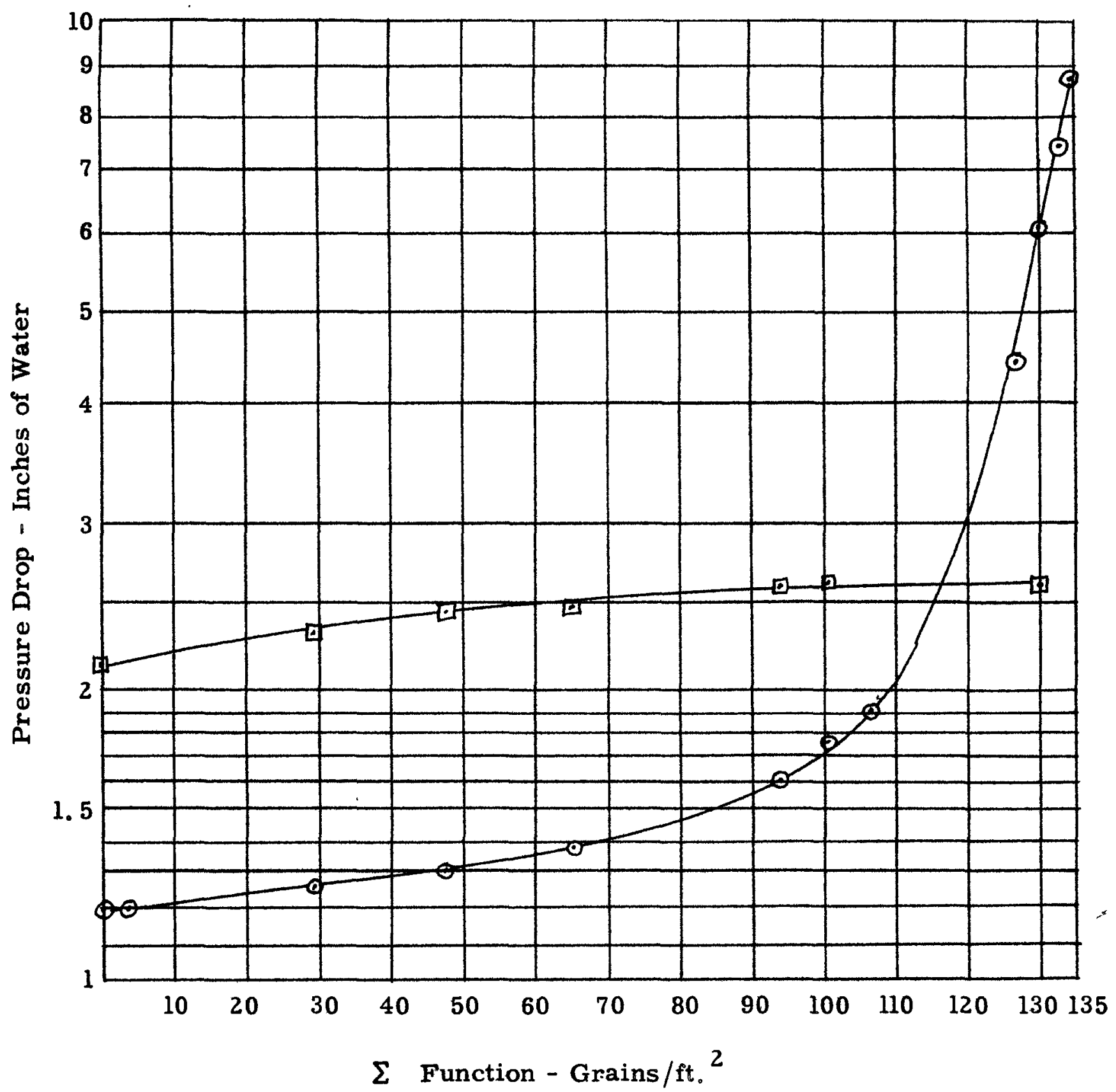




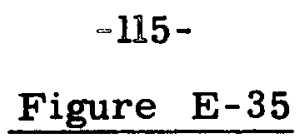

124

HW-20847

GRAPH OF PRESSURE DROP VS. $\Sigma$ FUNCTION

$\odot-6$ inches No. 115K Fiberglas at 6.0 pcf. protecting.

口- 8 inches No. 115K Fiberglas at 9.0 pcf.

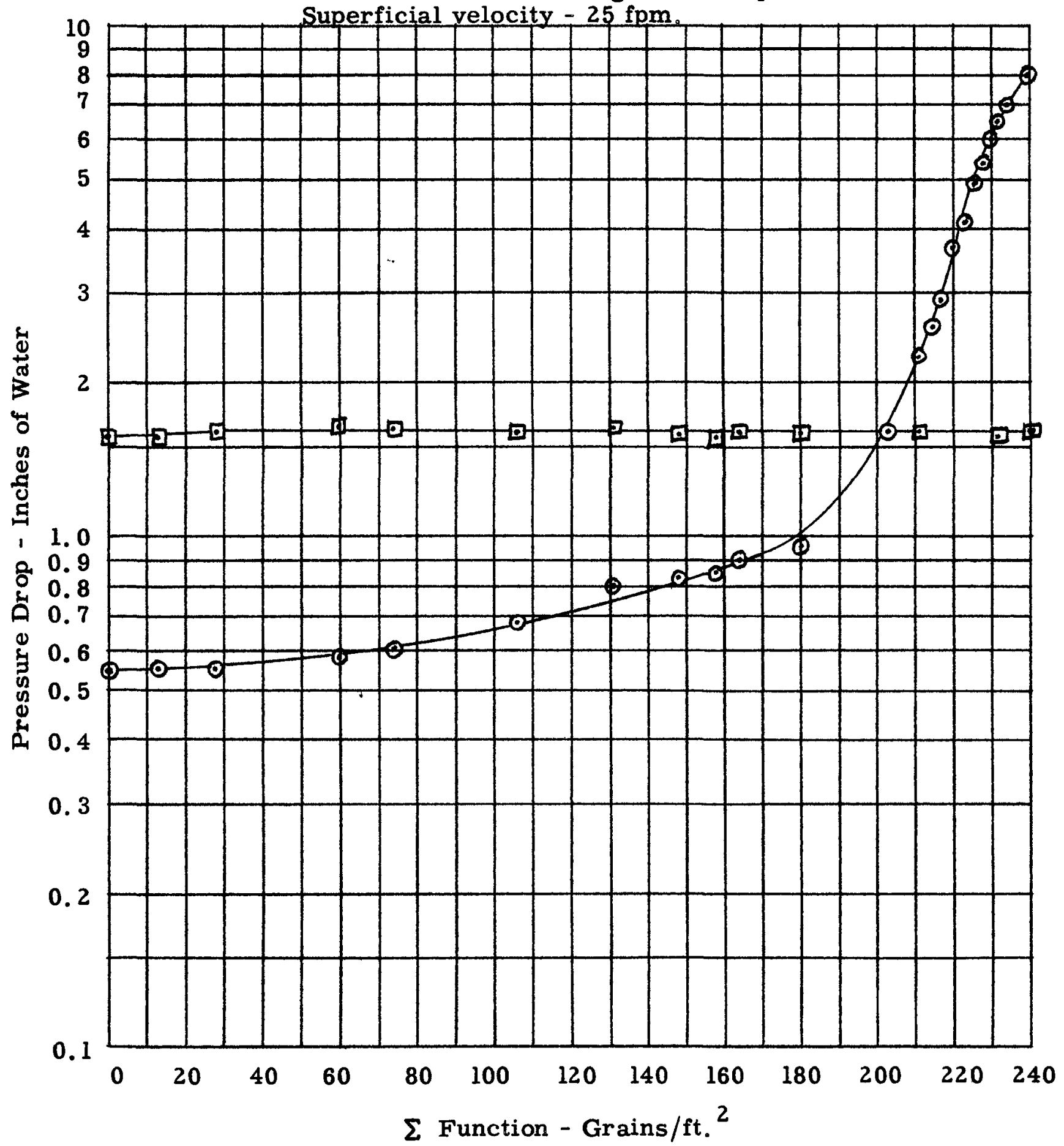




$$
-116-
$$

Figure E-36

GRAPH OF PRESSURE DROP VS. $\Sigma$ FUNCTION

6 inches No. 115K Fiberglas at 6.0 pcf.

Superficial Velocity - $25 \mathrm{fpm}$.

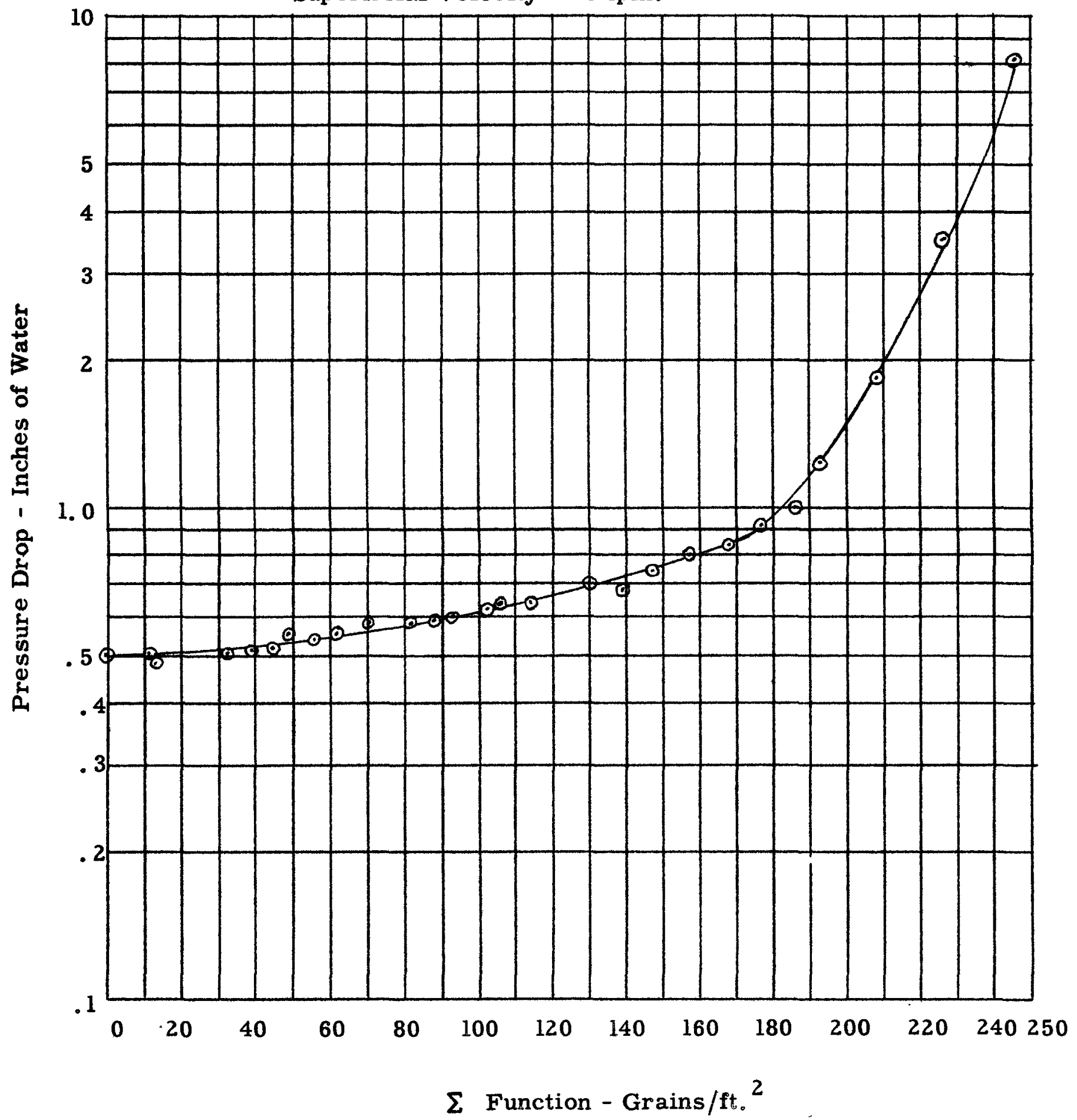


Figure E-37

GRAPH OF PRESSURE DROP VS. $\Sigma$ FUNCTION

$\odot-6$ inches No. 115K Fiberglas at 3.0 pcf. protecting.

口- 8 inches No. 115K Fiberglas at 6.0 pcf. Superficial Velocity - 25 fpm.

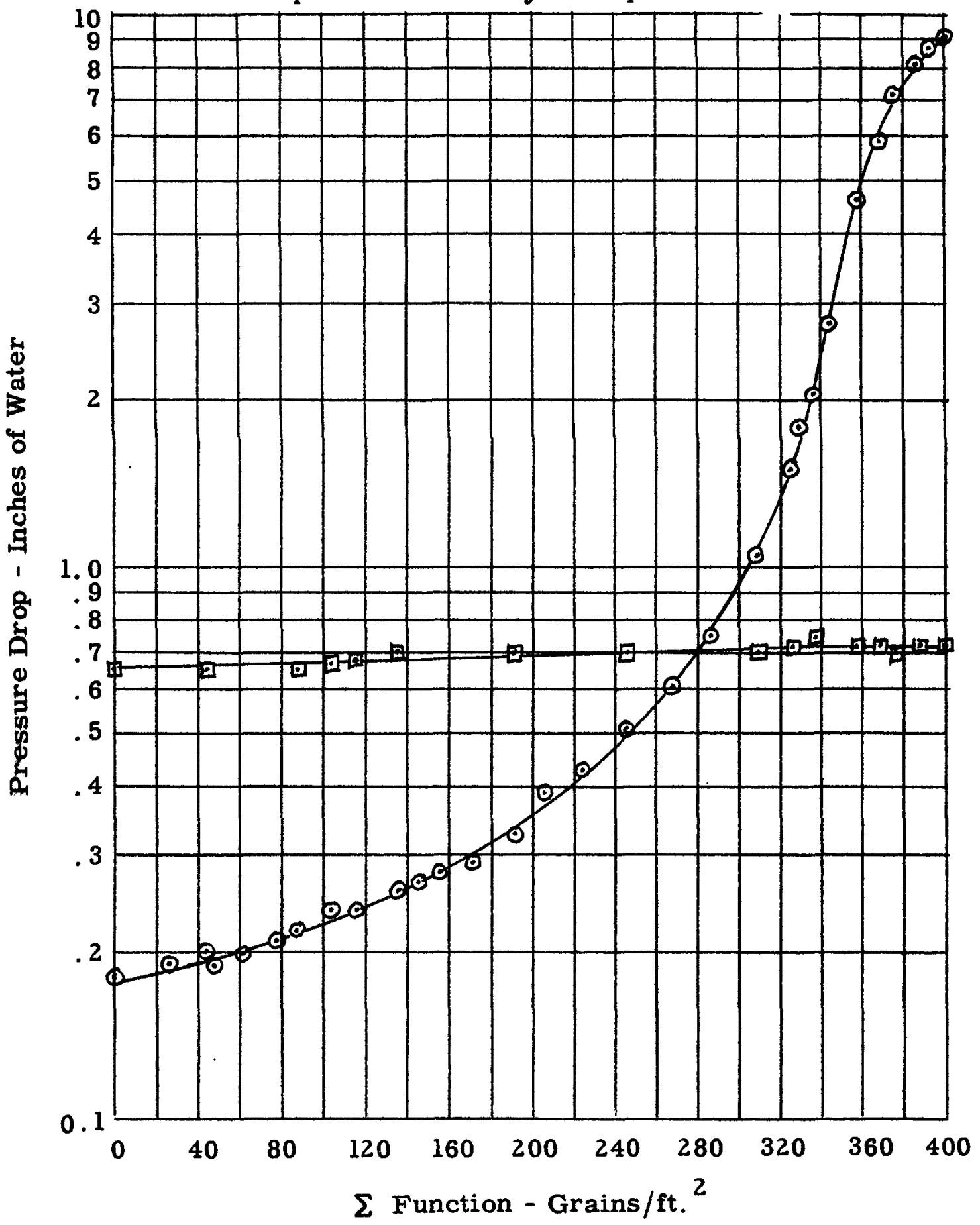




$$
\text { -118- } \quad 129 \quad \text { HW-20847 }
$$

Figure E-38

GRAPH OF PRESSURE DROP VS. $\Sigma$ FUNCTION

$\odot-6$ inches No. 115K Fiberglas at 3.0 pcf. protecting.

$\square-8$ inches No. 115K Fiberglas at 6.0 pcf。 Superficial Velocity - 25 fpm.

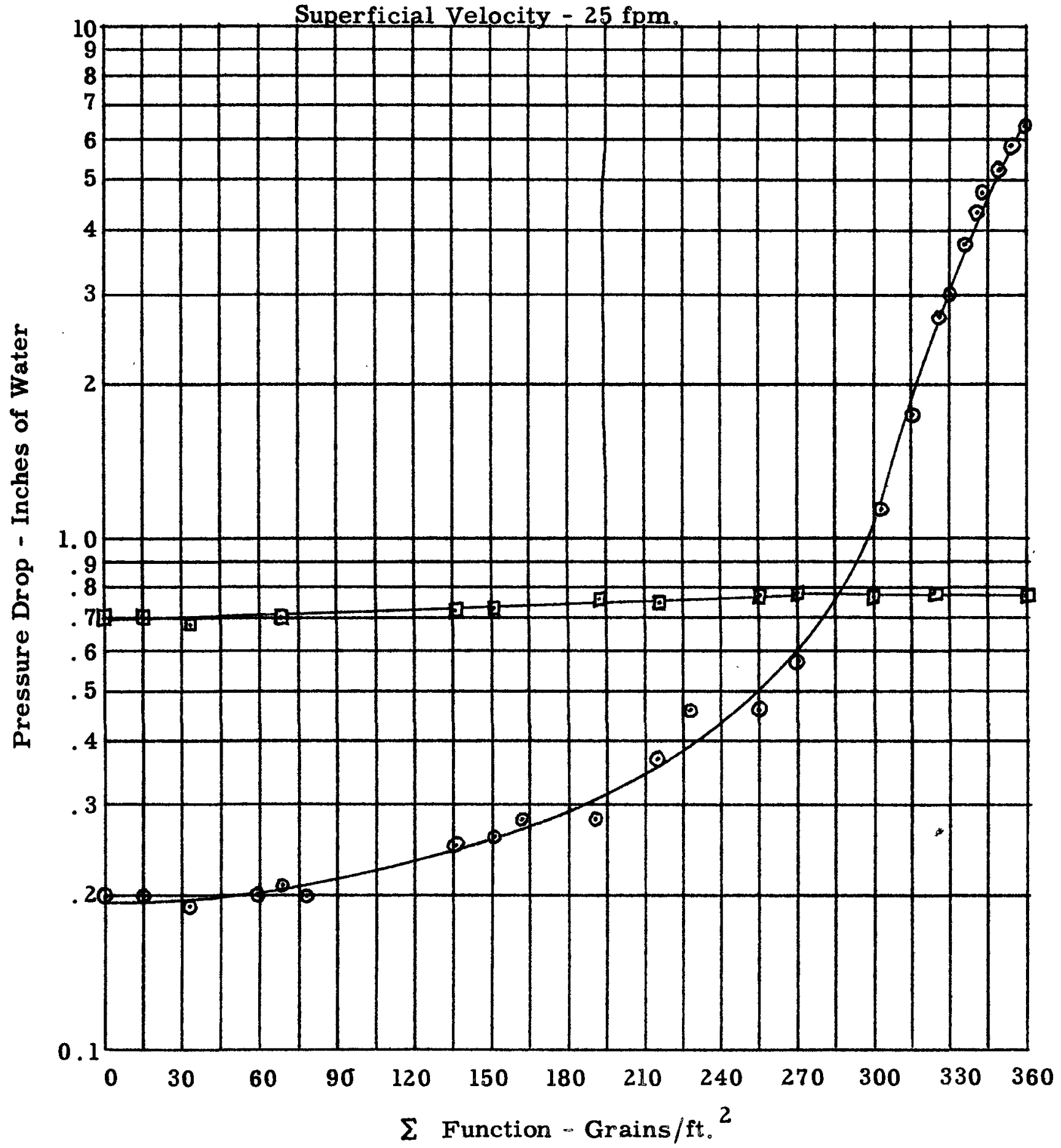


Figure E-39

GRAPH OF PRESSURE DROP VS. $\sum$ FUNCTION

$\Theta .-3$ inches No. 115K Fiberglas at 3.0 pcf. protecting.

口 - 6 inches No. $115 \mathrm{~K}$ Fiberglas at 6.0 pcf. Superficial Velocity - 15 fpm.

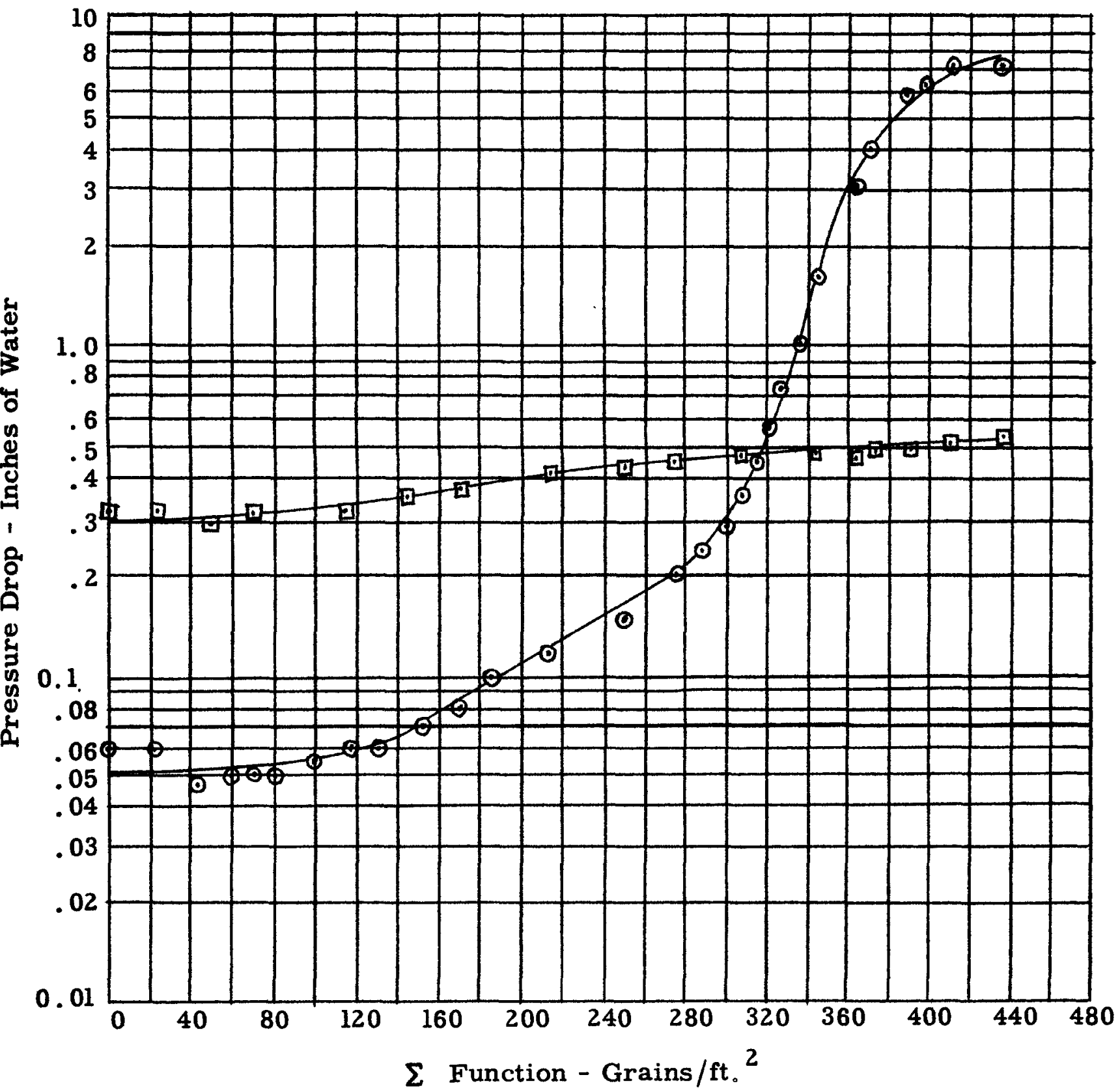




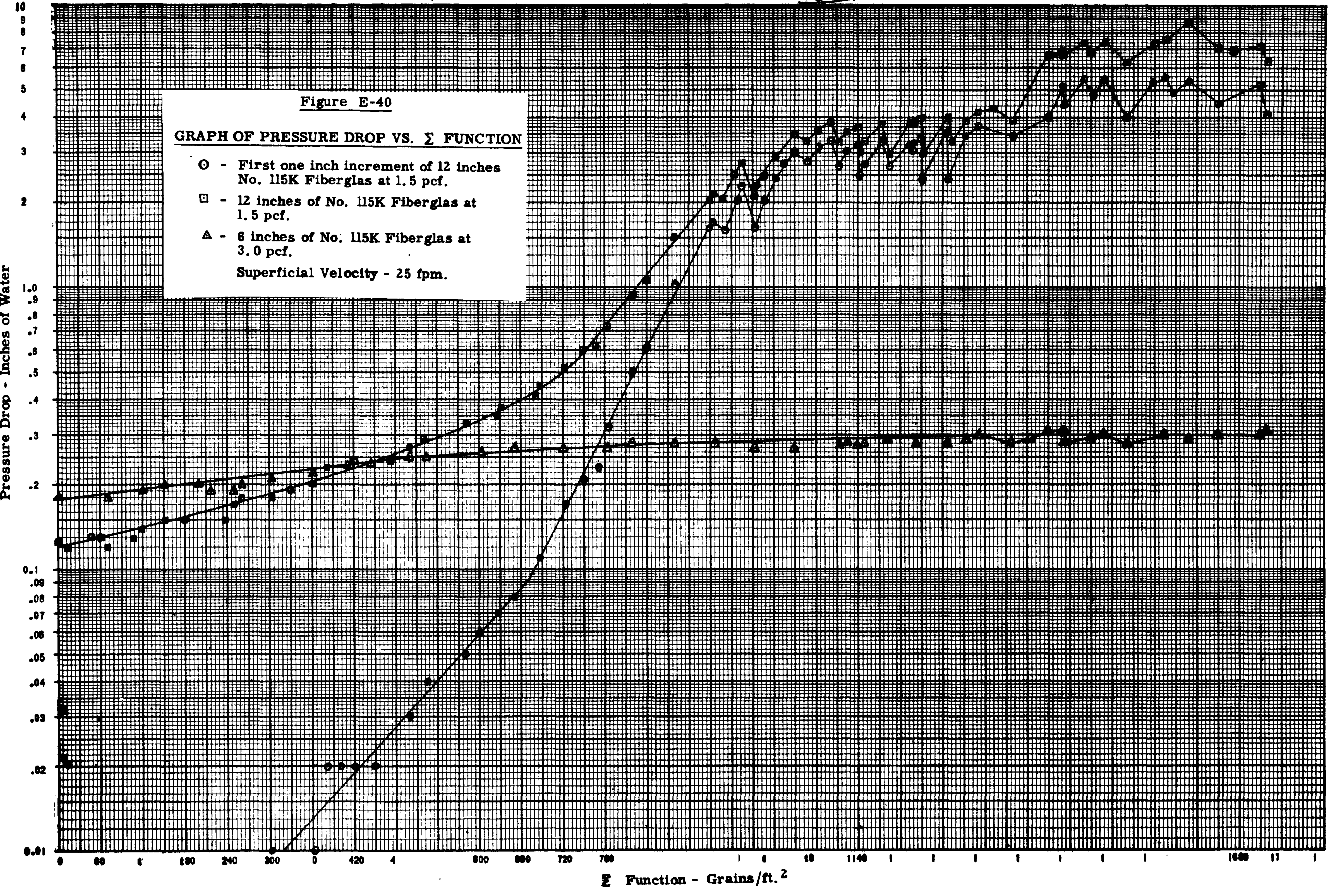

